\title{
A Coding of Social Class for the General Social Survey *
}

\author{
Stephen L. Morgan \\ Johns Hopkins University
}

GSS Methodological Report No. 125

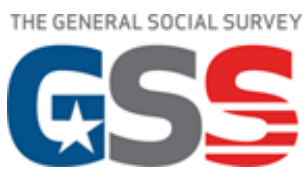

August 2017

* I thank Minhyoung Kang and Jiwon Lee for their research assistance, as well as Tom Smith and Jeremy Freese for their comments and suggestions. 


\section{Tables of Contents}

1. Introduction: Jobs, Occupations, and Social Class in the GSS $\ldots \ldots \ldots \ldots \ldots \ldots \ldots \ldots$

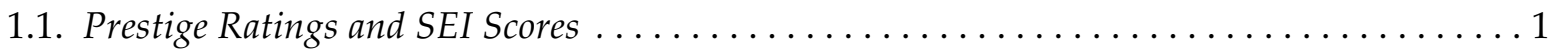

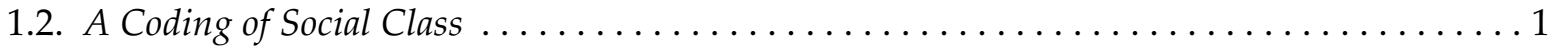

2. The Rationale and Strategy for Coding Social Class $\ldots \ldots \ldots \ldots \ldots \ldots \ldots \ldots \ldots$

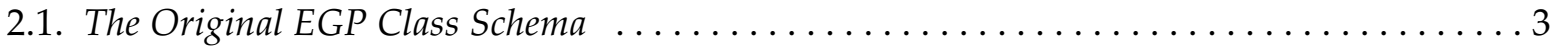

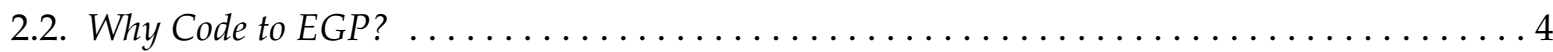

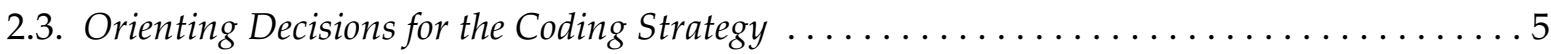

2.4. A Coding Strategy Based Only on the ACS, SOC, and ONET ................. 7

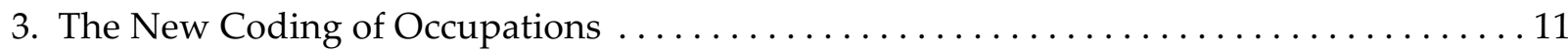

3.1. Classes IIIa and V: A Natural Evolution of the Original EGP Classes? .............. 11

3.2. New Class Descriptions with Illustrative Occupations . . . . . . . . . . . . . . . 12

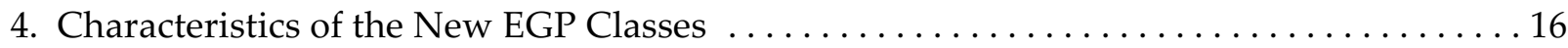

4.1. Characteristics of the Classes, Based on the ACS and ONET ................ 16

4.2. A Comparison with SOC Major and Minor Groups of Occupations . . . ........... 21

4.3. Characteristics of the Classes in the GSS and a (Plausibly) Aligned ACS Sample ........25

5. Sample Stata Code for Merging the .csv File and the GSS Cumulative File ... . . . . . . 37

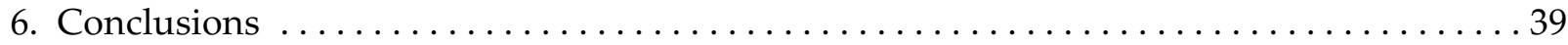

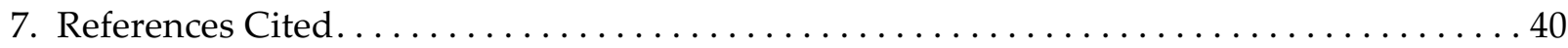

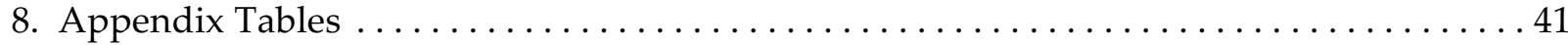

A1: GSS Occupational Distributions for the 10-Class Version of EGP . . . . . . . . 41

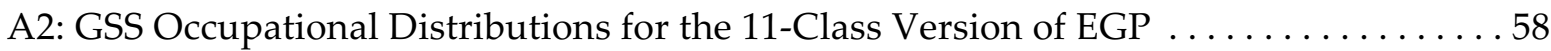

A3: GSS Occupational Distributions for the 12-Class Version of EGP $\ldots \ldots \ldots \ldots \ldots$. 


\section{Introduction: Jobs, Occupations, and Social Class in the GSS}

Beginning with the 2012 General Social Survey, job titles and job activity descriptions have been coded using only the 539-category 2010 US Census occupational classification (hereafter, "2010 census occupation codes"). As of July 2016, most job titles and descriptions from prior administrations of the GSS were also recoded to the 2010 census occupation codes. ${ }^{1}$ A reasonable expectation is that the 2010 census occupation codes will continue to be used for the GSS at least until 2022.

\subsection{Prestige Ratings and SEI Scores}

The 2010 census occupation codes can be used in many ways to model individual variation in other outcomes measured for the GSS. With the updated prestige ratings also developed during the fielding of the 2012 GSS (see Smith and Son 2014), two possibilities are to use either the new prestige scale (prestig10) or its associated SEI scale (sei10). These scales are now available for all individuals with 2010 census occupation codes, and their construction is explained in Hout, Smith, and Marsden (2016). For analysts who want a unidimensional scale of occupational standing, the SEI scores for the occupations are a very good choice. ${ }^{2}$

\subsection{A Coding of Social Class}

For analysts who wish to have a measure of social class, any principled coding of the 2010 occupation codes can be used to group related occupations into larger agglomerations, perhaps using additional information on individuals drawn from work histories and current labor market status. In the remainder of this report, I share the results of one such coding effort that I undertook in preparation for analysis of the 2016 GSS, during a time when conjectures about class-specific political attitudes and voting trends had received renewed attention in the United States.

\footnotetext{
${ }^{1}$ As of its summer 2016 release, the GSS cumulative data file includes multiple occupation codes for all years until and including 2010. The variable occ is present from 1972 through 1990 and is based on the 1970 census occupations codes. The variable occ 80 is present from 1988 through 2010 and is based on 1980 census occupations codes. However, from 2012 onward, only the 2010 codes are used for the new variable occ10.

2 As described in Hout et al. (2016), the values of the scale are near equal averages of threshold scales for educational attainment and personal earnings from a national sample of occupational incumbents, calculated using the public use microdata files of the American Community Survey (ACS). The near equal weighting of the scales for education and earnings for each occupation is justified by an analysis of the prestige ratings from GSS respondents' assessments of hundreds of job titles (see Smith and Son 2014). These ratings proved once again to be consistent with a long tradition of measurement of occupational prestige, and thus the variable sei10 is a direct analog to past SEI variables in the GSS (i.e., the sei variable available for 1988 through 2010).
} 
The specific social class coding explained in this report is available as a .csv file, occ10to-egp-class-crosswalk.csv, that is posted on the GSS website and my own personal website. The coding is also shown below in tabular form, as implemented for the 1994 GSS through 2016 GSS in Appendix Tables A1, A2, and A3 (beginning below on page 41).

Because this coding represents only one coding for how social class categories can be formed, it is not included as an official variable in any of the GSS data files. However, it can be matched to all GSS respondents who have valid codes for the variable occ10, as I demonstrate below with sample Stata code. The social class coding can also be used with minor modifications in any other data source that has occupation codes traceable to the 2010 Standard Occupational Classification of the United States, since this classification is the guiding framework for all occupational coding decisions in the United States since 2010. 


\section{The Rationale and Strategy for Coding Social Class}

\subsection{The Original EGP Class Schema}

Although many social class categorizations exist, I chose to code occupations to what I regard as the dominant schema in the international sociological literature on class differences. I will refer to this coding as the EGP class schema, which was debuted by Erikson, Goldthorpe, Portocarero $(1979,1982,1983)$ but is also sometimes referred to as Goldthorpe's class schema (after Goldthorpe 1987) or the EG class schema (after Erikson and Goldthorpe 1992). Its most prominent usage was in The Constant Flux, a broadly influential 1992 cross-national study of social mobility by Erikson and Goldthorpe.

Erikson and Goldthorpe (1992:41-42) describe each of their EGP classes in this way:

Class I

Higher-grade professionals, administrators, and officials; managers in large industrial establishments; large proprietors

\section{Class II}

Lower-grade professionals, administrators, and officials; higher-grade technicians; managers in small industrial establishments; supervisors of non-manual employees

\section{Class IIIa}

Routine non-manual employees, higher-grade (administration and commerce)

\section{Class IIIb}

Routine non-manual employees, lower-grade (sales and service)

\section{Class IVa}

Small proprietors, artisans, etc., with employees

Class IVb

Small proprietors, artisans, etc., without employees

\section{Class IVc}

Farmers and small-holders; other self-employed workers in primary production

\section{Class V}

Lower-grade technicians; supervisors of manual workers

\section{Class VI}

Skilled manual workers

\section{Class VIIa}

Semi- and unskilled manual workers (not in agriculture)

\section{Class VIIb}

Agricultural and other workers in primary production

As explained by Erikson and Goldthorpe, classes I, II, IIIa, and IIIb represent nonmanual workers, sorted in a principled hierarchy. Classes V, VI, and VIIa represent manual workers, also sorted in a principled hierarchy. Classes IVa and IVb represent self-employed workers, not in classes I and II and not in agriculture or primary production. These workers are sometimes discussed by Erikson and Goldthorpe as representing the classic "petty bourgeoisie," with class IVa differing from class IVb based on the scale of the self-employed 
worker's enterprise (and measured, when possible, by whether the individual has employees and, if so, how many). Class IVc represents farmers and others in primary production, usually self-employed, and class VIIb represents unskilled agricultural laborers and other similar workers, often those who are employed by members of class IVc. In studies of labor market outcomes, classes IVa, IVb, IVc, and VIIb are often dropped from analysis because of the divergent nature of their remuneration and work contexts.

Incumbents of classes I and II have employment contracts that typically have what is referred to in this tradition of analysis as a salary structure (and hence, classes I and II, are often labeled as composing the "salariat"). These individuals have skills that can be very specific to their occupations, and the monitoring of their daily performance by supervisors is difficult. They are therefore contracted as salaried employees, and their hourly and daily performance is not regularly monitored. In contrast, classes IIIb, VI, VIIa, and VIIb have employment contracts that approach, to varying degrees, a classic "spot contract," even if for transaction cost reasons or regulatory reasons they may not be paid on a piece rate or an hourly basis. Workers in these classes have skills that are less specific to their occupations, and their performance could be monitored daily, or even hourly, by supervisors, even if in practice this is not the case. Classes IIIa and V have employment contracts that are "mixed," with occupation and worker-specific variation, and with employment contracts with some blending of the features of both the salary and spot contract structures.

\subsection{Why Code to EGP?}

Although I embrace the core rationale of the EGP class schema, others eschew it. The primary reasons that I regard EGP classes as valuable and worthy of usage are

1. EGP is not unidimensional, even though hierarchy is embedded within it. For example, while class I exceeds class II on many indicators, no clear ranking exists for other classes, such as for classes V or VI relative to class IIIa, or for class IIIb relative to class VIIa. As such, EGP is a complementary categorization to unidimensional orderings. It can be used alongside other scales of occupations, such as those based on prestige ratings or simply the average education or earnings of occupational incumbents.

2. EGP has a tractable number of class categories, and it can be collapsed further (e.g., by combining classes I and II, classes V and VI, and classes IIIb and VIIa). This flexibility allows for parsimonious modeling, crisp characterizations, and continued usage in data sources that are of modest size (as is the case for each GSS crosssection).

3. EGP can represent the evolving post-industrial occupational structure. For example, the pattern of growth of differentially skilled "service sector" occupations is captured by the relative growth of classes I, II, and IIIa. The "working class" is 
usefully separated into a service-oriented class, IIIb, as well as two manual classes differing in typically required skills, classes VI and VIIa. In addition, the differences between these classes, as shown below, reveal the underlying sex segregation of occupations that are differentially distributed across classes.

These reasons notwithstanding, principled reasons exist to avoid the EGP class schema. The reasonable arguments of others are

1. Some researchers prefer class categories defined in opposition to each other. Class differences can then be characterized as the result of between-class contestation, usually resulting in asymmetrical exploitation. From this perspective, reliance on classes that represent agglomerations of occupational titles defined by government bureaucrats is a profane form of social science. For this argument, see Wright (1997).

2. Some researchers prefer class schemas with a larger number of categories (typically about 100, and often labeled "microclasses"). These more elaborate categorizations facilitate fine-grained analysis of job inheritance and the consequences of the values, cultures, and local institutions that are tightly bundled with some occupations, or small agglomerations thereof. For this argument, see Grusky and Sørensen (1998) and Weeden and Grusky (2005).

3. Some researchers prefer class categories, if any at all, to be based on patterns of consumption or post-materialist values, rather than the vestiges of working conditions that were characteristic of the mid-twentieth century industrial order. When class categories are organized primarily by market position and the acquisition of resources, they are claimed to be insufficiently predictive of behavior in the post-industrial age. For this argument, see Kingston (2000) and Pakulski and Waters (1996).

4. Some researchers (e.g., perhaps most labor economists) see little utility in the usage of class categorizations and argue instead for direct attention to individuals' "premarket" characteristics (e.g., years of completed education) and current labor market outcomes (e.g., wages earned). From this perspective, measures of social class are of relatively minor importance, even though effects attributable to particular occupations may be worthy of examination (e.g., to consider whether occupational licenses have meaningful effects above and beyond what can be predicted from individual-level measures).

\subsection{Orienting Decisions for the Coding Strategy}

My goal was to create a coding for occupations that would yield social classes in the spirit of the original EGP class schema, with full recognition that the distribution of job titles evolves yearly and that the original EGP class schema was a product of its foundational time 
period (the late 1970s through the early 1990s). ${ }^{3}$ Accordingly, several crucial decisions had to be taken at the outset.

First, I decided to code the 478 occupations that compose the 2012 occupational classification of the American Community Survey (hereafter, "ACS occupation codes"), which is expected to be used by the US Census Bureau for the ACS through 2020. All 539 census occupation codes that the GSS used for its own coding are embedded uniquely within each of the 478 categories of the ACS occupational classification. Thus, even though I coded the 478 occupations, the codes still allocate all 539 census occupations used for the GSS. I decided to code in this way because very little information from external sources on occupational incumbents is available to aid in coding the additional 2010 census occupations (i.e., the $479^{\text {th }}$ through $539^{\text {th }}$ occupations), given that the ACS replaced the US Census long form. I therefore had too little information to code the additional occupations with the same degree of care. In addition, coding the 478 occupations seemed forward-oriented, given the likelihood that many of the additional census occupations are small and thus candidates for being folded into larger categories in future occupational coding schemes promulgated by the US federal statistical agencies that collect occupation-related data. ${ }^{4}$

Unfortunately, this decision requires working with some awkward and clearly internally heterogeneous occupations in some cases (and which mistakenly imply, because of their labels, that the "folded in" occupation is as common as the larger "receiving" occupation). For example, the two census occupations of "Chief Executives" and "Legislators" are combined for the ACS as "Chief Executives and Legislators." It is not obvious to anyone that the job titles beneath "Chief Executives" and "Legislators" belong together in an occupational group. However, very little information is collected separately for legislators, either for prior microdata released to the public by the US Census Bureau or for the auxiliary ONET information described below that I used for coding. In other cases, the groupings are entirely reasonable, such as the combination of "Food preparation and serving related workers, all other" with "Dining room and cafeteria attendants and bartender helpers" which are combined for the omnibus ACS category of "Miscellaneous food preparation and serving related workers, including dining room and cafeteria attendants and bartender helpers." The occupational label is ungainly, but a consideration of the job titles listed in the SOC direct match file (described below) indicates that combinations such as these are sensible.

Second, I did not attempt to make the new EGP class coding of the ACS occupations consistent on an occupation-by-occupation basis with any prior efforts to code EGP classes using 1980 and 1990 US census occupation codes, including my own past work (e.g., see the Supplementary Appendices to Morgan and McKerrow 2004 and Morgan and Tang 2007). After

\footnotetext{
${ }^{3}$ In addition, Erikson and Goldthorpe indicated that their initial attempts to code the EGP class schema for the United States were unsuccessful (see Erikson and Goldthorpe 1985). There is, therefore, no urtext of the EGP class schema for the United States, only subsequent efforts.

${ }^{4}$ As Table A1 (below) shows for the GSS, these additional occupations are indeed very small in comparison to the 478 ACS occupations.
} 
initial exploration, I came to the position that the shift in census occupation codes that was debuted for 2000 (and carried forward to 2010) created very substantial inconsistency across occupations that seem similar based on their occupation labels (and this is consistent with a study conducted by the US Census Bureau; see Scopp 2003). ${ }^{5}$ Only for deeply institutionalized occupations was I confident that the occupation labels mapped to clearly defined and stable collections of underlying job titles. I decided to code the EGP classes without attempting to create similarities with prior coding decisions, even though, of course, a great many equivalences did emerge.

Third, I decided to code occupations into EGP classes in a first step that would ignore classes IVa and IVb. Although these two classes were sometimes labeled the "petty bourgeoisie" in early work with the EGP, they have come to be recognized in most subsequent empirical work in less grand terms: the self-employed members of occupations associated with classes IIIa, IIIb, V, VI, and VIIa. A final coding, which moves incumbents of the occupations allocated to classes IIIa, IIIb, V, VI, and VIIa into classes IVa and IVb can be implemented using self-employment variables in the GSS. ${ }^{6}$

Fourth, I decided that the four heterogeneous military occupations would be categorized separately as their own EGP "class." These are "Military officer special and tactical operations leaders," "First-line enlisted military supervisors," "Military enlisted tactical operations and air/weapons specialists and crew members, and "Military, rank not specified." For a large array of outcomes of interest, it is sensible to separate members of the military from the civilian labor force, even though the military "class" includes substantial heterogeneity of job duties, educational training, and work experiences.

\subsection{A Coding Strategy Based Only on the ACS, SOC, and ONET}

To inform coding decisions for the 478 ACS occupations, I assembled occupationspecific information from three primary sources:

1. The 2012-2015 public use microsamples of the ACS, which include individual-level data on the demographic characteristics, education, labor force status, and earnings of millions of occupational incumbents spread across all 478 occupations

2. Documentation for the 2010 Standard Occupational Classification (hereafter, "SOC"), including the textual SOC Definitions, which are paragraph descriptions of associated job duties, as well as the Direct Match Title File that associates job titles with each SOC occupation)

\footnotetext{
${ }^{5}$ I thank Minhyoung Kang for his research assistance in the initial coding, which was crucial in the way that it informed this later decision.

${ }^{6}$ If measures are available to determine whether self-employed workers have their own employees, and how many, these measures can be used to separate a combined class IVab into classes IVa and IVb. For the GSS, this separation is possible for 2004 onward.
} 
3. The ONET 21.0 Database, which can be matched to most SOC occupations, and which includes occupational ratings generated on a recurring survey of human resources professionals

Notice that the GSS itself is not listed above. The GSS data were not used to develop the coding of social class because the yearly samples are too small to yield reliable measures of the incumbents of all 478 occupations.

Aided by appropriate crosswalks that summarized the nesting of jobs and occupations, it was possible to construct a single file for all 478 occupations with information appropriately calculated from these sources (except the textual SOC definitions). In nearly all cases, some compromises were made in the construction of this file, such as when calculating averages across jobs/occupations to collapse the SOC-based ONET information to the ACS occupations (which, ultimately, was possible for only 463 of the 478 occupations).

With these sources of information in hand, I coded the occupations iteratively using my own judgment. In many cases, my initial decisions were superseded by new decisions after careful consideration of the ACS information on occupational incumbents, the SOC definitions and associated job titles contained in the SOC direct match job title file, the ONET information on the ratings of occupations, and the overall "employment relations" rationale of the EGP. I stopped coding when I was convinced that I could not improve the categorization any further without making decisions that were no better than prior decisions.

Although many of my decisions are consistent with EGP coding decisions based on 1980 and 1990 US census codes, some occupation-by-occupation comparisons of occupation labels will suggest differences. Some of these apparent differences reflect the SOC itself, which is substantially more detailed for many types of white-collar work. Many inconsistencies reflect better decisions enacted for this coding, in my view, that result from (1) improvements in the US Census Bureau's rules for assigning job titles and activities to occupations, as guided by the rationale underneath the SOC and (2) the new information available in the ONET and ACS that can be used to code resulting occupations into classes. ${ }^{7}$

7 See Scopp (2003; Table 2) for the results of a double-coding exercise that demonstrates the consequences of new coding rules for job titles and job activities first implemented for the 2000 US census. These new rules generated many differences in the underlying jobs associated with seemingly similar occupations. For this reason alone, some reallocation of occupations would be expected for any comparison of EGP classes based on 1980/1990 US census codes to EGP classes based on 2000/2010 US census codes. My sense is that the 2000/2010 coding rules are far better, both as occupation codes themselves and as can be used for the coding of EGP, especially for occupations that are clearly overbroad. Two examples are helpful:

1. The 1990 occupation 37, "Management related occupations, n.e.c.," was placed in class I for Morgan and Tang (2007) based on the EGP literature on the placement of managers. With the 2000 census occupations codes, as shown by Scopp (2003, Table 2), this group would be divided into five 
Regardless of comparisons to prior instantiations of the EGP class schema, some decisions will appear puzzling on their own, based only on consulting the occupation labels in Tables A1-A3 (and the associated .csv file). To some extent, such reactions are appropriate, since the fullness of a 478-part occupational categorization cannot be preserved for a coding of a dozen or fewer EGP classes. Small but distinct occupations, such as "Actors," "Animal Trainers," and "Athletes, Coaches, Umpires, and Related Workers," need to be placed somewhere.

In addition, many of the occupations are agglomerations of job titles that are not well described by their associated occupation labels. For example, the occupation "Other Teachers and Instructors" makes little sense on its own without a full consideration of the available information. The ACS revealed that the 2012 through 2015 incumbents of "Other Teachers and Instructors" had, on average, 15.2 years of education and personal earnings each year of $\$ 31,700$. When restricted to full-time, full-year workers, these incumbents had 15.1 years of education and $\$ 51,500$ in personal earnings. What these incumbents did in their jobs only became clear when the underlying job titles in the SOC direct match title file was consulted. The file offers illustrative job titles as "Tutor, Horseback Riding Instructor, Citizenship Teacher, Adult Education Teacher, Adult Literacy Instructor, General Educational Development (GED) Teacher, Substitute Teacher, and Sailing Instructor." It also lists additional non-illustrative job titles as "Ballet Teacher, Judo Instructor, Dance Teacher, Defensive Driving Instructor, CPR Instructor, Driver Education Instructor, Adult Basic Studies Teacher, Adult Remedial Education Instructor, Martial Arts Instructor, and Theater Education Teacher." After considering the full set of information available, I decided to place this occupation in class IIIa, rather than class I (where postsecondary teachers are placed) or class II (where elementary, middle, and secondary school teachers are placed). This decision is a good example of a tough coding choice, and in

occupations for the 2000 occupation codes: Cost estimators (21.6\%), Meeting and convention planners $(0.7 \%)$, Other business operations specialists (45.3\%), First-line supervisors of office and administrative support workers (6.5\%), and Secretaries and administrative assistants (25.9\%). For the new EGP coding described in this report, these five occupations are allocated across three separate classes (as "Business Operations Specialists, All Other" in class II, "Meeting, Convention, and Event Planners" in class IIIa, "Secretaries and Administrative Assistants" in class IIIa, "First-Line Supervisors of Office And Administrative Support Workers" in class IIIa, and "Cost Estimators" in class V.)

2. The 1990 occupation 5, "Administrators and officials, public administration," was placed in class I for Morgan and Tang (2007), based on the EGP literature on the placement of officials in large establishments. With the 2000 census occupation codes, as shown by Scopp (2003, Table 2), this same group of jobs would be allocated across 29 separate occupations, typically spread across both classes I and II. On the one hand, the largest of these 29 occupations is the 2000 census occupation of "Managers, All Other," which is folded into the 2012 ACS category of "Miscellaneous Managers, Including Funeral Service Managers and Postmasters and Mail Superintendents" (code 430, and where it is reasonable to assume "Miscellaneous Managers" dominate). This occupation is placed in the new EGP coding in class II, based on the criteria laid out in this report. On the other hand, the 29 occupations include some - such as "Chief Executives," "Financial Analysts," and "Judges, Magistrates, and Other Judicial Workers" - that are placed in the new EGP coding in class I. 
fact it was the final coding decision that I made. Class II might have been more appropriate if the average education and earnings of incumbents, as well as the ONET job zone rating, had been higher. In the end, I decided, based on the ACS results, that many individuals in this occupation had the profile of tutors and horseback riding instructors without bachelor's degrees, rather than secondary school teachers with bachelor's degrees who were temporarily employed as substitute teachers. 


\section{The New Coding of Occupations}

The original EGP class schema was developed during the twilight of the post-WorldWar-II industrial economy and while the information technology fueled post-industrial economy was in formation. The goal of my coding effort was to realize the benefits of the core rationale of the original EGP coding in a new coding appropriate for the analysis of today's stratification order (and a coding that can be used, with all due care, to reach back and analyze GSS data collected as early as 1972). I did not strive to replicate the fine features of the original EGP class schema, which was based on the occupational structure that prevailed several decades ago. Even if this were my goal, it would be impossible to succeed because of mismatches, detailed in the last section, in how jobs are assigned to the evolving structure of occupations from the year 2000 onward.

Having completed the coding, it is possible to now ask the question: Is this new coding still properly referred to as EGP? The ultimate answer is in the eye of the beholder. My position is that this coding is EGP for our time, tailored to the labor market of the United States.

Before presenting the characteristics of the classes generated by this coding, in the next section I consider classes IIIa and V. These are the classes where it could be argued that I have introduced some implicit but modest updating for this EGP coding in comparison to its predecessors. It is notable, however, that these are the classes that Goldthorpe and his colleagues have long recognized have "mixed" types of employment contracts. It is here, therefore, that one might expect change to emerge more prominently. And, if so, no updating has occurred in my construction of the schema, and these classes only appear slightly updated because of underlying shifts in the division of labor, and its contracting, in the post-industrial United States.

\subsection{Classes IIIa and V: A Natural Evolution of the Original EGP Classes?}

For this coding, some occupations that the extant EGP literature suggests could be placed in class II were instead allocated to class IIIa or class V based on their characteristics, seen in light of the structure of the current division of labor. The occupational structure has evolved in the last three decades with substantial skill upgrading for many occupations, along with a blurring of the boundaries in the employment relations between occupations at the lower end of what Erikson and Goldthorpe labeled the "salariat" (i.e., classes I and II) and those at the higher-end of class IIIa, which they labeled "routine non-manual employees, higher-grade (administration and commerce)." The occupation of "Human Resource Workers" is a good example of a class IIIa occupation that ranks lower on all dimensions than the class II occupation of "Human Resource Managers," but not so much lower that the traditional EGP label of "routine non-manual employee" feels quite right anymore. Accordingly, the common practice, when analyzing datasets of modest size, of combining the original EGP classes I and II into a salariat class and classes IIIa and IIIb into a routine non-manual service class seems less 
appropriate for this new coding. Classes I, II, IIIa, and IIIb appear in the new coding in a clear ordering, with a break between II and IIIa a bit less apparent now than in the past.

Relatedly, class $\mathrm{V}$ in the new coding is even more heterogeneous than users of past versions of EGP might expect. This result, however, is consistent with past analysis practices, in which class $\mathrm{V}$ is treated as being the most heterogeneous in its associated employment relations. From this perspective, class $\mathrm{V}$ has always been a "middling" class for occupations that confer advantages on incumbents relative to those in classes IIIb, VI, and VIIa, are more physical or technical than many of the cubicle-based occupations typical of class IIIa, and that clearly do not fit within classes I or II because of either lower levels of education or earnings (and whose inclusion in classes I or II would compromise the boundaries of classes I and II). Public safety workers (police officers and firefighters) are a prime example. More awkward are the small, but hard to categorize, occupations for performers ("Actors" and "Announcers," etc.) whose positions, when measured objectively by their incumbents' characteristics, and ONET ratings, are not as high-minded as one might otherwise assume from lay portrayals.

\subsection{New Class Descriptions with Illustrative Occupations}

With recognition of how the EGP classes have evolved slightly in the post-industrial economy of the United States, the following updated class descriptions are more accurate for this coding than prior EGP class descriptions. I also offer illustrative occupations below.

\section{Class I}

Higher-grade professionals, administrators, managers, and officials

Illustrative occupations:

Accountants and Auditors

Chief Executives and Legislators

Civil Engineers

Computer and Information Systems Managers

Electrical and Electronics Engineers

Lawyers, and Judges, Magistrates, And Other Judicial Workers

Management Analysts

Mechanical Engineers

Medical and Health Services Managers

Pharmacists

Physicians and Surgeons

Postsecondary Teachers

Software Developers, Applications and Systems Software

\section{Class II}

Lower-grade professionals, administrators, managers, and officials

Illustrative occupations:

Clergy 
Computer Programmers

Computer Systems Analysts

Counselors

Elementary and Middle School Teachers

Financial Managers

General and Operations Managers

Human Resources Managers

Marketing and Sales Managers

Secondary School Teachers

Social Workers

Registered Nurses

\section{Class IIIa}

Routine non-manual and service employees, higher-grade

Illustrative occupations:

Bookkeeping, Accounting, and Auditing Clerks

Computer Support Specialists

Customer Service Representatives

First-Line Supervisors of Office and Administrative Support Workers

Human Resources Workers

Licensed Practical and Licensed Vocational Nurses

Office Clerks, General

Other Teachers and Instructors

Real Estate Brokers and Sales Agents

Sales Representatives, Services, All Other

Sales Representatives, Wholesale and Manufacturing

Secretaries and Administrative Assistants

Teacher Assistants

\section{Class IIIb}

Routine non-manual and service employees, lower-grade

Illustrative occupations:

Cashiers

Childcare Workers

Combined Food Preparation and Serving Workers, Including Fast Food

First-Line Supervisors of Retail Sales Workers

Hairdressers, Hairstylists, and Cosmetologists

Nursing, Psychiatric, and Home Health Aides

Personal Care Aides

Receptionists and Information Clerks

Retail Salespersons

Security Guards and Gaming Surveillance Officers

Waiters and Waitresses

\section{Class IVa}

Non-professional self-employed workers with employees 


\section{Class IVb}

Non-professional self-employed workers without employees

\section{Class IVc}

Owners and managers of agricultural establishments

Illustrative occupations:

Farmers, Ranchers, and Other Agricultural Managers

\section{Class V}

Higher-grade technicians and repairers, public safety workers, performers, and supervisors of manual workers

Illustrative occupations:

Bailiffs, Correctional Officers, And Jailers

Chefs and Head Cooks

Clinical Laboratory Technologists and Technicians

Construction Managers

Drafters

Diagnostic Related Technologists and Technicians

Engineering Technicians, Except Drafters

Firefighters

First-Line Supervisors of Construction Trades and Extraction Workers

First-Line Supervisors of Mechanics, Installers, And Repairers

First-Line Supervisors of Non-Retail Sales Workers

First-Line Supervisors of Production and Operating Workers

Health Practitioner Support Technologists and Technicians

Police Officers

\section{Class VI}

Skilled manual workers, lower-grade technicians, installers, and repairers

Illustrative occupations:

Automotive Service Technicians and Mechanics

Brickmasons, Blockmasons, Stonemasons, and Reinforcing Iron and Rebar Workers

Bus and Truck Mechanics and Diesel Engine Specialists

Carpenters

Computer, Automated Teller, and Office Machine Repairers

Drywall Installers, Ceiling Tile Installers, and Tapers

Electrical Power-Line Installers and Repairers

Electricians

Heating, Air Conditioning, and Refrigeration Mechanics and Installers

Industrial and Refractory Machinery Mechanics

Machinists

Maintenance and Repair Workers, General

Painters and Paperhangers 
Pipelayers, Plumbers, Pipefitters, and Steamfitters

Sheet Metal Workers

\section{Class VIIa}

Semiskilled and unskilled manual workers, not in agriculture

Illustrative occupations:

Butchers and Other Meat, Poultry, and Fish Processing Workers

Construction Laborers

Cooks

Dishwashers

Driver/Sales Workers and Truck Drivers

Electrical, Electronics, and Electromechanical Assemblers

Food Preparation Workers

Grounds Maintenance Workers

Janitors and Building Cleaners

Laborers and Freight, Stock, And Material Movers, Hand

Laundry and Dry-Cleaning Workers

Maids and Housekeeping Cleaners

Packaging and Filling Machine Operators and Tenders

Packers and Packagers, Hand

Refuse and Recyclable Material Collectors

Roofers

\section{Class VIIb}

Agricultural workers and their first-line supervisors, and other workers in primary production

Illustrative occupations:

Miscellaneous Agricultural Workers, Including Animal Breeders

\section{Military}

All members of the armed forces

Illustrative occupations:

First-Line Enlisted Military Supervisors

Military Enlisted Tactical Operations and Air/Weapons Specialists and Crew Members

Military Officer Special and Tactical Operations Leaders

Military, Rank Not Specified 


\title{
4. Characteristics of the New EGP Classes
}

\author{
Important Note of Clarification
}

In this section, and subsequent sections, I present characteristics of three related EGP class categorizations.

The 10-class version of EGP considered first does not include classes IVa or IVb. It is based only on the coding of the 478 occupations in the ACS occupational categorization. In other words, self-employed individuals in classes IIIa, IIIb, V, VI, and VIIa have not been reallocated to classes IVa and IVb, and for this reason I often use the phrase "occupation only" EGP classes when referring to the 10-class version.

When information on self-employment is utilized (either for the ACS or the GSS), two additional EGP class categorizations can be formed: an 11-class version of EGP with one additional class (IVab) and a 12-class version with two additional classes (IVa and IVb as separate classes).

Because coding decisions were shaped most importantly by decisions for how to allocate the 478 occupations to classes I, II, IIIa, IIIb, IVc, V, VI, VIIa, VIIb, and "Military," this section considers the 10-class version of EGP in considerable detail. The 11-class and 12-class versions are introduced later in this section, and analyzed in less detail.

\subsection{Characteristics of the Classes, Based on the ACS and ONET}

Table 1 presents class-specific means of the characteristics of occupational incumbents in the 2012-15 ACS. It also includes class-specific means of the GSS prestige and SEI values (see Hout et al. 2016) when attached to these ACS individuals. Table 2 presents class-specific means of the ONET scales that were used for coding. Although the ACS and ONET information presented in Tables 1 and 2 is reported at the EGP class level, when utilized for coding decisions it was consulted as occupation-specific means in the case of the ACS and as occupation-specific ratings in the case of the ONET (where these occupation-specific ratings were often averages of underlying ratings of more detailed SOC occupations).

Many of the coding decisions for each occupation were very close, and the information summarized in Tables 1 and 2 was used to adjudicate the close calls. The ONET was used less frequently than the ACS information, but it proved useful in many cases. As can be seen in Table 2, the coding resulted in ONET "service importance" and "service level" ratings that differentiate the service-oriented classes IIIa and IIIb from the manual classes VI, VIIa, and VIIb. Similarly, to help with close calls when differentiating occupations in classes I, II, and V from classes IIIa, IIIb, VI, VIIa, and VIIb, the ONET "decision freedom" and "unstructured work" ratings were utilized. And, as shown in Table 2, little difference is shown on the means for these ONET ratings between classes IIIa, IIIb, VI, and VIIa because all of the occupations in these classes are often similarly routine and structured. 
Table 1. Characteristics of Occupational Incumbents in the 2012-2015 ACS by EGP Class (10-class version)

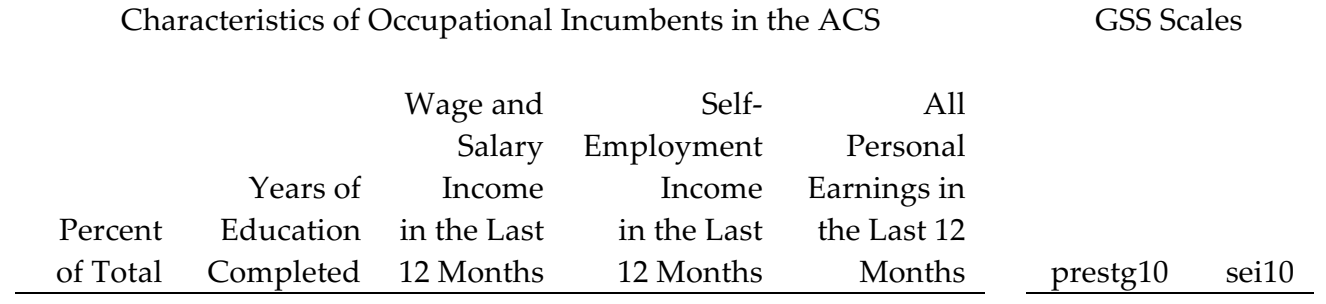

For full-time,

full-year

workers:

$\begin{array}{lrrrrrrr}\text { I } & 12.2 & 16.8 & 108,652 & 6,038 & 114,691 & 65.1 & 83.0 \\ \text { II } & 20.0 & 15.7 & 77,786 & 2,392 & 80,177 & 53.8 & 73.5 \\ \text { IIIa } & 18.1 & 14.0 & 52,372 & 2,047 & 54,419 & 42.7 & 47.4 \\ \text { IIIb } & 13.7 & 13.1 & 37,881 & 2,736 & 40,618 & 37.2 & 32.5 \\ \text { IVc } & 0.4 & 13.1 & 29,536 & 41,245 & 70,781 & 46.3 & 39.9 \\ \text { V } & 10.4 & 13.8 & 61,884 & 3,272 & 65,156 & 49.6 & 54.4 \\ \text { VI } & 7.1 & 12.3 & 46,391 & 3,409 & 49,800 & 42.6 & 36.7 \\ \text { VIIa } & 17.2 & 11.9 & 36,210 & 2,087 & 38,298 & 31.7 & 24.8 \\ \text { VIIb } & 0.5 & 11.0 & 30,613 & 3,431 & 34,044 & 30.2 & 18.3 \\ \text { Military } & 0.3 & 14.3 & 59,668 & 43 & 59,711 & 61.1 & 54.0\end{array}$

For all

workers:

\begin{tabular}{lrrrrrrr} 
I & 10.0 & 16.8 & 92,968 & 5,983 & 98,951 & 65.2 & 83.0 \\
II & 17.5 & 15.7 & 64,050 & 2,311 & 66,362 & 54.5 & 74.0 \\
IIIa & 17.8 & 13.9 & 38,779 & 1,814 & 40,593 & 42.5 & 46.1 \\
IIIb & 17.8 & 13.0 & 23,639 & 1,945 & 25,584 & 36.4 & 30.4 \\
IVc & 0.4 & 13.0 & 22,302 & 32,423 & 54,725 & 46.3 & 39.9 \\
V & 9.2 & 13.8 & 49,745 & 3,360 & 53,105 & 49.6 & 54.3 \\
VI & 6.7 & 12.2 & 35,917 & 3,353 & 39,270 & 42.3 & 35.6 \\
VIIa & 19.6 & 11.8 & 24,600 & 1,788 & 26,387 & 30.9 & 23.5 \\
VIIb & 0.7 & 10.8 & 19,747 & 2,334 & 22,081 & 29.5 & 17.4 \\
Military & 0.3 & 14.2 & 50,497 & 45 & 50,542 & 60.9 & 53.7 \\
\hline
\end{tabular}

Source: American Community Surveys, 2012 through 2015 PUMS Data Files (extracted from the 2011-2015 file) Notes: The 2012-15 ACS sample was restricted to respondents aged 25 to 64 not living in institutionalized group quarters. Across years, dollar values are inflation adjusted to 2015 dollars. "Full-time, full-year workers" are defined as (1) having worked at least 50 weeks, including vacation days, in the prior 12 months, (2) having usually worked 35 or more hours per week in the prior 12 months, and (3) having reported earnings of at least \$10,440 (in 2015 dollars) from the prior 12 months. The 2012-2015 ACS includes 3,429,382 individuals between the ages of 25 and 64 meeting this definition of full-time, full-year workers. When weighted, these individuals represent an average yearly adult full-time, full-year labor force of 90,263,709 individuals between the ages of 25 and 64. "All workers" are defined as all individuals with a valid occupation code, and occupation codes are not assigned to individuals who have never worked or who have not worked within the past five years. The 2012-2015 ACS includes 5,495,714 total sampled individuals of this type (and, when weighted, these individuals represent an average yearly adult worker labor force of 142,581,005 individuals between the ages of 25 and 64). 
Table 2. ONET Scales by EGP Class (10-class version), as Weighted Means of Occupation-Specific Values Across 478 Occupations

\begin{tabular}{|c|c|c|c|c|c|c|}
\hline Required & Related & & Service & Service & & \\
\hline Years of & Years of & Job & Orientation & Orientation & Decision & Unstructured \\
\hline Education & Experience & Zone & (Level) & (Importance) & Freedom & Work \\
\hline
\end{tabular}

For full-time,

full-year

workers:

$\begin{array}{lrrrrrrr}\text { I } & 17.2 & 4.3 & 4.5 & 3.2 & 3.1 & 4.3 & 4.3 \\ \text { II } & 15.8 & 3.4 & 3.9 & 3.5 & 3.4 & 4.3 & 4.3 \\ \text { IIIa } & 13.7 & 2.3 & 3.0 & 3.2 & 3.3 & 4.0 & 4.3 \\ \text { IIIb } & 12.4 & 1.2 & 2.1 & 3.2 & 3.5 & 4.0 & 4.0 \\ \text { IVc } & 14.2 & 3.9 & 3.7 & 2.9 & 2.9 & 4.6 & 4.5 \\ \text { V } & 13.9 & 3.4 & 3.1 & 3.1 & 3.1 & 4.3 & 4.1 \\ \text { VI } & 12.4 & 2.6 & 2.6 & 2.4 & 2.5 & 4.2 & 3.9 \\ \text { VIIa } & 11.8 & 0.9 & 1.9 & 2.2 & 2.5 & 3.8 & 3.6 \\ \text { VIIb } & 11.9 & 1.5 & 1.9 & -2.1 & 2.4 & 4.0 & 4.0 \\ \text { Military } & -- & -- & -- & -- & -- & -- & -\end{array}$

For all

workers:

\begin{tabular}{lrrrrrrr} 
I & 17.2 & 4.2 & 4.5 & 3.2 & 3.1 & 4.3 & 4.3 \\
II & 15.9 & 3.2 & 3.9 & 3.5 & 3.4 & 4.3 & 4.3 \\
IIIa & 13.7 & 2.2 & 3.0 & 3.2 & 3.3 & 4.0 & 4.2 \\
IIIb & 12.4 & 1.1 & 2.1 & 3.2 & 3.5 & 4.0 & 3.9 \\
IVc & 14.2 & 3.9 & 3.7 & 2.9 & 2.9 & 4.6 & 4.5 \\
V & 13.9 & 3.4 & 3.2 & 3.0 & 3.1 & 4.2 & 4.1 \\
VI & 12.4 & 2.6 & 2.6 & 2.4 & 2.6 & 4.2 & 3.9 \\
VIIa & 11.8 & 0.9 & 1.9 & 2.3 & 2.6 & 3.8 & 3.6 \\
VIIb & 11.8 & 1.4 & 1.8 & 2.0 & 2.4 & 4.0 & --9 \\
Military & -- & -- & -- & - & - & - \\
\hline
\end{tabular}

Source: ONET 21.0 (matched to the same individuals for Table 1, and tabulated as class-specific means)

Notes: Job Zone is a five-category scale for required preparation for the job, combining educational training, related experience, and on-the-job training, with 1 "Little or no preparation needed" to 5 "Extensive preparation needed." Service Orientation (Level and Importance) are indicators for the skills scale based on the analyst survey. The intent of the scale appears to be capture the orientation toward assisting people while on the job, rather than working with materials. Decision Freedom and Unstructured Work are indicators for the work context scale based on the analyst survey, from 1 "No Freedom" to 5 "A lot of Freedom." 
Table 3 presents additional ACS information on occupational incumbents, and this information was not considered when developing the coding. It was calculated afterwards, and I present the class differences because they demonstrate that other characteristics of occupational incumbents are associated with the EGP distinctions, even though they were not the basis of the coding. Most prominent, perhaps, is the sex segregation of the occupations summarized at the class level by the differences in percent female. In particular, classes II, IIIa, and IIIb are majority female, while all other classes are majority male. 
Table 3. Additional Characteristics of Occupational Incumbents in the 2012-2015 ACS by EGP Class (10-class version)

\begin{tabular}{rrrrrrrr} 
& Percent & & & & & \\
& White and & & Percent & Percent & Percent & Percent & Percent \\
Percent & Non- & Mean & Foreign & Private & Public & Self- & Full-time, \\
Female & Hispanic & Age & Born & Employer & Employer & Employed & Full-year \\
\hline
\end{tabular}

For full-

time, full-

year

workers:

\begin{tabular}{|c|c|c|c|c|c|c|c|c|}
\hline I & 38.0 & 73.9 & 44.2 & 19.0 & 71.6 & 18.7 & 9.7 & All \\
\hline II & 53.6 & 75.3 & 43.6 & 12.1 & 69.7 & 25.1 & 5.2 & All \\
\hline IIIa & 67.2 & 70.2 & 44.2 & 10.7 & 77.8 & 17.2 & 5.0 & All \\
\hline $\mathrm{IIIb}$ & 57.8 & 59.9 & 42.3 & 20.0 & 82.5 & 7.0 & 10.5 & All \\
\hline IVc & 10.0 & 91.4 & 47.3 & 7.0 & 23.2 & 0.7 & 76.0 & All \\
\hline V & 26.2 & 72.5 & 43.3 & 12.4 & 65.9 & 25.0 & 9.1 & All \\
\hline VI & 4.2 & 68.5 & 43.5 & 19.6 & 79.3 & 9.4 & 11.4 & All \\
\hline VIIa & 23.6 & 51.6 & 43.7 & 29.4 & 85.4 & 8.3 & 6.4 & All \\
\hline VIIb & 18.6 & 39.6 & 42.0 & 50.6 & 87.3 & 4.1 & 8.6 & All \\
\hline Military & 11.2 & 69.1 & 34.2 & 6.2 & 0.0 & 100.0 & 0.0 & All \\
\hline
\end{tabular}

For all

workers:

\begin{tabular}{|c|c|c|c|c|c|c|c|c|}
\hline I & 41.8 & 73.5 & 44.4 & 19.6 & 69.1 & 19.3 & 11.6 & 77.6 \\
\hline II & 58.4 & 75.6 & 44.2 & 12.1 & 66.5 & 26.8 & 6.7 & 72.1 \\
\hline IIIa & 71.4 & 70.3 & 44.4 & 11.3 & 76.1 & 17.0 & 6.9 & 64.3 \\
\hline $\mathrm{IIIl}$ & 67.2 & 60.8 & 42.2 & 19.0 & 81.4 & 7.1 & 11.5 & 48.7 \\
\hline IVc & 16.3 & 89.7 & 47.9 & 8.0 & 20.4 & 0.7 & 78.9 & 67.1 \\
\hline V & 30.8 & 72.5 & 43.7 & 13.0 & 64.9 & 21.8 & 13.3 & 72.0 \\
\hline VI & 5.2 & 68.2 & 44.1 & 19.5 & 76.5 & 7.7 & 15.9 & 66.6 \\
\hline VIIa & 30.3 & 51.9 & 43.5 & 27.8 & 83.5 & 7.2 & 9.3 & 55.6 \\
\hline VIIb & 30.7 & 36.0 & 42.3 & 52.7 & 86.9 & 2.8 & 10.3 & 47.5 \\
\hline Military & 12.3 & 67.7 & 34.5 & 6.5 & 0.0 & 100.0 & 0.0 & 79.4 \\
\hline
\end{tabular}

Source: See Table 1.

Notes: For the sample compositions for each panel, see the notes to Table 1. Percent white and non-Hispanic is the percent of respondents who indicate that they are non-Hispanic and are designated for race as having selected white alone or white in combination with another race. 


\subsection{A Comparison with SOC Major and Minor Groups of Occupations}

Table 4 offers an assessment of how the 10-class, occupation-only EGP, as well as an extended 11-class version with class IVab, fares in comparison to two other reduced representations of the 478 ACS occupations. One is the 23-category "major" grouping of the SOC:

$\begin{array}{ll}11 & \text { Management Occupations } \\ 13 & \text { Business and Financial Operations Occupations } \\ 15 & \text { Computer and Mathematical Occupations } \\ 17 & \text { Architecture and Engineering Occupations } \\ 19 & \text { Life, Physical, and Social Science Occupations } \\ 21 & \text { Community and Social Service Occupations } \\ 23 & \text { Legal Occupations } \\ 25 & \text { Education, Training, and Library Occupations } \\ 27 & \text { Arts, Design, Entertainment, Sports, and Media Occupations } \\ 29 & \text { Healthcare Practitioners and Technical Occupations } \\ 31 & \text { Healthcare Support Occupations } \\ 33 & \text { Protective Service Occupations } \\ 35 & \text { Food Preparation and Serving Related Occupations } \\ 37 & \text { Building and Grounds Cleaning and Maintenance Occupations } \\ 39 & \text { Personal Care and Service Occupations } \\ 41 & \text { Sales and Related Occupations } \\ 43 & \text { Office and Administrative Support Occupations } \\ 45 & \text { Farming, Fishing, and Forestry Occupations } \\ 47 & \text { Construction and Extraction Occupations } \\ 49 & \text { Installation, Maintenance, and Repair Occupations } \\ 51 & \text { Production Occupations } \\ 53 & \text { Transportation and Material Moving Occupations } \\ 55 & \text { Military Specific Occupations }\end{array}$

The other is the 98-category "minor" grouping of the SOC:

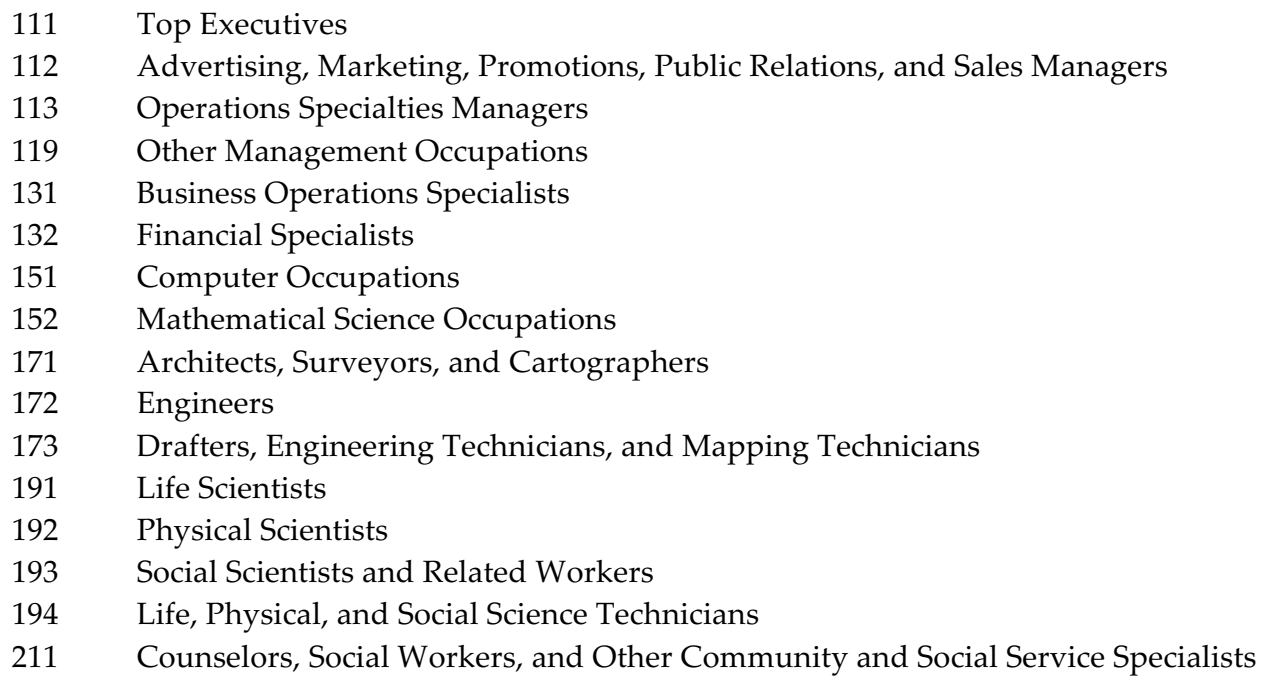


Religious Workers

231 Lawyers, Judges, and Related Workers 


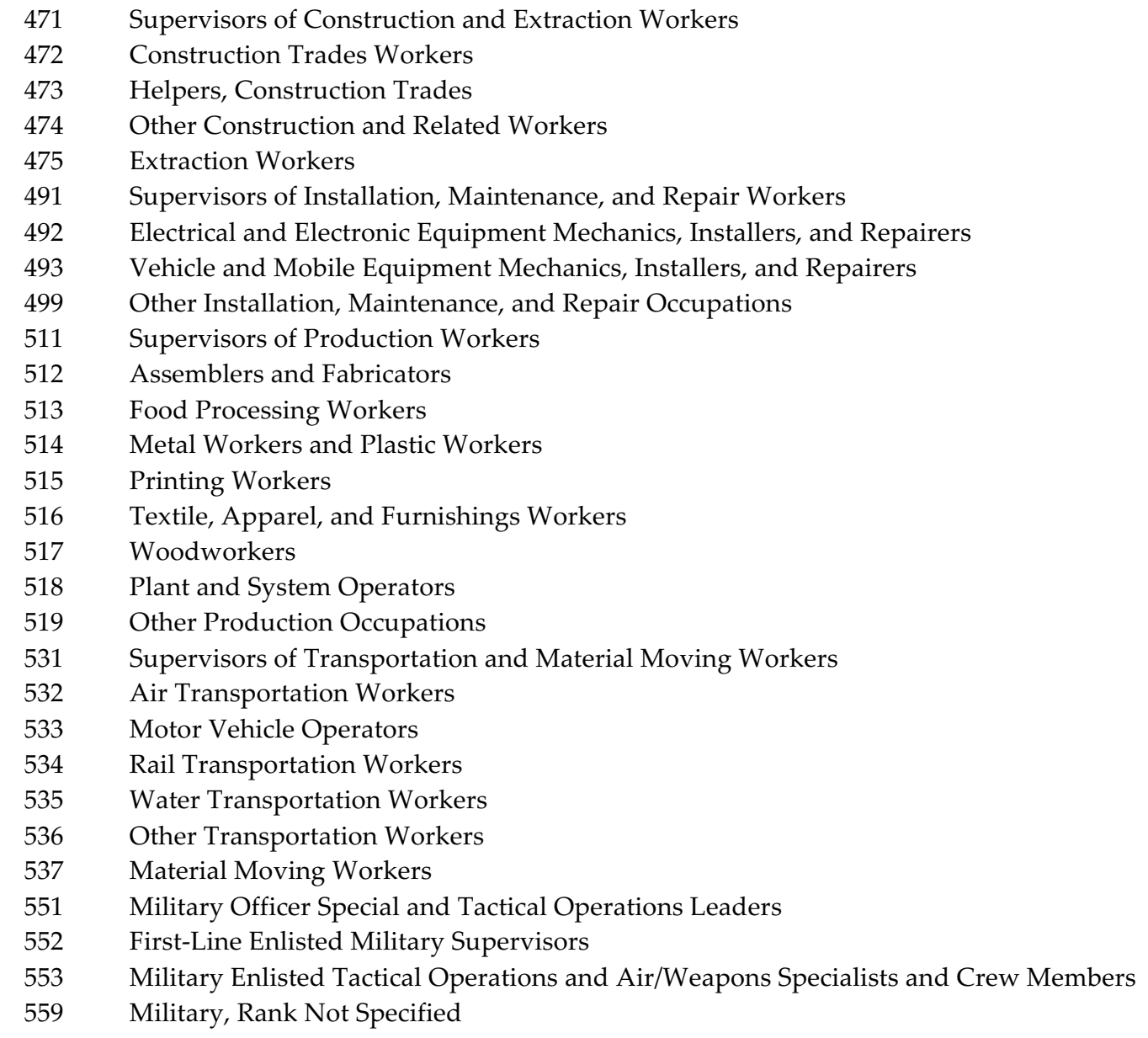

The 98 minor SOC groups are embedded in the 23 major SOC groups. EGP classes do not map directly to either of these categorizations, with nearly all SOC "major" and "minor" categories distributed across more than one EGP class, even though the number of EGP classes is much smaller.

Table 4 presents R-squared values for many different regression specifications for the prediction of the log earnings and years of completed education of ACS respondents, aged 2564 who have valid occupation codes. The R-squared values are interpretable as the proportion of the variance of the outcome variable listed in column label that be accounted for by the specification described in the row label. Each set of regression equations was estimated for two different samples of the ACS, and variables were typically given factor specifications (i.e., 9 or 10 indicator variables for the 10 or 11 EGP classes, and 10 indicator variables for the 11 groups of completed education). 
Table 4. R-squared Values for Least Squares Prediction of Log Earnings and Years of Education from Individual-Level ACS Data, Using Alternative Specifications for 10-class and 11-class versions of EGP

Log earnings

All worker with reported earnings
Years of Education

All adults

with a Full-time,

Full-time, full- reported full-year year workers occupation workers

Models

0.174

0.177

0.251

0.249

0.388

0.380

0.393

1B. EGP (11 classes, with IVab)

0.171

0.236

0.302

0.301

0.353

0.423

0.421

0.301

0.326

0.412

0.437

0.326

0.437

0.225

0.303

0.302

0.114

0.192

0.196

7B. Model $6+$ EGP (11 classes)

8. SOC minor (98 groups), completed education (11 groups), gender, race-ethnicity, and experience

9A. Model $8+$ EGP (10 classes)

9B. Model $8+$ EGP (11 classes)

10. SOC minor (98 groups), gender, race-ethnicity, and experience

11A. Model $10+$ EGP (10 classes)

11B. Model $10+$ EGP (11 classes)

$\begin{array}{ll}0.289 & 0.428 \\ 0.296 & 0.436 \\ 0.302 & 0.436\end{array}$

$-$

$-$
$-$

$-$
0.464

0.487

0.487
0.389

0.361

0.434

0.433

0.421

0.450

0.449

-- - -

Source: See Table 1.

Notes: For the sample compositions of the last three columns, see the notes to Table 1. The sample composition of the first column is the sample for the third column further restricted to those who reported positive earnings. 
Overall, the EGP categorization performs as expected, given how the coding decisions were made. Comparing Models $1 \mathrm{~A}$ and $1 \mathrm{~B}$ to Model 2, indicator variables for the 10-category and 11-category versions of EGP predict substantially more of each outcome variable than the 23-category SOC major groups. Comparing Models 1A and 1B to Model 6, EGP also predicts log earnings more strongly than the 11 groups defined by completed years of education. Comparing Model 1A and 1B to Model 4, EGP always predicts less of the variance than the 98category SOC minor groups, and more so for log earnings than for years of education. But EGP still fares well, especially with recognition of the fact that it has only 10 or 11 categories rather than 98 categories. Finally, comparisons within the five triples of models (e.g., Models 2, 3A, and $3 \mathrm{~B}$ ) show the incremental prediction gain from adding EGP to the variables for the first model in each triple. In all five cases, EGP classes deliver meaningful additional predictive variance.

Nonetheless, it should be kept in mind that the coding decisions themselves were made based to a substantial extent on the dependent variables in these models. Accordingly, it would be odd indeed if EGP did not effectively predict both log earnings and education. That EGP does better than the 23 major SOC groups, and is not too far from the 98 minor SOC groups, is nonetheless encouraging, at least in suggesting that substantial variation can be predicted by a categorization that has many fewer categories.

\subsection{Characteristics of the Classes in the GSS and a (Plausibly) Aligned ACS Sample}

How effective is the EGP coding for GSS respondents? The GSS collects information on a small fraction of the number of individuals sampled for the ACS, and so it is reasonable to wonder how well EGP performs in a dataset with typically no more than 3,000 respondents (and usually substantially fewer), many of whom are not in the labor force. The most natural way to assess the viability of the coding is to consider GSS-based classes alongside ACS-based classes. This section offers reassuring evidence that no new challenges arise when the coding is implemented for the GSS.

However, it should be stressed at the outset that no perfectly aligned analysis can be performed. The GSS is a national sample of English and Spanish speakers living in housing units (and prior to 2006, only English speakers). The ACS is a national sample of all individuals living in the United States. Although the ACS group quarters sample can easily be dropped to better align with the target population of the GSS, there is no obvious way to subset the ACS records to form a random sample of English and Spanish speakers. ${ }^{8}$

Question differences are an even greater obstacle to a perfectly aligned comparative analysis, given mode of response, wording, and reference periods for employment, work status,

\footnotetext{
${ }^{8}$ Other differences related to sampling and nonresponse exist as well. The ACS PUMS record selection, as well as weights, incorporate adjustments based on other census data sources. The GSS does not use these adjustments. The ACS imputes item-specific nonresponse using donor records. The GSS does not impute item-specific nonresponse.
} 
occupation, and earnings categories, both within and between the ACS and GSS. While race, ethnicity, gender, and place of birth are elicited with very similar questions, some crucial differences are present for others. To give a sense of these comparison challenges, here is some additional detail for how the ACS and GSS differ when eliciting occupations and earnings:

Occupation. For the ACS, the occupation elicited is the one held at the time of the survey or "most recently." The 2015 instrument instructs that the person completing the form "Describe clearly this person's chief job activity or business last week. If this person had more than one job, describe the one at which this person worked the most hours. If this person had no job or business last week, give information for his/her last job or business." Respondents are then asked "What kind of work was this person doing?" and "What were this person's most important activities or duties?" For respondents who return the paper form by mail, they write in a phrase in a provided box in response to each question. Those who do not return the survey form by mail are then subsampled for follow-up nonresponse conversion. Some receive a telephone interview, and some receive an in-person interview, during which they provide the same information and during which an interviewer can probe for clarity.

For the GSS, an in-person interview is usually conducted, but some interviews occur by telephone when the respondent refuses an in-person interview. After a series of screener questions to determine whether the respondent has a current job, the respondent is asked one of two questions: (1) "What kind of work do you normally do? That is, what is your job called?" or (2) "What kind of work did you normally do? That is, what was your job called?" The interviewer is then instructed to probe for clarity and elaboration and told "IF NOT ALREADY ANSWERED, ASK: What do you actually do in that job? Tell me what are some of your main duties?" or "IF NOT ALREADY ANSWERED, ASK: What did you actually do in that job? Tell me what were some of your main duties?"

Overall, the ACS and GSS are designed to prompt a respondent to recall similar information, but in somewhat different ways. The job description provided in the GSS interview has to pass an interviewer threshold of clarity, enhanced by guided followups, while for many ACS respondents this is not the case. In addition, the GSS elicits the occupation of the respondent, while the ACS elicits occupation from a household informant who is the survey respondent. Neither survey, without other labor force participation information, clarifies how recently the job elicited was held, if it is not currently being held.

Earnings. For the ACS, income is collected in a section on the form with introductory instructions: "Mark $(X)$ the 'Yes' box for each type of income this person received, and give your best estimate of the TOTAL AMOUNT during the PAST 12 MONTHS. (NOTE: The 'past 12 months' is the period from today's date one year ago up through today.)" ACS respondents are then asked to place digits in boxes for multiple 
categories, directly above the words "TOTAL AMOUNT for the past 12 months." The two standard components of personal earnings/income for the analysis of the ACS are the specific numerical values offered in response to two categories described on the form as "Wages, salary, commissions, bonuses, or tips from all jobs. Report amount before deductions for taxes, bonds, dues, or other items" and "Self-employment income from own nonfarm businesses or farm businesses, including proprietorships and partnerships. Report NET income after business expenses."

For the GSS, respondents are asked two income questions, after questions on household structure and on the number of earners in the family in the last calendar year (e.g., for 2013, during interviews conducted from spring through early fall of 2014). They are presented with a showcard with 25 income ranges, from (A) for (Y), for income intervals such as "Under $\$ 1,000$, , “\$35,000 to 39,999," and "\$150,000 or over." They are then instructed, as in the 2014 GSS: "In which of these groups did your total family income, from all sources, fall last year - 2013 - before taxes, that is. Just tell me the letter. Total income includes interest or dividends, rent, Social Security, other pensions, alimony or child support, unemployment compensation, public aid (welfare), armed forces or veteran's allotment." The respondent is then asked whether they earned any income in the prior calendar year from the job they identified earlier in the interview. If they answer yes, they are then asked "In which of these groups did your earnings from \{your job\}, from all sources for 2013 fall? That is, before taxes or other deductions. Just tell me the letter."

Altogether, the ACS personal earnings variable, and the GSS personal earnings/income variable differ in several ways. They refer to different 12-month intervals. The ACS is based on self-reported dollar values, while the GSS is based on a set of ordered earnings/income categories. Finally, the ACS asks about wages and salaries from all jobs in the immediately prior twelve months, whereas the GSS asks only about the primary job elicited earlier, and in the prior calendar year. The GSS, furthermore, gives no guidance on how self-employment income should be included, or not, in its personal earnings/income question, although this is likely the intent of the "all sources" language that concludes the survey item.

For the 10-class version of EGP, Table 5 presents the class distribution, mean years of education by class, and mean personal earnings by class for best-possible aligned samples drawn from a pooling of the 2012 and 2014 GSS and from a pooling of the 2012 and 2014 ACS. For the ACS, the sample composition is the same as for Table 1, but restricted to only 2012 and 2014 respondents who are not resident in group quarters (for samples sizes of 1,691,754 total for full-time, full-year workers and 2,717,746 for all workers). For the GSS, the sample was restricted to respondents in either 2012 or 2014, aged 25 to 64. For the GSS, "full-time, full year workers" are defined as having worked at least 50 weeks, usually full-time, in the previous calendar year, and reporting at least $\$ 10,623$ (in 2013 dollars, which is equivalent to $\$ 10,440$ in 2015 dollars) over the previous year ( $N=677$ in 2012 and $N=911$ in 2014). For the GSS, "all 
workers" are defined as having worked at least 1 week in the prior year, either full-time or parttime $(N=1,107$ in 2012 and $N=1,473$ in 2014).

For the ACS earnings values, we used the raw dollar values reported, adjusted to 2013 dollars (the calendar year prior to the last GSS wave, 2014). For the GSS, we needed to transform the categorical earnings variable, as detailed in the table just below. In brief, we selected midpoints of the categories and a Pareto multiplier for the top category (see Morgan and Tang 2007). We then adjusted the values for the 2012 GSS, which is the 2011 calendar year, to 2013 dollars. As a result, the mean values for earnings in the GSS are mean values only across the 50 plausible values below, which is far fewer values than for the ACS, where dollarby-dollar values are utilized.

\begin{tabular}{lrr} 
& $\begin{array}{r}\text { Assumed Value for } \\
\text { the 2012 GSS } \\
\text { (2011 reference year } \\
\text { in 2013 dollars) }\end{array}$ & $\begin{array}{c}\text { Assumed Value for } \\
\text { the 2014 GSS } \\
\text { (2013 reference year } \\
\text { in 2013 dollars) }\end{array}$ \\
Response Categories & 518 & 500 \\
\hline A. UNDER $\$ 1,000$ & 2,071 & 2,000 \\
B. $\$ 1,000$ to 2,999 & 3,625 & 3,500 \\
C. $\$ 3,000$ to 4,999 & 4,661 & 4,500 \\
D. $\$ 4,000$ to 5,999 & 5,697 & 5,500 \\
E. $\$ 5,000$ to 6,999 & 6,732 & 6,500 \\
F. $\$ 6,000$ to 7,999 & 7,768 & 7,500 \\
G. $\$ 7,000$ to 8,999 & 9,322 & 9,000 \\
H. $\$ 8,000$ to 9,999 & 11,652 & 11,250 \\
I. $\$ 10,000$ to 12,499 & 14,241 & 13,750 \\
J. $\$ 12,500$ to 14,999 & 16,831 & 16,250 \\
K. $\$ 15,000$ to 17,499 & 19,420 & 18,750 \\
L. $\$ 17,5000$ to 19,999 & 22,009 & 21,250 \\
M. $\$ 20,000$ to 22,499 & 24,599 & 23,750 \\
N. $\$ 22,500$ to 24,999 & 28,483 & 27,500 \\
O. $\$ 25,000$ to 29,999 & 33,661 & 32,500 \\
P. $\$ 30,000$ to 34,999 & 38,840 & 37,500 \\
Q. $\$ 30,000$ to 39,999 & 46,608 & 45,000 \\
R. $\$ 40,000$ to 49,999 & 56,965 & 55,000 \\
S. $\$ 50,000$ to 59,999 & 69,912 & 67,500 \\
T. $\$ 60,000$ to 74,999 & 85,448 & 100,000 \\
U. $\$ 75,000$ to 89,999 & 103,573 & 120,000 \\
V. $\$ 90,000$ to 109,999 & 124,288 & 140,000 \\
W. $\$ 110,000$ to 129,999 & 145,003 & 291,857 \\
X. $\$ 130,000$ to 149,999 & 302,286 & \\
Y. $\$ 150,000$ or over & & \\
& &
\end{tabular}


Table 5. Class Distributions, Education, and Earnings for a Comparison of EGP Classes (10-class version) in the 2012 and 2014 GSS and ACS

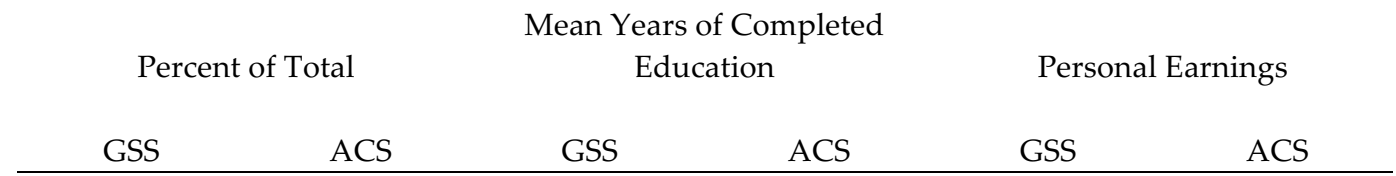

For full-time,

full-year

workers:

\begin{tabular}{|c|c|c|c|c|c|c|}
\hline I & 13.2 & 12.2 & 17.1 & 16.9 & 118,118 & 111,642 \\
\hline II & 19.5 & 19.9 & 15.9 & 15.7 & 87,572 & 78,428 \\
\hline IIIa & 18.4 & 18.2 & 14.5 & 13.9 & 57,367 & 53,175 \\
\hline IIIb & 14.2 & 13.7 & 13.3 & 13.1 & 38,551 & 39,836 \\
\hline IVc & 0.3 & 0.4 & $N<15$ & 13.1 & $N<15$ & 69,142 \\
\hline $\mathrm{V}$ & 9.9 & 10.5 & 13.7 & 13.8 & 57,901 & 63,984 \\
\hline VI & 6.2 & 7.1 & 12.6 & 12.3 & 51,967 & 48,714 \\
\hline VIIa & 15.6 & 17.2 & 11.8 & 11.9 & 41,241 & 37,529 \\
\hline VIIb & 0.3 & 0.5 & $N<15$ & 11.0 & $N<15$ & 33,156 \\
\hline Military & 2.5 & 0.3 & 14.5 & 14.4 & 66,077 & 60,805 \\
\hline \multicolumn{7}{|c|}{ For all workers: } \\
\hline I & 11.1 & 9.9 & 17.0 & 16.8 & 103,878 & 96,181 \\
\hline II & 17.8 & 17.4 & 16.0 & 15.7 & 72,287 & 64,758 \\
\hline IIIa & 16.7 & 18.0 & 14.5 & 13.9 & 47,538 & 39,490 \\
\hline IIIb & 17.3 & 17.8 & 13.3 & 13.0 & 27,697 & 25,005 \\
\hline IVc & 0.2 & 0.4 & $N<15$ & 13.0 & $N<15$ & 53,689 \\
\hline V & 9.1 & 9.2 & 13.8 & 13.8 & 52,217 & 52,039 \\
\hline VI & 6.4 & 6.8 & 12.4 & 12.2 & 46,007 & 38,016 \\
\hline VIIa & 19.2 & 19.6 & 11.5 & 11.8 & 30,058 & 25,760 \\
\hline VIIb & 0.3 & 0.7 & $N<15$ & 10.8 & $N<15$ & 21,292 \\
\hline Military & 2.0 & 0.2 & 14.1 & 14.3 & 56,586 & 51,318 \\
\hline
\end{tabular}

Source: American Community Surveys, 2012 and 2014 PUMS Data Files (extracted from the 2011-2015 file); General Social Survey 2012 and 2014 files (extracted from the 1972-2014 cumulative file, release 6)

Notes: For both the ACS and the GSS, dollar values were adjusted to 2013 dollars. For the ACS, the sample composition is the same as for Table 1, but restricted to only 2012 and 2014 respondents who are not resident in group quarters (for samples sizes of 1,691,754 total for full-time, full-year workers and 2,717,746 for all workers). For the GSS, the sample was restricted to respondents in either 2012 or 2014, aged 25 to 64. For the GSS, "full-time, full year workers" are defined as having worked at least 50 weeks, usually full-time, in the previous calendar year, and reporting at least $\$ 10,623$ (in 2013 dollars, which is equivalent to $\$ 10,440$ in 2015 dollars) over the previous year ( $N=$ 677 in 2012 and $N=911$ in 2014). For the GSS, "all workers" are defined as having worked at least 1 week in the prior year, either full-time or part-time ( $N=1,107$ in 2012 and $N=1,473$ in 2014). For the GSS, we did not impute itemspecific missing data (in the "all worker" sample: 1 case for education and 165 cases for earnings). The GSS sample weight (wtssnr) was adjusted to give respondents from 2012 larger weights to compensate for its smaller size relative to 2014. Cells were left blank if the sample size was less than 15 (i.e., $N<15$ ). 
Remember that the GSS samples are much smaller (2,580 workers in this age range, of whom only 1,588 are full-time, full-year workers) than the ACS samples (2,717,746 workers in this age range, of whom only 1,691,754 are full-time, full-year workers). Indeed, the ACS sample is the equivalent of more than 1,000 GSS samples. Even so, the alignment between the GSS and ACS results in Table 5 is reassuring.

Consider first the distributions of the 10 classes in columns 1 and 2, which are very similar. Both surveys appear to be collecting occupation information from the same population, and coding that information in the same way. The largest differences that exist (e.g., for classes I, VIIa, and for the military) are consistent with expected sampling variation in the GSS. And the mismatch is larger when the sample is narrowed to full-time, full-year workers. This is a smaller sample, and the definition of "full-time, full-year" differs a bit across the two surveys, based on the reference period utilized, as well as full-time items (a question on usual hours for the ACS, but a question on usually full-time for the GSS). Nonetheless, we cannot know whether some differences are systematic (as could be the case, for example, if the ACS uses poststratification to generate a lower rate of "Military" occupations).

Similarly, the mean education of each of these classes suggests that the occupational incumbents have similar education levels. There is more variation in personal earnings across classes, with the GSS typically delivering higher levels. Nonetheless, as explained above, the questions that elicit the information have substantial differences.

To probe further, Table 6 presents demographic characteristics for the plausibly aligned GSS and ACS samples, analogous to those presented above in Table 3. Again, we see a great deal of consistency in how the coding functions in both the GSS and the ACS. 
Table 6. Selected Demographic Characteristics for a Comparison of EGP Classes (10-class version) in the 2012 and 2014 GSS and ACS

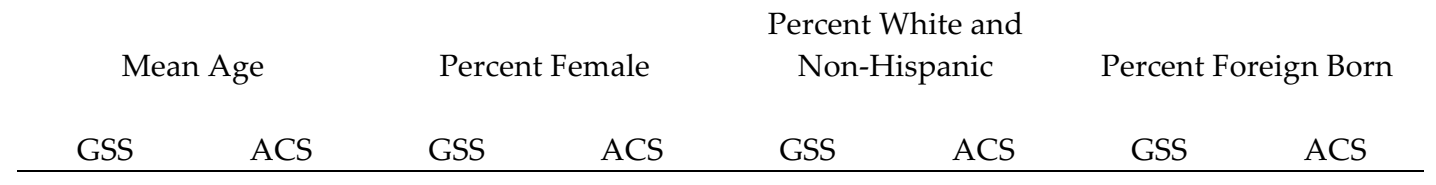

For full-time.

full-year

workers:

\begin{tabular}{|c|c|c|c|c|c|c|c|c|}
\hline I & 45.0 & 44.2 & 35.6 & 37.9 & 73.4 & 74.0 & 22.7 & 18.7 \\
\hline II & 45.2 & 43.6 & 53.4 & 53.6 & 76.4 & 75.5 & 12.7 & 12.0 \\
\hline IIIa & 43.1 & 44.2 & 69.1 & 67.3 & 65.5 & 70.4 & 12.2 & 10.5 \\
\hline $\mathrm{IIIb}$ & 41.5 & 42.4 & 61.7 & 57.8 & 66.8 & 60.2 & 11.8 & 20.0 \\
\hline IVc & $N<15$ & 47.2 & $N<15$ & 9.6 & $N<15$ & 91.3 & $N<15$ & 7.0 \\
\hline V & 44.1 & 43.3 & 33.3 & 26.0 & 68.4 & 73.0 & 14.5 & 12.1 \\
\hline VI & 45.6 & 43.5 & 6.0 & 4.3 & 54.2 & 68.9 & 16.9 & 19.3 \\
\hline VIIa & 45.0 & 43.7 & 28.8 & 23.7 & 50.9 & 52.0 & 25.4 & 29.5 \\
\hline VIIb & $N<15$ & 42.0 & $N<15$ & 18.4 & $N<15$ & 40.5 & $N<15$ & 49.9 \\
\hline Military & 39.4 & 34.7 & 18.9 & 10.2 & 64.5 & 69.9 & 7.3 & 5.9 \\
\hline \multicolumn{9}{|l|}{$\begin{array}{l}\text { For all } \\
\text { workers: }\end{array}$} \\
\hline I & 44.7 & 44.4 & 38.5 & 41.8 & 73.7 & 73.7 & 24.2 & 19.2 \\
\hline II & 45.0 & 44.2 & 60.4 & 58.5 & 77.6 & 75.8 & 12.2 & 11.9 \\
\hline IIIa & 43.1 & 44.4 & 71.4 & 71.5 & 63.6 & 70.5 & 12.7 & 11.1 \\
\hline IIIlb & 40.7 & 42.2 & 69.0 & 67.3 & 62.3 & 61.1 & 14.0 & 18.9 \\
\hline IVc & $N<15$ & 47.8 & $N<15$ & 15.8 & $N<15$ & 89.6 & $N<15$ & 8.1 \\
\hline V & 43.8 & 43.8 & 36.4 & 30.7 & 66.3 & 72.9 & 15.4 & 12.8 \\
\hline VI & 44.7 & 44.1 & 6.9 & 5.2 & 60.1 & 68.5 & 16.8 & 19.3 \\
\hline VIIa & 43.8 & 43.5 & 36.4 & 30.4 & 46.1 & 52.2 & 26.4 & 28.0 \\
\hline VIIb & $N<15$ & 42.2 & $N<15$ & 30.8 & $N<15$ & 36.6 & $N<15$ & 52.1 \\
\hline Military & 39.6 & 34.9 & 16.9 & 11.6 & 55.9 & 69.0 & 7.2 & 5.9 \\
\hline
\end{tabular}

Source: See Table 5.

Notes: For the sample compositions, see the notes to Table 5. Cells were left blank if the sample size was less than 15 (i.e., $N<15)$. 
Tables 5 and 6 demonstrate that the 10-class version of the EGP coding delivers similar results for the GSS and for the ACS. What about the 11 and 12 class versions? As explained above, the 11-class version of EGP has one additional class, which is class IVab, and which represents all self-employed individuals in the occupations assigned to classes IIIa, IIIb, V, VI, and VIIa for the 10-class version of EGP. The 12-class version of EGP separates class IVab into classes IVa and IVb, with the former compromising the subset of individuals who report having employees. ${ }^{9}$

For the both the ACS and GSS, it is possible to code the 11-class version. For the GSS, it is also possible to code the 12-class version from 2004 onward. Self-employment is embedded within the ACS "class of worker" item, but it is a separate item on the GSS. Table 7 reveals the difference in a comparison of the GSS to the ACS, again using the 10-class version of EGP. The percent of class members who are employed by private employers, public employers, and who are self-employed adds to 100 percent (subject to rounding) for the ACS but not for the GSS. Accordingly, the "private employer" designation is more common in the GSS, since it includes many self-employed individuals. In addition, it would appear that more individuals report being self-employed in the GSS. It is unclear whether these differences reflect the different structures of the items, or whether they are complicated by individuals holding more than one job.

Table 8, which has the same structure as Table 7, allocates individuals to the 11-class version of EGP (and for the GSS, also the 12-class version of EGP). As a result, various "All" and "None" entries are introduced as structural zeroes (based on definitions, not realized samples of zero). With the implementation of the 11-class and 12-class versions of EGP shown in Table 8, Tables 9 and 10 present the same outcomes analyzed, respectively, for Tables 5 and 6. With the caveat that GSS-ACS comparisons are more difficult because of differences in selfemployment rates across the surveys, we continue to see considerable similarity across both data sources.

\footnotetext{
${ }^{9}$ In some realizations of the EGP class schema, class IVa includes self-employed individuals who have 10 or more employees while class IVb includes self-employed individuals who have fewer than 10 employees. I have implemented the 1-or-more cutoff instead, under the assumption that individuals who have many employees are more likely to select class I occupations, such as Chief Executive, which never end up in either class IVa or class IVb because of the first-stage coding of the 10-class schema.
} 
Table 7. Type of Employment for a Comparison of EGP Classes (10-class version) in the 2012 and 2014 GSS and ACS

\begin{tabular}{|c|c|c|c|c|c|c|c|}
\hline \multicolumn{2}{|c|}{$\begin{array}{c}\text { Percent Private } \\
\text { Employer }\end{array}$} & \multicolumn{2}{|c|}{$\begin{array}{l}\text { Percent Public } \\
\text { Employer }\end{array}$} & \multicolumn{2}{|c|}{$\begin{array}{l}\text { Percent Self- } \\
\text { Employed }\end{array}$} & \multicolumn{2}{|c|}{$\begin{array}{c}\text { Percent Full-time, } \\
\text { Full-year }\end{array}$} \\
\hline GSS & ACS & GSS & ACS & GSS & ACS & GSS & ACS \\
\hline
\end{tabular}

For full-time,

full-year

workers:

\begin{tabular}{|c|c|c|c|c|c|c|c|c|}
\hline I & 66.0 & 71.2 & 22.2 & 18.9 & 11.8 & 9.9 & All & All \\
\hline II & 71.9 & 69.4 & 22.2 & 25.4 & 5.9 & 5.2 & All & All \\
\hline IIIa & 75.9 & 77.7 & 15.3 & 17.3 & 8.7 & 5.0 & All & All \\
\hline IIIb & 74.4 & 82.2 & 8.0 & 7.1 & 17.5 & 10.7 & All & All \\
\hline IVc & $N<15$ & 22.5 & $N<15$ & 0.8 & $N<15$ & 76.7 & All & All \\
\hline V & 60.9 & 65.4 & 23.2 & 25.4 & 15.9 & 9.2 & All & All \\
\hline VI & 72.0 & 79.1 & 15.6 & 9.5 & 12.4 & 11.4 & All & All \\
\hline VIIa & 83.2 & 85.3 & 9.1 & 8.4 & 7.6 & 6.3 & All & All \\
\hline VIIb & $N<15$ & 87.0 & $N<15$ & 4.2 & $N<15$ & 8.8 & All & All \\
\hline Military & 16.0 & 0.0 & 84.0 & 100.0 & 0.0 & 0.0 & All & All \\
\hline \multicolumn{9}{|l|}{$\begin{array}{l}\text { For all } \\
\text { workers: }\end{array}$} \\
\hline I & 63.4 & 68.7 & 22.6 & 19.5 & 14.0 & 11.8 & 72.9 & 77.5 \\
\hline II & 63.1 & 66.2 & 29.4 & 27.2 & 7.4 & 6.7 & 67.1 & 71.9 \\
\hline IIIa & 73.8 & 76.0 & 16.1 & 17.1 & 10.0 & 6.9 & 67.2 & 64.0 \\
\hline IIIb & 78.1 & 81.1 & 9.2 & 7.2 & 12.8 & 11.7 & 50.8 & 48.5 \\
\hline IVc & $N<15$ & 20.0 & $N<15$ & 0.7 & $N<15$ & 79.3 & $N<15$ & 67.3 \\
\hline V & 58.1 & 64.5 & 19.2 & 22.0 & 22.5 & 13.5 & 66.4 & 71.9 \\
\hline VI & 71.0 & 76.3 & 12.0 & 7.7 & 16.9 & 16.0 & 60.1 & 65.5 \\
\hline VIIa & 78.5 & 83.5 & 10.0 & 7.3 & 11.3 & 9.2 & 50.5 & 55.3 \\
\hline VIIb & $N<15$ & 87.0 & $N<15$ & 2.8 & $N<15$ & 10.2 & $N<15$ & 46.7 \\
\hline Military & 26.7 & 0.0 & 73.3 & 100.0 & 0.0 & 0.0 & 75.5 & 79.2 \\
\hline
\end{tabular}

Source: See Table 5.

Notes: For the sample compositions, see the notes to Table 5. Cells were left blank if the sample size was less than 15 (i.e., $N<15$ ). For the GSS, we did not impute item-specific missing data (in the "all worker" sample: 11 cases for private/public employer, 2 cases for self-employment, and 21 cases for full-time, full-year). 
Table 8. Type of Employment for a Comparison of EGP Classes (11-class and 12-class versions) in the 2012 and 2014 GSS and ACS

\begin{tabular}{|c|c|c|c|c|c|c|c|}
\hline \multicolumn{2}{|c|}{$\begin{array}{l}\text { Percent Private } \\
\text { Employer }\end{array}$} & \multicolumn{2}{|c|}{$\begin{array}{l}\text { Percent Public } \\
\text { Employer }\end{array}$} & \multicolumn{2}{|c|}{$\begin{array}{l}\text { Percent Self- } \\
\text { Employed }\end{array}$} & \multicolumn{2}{|c|}{$\begin{array}{l}\text { Percent Full-time, } \\
\text { Full-year }\end{array}$} \\
\hline GSS & ACS & GSS & ACS & GSS & ACS & GSS & ACS \\
\hline
\end{tabular}

For full-time,

full-year

workers:

\begin{tabular}{|c|c|c|c|c|c|c|c|c|}
\hline I & 66.0 & 71.2 & 22.2 & 18.9 & 11.8 & 9.9 & All & All \\
\hline II & 71.9 & 69.4 & 22.2 & 25.4 & 5.9 & 5.2 & All & All \\
\hline IIIa & 83.2 & 81.8 & 16.8 & 18.2 & None & None & All & All \\
\hline $\mathrm{IIIb}$ & 90.2 & 92.0 & 9.8 & 8.0 & None & None & All & All \\
\hline IVab & None & None & None & None & All & All & All & All \\
\hline IVa & None & -- & None & -- & All & -- & All & All \\
\hline $\mathrm{IVb}$ & None & -- & None & -- & All & -- & All & All \\
\hline IVc & $N<15$ & 22.5 & $N<15$ & 0.8 & $N<15$ & 76.7 & All & All \\
\hline $\mathrm{V}$ & 72.4 & 72.1 & 27.6 & 27.9 & None & None & All & All \\
\hline VI & 82.2 & 89.3 & 17.8 & 10.7 & None & None & All & All \\
\hline VIIa & 90.1 & 85.3 & 9.9 & 8.4 & None & None & All & All \\
\hline VIIb & $N<15$ & 87.0 & $N<15$ & 4.2 & $N<15$ & 8.8 & All & All \\
\hline Military & 16.0 & 0.0 & 84.0 & 100.0 & 0.0 & 0.0 & All & All \\
\hline \multicolumn{9}{|l|}{$\begin{array}{l}\text { For all } \\
\text { workers: }\end{array}$} \\
\hline I & 63.4 & 68.7 & 22.6 & 19.5 & 14.0 & 11.8 & 72.9 & 77.5 \\
\hline II & 63.1 & 66.2 & 29.4 & 27.2 & 7.4 & 6.7 & 67.1 & 71.9 \\
\hline IIIIa & 82.0 & 81.6 & 18.0 & 18.4 & None & None & 68.1 & 65.2 \\
\hline $\mathrm{IIIlb}$ & 89.5 & 91.9 & 10.5 & 8.1 & None & None & 48.1 & 49.1 \\
\hline IVab & None & None & None & None & All & All & 50.9 & 44.2 \\
\hline IVa & None & -- & None & -- & All & -- & 68.7 & -- \\
\hline $\mathrm{IVb}$ & None & -- & None & -- & All & -- & 44.7 & -- \\
\hline IVc & $N<15$ & 20.0 & $N<15$ & 0.7 & $N<15$ & 79.3 & $N<15$ & 67.3 \\
\hline $\mathrm{V}$ & 75.2 & 74.5 & 24.8 & 25.5 & None & None & 63.2 & 75.4 \\
\hline VI & 85.5 & 90.0 & 14.5 & 9.1 & None & None & 52.7 & 65.5 \\
\hline VIIa & 88.7 & 92.0 & 11.3 & 8.0 & None & None & 61.5 & 55.3 \\
\hline VIIb & $N<15$ & 87.0 & $N<15$ & 2.8 & $N<15$ & 10.2 & $N<15$ & 46.7 \\
\hline Military & 26.7 & 0.0 & 73.3 & 100.0 & 0.0 & 0.0 & 75.5 & 79.2 \\
\hline
\end{tabular}

Source: See Table 5.

Notes: See Table 7. 
Table 9. Class Distributions, Education, and Earnings for a Comparison of EGP Classes (11-class and 12-class versions) in the 2012 and 2014 GSS and ACS

\begin{tabular}{cccccc}
\multicolumn{2}{c}{ Percent of Total } & \multicolumn{2}{c}{$\begin{array}{c}\text { Mean Years of Completed } \\
\text { Education }\end{array}$} & \multicolumn{2}{c}{ Personal Earnings } \\
GSS & ACS & GSS & ACS & GSS & ACS \\
\hline
\end{tabular}

For full-time,

full-year

workers:

\begin{tabular}{lrrrrrr} 
I & 13.2 & 12.2 & 17.1 & 16.9 & 118,118 & 111,642 \\
II & 19.5 & 19.9 & 15.9 & 15.7 & 87,572 & 78,428 \\
IIIa & 16.8 & 17.3 & 14.4 & 13.9 & 49,420 & 51,950 \\
IIIb & 11.7 & 12.3 & 13.2 & 13.1 & 35,365 & 38,683 \\
IVab & 7.6 & 5.2 & 13.2 & 13.2 & 74,509 & 57,614 \\
\multicolumn{1}{c}{ IVa } & 2.7 & -- & 13.4 & -- & 111,470 & -- \\
$\quad$ IVb & 5.0 & -- & 13.1 & -- & 52,027 & -- \\
IVc & 0.3 & 0.4 & $N<15$ & 13.1 & $N<15$ & 69,142 \\
V & 8.3 & 9.5 & 13.9 & 13.8 & 57,799 & 63,053 \\
VI & 5.4 & 6.3 & 12.9 & 12.3 & 55,762 & 48,981 \\
VIIa & 14.4 & 16.1 & 11.8 & 11.9 & 39,496 & 36,893 \\
VIIb & 0.3 & 0.5 & $N<15$ & 11.0 & $N<15$ & 33,156 \\
Military & 2.5 & 0.3 & 14.5 & 14.4 & 66,077 & 60,805
\end{tabular}

For all workers:

\begin{tabular}{lrrrrrr} 
I & 11.1 & 9.9 & 17.0 & 16.8 & 103,878 & 96,181 \\
II & 17.8 & 17.4 & 16.0 & 15.7 & 72,287 & 64,758 \\
IIIa & 15.0 & 16.7 & 14.4 & 13.8 & 42,381 & 38,971 \\
IIIb & 15.1 & 15.7 & 13.2 & 13.0 & 25,148 & 24,467 \\
IVab & 9.2 & 7.5 & 13.3 & 13.1 & 52,557 & 34,666 \\
$\quad$ IVa & 2.4 & -- & 13.6 & -- & 95,089 & -- \\
$\quad$ IVb & 6.8 & -- & 13.2 & -- & 36,971 & -- \\
IVc & 0.2 & 0.4 & $N<15$ & 13.0 & $N<15$ & 53,689 \\
V & 7.0 & 8.0 & 14.0 & 13.8 & 53,013 & 52,660 \\
VI & 5.3 & 5.7 & 12.4 & 12.2 & 46,837 & 39,396 \\
VIIa & 17.0 & 17.8 & 11.5 & 11.8 & 29,413 & 25,715 \\
VIIb & 0.3 & 0.7 & $N<15$ & 10.8 & $N<15$ & 21,292 \\
Military & 2.0 & 0.2 & 14.1 & 14.3 & 56,586 & 51,318 \\
\hline
\end{tabular}

Source: See Table 5.

Notes: See Table 5. 
Table 10. Selected Demographic Characteristics for a Comparison of EGP Classes (11-class and 12-class versions) in the 2012 and 2014 GSS and ACS

\begin{tabular}{|c|c|c|c|c|c|c|c|}
\hline \multicolumn{2}{|c|}{ Mean Age } & \multicolumn{2}{|c|}{ Percent Female } & \multicolumn{2}{|c|}{$\begin{array}{l}\text { Percent White and } \\
\text { Non-Hispanic }\end{array}$} & \multicolumn{2}{|c|}{ Percent Foreign Born } \\
\hline GSS & ACS & GSS & ACS & GSS & ACS & GSS & ACS \\
\hline
\end{tabular}

For full-time,

full-year

workers:

\begin{tabular}{lrrrrrrrr} 
I & 45.0 & 44.2 & 35.6 & 37.9 & 73.4 & 74.0 & 22.7 & 18.7 \\
II & 45.2 & 43.6 & 53.4 & 53.6 & 76.4 & 75.5 & 12.7 & 12.0 \\
IIIa & 42.5 & 43.9 & 69.9 & 68.5 & 63.6 & 69.8 & 12.9 & 10.3 \\
IIIb & 41.0 & 41.9 & 63.8 & 58.6 & 64.8 & 59.7 & 11.9 & 19.0 \\
IVab & 46.8 & 46.6 & 40.5 & 29.6 & 73.3 & 70.7 & 19.2 & 24.3 \\
\multicolumn{1}{c}{ IVa } & 46.8 & -- & 34.6 & -- & 71.1 & -- & 22.9 & -- \\
$\quad$ IVb & 46.8 & -- & 43.6 & -- & 74.5 & -- & 17.2 & -- \\
IVc & $N<15$ & 47.2 & $N<15$ & 9.6 & $N<15$ & 91.3 & $N<15$ & 7.0 \\
V & 43.3 & 43.0 & 35.3 & 26.8 & 67.4 & 72.3 & 12.7 & 11.6 \\
VI & 45.7 & 43.2 & 5.1 & 4.3 & 54.9 & 68.1 & 14.3 & 19.0 \\
VIIa & 44.7 & 43.6 & 28.6 & 24.2 & 49.7 & 51.6 & 24.3 & 29.0 \\
VIIb & $N<15$ & 42.0 & $N<15$ & 18.4 & $N<15$ & 40.5 & $N<15$ & 49.4 \\
Military & 39.4 & 34.7 & 18.9 & 10.2 & 64.5 & 69.9 & 7.3 & 5.9
\end{tabular}

For all

workers:

\begin{tabular}{lrrrrrrrr} 
I & 44.7 & 44.4 & 38.5 & 41.8 & 73.7 & 73.7 & 24.2 & 19.2 \\
II & 45.0 & 44.2 & 60.4 & 58.5 & 77.6 & 75.8 & 12.2 & 11.9 \\
IIIa & 42.7 & 44.1 & 71.9 & 72.5 & 61.6 & 69.8 & 13.1 & 10.8 \\
IIIb & 40.0 & 41.7 & 71.3 & 67.5 & 61.0 & 60.7 & 14.3 & 18.0 \\
IVab & 46.7 & 46.6 & 45.2 & 40.9 & 68.0 & 68.9 & 19.9 & 24.0 \\
$\quad$ IVa & 46.3 & -- & 32.6 & -- & 74.9 & -- & 15.7 & -- \\
$\quad$ IVb & 46.8 & -- & 49.5 & -- & 65.6 & -- & 21.4 & -- \\
IVc & $N<15$ & 47.8 & $N<15$ & 15.8 & $N<15$ & 89.6 & $N<15$ & 8.1 \\
V & 43.1 & 43.3 & 38.5 & 31.1 & 66.1 & 72.0 & 12.4 & 12.1 \\
VI & 44.1 & 43.5 & 7.2 & 5.1 & 59.2 & 67.4 & 14.1 & 19.1 \\
VIIa & 43.3 & 43.3 & 34.1 & 30.4 & 44.7 & 51.7 & 26.6 & 27.2 \\
VIIb & $N<15$ & 42.2 & $N<15$ & 30.8 & $N<15$ & 36.6 & $N<15$ & 52.1 \\
Military & 39.6 & 34.9 & 16.9 & 11.6 & 55.9 & 69.0 & 7.2 & 5.9 \\
\hline
\end{tabular}

Source: See Table 5.

Notes: See Table 6. 


\section{Sample Stata Code for Merging the .csv File and the GSS Cumulative File}

The following Stata code demonstrates how to code the 10,11, and 12-class versions of EGP in the GSS cumulative file, by first merging in the crosswalk from the .csv file named occ10-to-egp-class-crosswalk.csv. The syntax also contains basic tabulate commands to demonstrate what the code accomplishes. This code can be copied directly from this pdf file, and it is also available in the text file named code-for-egp-crosswalk.do, which is available along with the .csv file.

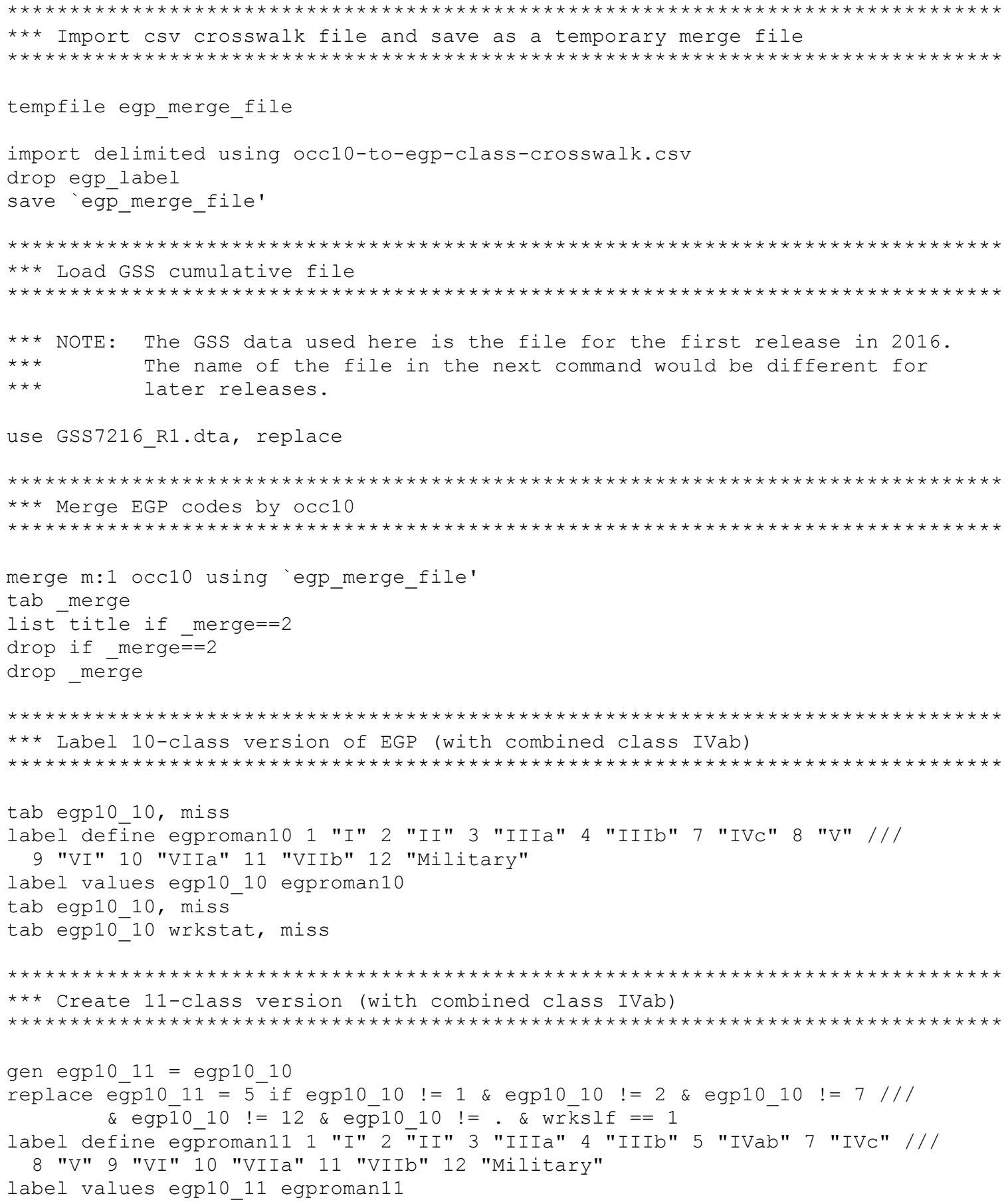




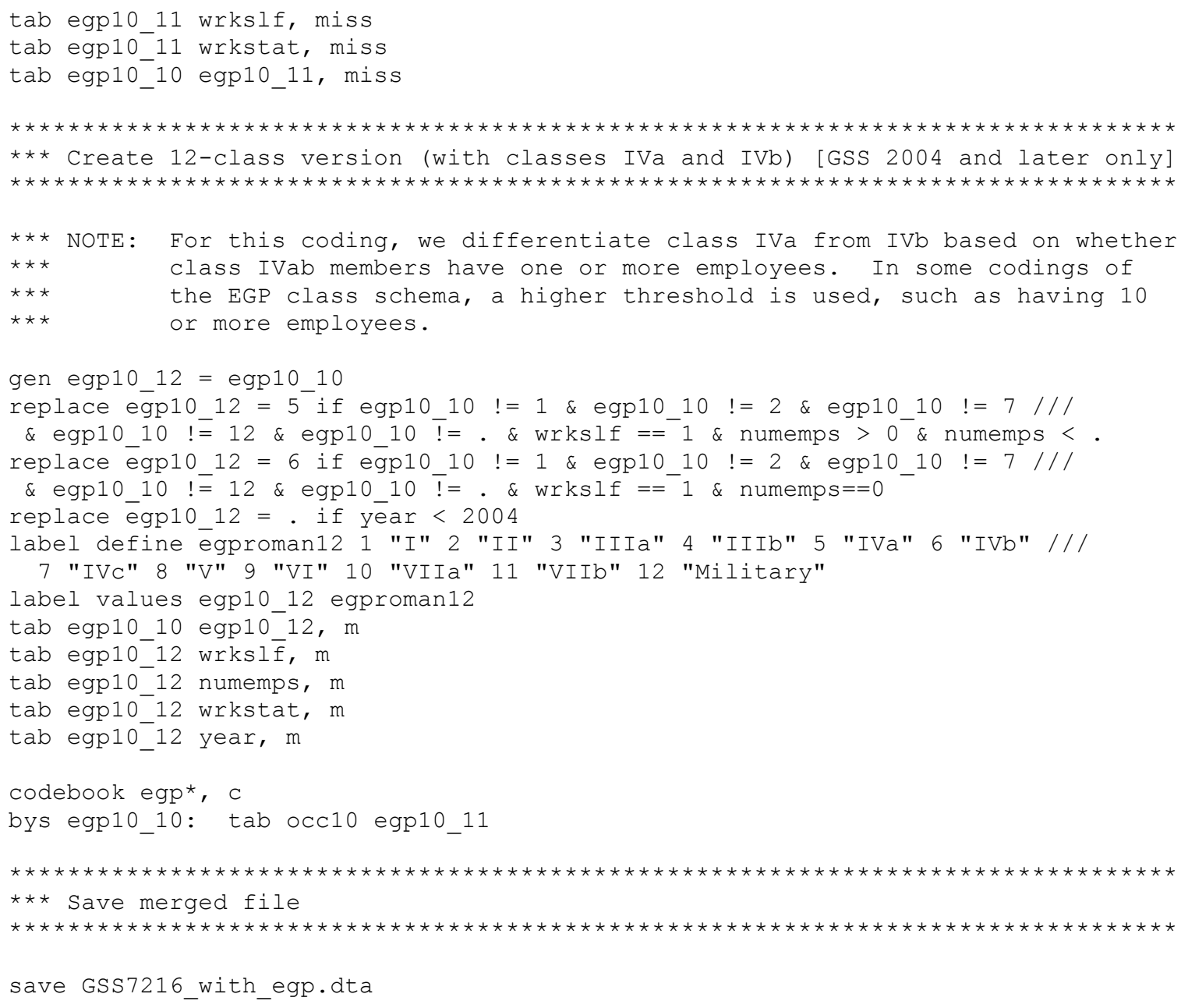




\section{Conclusions}

As discussed in the first pages of this report, principled reasons exist to eschew all usage of the EGP class schema, in its original form or in this new coding. If one accepts that EGP is a worthwhile measure to have, the value of this particular coding depends in part on its rationale and in part on its future demonstrated value in empirical research. I have endeavored to explain the full set of coding decisions taken so that potential users can evaluate whether to adopt this coding for their own research projects. I have also offered a detailed analysis that compares the coding when used in the ACS and the GSS, to the extent that such a comparison is possible. This analysis should generate confidence that the coding can be used to advance research in many domains, but that confidence awaits full justification from new topical research.

Nonetheless, rather than include the new EGP variable as an official GSS variable, I am instead posting the merge file to the GSS website to facilitate considered adoption in whole, or partial adoption based on the user's own tailoring. The associated files can be easily revised before being merged into the GSS. A few words of caution are in order for those inclined to revise: Occupations are not always interpretable without first consulting the SOC and its underlying descriptions of job activities and job titles. In addition, lay notions of what occupations would seem to be do not always match the characteristics of occupational incumbents, or the nature of the work performed. Finally, the GSS itself is too small to yield precise estimates of the characteristics of occupational incumbents, and therefore a larger data source such as the ACS must be consulted when changes are considered. 


\section{References Cited}

Erikson, Robert and John H. Goldthorpe. 1985. "Are American Rates of Social Mobility

Exceptionally High? New Evidence on an Old Issue." European Sociological Review 1:1-22.

-. 1992. The Constant Flux: A Study of Class Mobility in Industrial Societies. Oxford: Oxford University Press.

Erikson, Robert, John H. Goldthorpe, and Lucienne Portocarero. 1979. "Intergenerational Class Mobility in Three Western European Societies: England, France and Sweden." The British Journal of Sociology 30:415-41.

-. 1982. "Social Fluidity in Industrial Nations: England, France and Sweden." The British Journal of Sociology 33:1-34.

-. 1983. "Intergenerational Class Mobility and the Convergence Thesis: England, France and Sweden." The British Journal of Sociology 34:303-43.

Goldthorpe, John H. 1981. 1987. Social Mobility and Class Structure in Modern Britain. Oxford: Clarendon Press.

Grusky, David B. and Jesper B. Sørensen. 1998. “Can Class Analysis Be Salvaged?” American Journal of Sociology 103:1187-234.

Hout, Michael, Tom W. Smith, and Peter V. Marsden. 2016. "Prestige and Socioeconomic Scores for the 2010 Census Codes." Chicago, Illinois: GSS Methodological Report No. 124, National Opinion Research Center.

Kingston, Paul W. 2000. The Classless Society. Stanford: Stanford University Press.

Morgan, Stephen L. and Mark W. McKerrow. 2004. "Social Class, Rent Destruction, and the Earnings of Black and White Men, 1982-2000." Research in Social Stratification and Mobility 21:215-51.

Morgan, Stephen L. and Zun Tang. 2007. “Social Class and Workers' Rent, 1983-2001." Research in Social Stratification and Mobility 25:273-93.

Pakulski, Jan and Malcolm Waters. 1996. The Death of Class. Thousand Oaks: Sage.

Scopp, Thomas S. 2003. "The Relationship between the 1990 Census and Census 2000 Industry and Occupation Classification Systems." Washington, DC: Technical Paper \#65, U.S. Census Bureau.

Smith, Tom W. and Jaesok Son. 2014. "Measuring Occupational Prestige in the 2012 General Social Survey." Chicago, Illinois: GSS Methodological Report No. 122, National Opinion Research Center.

Weeden, Kim A. and David B. Grusky. 2005. “The Case for a New Class Map.” American Journal of Sociology 111:141-212.

Wright, Erik Olin. 1997. Class Counts: Comparative Studies in Class Analysis. Cambridge: Cambridge University Press. 


\section{Appendix Tables}

Table A1. GSS Occupational Distributions for the 10-Class Version of EGP (i.e., no Class IVa or Class IVb)

\begin{tabular}{|c|c|c|c|c|c|c|c|}
\hline Class & Occupational Title (ACS 2012) & $\begin{array}{l}\text { ACS } \\
2012 \\
\end{array}$ & $\begin{array}{r}\text { GSS } \\
\text { occ10 } \\
\end{array}$ & $\begin{array}{l}\text { Recoded } \\
\text { to ACS }\end{array}$ & $\begin{array}{r}\text { GSS } \\
1994- \\
2000 \\
\end{array}$ & $\begin{array}{r}\text { GSS } \\
2002- \\
2008 \\
\end{array}$ & $\begin{array}{r}\text { GSS } \\
2010- \\
2016 \\
\end{array}$ \\
\hline I & Accountants And Auditors & 800 & 800 & & 112 & 120 & 93 \\
\hline I & Actuaries & 1200 & 1200 & & 2 & 1 & 0 \\
\hline $\mathrm{I}$ & Aerospace Engineers & 1320 & 1320 & & 7 & 19 & 7 \\
\hline I & Agricultural And Food Scientists & 1600 & 1600 & & 5 & 2 & 4 \\
\hline I & Architects, Except Naval & 1300 & 1300 & & 20 & 16 & 14 \\
\hline I & Architectural And Engineering Managers & 300 & 300 & & 9 & 11 & 4 \\
\hline I & Astronomers And Physicists & 1700 & 1700 & & 0 & 1 & 0 \\
\hline I & Atmospheric And Space Scientists & 1710 & 1710 & & 2 & 0 & 2 \\
\hline I & Biological Scientists & 1610 & 1610 & & 6 & 11 & 8 \\
\hline I & Budget Analysts & 820 & 820 & & 2 & 7 & 3 \\
\hline I & Chemical Engineers & 1350 & 1350 & & 10 & 7 & 2 \\
\hline I & Chemists And Materials Scientists & 1720 & 1720 & & 9 & 12 & 5 \\
\hline I & Chief Executives And Legislators & 10 & 10 & & 74 & 62 & 64 \\
\hline I & Chief Executives And Legislators & 10 & 30 & $\mathrm{x}$ & 1 & 4 & 1 \\
\hline $\mathrm{I}$ & Chiropractors & 3000 & 3000 & & 0 & 9 & 3 \\
\hline I & Civil Engineers & 1360 & 1360 & & 25 & 29 & 19 \\
\hline \multirow[t]{2}{*}{ I } & Computer And Information Research & & & & & & \\
\hline & Scientists & 1005 & 1005 & & 6 & 2 & 0 \\
\hline \multirow[t]{2}{*}{ I } & Computer And Information Systems & & & & & & \\
\hline & Managers & 110 & 110 & & 18 & 20 & 25 \\
\hline I & Computer Hardware Engineers & 1400 & 1400 & & 4 & 5 & 2 \\
\hline I & Conservation Scientists And Foresters & 1640 & 1640 & & 3 & 6 & 3 \\
\hline I & Dentists & 3010 & 3010 & & 10 & 7 & 6 \\
\hline I & Economists & 1800 & 1800 & & 1 & 1 & 3 \\
\hline I & Education Administrators & 230 & 230 & & 44 & 65 & 52 \\
\hline I & Electrical And Electronics Engineers & 1410 & 1410 & & 35 & 29 & 22 \\
\hline I & Environmental Engineers & 1420 & 1420 & & 7 & 8 & 3 \\
\hline I & Environmental Scientists And Geoscientists & 1740 & 1740 & & 4 & 7 & 11 \\
\hline I & Financial Analysts & 840 & 840 & & 3 & 8 & 9 \\
\hline \multirow[t]{2}{*}{ I } & Industrial Engineers, Including Health And & & & & & & \\
\hline & Safety & 1430 & 1430 & & 20 & 24 & 18 \\
\hline I & Judicial Law Clerks & 2105 & 2105 & & 0 & 1 & 2 \\
\hline I & $\begin{array}{l}\text { Lawyers, And Judges, Magistrates, And Other } \\
\text { Judicial Workers }\end{array}$ & 2100 & 2100 & & 51 & 62 & 46 \\
\hline \multirow[t]{2}{*}{$\mathrm{I}$} & Lawyers, And Judges, Magistrates, And Other & & & & & & \\
\hline & Judicial Workers & 2100 & 2110 & $\mathrm{x}$ & 7 & 5 & 1 \\
\hline I & Management Analysts & 710 & 710 & & 45 & 56 & 44 \\
\hline I & Marine Engineers And Naval Architects & 1440 & 1440 & & 0 & 1 & 0 \\
\hline I & Materials Engineers & 1450 & 1450 & & 1 & 2 & 2 \\
\hline $\mathrm{I}$ & Mechanical Engineers & 1460 & 1460 & & 25 & 34 & 28 \\
\hline
\end{tabular}




\begin{tabular}{|c|c|c|c|c|c|c|c|}
\hline Class & Occupational Title (ACS 2012) & $\begin{array}{l}\text { ACS } \\
2012\end{array}$ & $\begin{array}{r}\text { GSS } \\
\text { occ10 }\end{array}$ & $\begin{array}{l}\text { Recoded } \\
\text { to ACS }\end{array}$ & $\begin{array}{r}\text { GSS } \\
1994- \\
2000 \\
\end{array}$ & $\begin{array}{r}\text { GSS } \\
2002- \\
2008 \\
\end{array}$ & $\begin{array}{r}\text { GSS } \\
2010- \\
2016 \\
\end{array}$ \\
\hline I & Medical And Health Services Managers & 350 & 350 & & 37 & 30 & 45 \\
\hline \multirow[t]{2}{*}{ I } & Medical Scientists, And Life Scientists, All & & & & & & \\
\hline & Other & 1650 & 1650 & & 4 & 6 & 12 \\
\hline \multirow[t]{2}{*}{ I } & Miscellaneous Engineers, Including Nuclear & & & & & & \\
\hline & Engineers & 1530 & 1530 & & 9 & 13 & 10 \\
\hline I & $\begin{array}{l}\text { Miscellaneous Engineers, Including Nuclear } \\
\text { Engineers }\end{array}$ & 1530 & 1510 & $\mathrm{x}$ & 0 & 0 & 3 \\
\hline I & $\begin{array}{l}\text { Miscellaneous Mathematical Science } \\
\text { Occupations, Including Mathematicians And }\end{array}$ & & & & & & \\
\hline \multirow[t]{2}{*}{ I } & $\begin{array}{l}\text { Statisticians } \\
\text { Miscellaneous Mathematical Science } \\
\text { Occupations, Including Mathematicians And }\end{array}$ & 1240 & 1210 & $\mathrm{x}$ & 0 & 2 & 1 \\
\hline & Statisticians & 1240 & 1230 & $\mathrm{x}$ & 2 & 3 & 0 \\
\hline $\mathrm{I}$ & Natural Sciences Managers & 360 & 360 & & 1 & 1 & 3 \\
\hline I & Operations Research Analysts & 1220 & 1220 & & 9 & 13 & 7 \\
\hline I & Optometrists & 3040 & 3040 & & 3 & 1 & 1 \\
\hline I & $\begin{array}{l}\text { Petroleum, Mining And Geological Engineers, } \\
\text { Including Mining Safety Engineers }\end{array}$ & 1520 & 1520 & & 0 & 3 & 0 \\
\hline I & $\begin{array}{l}\text { Petroleum, Mining And Geological Engineers, } \\
\text { Including Mining Safety Engineers }\end{array}$ & 1520 & 1500 & $\mathrm{x}$ & 1 & 0 & 1 \\
\hline I & Pharmacists & 3050 & 3050 & & 12 & 11 & 12 \\
\hline I & Physical Scientists, All Other & 1760 & 1760 & & 3 & 5 & 9 \\
\hline I & Physicians And Surgeons & 3060 & 3060 & & 38 & 43 & 33 \\
\hline $\mathrm{I}$ & Podiatrists & 3120 & 3120 & & 2 & 1 & 0 \\
\hline I & Postsecondary Teachers & 2200 & 2200 & & 98 & 117 & 105 \\
\hline $\mathrm{I}$ & Psychologists & 1820 & 1820 & & 15 & 13 & 15 \\
\hline I & $\begin{array}{l}\text { Software Developers, Applications And } \\
\text { Systems Software }\end{array}$ & 1020 & 1020 & & 44 & 74 & 61 \\
\hline I & Urban And Regional Planners & 1840 & 1840 & & 3 & 7 & 1 \\
\hline I & Veterinarians & 3250 & 3250 & & 5 & 4 & 3 \\
\hline II & Advertising And Promotions Managers & 40 & 40 & & 12 & 4 & 2 \\
\hline II & $\begin{array}{l}\text { Agents And Business Managers Of Artists, } \\
\text { Performers, And Athletes }\end{array}$ & 500 & 500 & & 7 & 5 & 4 \\
\hline II & Aircraft Pilots And Flight Engineers & 9030 & 9030 & & 17 & 13 & 7 \\
\hline \multirow[t]{2}{*}{ II } & Archivists, Curators, And Museum & & & & & & \\
\hline & Technicians & 2400 & 2400 & & 2 & 8 & 6 \\
\hline II & Business Operations Specialists, All Other & 740 & 740 & & 17 & 30 & 24 \\
\hline II & Clergy & 2040 & 2040 & & 28 & 28 & 20 \\
\hline II & Compensation And Benefits Managers & 135 & 135 & & 2 & 5 & 4 \\
\hline \multirow[t]{2}{*}{ II } & Compensation, Benefits, And Job Analysis & & & & & & \\
\hline & Specialists & 640 & 640 & & 4 & 4 & 7 \\
\hline II & Compliance Officers & 565 & 565 & & 10 & 6 & 16 \\
\hline II & Computer Network Architects & 1106 & 1106 & & 2 & 12 & 5 \\
\hline II & Computer Occupations, All Other & 1107 & 1107 & & 6 & 8 & 13 \\
\hline II & Computer Programmers & 1010 & 1010 & & 53 & 45 & 17 \\
\hline II & Computer Systems Analysts & 1006 & 1006 & & 36 & 35 & 34 \\
\hline
\end{tabular}




\begin{tabular}{|c|c|c|c|c|c|c|c|}
\hline Class & Occupational Title (ACS 2012) & $\begin{array}{l}\text { ACS } \\
2012\end{array}$ & $\begin{array}{r}\text { GSS } \\
\text { occ10 }\end{array}$ & $\begin{array}{l}\text { Recoded } \\
\text { to ACS }\end{array}$ & $\begin{array}{r}\text { GSS } \\
1994- \\
2000 \\
\end{array}$ & $\begin{array}{r}\text { GSS } \\
2002- \\
2008 \\
\end{array}$ & $\begin{array}{r}\text { GSS } \\
2010- \\
2016 \\
\end{array}$ \\
\hline II & Counselors & 2000 & 2000 & & 46 & 66 & 39 \\
\hline II & Database Administrators & 1060 & 1060 & & 8 & 10 & 3 \\
\hline II & Dietitians And Nutritionists & 3030 & 3030 & & 9 & 9 & 7 \\
\hline II & Directors, Religious Activities And Education & 2050 & 2050 & & 1 & 6 & 1 \\
\hline II & Editors & 2830 & 2830 & & 12 & 16 & 16 \\
\hline II & Elementary And Middle School Teachers & 2310 & 2310 & & 205 & 254 & 193 \\
\hline II & Emergency Management Directors & 425 & 425 & & 1 & 1 & 0 \\
\hline II & Financial Examiners & 900 & 900 & & 2 & 1 & 1 \\
\hline II & Financial Managers & 120 & 120 & & 64 & 76 & 66 \\
\hline II & Financial Specialists, All Other & 950 & 950 & & 3 & 3 & 4 \\
\hline II & Fundraisers & 726 & 726 & & 4 & 6 & 2 \\
\hline II & General And Operations Managers & 20 & 20 & & 53 & 56 & 74 \\
\hline II & $\begin{array}{l}\text { Health Diagnosing And Treating Practitioners, } \\
\text { All Other }\end{array}$ & 3260 & 3260 & & 1 & 3 & 2 \\
\hline II & Human Resources Managers & 136 & 136 & & 17 & 16 & 16 \\
\hline II & Industrial Production Managers & 140 & 140 & & 31 & 24 & 20 \\
\hline II & Information Security Analysts & 1007 & 1007 & & 2 & 5 & 3 \\
\hline II & Insurance Underwriters & 860 & 860 & & 4 & 7 & 8 \\
\hline II & Librarians & 2430 & 2430 & & 19 & 19 & 25 \\
\hline II & Logisticians & 700 & 700 & & 1 & 3 & 10 \\
\hline II & $\begin{array}{l}\text { Market Research Analysts And Marketing } \\
\text { Specialists }\end{array}$ & 735 & 735 & & 13 & 12 & 12 \\
\hline II & Marketing And Sales Managers & 50 & 50 & & 76 & 78 & 51 \\
\hline II & $\begin{array}{l}\text { Miscellaneous Community And Social Service } \\
\text { Specialists, Including Health Educators And }\end{array}$ & & & & & & \\
\hline II & $\begin{array}{l}\text { Community Health Workers } \\
\text { Miscellaneous Managers, Including Funeral } \\
\text { Service Managers And Postmasters And Mail }\end{array}$ & 2025 & 2025 & & 2 & 12 & 9 \\
\hline II & $\begin{array}{l}\text { Superintendents } \\
\text { Miscellaneous Managers, Including Funeral } \\
\text { Service Managers And Postmasters And Mail }\end{array}$ & 430 & 430 & & 162 & 204 & 146 \\
\hline \multirow[t]{2}{*}{ II } & $\begin{array}{l}\text { Superintendents } \\
\text { Miscellaneous Managers, Including Funeral } \\
\text { Service Managers And Postmasters And Mail }\end{array}$ & 430 & 400 & $x$ & 1 & 7 & 1 \\
\hline & Superintendents & 430 & 325 & $x$ & 0 & 4 & 0 \\
\hline \multirow[t]{2}{*}{ II } & Miscellaneous Social Scientists, Including & & & & & & \\
\hline & Survey Researchers And Sociologists & 1860 & 1860 & & 4 & 7 & 5 \\
\hline II & Miscellaneous Social Scientists, Including & & & & & & \\
\hline \multirow[t]{2}{*}{ II } & $\begin{array}{l}\text { Survey Researchers And Sociologists } \\
\text { Miscellaneous Social Scientists, Including }\end{array}$ & 1860 & 1815 & $x$ & 0 & 1 & 0 \\
\hline & $\begin{array}{l}\text { Survey Researchers And Sociologists } \\
\text { Network And Computer Systems }\end{array}$ & 1860 & 1830 & $x$ & 0 & 1 & 0 \\
\hline II & Administrators & 1105 & 1105 & & 9 & 24 & 17 \\
\hline \multirow[t]{2}{*}{ II } & News Analysts, Reporters And & & & & & & \\
\hline & Correspondents & 2810 & 2810 & & 11 & 7 & 5 \\
\hline II & Nurse Anesthetists & 3256 & 3256 & & 0 & 2 & 3 \\
\hline II & Nurse Practitioners, And Nurse Midwives & 3258 & 3258 & & 3 & 5 & 5 \\
\hline
\end{tabular}




\begin{tabular}{|c|c|c|c|c|c|c|c|}
\hline Class & Occupational Title (ACS 2012) & $\begin{array}{l}\text { ACS } \\
2012 \\
\end{array}$ & $\begin{array}{r}\text { GSS } \\
\text { occ10 }\end{array}$ & $\begin{array}{l}\text { Recoded } \\
\text { to ACS }\end{array}$ & $\begin{array}{r}\text { GSS } \\
1994- \\
2000 \\
\end{array}$ & $\begin{array}{r}\text { GSS } \\
2002- \\
2008 \\
\end{array}$ & $\begin{array}{r}\text { GSS } \\
2010- \\
2016 \\
\end{array}$ \\
\hline II & Occupational Therapists & 3150 & 3150 & & 4 & 5 & 5 \\
\hline II & Other Education, Training, And Library & & & & & & \\
\hline II & $\begin{array}{l}\text { Workers } \\
\text { Other Healthcare Practitioners And Technical }\end{array}$ & 2550 & 2550 & & 6 & 6 & 8 \\
\hline II & $\begin{array}{l}\text { Occupations } \\
\text { Other Therapists, Including Exercise }\end{array}$ & 3540 & 3540 & & 8 & 9 & 6 \\
\hline II & $\begin{array}{l}\text { Physiologists } \\
\text { Other Therapists, Including Exercise }\end{array}$ & 3245 & 3245 & & 8 & 11 & 8 \\
\hline & Physiologists & 3245 & 3235 & $\mathrm{x}$ & 1 & 0 & 0 \\
\hline II & Personal Financial Advisors & 850 & 850 & & 17 & 24 & 18 \\
\hline II & Physical Therapists & 3160 & 3160 & & 13 & 11 & 7 \\
\hline II & Physician Assistants & 3110 & 3110 & & 0 & 5 & 5 \\
\hline II & Producers And Directors & 2710 & 2710 & & 10 & 21 & 15 \\
\hline II & Public Relations And Fundraising Managers & 60 & 60 & & 5 & 4 & 8 \\
\hline II & Public Relations Specialists & 2825 & 2825 & & 14 & 16 & 6 \\
\hline II & $\begin{array}{l}\text { Purchasing Agents, Except Wholesale, Retail, } \\
\text { And Farm Products }\end{array}$ & 530 & 530 & & 14 & 25 & 14 \\
\hline II & Purchasing Managers & 150 & 150 & & 15 & 7 & 15 \\
\hline II & Recreational Therapists & 3210 & 3210 & & 0 & 2 & 3 \\
\hline II & Registered Nurses & 3255 & 3255 & & 184 & 143 & 157 \\
\hline II & Sales Engineers & 4930 & 4930 & & 3 & 2 & 1 \\
\hline II & Secondary School Teachers & 2320 & 2320 & & 129 & 138 & 108 \\
\hline II & $\begin{array}{l}\text { Securities, Commodities, And Financial } \\
\text { Services Sales Agents }\end{array}$ & 4820 & 4820 & & 21 & 24 & 9 \\
\hline II & Social And Community Service Managers & 420 & 420 & & 20 & 22 & 26 \\
\hline II & Social Workers & 2010 & 2010 & & 65 & 86 & 58 \\
\hline II & Special Education Teachers & 2330 & 2330 & & 32 & 29 & 31 \\
\hline II & Speech-Language Pathologists & 3230 & 3230 & & 5 & 11 & 9 \\
\hline II & Surveyors, Cartographers, And & & & & & & \\
\hline & Photogrammetrists & 1310 & 1310 & & 3 & 4 & 4 \\
\hline II & Technical Writers & 2840 & 2840 & & 9 & 3 & 3 \\
\hline II & Training And Development Managers & 137 & 137 & & 5 & 5 & 3 \\
\hline II & Training And Development Specialists & 650 & 650 & & 5 & 14 & 8 \\
\hline II & Writers And Authors & 2850 & 2850 & & 12 & 18 & 13 \\
\hline IIIa & Administrative Services Managers & 100 & 100 & & 10 & 7 & 5 \\
\hline IIIa & Advertising Sales Agents & 4800 & 4800 & & 15 & 18 & 10 \\
\hline IIIa & $\begin{array}{l}\text { Air Traffic Controllers And Airfield } \\
\text { Operations Specialists }\end{array}$ & 9040 & 9040 & & 2 & 6 & 4 \\
\hline IIIa & Appraisers And Assessors Of Real Estate & 810 & 810 & & 10 & 6 & 7 \\
\hline IIIa & Bill And Account Collectors & 5100 & 5100 & & 10 & 13 & 11 \\
\hline IIIa & Billing And Posting Clerks & 5110 & 5110 & & 34 & 41 & 27 \\
\hline IIIIa & $\begin{array}{l}\text { Bookkeeping, Accounting, And Auditing } \\
\text { Clerks }\end{array}$ & 5120 & 5120 & & 149 & 127 & 80 \\
\hline IIIa & Brokerage Clerks & 5200 & 5200 & & 2 & 0 & 2 \\
\hline IIIa & Cargo And Freight Agents & 5500 & 5500 & & 2 & 4 & 0 \\
\hline
\end{tabular}




\begin{tabular}{|c|c|c|c|c|c|c|c|}
\hline Class & Occupational Title (ACS 2012) & $\begin{array}{l}\text { ACS } \\
2012\end{array}$ & $\begin{array}{r}\text { GSS } \\
\text { occ10 }\end{array}$ & $\begin{array}{l}\text { Recoded } \\
\text { to ACS }\end{array}$ & $\begin{array}{r}\text { GSS } \\
1994- \\
2000\end{array}$ & $\begin{array}{r}\text { GSS } \\
2002- \\
2008\end{array}$ & $\begin{array}{r}\text { GSS } \\
2010- \\
2016\end{array}$ \\
\hline IIIa & $\begin{array}{l}\text { Claims Adjusters, Appraisers, Examiners, And } \\
\text { Investigators }\end{array}$ & 540 & 540 & & 19 & 18 & 23 \\
\hline \multirow[t]{2}{*}{ IIIa } & Communications Equipment Operators, All & & & & & & \\
\hline & Other & 5030 & 5030 & & 0 & 1 & 1 \\
\hline IIIa & Computer Operators & 5800 & 5800 & & 16 & 14 & 9 \\
\hline IIIa & Computer Support Specialists & 1050 & 1050 & & 23 & 46 & 34 \\
\hline IIIa & Correspondence Clerks And Order Clerks & 5350 & 5350 & & 8 & 8 & 2 \\
\hline IIIa & Correspondence Clerks And Order Clerks & 5350 & 5210 & $x$ & 0 & 1 & 0 \\
\hline IIIa & Court, Municipal, And License Clerks & 5220 & 5220 & & 9 & 6 & 6 \\
\hline IIIa & Credit Analysts & 830 & 830 & & 5 & 5 & 1 \\
\hline IIIa & Credit Authorizers, Checkers, And Clerks & 5230 & 5230 & & 9 & 5 & 1 \\
\hline IIIa & Credit Counselors And Loan Officers & 910 & 910 & & 24 & 23 & 15 \\
\hline IIIa & Customer Service Representatives & 5240 & 5240 & & 98 & 141 & 151 \\
\hline IIIa & Data Entry Keyers & 5810 & 5810 & & 38 & 45 & 14 \\
\hline IIIa & Dispatchers & 5520 & 5520 & & 15 & 31 & 11 \\
\hline \multirow[t]{2}{*}{ IIIa } & Eligibility Interviewers, Government & & & & & & \\
\hline & Programs & 5250 & 5250 & & 5 & 5 & 3 \\
\hline IIIa & File Clerks & 5260 & 5260 & & 11 & 12 & 6 \\
\hline IIIa & Financial Clerks, All Other & 5165 & 5165 & & 2 & 0 & 5 \\
\hline \multirow[t]{2}{*}{ IIIa } & First-Line Supervisors Of Office And & & & & & & \\
\hline & Administrative Support Workers & 5000 & 5000 & & 143 & 139 & 122 \\
\hline IIIa & Flight Attendants & 9050 & 9050 & & 8 & 4 & 7 \\
\hline IIIa & Hotel, Motel, And Resort Desk Clerks & 5300 & 5300 & & 5 & 13 & 9 \\
\hline \multirow[t]{2}{*}{ IIIa } & Human Resources Assistants, Except Payroll & & & & & & \\
\hline & And Timekeeping & 5360 & 5360 & & 11 & 10 & 11 \\
\hline IIIa & Human Resources Workers & 630 & 630 & & 27 & 34 & 42 \\
\hline IIIa & Information And Record Clerks, All Other & 5420 & 5420 & & 8 & 13 & 3 \\
\hline \multirow[t]{2}{*}{ IIIa } & Insurance Claims And Policy Processing & & & & & & \\
\hline & Clerks & 5840 & 5840 & & 28 & 15 & 18 \\
\hline IIIa & Insurance Sales Agents & 4810 & 4810 & & 29 & 46 & 38 \\
\hline IIIa & Interviewers, Except Eligibility And Loan & 5310 & 5310 & & 13 & 18 & 12 \\
\hline IIIa & Library Assistants, Clerical & 5320 & 5320 & & 20 & 12 & 3 \\
\hline IIIa & Library Technicians & 2440 & 2440 & & 2 & 3 & 3 \\
\hline \multirow[t]{2}{*}{ IIIa } & Licensed Practical And Licensed Vocational & & & & & & \\
\hline & Nurses & 3500 & 3500 & & 60 & 40 & 26 \\
\hline IIIa & Loan Interviewers And Clerks & 5330 & 5330 & & 18 & 12 & 9 \\
\hline IIIa & Lodging Managers & 340 & 340 & & 8 & 15 & 10 \\
\hline \multirow[t]{2}{*}{ IIIa } & Medical Records And Health Information & & & & & & \\
\hline & Technicians & 3510 & 3510 & & 6 & 5 & 10 \\
\hline IIIa & Meeting, Convention, And Event Planners & 725 & 725 & & 9 & 7 & 2 \\
\hline IIIa & Meter Readers, Utilities & 5530 & 5530 & & 3 & 5 & 0 \\
\hline IIIa & Miscellaneous Legal Support Workers & 2160 & 2160 & & 15 & 15 & 17 \\
\hline IIIa & $\begin{array}{l}\text { Miscellaneous Office And Administrative } \\
\text { Support Workers, Including Desktop }\end{array}$ & & & & & & \\
\hline & Publishers & 5940 & 5940 & & 48 & 45 & 41 \\
\hline
\end{tabular}




\begin{tabular}{|c|c|c|c|c|c|c|c|}
\hline Class & Occupational Title (ACS 2012) & $\begin{array}{l}\text { ACS } \\
2012\end{array}$ & $\begin{array}{r}\text { GSS } \\
\text { occ10 }\end{array}$ & $\begin{array}{l}\text { Recoded } \\
\text { to ACS }\end{array}$ & $\begin{array}{r}\text { GSS } \\
1994- \\
2000\end{array}$ & $\begin{array}{r}\text { GSS } \\
2002- \\
2008\end{array}$ & $\begin{array}{r}\text { GSS } \\
2010- \\
2016\end{array}$ \\
\hline \multirow[t]{2}{*}{ IIIa } & $\begin{array}{l}\text { Miscellaneous Office And Administrative } \\
\text { Support Workers, Including Desktop }\end{array}$ & & & & & & \\
\hline & Publishers & 5940 & 5830 & $x$ & 1 & 1 & 0 \\
\hline IIIa & New Accounts Clerks & 5340 & 5340 & & 1 & 1 & 0 \\
\hline IIIa & Office Clerks, General & 5860 & 5860 & & 97 & 95 & 92 \\
\hline IIIa & Office Machine Operators, Except Computer & 5900 & 5900 & & 5 & 4 & 1 \\
\hline IIIa & Other Teachers And Instructors & 2340 & 2340 & & 47 & 45 & 47 \\
\hline IIIa & Paralegals And Legal Assistants & 2145 & 2145 & & 27 & 28 & 25 \\
\hline IIIa & Payroll And Timekeeping Clerks & 5140 & 5140 & & 23 & 24 & 9 \\
\hline IIIa & Postal Service Clerks & 5540 & 5540 & & 25 & 21 & 9 \\
\hline IIIa & Procurement Clerks & 5150 & 5150 & & 4 & 1 & 1 \\
\hline IIIa & Production, Planning, And Expediting Clerks & 5600 & 5600 & & 14 & 27 & 19 \\
\hline IIIa & Proofreaders And Copy Markers & 5910 & 5910 & & 1 & 1 & 0 \\
\hline IIIa & Radiation Therapists & 3200 & 3200 & & 0 & 1 & 1 \\
\hline IIIa & Real Estate Brokers And Sales Agents & 4920 & 4920 & & 68 & 76 & 51 \\
\hline IIIa & $\begin{array}{l}\text { Reservation And Transportation Ticket Agents } \\
\text { And Travel Clerks }\end{array}$ & 5410 & 5410 & & 16 & 14 & 6 \\
\hline IIIa & Respiratory Therapists & 3220 & 3220 & & 7 & 5 & 4 \\
\hline IIIa & Sales And Related Workers, All Other & 4965 & 4965 & & 15 & 41 & 27 \\
\hline IIIa & Sales Representatives, Services, All Other & 4840 & 4840 & & 26 & 19 & 20 \\
\hline IIIa & $\begin{array}{l}\text { Sales Representatives, Wholesale And } \\
\text { Manufacturing }\end{array}$ & 4850 & 4850 & & 88 & 129 & 60 \\
\hline IIIa & Secretaries And Administrative Assistants & 5700 & 5700 & & 462 & 402 & 232 \\
\hline IIIa & Social And Human Service Assistants & 2016 & 2016 & & 9 & 11 & 11 \\
\hline IIIa & Statistical Assistants & 5920 & 5920 & & 5 & 2 & 4 \\
\hline IIIa & $\begin{array}{l}\text { Switchboard Operators, Including Answering } \\
\text { Service }\end{array}$ & 5010 & 5010 & & 19 & 11 & 7 \\
\hline \multirow[t]{2}{*}{ IIIa } & Tax Examiners And Collectors, And Revenue & & & & & & \\
\hline & Agents & 930 & 930 & & 6 & 3 & 8 \\
\hline IIIa & Tax Preparers & 940 & 940 & & 8 & 8 & 12 \\
\hline IIIa & Teacher Assistants & 2540 & 2540 & & 69 & 76 & 61 \\
\hline IIIa & Telephone Operators & 5020 & 5020 & & 20 & 12 & 7 \\
\hline IIIa & Tellers & 5160 & 5160 & & 37 & 40 & 23 \\
\hline IIIa & Tour And Travel Guides & 4540 & 4540 & & 2 & 1 & 2 \\
\hline IIIa & Travel Agents & 4830 & 4830 & & 17 & 8 & 7 \\
\hline IIIa & Web Developers & 1030 & 1030 & & 2 & 13 & 10 \\
\hline \multirow[t]{2}{*}{ IIIa } & Weighers, Measurers, Checkers, And & & & & & & \\
\hline & Samplers, Recordkeeping & 5630 & 5630 & & 11 & 16 & 3 \\
\hline IIIa & Word Processors And Typists & 5820 & 5820 & & 33 & 29 & 9 \\
\hline $\mathrm{IIIb}$ & Animal Control Workers & 3900 & 3900 & & 1 & 1 & 0 \\
\hline \multirow[t]{2}{*}{$\mathrm{IIIb}$} & Automotive And Watercraft Service & & & & & & \\
\hline & Attendants & 9360 & 9360 & & 9 & 7 & 4 \\
\hline $\mathrm{IIIb}$ & Baggage Porters, Bellhops, And Concierges & 4530 & 4530 & & 9 & 9 & 13 \\
\hline $\mathrm{IIIb}$ & Barbers & 4500 & 4500 & & 3 & 7 & 7 \\
\hline $\mathrm{IIIb}$ & Bartenders & 4040 & 4040 & & 34 & 36 & 33 \\
\hline
\end{tabular}




\begin{tabular}{|c|c|c|c|c|c|c|c|}
\hline Class & Occupational Title (ACS 2012) & $\begin{array}{l}\text { ACS } \\
2012 \\
\end{array}$ & $\begin{array}{r}\text { GSS } \\
\text { occ10 }\end{array}$ & $\begin{array}{l}\text { Recoded } \\
\text { to ACS }\end{array}$ & $\begin{array}{r}\text { GSS } \\
1994- \\
2000\end{array}$ & $\begin{array}{r}\text { GSS } \\
2002- \\
2008\end{array}$ & $\begin{array}{r}\text { GSS } \\
2010- \\
2016\end{array}$ \\
\hline IIIb & Bus Drivers & 9120 & 9120 & & 45 & 42 & 37 \\
\hline IIIb & Cashiers & 4720 & 4720 & & 188 & 187 & 153 \\
\hline IIIb & Childcare Workers & 4600 & 4600 & & 125 & 129 & 105 \\
\hline $\mathrm{IIIb}$ & $\begin{array}{l}\text { Coin, Vending, And Amusement Machine } \\
\text { Servicers And Repairers } \\
\text { Combined Food Preparation And Serving }\end{array}$ & 7510 & 7510 & & 2 & 3 & 3 \\
\hline IIIb & $\begin{array}{l}\text { Workers, Including Fast Food } \\
\text { Counter And Rental Clerks }\end{array}$ & $\begin{array}{l}4050 \\
4740\end{array}$ & $\begin{array}{l}4050 \\
4740\end{array}$ & & $\begin{array}{l}32 \\
17\end{array}$ & $\begin{array}{r}40 \\
9\end{array}$ & $\begin{array}{l}55 \\
12\end{array}$ \\
\hline $\mathrm{IIIb}$ & $\begin{array}{l}\text { Counter Attendants, Cafeteria, Food } \\
\text { Concession, And Coffee Shop }\end{array}$ & 4060 & 4060 & & 14 & 10 & 13 \\
\hline IIIb & Couriers And Messengers & 5510 & 5510 & & 12 & 24 & 17 \\
\hline IIIb & Dental Assistants & 3640 & 3640 & & 12 & 19 & 23 \\
\hline IIIlb & $\begin{array}{l}\text { Door-To-Door Sales Workers, News And } \\
\text { Street Vendors, And Related Workers }\end{array}$ & 4950 & 4950 & & 18 & 12 & 8 \\
\hline IIIb & Embalmers And Funeral Attendants & 4460 & 4460 & & 0 & 2 & 0 \\
\hline IIIb & First-Line Supervisors Of Gaming Workers & 4300 & 4300 & & 3 & 0 & 2 \\
\hline IIIlb & $\begin{array}{l}\text { First-Line Supervisors Of Housekeeping And } \\
\text { Janitorial Workers }\end{array}$ & 4200 & 4200 & & 23 & 22 & 16 \\
\hline $\mathrm{IIIb}$ & $\begin{array}{l}\text { First-Line Supervisors Of Personal Service } \\
\text { Workers }\end{array}$ & 4320 & 4320 & & 13 & 9 & 5 \\
\hline IIIb & First-Line Supervisors Of Retail Sales Workers & 4700 & 4700 & & 233 & 216 & 167 \\
\hline IIIb & $\begin{array}{l}\text { First-Line Supervisors Of Food Preparation } \\
\text { And Serving Workers }\end{array}$ & 4010 & 4010 & & 58 & 61 & 45 \\
\hline $\mathrm{IIIb}$ & Food Servers, Nonrestaurant & 4120 & 4120 & & 12 & 7 & 10 \\
\hline $\mathrm{IIIb}$ & Food Service Managers & 310 & 310 & & 76 & 54 & 40 \\
\hline IIIb & Gaming Cage Workers & 5130 & 5130 & & 1 & 0 & 0 \\
\hline $\mathrm{IIIb}$ & Gaming Services Workers & 4400 & 4400 & & 7 & 7 & 2 \\
\hline $\mathrm{IIIb}$ & Hairdressers, Hairstylists, And Cosmetologists & 4510 & 4510 & & 82 & 70 & 67 \\
\hline $\mathrm{IIIb}$ & $\begin{array}{l}\text { Healthcare Support Workers, All Other, } \\
\text { Including Medical Equipment Preparers }\end{array}$ & 3655 & 3655 & & 16 & 24 & 15 \\
\hline IIIb & $\begin{array}{l}\text { Hosts And Hostesses, Restaurant, Lounge, } \\
\text { And Coffee Shop }\end{array}$ & 4150 & 4150 & & 16 & 3 & 11 \\
\hline IIIb & $\begin{array}{l}\text { Lifeguards And Other Recreational, And All } \\
\text { Other Protective Service Workers }\end{array}$ & 3955 & 3955 & & 8 & 6 & 4 \\
\hline IIIb & Massage Therapists & 3630 & 3630 & & 6 & 13 & 17 \\
\hline IIIb & Medical Assistants & 3645 & 3645 & & 26 & 26 & 23 \\
\hline IIIlb & Medical Transcriptionists & 3646 & 3646 & & 7 & 9 & 2 \\
\hline $\mathrm{IIIb}$ & $\begin{array}{l}\text { Miscellaneous Entertainment Attendants And } \\
\text { Related Workers }\end{array}$ & 4430 & 4430 & & 13 & 10 & 16 \\
\hline IIIb & $\begin{array}{l}\text { Miscellaneous Food Preparation And Serving } \\
\text { Related Workers, Including Dining Room And }\end{array}$ & & & & & & \\
\hline IIIb & $\begin{array}{l}\text { Cafeteria Attendants And Bartender Helpers } \\
\text { Miscellaneous Food Preparation And Serving } \\
\text { Related Workers, Including Dining Room And }\end{array}$ & 4130 & 4130 & & 15 & 26 & 21 \\
\hline IIIlb & $\begin{array}{l}\text { Cafeteria Attendants And Bartender Helpers } \\
\text { Miscellaneous Personal Appearance Workers }\end{array}$ & $\begin{array}{l}4130 \\
4520\end{array}$ & $\begin{array}{l}4160 \\
4520\end{array}$ & $\mathrm{x}$ & $\begin{array}{l}0 \\
3\end{array}$ & $\begin{array}{r}2 \\
10\end{array}$ & $\begin{array}{r}2 \\
11\end{array}$ \\
\hline
\end{tabular}




\begin{tabular}{|c|c|c|c|c|c|c|c|}
\hline Class & Occupational Title (ACS 2012) & $\begin{array}{l}\text { ACS } \\
2012\end{array}$ & $\begin{array}{r}\text { GSS } \\
\text { occ10 }\end{array}$ & $\begin{array}{l}\text { Recoded } \\
\text { to ACS }\end{array}$ & $\begin{array}{r}\text { GSS } \\
1994- \\
2000\end{array}$ & $\begin{array}{r}\text { GSS } \\
2002- \\
2008\end{array}$ & $\begin{array}{r}\text { GSS } \\
2010- \\
2016\end{array}$ \\
\hline $\mathrm{IIIb}$ & $\begin{array}{l}\text { Miscellaneous Transportation Workers, } \\
\text { Including Bridge And Lock Tenders And } \\
\text { Traffic Technicians }\end{array}$ & 9420 & 9420 & & 1 & 2 & 0 \\
\hline \multirow[t]{2}{*}{$\mathrm{IIIb}$} & Models, Demonstrators, And Product & & & & & & \\
\hline & Promoters & 4900 & 4900 & & 6 & 8 & 4 \\
\hline \multirow[t]{2}{*}{$\mathrm{IIIb}$} & Morticians, Undertakers, And Funeral & & & & & & \\
\hline & Directors & 4465 & 4465 & & 1 & 1 & 4 \\
\hline IIIb & Motion Picture Projectionists & 4410 & 4410 & & 0 & 1 & 3 \\
\hline $\mathrm{IIIb}$ & Nonfarm Animal Caretakers & 4350 & 4350 & & 9 & 11 & 8 \\
\hline $\mathrm{IIIb}$ & Nursing, Psychiatric, And Home Health Aides & 3600 & 3600 & & 233 & 274 & 230 \\
\hline IIIb & Occupational Therapy Assistants And Aides & 3610 & 3610 & & 1 & 3 & 0 \\
\hline $\mathrm{IIIb}$ & Parking Lot Attendants & 9350 & 9350 & & 7 & 1 & 5 \\
\hline $\mathrm{IIIb}$ & Parts Salespersons & 4750 & 4750 & & 11 & 10 & 15 \\
\hline $\mathrm{IIIb}$ & Personal Care Aides & 4610 & 4610 & & 43 & 46 & 71 \\
\hline IIIb & Personal Care And Service Workers, All Other & 4650 & 4650 & & 1 & 5 & 9 \\
\hline $\mathrm{IIIb}$ & Pest Control Workers & 4240 & 4240 & & 5 & 3 & 4 \\
\hline $\mathrm{IIIb}$ & Pharmacy Aides & 3647 & 3647 & & 1 & 9 & 2 \\
\hline $\mathrm{IIIb}$ & Phlebotomists & 3649 & 3649 & & 3 & 6 & 12 \\
\hline $\mathrm{IIIb}$ & Physical Therapist Assistants And Aides & 3620 & 3620 & & 6 & 7 & 4 \\
\hline IIIb & Preschool And Kindergarten Teachers & 2300 & 2300 & & 34 & 51 & 53 \\
\hline $\mathrm{IIIb}$ & Receptionists And Information Clerks & 5400 & 5400 & & 88 & 69 & 54 \\
\hline $\mathrm{IIIb}$ & Recreation And Fitness Workers & 4620 & 4620 & & 10 & 30 & 24 \\
\hline $\mathrm{IIIb}$ & Religious Workers, All Other & 2060 & 2060 & & 1 & 6 & 6 \\
\hline $\mathrm{IIIb}$ & Residential Advisors & 4640 & 4640 & & 4 & 4 & 0 \\
\hline $\mathrm{IIIb}$ & Retail Salespersons & 4760 & 4760 & & 262 & 249 & 191 \\
\hline IIIb & $\begin{array}{l}\text { Security Guards And Gaming Surveillance } \\
\text { Officers }\end{array}$ & 3930 & 3930 & & 43 & 50 & 31 \\
\hline $\mathrm{IIIb}$ & Telemarketers & 4940 & 4940 & & 20 & 14 & 8 \\
\hline \multirow[t]{2}{*}{$\mathrm{IIIb}$} & Transportation Attendants, Except Flight & & & & & & \\
\hline & Attendants & 9415 & 9415 & & 1 & 1 & 2 \\
\hline $\mathrm{IIIb}$ & Transportation Security Screeners & 3945 & 3945 & & 1 & 4 & 0 \\
\hline $\mathrm{IIIb}$ & Ushers, Lobby Attendants, And Ticket Takers & 4420 & 4420 & & 3 & 1 & 2 \\
\hline $\mathrm{IIIb}$ & $\begin{array}{l}\text { Veterinary Assistants And Laboratory Animal } \\
\text { Caretakers }\end{array}$ & 3648 & 3648 & & 5 & 2 & 2 \\
\hline IIIb & Waiters And Waitresses & 4110 & 4110 & & 179 & 187 & 132 \\
\hline \multirow[t]{2}{*}{$\mathrm{IIIb}$} & Wholesale And Retail Buyers, Except Farm & & & & & & \\
\hline & Products & 520 & 520 & & 17 & 15 & 6 \\
\hline \multirow[t]{2}{*}{ IVc } & Farmers, Ranchers, And Other Agricultural & & & & & & \\
\hline & Managers & 205 & 205 & & 69 & 63 & 25 \\
\hline $\mathrm{V}$ & Actors & 2700 & 2700 & & 4 & 2 & 1 \\
\hline $\mathrm{V}$ & Agricultural And Food Science Technicians & 1900 & 1900 & & 5 & 3 & 5 \\
\hline $\mathrm{V}$ & Announcers & 2800 & 2800 & & 4 & 2 & 5 \\
\hline $\mathrm{V}$ & Artists And Related Workers & 2600 & 2600 & & 28 & 31 & 14 \\
\hline \multirow[t]{2}{*}{$\mathrm{V}$} & Athletes, Coaches, Umpires, And Related & & & & & & \\
\hline & Workers & 2720 & 2720 & & 9 & 11 & 16 \\
\hline V & Avionics Technicians & 7030 & 7030 & & 3 & 1 & 1 \\
\hline
\end{tabular}




\begin{tabular}{|c|c|c|c|c|c|c|c|}
\hline Class & Occupational Title (ACS 2012) & $\begin{array}{l}\text { ACS } \\
2012 \\
\end{array}$ & $\begin{array}{r}\text { GSS } \\
\text { occ10 }\end{array}$ & $\begin{array}{l}\text { Recoded } \\
\text { to ACS }\end{array}$ & $\begin{array}{r}\text { GSS } \\
1994- \\
2000 \\
\end{array}$ & $\begin{array}{r}\text { GSS } \\
2002- \\
2008 \\
\end{array}$ & $\begin{array}{r}\text { GSS } \\
2010- \\
2016 \\
\end{array}$ \\
\hline $\mathrm{V}$ & Bailiffs, Correctional Officers, And Jailers & 3800 & 3800 & & 22 & 24 & 28 \\
\hline $\mathrm{V}$ & Biological Technicians & 1910 & 1910 & & 1 & 3 & 3 \\
\hline \multirow[t]{2}{*}{$\mathrm{V}$} & $\begin{array}{l}\text { Broadcast And Sound Engineering } \\
\text { Technicians And Radio Operators, And Media } \\
\text { And Communication Equipment Workers, All }\end{array}$ & & & & & & \\
\hline & Other & 2900 & 2900 & & 4 & 7 & 9 \\
\hline \multirow[t]{2}{*}{$\mathrm{V}$} & Buyers And Purchasing Agents, Farm & & & & & & \\
\hline & Products & 510 & 510 & & 3 & 4 & 2 \\
\hline $\mathrm{V}$ & Chefs And Head Cooks & 4000 & 4000 & & 15 & 25 & 28 \\
\hline $\mathrm{V}$ & Chemical Technicians & 1920 & 1920 & & 11 & 6 & 6 \\
\hline \multirow[t]{2}{*}{$\mathrm{V}$} & Clinical Laboratory Technologists And & & & & & & \\
\hline & $\begin{array}{l}\text { Technicians } \\
\text { Computer Control Programmers And }\end{array}$ & 3300 & 3300 & & 21 & 27 & 26 \\
\hline $\mathrm{V}$ & Operators & 7900 & 7900 & & 2 & 0 & 1 \\
\hline $\mathrm{V}$ & Construction And Building Inspectors & 6660 & 6660 & & 6 & 5 & 3 \\
\hline $\mathrm{V}$ & Construction Managers & 220 & 220 & & 52 & 76 & 53 \\
\hline $\mathrm{V}$ & Cost Estimators & 600 & 600 & & 6 & 3 & 3 \\
\hline $\mathrm{V}$ & Dancers And Choreographers & 2740 & 2740 & & 5 & 1 & 1 \\
\hline $\mathrm{V}$ & Dental Hygienists & 3310 & 3310 & & 9 & 7 & 5 \\
\hline $\mathrm{V}$ & Designers & 2630 & 2630 & & 51 & 64 & 43 \\
\hline $\mathrm{V}$ & Detectives And Criminal Investigators & 3820 & 3820 & & 9 & 12 & 10 \\
\hline \multirow[t]{2}{*}{$\mathrm{V}$} & Diagnostic Related Technologists And & & & & & & \\
\hline & Technicians & 3320 & 3320 & & 19 & 21 & 27 \\
\hline $\mathrm{V}$ & Drafters & 1540 & 1540 & & 13 & 20 & 6 \\
\hline \multirow[t]{2}{*}{$\mathrm{V}$} & Electric Motor, Power Tool, And Related & & & & & & \\
\hline & Repairers & 7040 & 7040 & & 8 & 4 & 5 \\
\hline \multirow[t]{2}{*}{$\mathrm{V}$} & $\begin{array}{l}\text { Electrical And Electronics Repairers, } \\
\text { Transportation Equipment, And Industrial }\end{array}$ & & & & & & \\
\hline & And Utility & 7100 & 7100 & & 2 & 2 & 0 \\
\hline \multirow[t]{2}{*}{$\mathrm{V}$} & Electronic Equipment Installers And & & & & & & \\
\hline & Repairers, Motor Vehicles & 7110 & 7110 & & 5 & 2 & 2 \\
\hline \multirow[t]{2}{*}{$\mathrm{V}$} & Electronic Home Entertainment Equipment & & & & & & \\
\hline & Installers And Repairers & 7120 & 7120 & & 5 & 3 & 3 \\
\hline $\mathrm{V}$ & Elevator Installers And Repairers & 6700 & 6700 & & 1 & 2 & 0 \\
\hline \multirow[t]{2}{*}{$\mathrm{V}$} & Emergency Medical Technicians And & & & & & & \\
\hline & Paramedics & 3400 & 3400 & & 7 & 14 & 10 \\
\hline $\mathrm{V}$ & Engineering Technicians, Except Drafters & 1550 & 1550 & & 54 & 38 & 25 \\
\hline \multirow[t]{2}{*}{$\mathrm{V}$} & Entertainers And Performers, Sports And & & & & & & \\
\hline & Related Workers, All Other & 2760 & 2760 & & 3 & 5 & 2 \\
\hline $\mathrm{V}$ & Fire Inspectors & 3750 & 3750 & & 1 & 5 & 1 \\
\hline $\mathrm{V}$ & Firefighters & 3740 & 3740 & & 15 & 29 & 16 \\
\hline \multirow[t]{2}{*}{$\mathrm{V}$} & First-Line Supervisors Of Construction Trades & & & & & & \\
\hline & And Extraction Workers & 6200 & 6200 & & 62 & 78 & 31 \\
\hline $\mathrm{V}$ & First-Line Supervisors Of Correctional Officers & 3700 & 3700 & & 5 & 8 & 5 \\
\hline $\mathrm{V}$ & $\begin{array}{l}\text { First-Line Supervisors Of Landscaping, Lawn } \\
\text { Service, And Groundskeeping Workers }\end{array}$ & 4210 & 4210 & & 14 & 14 & 15 \\
\hline
\end{tabular}




\begin{tabular}{|c|c|c|c|c|c|c|c|}
\hline Class & Occupational Title (ACS 2012) & $\begin{array}{l}\text { ACS } \\
2012\end{array}$ & $\begin{array}{r}\text { GSS } \\
\text { occ10 }\end{array}$ & $\begin{array}{l}\text { Recoded } \\
\text { to ACS }\end{array}$ & $\begin{array}{r}\text { GSS } \\
1994- \\
2000 \\
\end{array}$ & $\begin{array}{r}\text { GSS } \\
2002- \\
2008 \\
\end{array}$ & $\begin{array}{r}\text { GSS } \\
2010- \\
2016 \\
\end{array}$ \\
\hline \multirow[t]{2}{*}{$\mathrm{V}$} & First-Line Supervisors Of Mechanics, & & & & & & \\
\hline & Installers, And Repairers & 7000 & 7000 & & 33 & 44 & 13 \\
\hline \multirow[t]{2}{*}{ V } & First-Line Supervisors Of Non-Retail Sales & & & & & & \\
\hline & Workers & 4710 & 4710 & & 57 & 62 & 39 \\
\hline \multirow[t]{2}{*}{$\mathrm{V}$} & First-Line Supervisors Of Production And & & & & & & \\
\hline & Operating Workers & 7700 & 7700 & & 108 & 87 & 42 \\
\hline \multirow[t]{2}{*}{ V } & First-Line Supervisors Of Protective Service & & & & & & \\
\hline & Workers, All Other & 3730 & 3730 & & 10 & 11 & 6 \\
\hline \multirow[t]{2}{*}{$\mathrm{V}$} & First-Line Supervisors Of Fire Fighting And & & & & & & \\
\hline & Prevention Workers & 3720 & 3720 & & 4 & 3 & 2 \\
\hline \multirow[t]{2}{*}{$\mathrm{V}$} & First-Line Supervisors Of Police And & & & & & & \\
\hline & Detectives & 3710 & 3710 & & 7 & 3 & 8 \\
\hline $\mathrm{V}$ & Gaming Managers & 330 & 330 & & 2 & 3 & 3 \\
\hline \multirow[t]{2}{*}{$\mathrm{V}$} & Geological And Petroleum Technicians, And & & & & & & \\
\hline & Nuclear Technicians & 1930 & 1930 & & 2 & 3 & 2 \\
\hline \multirow[t]{2}{*}{$\mathrm{V}$} & Geological And Petroleum Technicians, And & & & & & & \\
\hline & Nuclear Technicians & 1930 & 1940 & $\mathrm{x}$ & 1 & 0 & 0 \\
\hline \multirow[t]{2}{*}{ V } & Health Practitioner Support Technologists & & & & & & \\
\hline & And Technicians & 3420 & 3420 & & 29 & 45 & 44 \\
\hline \multirow[t]{2}{*}{$\mathrm{V}$} & Miscellaneous Health Technologists And & & & & & & \\
\hline & Technicians & 3535 & 3535 & & 5 & 7 & 7 \\
\hline V & Miscellaneous Law Enforcement Workers & 3840 & 3840 & & 1 & 0 & 1 \\
\hline $\mathrm{V}$ & Miscellaneous Law Enforcement Workers & 3840 & 3830 & $\mathrm{x}$ & 1 & 0 & 0 \\
\hline \multirow[t]{2}{*}{$\mathrm{V}$} & $\begin{array}{l}\text { Miscellaneous Life, Physical, And Social } \\
\text { Science Technicians, Including Social Science }\end{array}$ & & & & & & \\
\hline & Research Assistants & 1965 & 1965 & & 23 & 25 & 8 \\
\hline \multirow[t]{2}{*}{$\mathrm{V}$} & $\begin{array}{l}\text { Miscellaneous Life, Physical, And Social } \\
\text { Science Technicians, Including Social Science }\end{array}$ & & & & & & \\
\hline & Research Assistants & 1965 & 1950 & $\mathrm{x}$ & 0 & 1 & 1 \\
\hline \multirow[t]{2}{*}{$\mathrm{V}$} & Miscellaneous Media And Communication & & & & & & \\
\hline & Workers & 2860 & 2860 & & 6 & 7 & 8 \\
\hline V & Musicians, Singers, And Related Workers & 2750 & 2750 & & 30 & 16 & 13 \\
\hline V & Opticians, Dispensing & 3520 & 3520 & & 3 & 4 & 7 \\
\hline V & Photographers & 2910 & 2910 & & 7 & 18 & 12 \\
\hline V & Police Officers & 3850 & 3850 & & 53 & 50 & 42 \\
\hline $\mathrm{V}$ & Private Detectives And Investigators & 3910 & 3910 & & 7 & 9 & 5 \\
\hline \multirow[t]{2}{*}{$\mathrm{V}$} & Probation Officers And Correctional & & & & & & \\
\hline & Treatment Specialists & 2015 & 2015 & & 3 & 7 & 4 \\
\hline \multirow[t]{2}{*}{ V } & Property, Real Estate, And Community & & & & & & \\
\hline & Association Managers & 410 & 410 & & 42 & 36 & 34 \\
\hline \multirow[t]{2}{*}{$\mathrm{V}$} & Radio And Telecommunications Equipment & & & & & & \\
\hline & Installers And Repairers & 7020 & 7020 & & 8 & 20 & 11 \\
\hline V & Railroad Conductors And Yardmasters & 9240 & 9240 & & 8 & 2 & 5 \\
\hline V & Ship And Boat Captains And Operators & 9310 & 9310 & & 2 & 1 & 1 \\
\hline \multirow[t]{2}{*}{$\mathrm{V}$} & Supervisors Of Transportation And Material & & & & & & \\
\hline & Moving Workers & 9000 & 9000 & & 20 & 25 & 15 \\
\hline $\mathrm{V}$ & Surveying And Mapping Technicians & 1560 & 1560 & & 3 & 6 & 6 \\
\hline
\end{tabular}




\begin{tabular}{|c|c|c|c|c|c|c|c|}
\hline Class & Occupational Title (ACS 2012) & $\begin{array}{l}\text { ACS } \\
2012\end{array}$ & $\begin{array}{r}\text { GSS } \\
\text { occ10 }\end{array}$ & $\begin{array}{l}\text { Recoded } \\
\text { to ACS }\end{array}$ & $\begin{array}{r}\text { GSS } \\
1994- \\
2000 \\
\end{array}$ & $\begin{array}{r}\text { GSS } \\
2002- \\
2008 \\
\end{array}$ & $\begin{array}{r}\text { GSS } \\
2010- \\
2016 \\
\end{array}$ \\
\hline \multirow[t]{2}{*}{$\mathrm{V}$} & Telecommunications Line Installers And & & & & & & \\
\hline & Repairers & 7420 & 7420 & & 13 & 15 & 8 \\
\hline \multirow[t]{2}{*}{$\mathrm{V}$} & Television, Video, And Motion Picture Camera & & & & & & \\
\hline & Operators And Editors & 2920 & 2920 & & 5 & 3 & 2 \\
\hline $\mathrm{V}$ & Transportation Inspectors & 9410 & 9410 & & 7 & 4 & 4 \\
\hline \multirow[t]{2}{*}{ V } & Transportation, Storage, And Distribution & & & & & & \\
\hline & Managers & 160 & 160 & & 25 & 16 & 16 \\
\hline VI & Aircraft Mechanics And Service Technicians & 7140 & 7140 & & 17 & 10 & 13 \\
\hline \multirow[t]{2}{*}{ VI } & Automotive Service Technicians And & & & & & & \\
\hline & Mechanics & 7200 & 7200 & & 72 & 73 & 49 \\
\hline VI & Boilermakers & 6210 & 6210 & & 2 & 1 & 2 \\
\hline \multirow[t]{2}{*}{ VI } & Brickmasons, Blockmasons, Stonemasons, And & & & & & & \\
\hline & Reinforcing Iron And Rebar Workers & 6220 & 6220 & & 14 & 13 & 15 \\
\hline \multirow[t]{2}{*}{ VI } & Brickmasons, Blockmasons, Stonemasons, And & & & & & & \\
\hline & Reinforcing Iron And Rebar Workers & 6220 & 6500 & $\mathrm{x}$ & 2 & 1 & 0 \\
\hline \multirow[t]{2}{*}{ VI } & Bus And Truck Mechanics And Diesel Engine & & & & & & \\
\hline & Specialists & 7210 & 7210 & & 25 & 29 & 12 \\
\hline VI & Cabinetmakers And Bench Carpenters & 8500 & 8500 & & 5 & 14 & 4 \\
\hline VI & Carpenters & 6230 & 6230 & & 98 & 124 & 65 \\
\hline \multirow[t]{2}{*}{ VI } & Carpet, Floor, And Tile Installers And & & & & & & \\
\hline & Finishers & 6240 & 6240 & & 20 & 12 & 12 \\
\hline \multirow[t]{2}{*}{ VI } & Computer, Automated Teller, And Office & & & & & & \\
\hline & Machine Repairers & 7010 & 7010 & & 15 & 23 & 11 \\
\hline \multirow[t]{2}{*}{ VI } & Construction Equipment Operators, Except & & & & & & \\
\hline & $\begin{array}{l}\text { Paving, Surfacing, And Tamping Equipment } \\
\text { Operators }\end{array}$ & 6320 & 6320 & & 34 & 34 & 26 \\
\hline VI & Control And Valve Installers And Repairers & 7300 & 7300 & & 0 & 3 & 0 \\
\hline VI & Crane And Tower Operators & 9510 & 9510 & & 7 & 7 & 7 \\
\hline \multirow[t]{2}{*}{ VI } & Drywall Installers, Ceiling Tile Installers, And & & & & & & \\
\hline & Tapers & 6330 & 6330 & & 8 & 12 & 6 \\
\hline VI & Electrical Power-Line Installers And Repairers & 7410 & 7410 & & 11 & 4 & 10 \\
\hline VI & Electricians & 6355 & 6355 & & 62 & 46 & 39 \\
\hline VI & Etchers And Engravers & 8910 & 8910 & & 1 & 0 & 0 \\
\hline \multirow[t]{2}{*}{ VI } & Explosives Workers, Ordnance Handling & & & & & & \\
\hline & Experts, And Blasters & 6830 & 6830 & & 3 & 1 & 3 \\
\hline VI & Forest And Conservation Workers & 6120 & 6120 & & 0 & 2 & 1 \\
\hline VI & Hazardous Materials Removal Workers & 6720 & 6720 & & 5 & 1 & 3 \\
\hline \multirow[t]{2}{*}{ VI } & Heating, Air Conditioning, And Refrigeration & & & & & & \\
\hline & Mechanics And Installers & 7315 & 7315 & & 23 & 30 & 21 \\
\hline \multirow[t]{2}{*}{ VI } & Heavy Vehicle And Mobile Equipment Service & & & & & & \\
\hline & Technicians And Mechanics & 7220 & 7220 & & 11 & 20 & 6 \\
\hline VI & Home Appliance Repairers & 7320 & 7320 & & 7 & 2 & 6 \\
\hline \multirow[t]{2}{*}{ VI } & Industrial And Refractory Machinery & & & & & & \\
\hline & Mechanics & 7330 & 7330 & & 26 & 29 & 9 \\
\hline VI & Insulation Workers & 6400 & 6400 & & 1 & 4 & 4 \\
\hline \multirow[t]{2}{*}{ VI } & Jewelers And Precious Stone And Metal & & & & & & \\
\hline & Workers & 8750 & 8750 & & 2 & 3 & 4 \\
\hline
\end{tabular}




\begin{tabular}{|c|c|c|c|c|c|c|c|}
\hline Class & Occupational Title (ACS 2012) & $\begin{array}{l}\text { ACS } \\
2012\end{array}$ & $\begin{array}{r}\text { GSS } \\
\text { occ10 }\end{array}$ & $\begin{array}{l}\text { Recoded } \\
\text { to ACS }\end{array}$ & $\begin{array}{r}\text { GSS } \\
1994- \\
2000 \\
\end{array}$ & $\begin{array}{r}\text { GSS } \\
2002- \\
2008 \\
\end{array}$ & $\begin{array}{r}\text { GSS } \\
2010- \\
2016 \\
\end{array}$ \\
\hline VI & Locksmiths And Safe Repairers & 7540 & 7540 & & 2 & 4 & 2 \\
\hline VI & Locomotive Engineers And Operators & 9200 & 9200 & & 5 & 4 & 3 \\
\hline VI & Machinists & 8030 & 8030 & & 28 & 33 & 24 \\
\hline VI & Maintenance And Repair Workers, General & 7340 & 7340 & & 15 & 14 & 17 \\
\hline VI & Maintenance Workers, Machinery & 7350 & 7350 & & 2 & 4 & 2 \\
\hline \multirow[t]{2}{*}{ VI } & Medical, Dental, And Ophthalmic Laboratory & & & & & & \\
\hline & Technicians & 8760 & 8760 & & 8 & 7 & 5 \\
\hline VI & Millwrights & 7360 & 7360 & & 5 & 9 & 10 \\
\hline \multirow[t]{2}{*}{ VI } & $\begin{array}{l}\text { Miscellaneous Installation, Maintenance, And } \\
\text { Repair Workers, Including Wind Turbine }\end{array}$ & & & & & & \\
\hline & Service Technicians & 7630 & 7630 & & 12 & 19 & 10 \\
\hline \multirow[t]{2}{*}{ VI } & $\begin{array}{l}\text { Miscellaneous Installation, Maintenance, And } \\
\text { Repair Workers, Including Wind Turbine }\end{array}$ & & & & & & \\
\hline & Service Technicians & 7630 & 7550 & $\mathrm{x}$ & 3 & 0 & 0 \\
\hline VI & Miscellaneous Plant And System Operators & 8630 & 8630 & & 3 & 6 & 5 \\
\hline \multirow[t]{2}{*}{ VI } & Miscellaneous Woodworkers, Including & & & & & & \\
\hline & Model Makers And Patternmakers & 8550 & 8550 & & 4 & 3 & 0 \\
\hline \multirow[t]{2}{*}{ VI } & Miscellaneous Woodworkers, Including & & & & & & \\
\hline & Model Makers And Patternmakers & 8550 & 8520 & $\mathrm{x}$ & 1 & 0 & 0 \\
\hline \multirow[t]{2}{*}{ VI } & Model Makers, Patternmakers, And Molding & & & & & & \\
\hline & Machine Setters, Metal And Plastic & 8100 & 8100 & & 16 & 11 & 6 \\
\hline \multirow[t]{2}{*}{ VI } & Model Makers, Patternmakers, And Molding & & & & & & \\
\hline & Machine Setters, Metal And Plastic & 8100 & 8060 & $\mathrm{x}$ & 1 & 1 & 0 \\
\hline VI & $\begin{array}{l}\text { Molders, Shapers, And Casters, Except Metal } \\
\text { And Plastic }\end{array}$ & 8920 & 8920 & & 2 & 7 & 0 \\
\hline VI & Painters And Paperhangers & 6420 & 6420 & & 30 & 32 & 31 \\
\hline VI & Painters And Paperhangers & 6420 & 6430 & $\mathrm{x}$ & 4 & 1 & 0 \\
\hline \multirow[t]{2}{*}{ VI } & Pipelayers, Plumbers, Pipefitters, And & & & & & & \\
\hline & Steamfitters & 6440 & 6440 & & 65 & 46 & 34 \\
\hline VI & $\begin{array}{l}\text { Power Plant Operators, Distributors, And } \\
\text { Dispatchers }\end{array}$ & 8600 & 8600 & & 3 & 3 & 0 \\
\hline \multirow[t]{2}{*}{ VI } & Precision Instrument And Equipment & & & & & & \\
\hline & Repairers & 7430 & 7430 & & 8 & 10 & 1 \\
\hline VI & Prepress Technicians And Workers & 8250 & 8250 & & 10 & 8 & 6 \\
\hline VI & Printing Press Operators & 8255 & 8255 & & 33 & 30 & 11 \\
\hline \multirow[t]{2}{*}{ VI } & Sailors And Marine Oilers, And Ship & & & & & & \\
\hline & Engineers & 9300 & 9300 & & 2 & 2 & 2 \\
\hline VI & Security And Fire Alarm Systems Installers & 7130 & 7130 & & 3 & 1 & 3 \\
\hline VI & Sheet Metal Workers & 6520 & 6520 & & 8 & 14 & 7 \\
\hline VI & Small Engine Mechanics & 7240 & 7240 & & 2 & 5 & 6 \\
\hline VI & Stationary Engineers And Boiler Operators & 8610 & 8610 & & 14 & 8 & 12 \\
\hline VI & Structural Iron And Steel Workers & 6530 & 6530 & & 8 & 5 & 9 \\
\hline VI & Structural Metal Fabricators And Fitters & 7740 & 7740 & & 7 & 5 & 5 \\
\hline \multirow[t]{2}{*}{ VI } & Subway, Streetcar, And Other Rail & & & & & & \\
\hline & Transportation Workers & 9260 & 9260 & & 3 & 1 & 1 \\
\hline \multirow[t]{2}{*}{ VI } & Subway, Streetcar, And Other Rail & & & & & & \\
\hline & Transportation Workers & 9260 & 9230 & $x$ & 1 & 2 & 0 \\
\hline
\end{tabular}




\begin{tabular}{|c|c|c|c|c|c|c|c|}
\hline Class & Occupational Title (ACS 2012) & $\begin{array}{l}\text { ACS } \\
2012\end{array}$ & $\begin{array}{r}\text { GSS } \\
\text { occ10 }\end{array}$ & $\begin{array}{l}\text { Recoded } \\
\text { to ACS }\end{array}$ & $\begin{array}{r}\text { GSS } \\
1994- \\
2000\end{array}$ & $\begin{array}{r}\text { GSS } \\
2002- \\
2008\end{array}$ & $\begin{array}{r}\text { GSS } \\
2010- \\
2016\end{array}$ \\
\hline VI & Tailors, Dressmakers, And Sewers & 8350 & 8350 & & 16 & 15 & 12 \\
\hline VI & Tool And Die Makers & 8130 & 8130 & & 10 & 10 & 9 \\
\hline VI & $\begin{array}{l}\text { Water And Wastewater Treatment Plant And } \\
\text { System Operators }\end{array}$ & 8620 & 8620 & & 9 & 3 & 6 \\
\hline VIIa & $\begin{array}{l}\text { Adhesive Bonding Machine Operators And } \\
\text { Tenders }\end{array}$ & 8850 & 8850 & & 3 & 2 & 0 \\
\hline VIIa & $\begin{array}{l}\text { Aircraft Structure, Surfaces, Rigging, And } \\
\text { Systems Assemblers }\end{array}$ & 7710 & 7710 & & 4 & 5 & 1 \\
\hline VIIa & Automotive Body And Related Repairers & 7150 & 7150 & & 20 & 15 & 13 \\
\hline VIIa & Automotive Glass Installers And Repairers & 7160 & 7160 & & 3 & 2 & 2 \\
\hline VIIa & Bakers & 7800 & 7800 & & 21 & 14 & 13 \\
\hline VIIa & $\begin{array}{l}\text { Butchers And Other Meat, Poultry, And Fish } \\
\text { Processing Workers } \\
\text { Cement Masons, Concrete Finishers, And }\end{array}$ & 7810 & 7810 & & 18 & 23 & 12 \\
\hline VIIa & $\begin{array}{l}\text { Terrazzo Workers } \\
\text { Chemical Processing Machine Setters, } \\
\text { Operators, And Tenders }\end{array}$ & 6250 & 6250 & & 10 & 5 & 5 \\
\hline VIIa & Cleaners Of Vehicles And Equipment & 9610 & 9610 & & 26 & 16 & 9 \\
\hline VIIa & Construction Laborers & 6260 & 6260 & & 69 & 117 & 104 \\
\hline VIIa & $\begin{array}{l}\text { Conveyor Operators And Tenders, And Hoist } \\
\text { And Winch Operators }\end{array}$ & 9560 & 9500 & $\mathrm{x}$ & 0 & 1 & 0 \\
\hline VIIa & Cooks & 4020 & 4020 & & 121 & 153 & 116 \\
\hline VIIa & Crossing Guards & 3940 & 3940 & & 6 & 6 & 3 \\
\hline VIIa & $\begin{array}{l}\text { Crushing, Grinding, Polishing, Mixing, And } \\
\text { Blending Workers }\end{array}$ & 8650 & 8650 & & 7 & 5 & 6 \\
\hline VIIa & Cutting Workers & 8710 & 8710 & & 11 & 8 & 6 \\
\hline VIIa & $\begin{array}{l}\text { Derrick, Rotary Drill, And Service Unit } \\
\text { Operators, And Roustabouts, Oil, Gas, And }\end{array}$ & & & & & & \\
\hline & Mining & 6800 & 6800 & & 0 & 5 & 0 \\
\hline VIIa & Dishwashers & 4140 & 4140 & & 12 & 26 & 12 \\
\hline VIIa & $\begin{array}{l}\text { Dredge, Excavating, And Loading Machine } \\
\text { Operators }\end{array}$ & 9520 & 9520 & & 1 & 9 & 4 \\
\hline VIIa & Driver/Sales Workers And Truck Drivers & 9130 & 9130 & & 212 & 243 & 179 \\
\hline VIIa & Earth Drillers, Except Oil And Gas & 6820 & 6820 & & 3 & 2 & 1 \\
\hline VIIa & $\begin{array}{l}\text { Electrical, Electronics, And Electromechanical } \\
\text { Assemblers }\end{array}$ & 7720 & 7720 & & 49 & 22 & 15 \\
\hline VIIa & Engine And Other Machine Assemblers & 7730 & 7730 & & 6 & 1 & 6 \\
\hline VIIa & $\begin{array}{l}\text { Extruding And Drawing Machine Setters, } \\
\text { Operators, And Tenders, Metal And Plastic } \\
\text { Extruding, Forming, Pressing, And } \\
\text { Compacting Machine Setters, Operators, And }\end{array}$ & 7920 & 7920 & & 1 & 1 & 3 \\
\hline & Tenders & 8720 & 8720 & & 8 & 7 & 3 \\
\hline VIIa & Fence Erectors & 6710 & 6710 & & 1 & 2 & 0 \\
\hline VIIa & $\begin{array}{l}\text { Food And Tobacco Roasting, Baking, And } \\
\text { Drying Machine Operators And Tenders }\end{array}$ & 7830 & 7830 & & 1 & 2 & 4 \\
\hline VIIa & Food Batchmakers & 7840 & 7840 & & 4 & 9 & 6 \\
\hline
\end{tabular}




\begin{tabular}{|c|c|c|c|c|c|c|c|}
\hline Class & Occupational Title (ACS 2012) & $\begin{array}{l}\text { ACS } \\
2012\end{array}$ & $\begin{array}{r}\text { GSS } \\
\text { occ10 }\end{array}$ & $\begin{array}{l}\text { Recoded } \\
\text { to ACS }\end{array}$ & $\begin{array}{r}\text { GSS } \\
1994- \\
2000\end{array}$ & $\begin{array}{r}\text { GSS } \\
2002- \\
2008\end{array}$ & $\begin{array}{r}\text { GSS } \\
2010- \\
2016\end{array}$ \\
\hline \multirow[t]{2}{*}{ VIIa } & Food Cooking Machine Operators And & & & & & & \\
\hline & Tenders & 7850 & 7850 & & 4 & 0 & 1 \\
\hline VIIa & Food Preparation Workers & 4030 & 4030 & & 34 & 31 & 44 \\
\hline VIIa & Food Processing Workers, All Other & 7855 & 7855 & & 5 & 11 & 9 \\
\hline \multirow[t]{2}{*}{ VIIa } & Forging Machine Setters, Operators, And & & & & & & \\
\hline & Tenders, Metal And Plastic & 7930 & 7930 & & 2 & 0 & 2 \\
\hline \multirow[t]{2}{*}{ VIIa } & Furnace, Kiln, Oven, Drier, And Kettle & & & & & & \\
\hline & Operators And Tenders & 8730 & 8730 & & 2 & 1 & 0 \\
\hline VIIa & Furniture Finishers & 8510 & 8510 & & 3 & 6 & 2 \\
\hline VIIa & Glaziers & 6360 & 6360 & & 0 & 5 & 5 \\
\hline VIIa & Grounds Maintenance Workers & 4250 & 4250 & & 56 & 56 & 72 \\
\hline VIIa & Helpers, Construction Trades & 6600 & 6600 & & 7 & 5 & 4 \\
\hline \multirow[t]{2}{*}{ VIIa } & Helpers-Installation, Maintenance, And Repair & & & & & & \\
\hline & Workers & 7610 & 7610 & & 3 & 1 & 5 \\
\hline VIIa & Helpers-Production Workers & 8950 & 8950 & & 4 & 1 & 6 \\
\hline VIIa & Highway Maintenance Workers & 6730 & 6730 & & 5 & 6 & 6 \\
\hline VIIa & Industrial Truck And Tractor Operators & 9600 & 9600 & & 36 & 43 & 29 \\
\hline \multirow[t]{2}{*}{ VIIa } & Inspectors, Testers, Sorters, Samplers, And & & & & & & \\
\hline & Weighers & 8740 & 8740 & & 88 & 79 & 41 \\
\hline VIIa & Janitors And Building Cleaners & 4220 & 4220 & & 165 & 182 & 171 \\
\hline \multirow[t]{2}{*}{ VIIa } & Laborers And Freight, Stock, And Material & & & & & & \\
\hline & Movers, Hand & 9620 & 9620 & & 107 & 107 & 99 \\
\hline VIIa & Laundry And Dry-Cleaning Workers & 8300 & 8300 & & 31 & 15 & 14 \\
\hline VIIa & Logging Workers & 6130 & 6130 & & 7 & 20 & 9 \\
\hline VIIa & Machine Feeders And Offbearers & 9630 & 9630 & & 8 & 14 & 1 \\
\hline \multirow[t]{2}{*}{ VIIa } & Machine Tool Cutting Setters, Operators, And & & & & & & \\
\hline & Tenders, Metal And Plastic & 7950 & 7950 & & 18 & 18 & 8 \\
\hline \multirow[t]{2}{*}{ VIIa } & Machine Tool Cutting Setters, Operators, And & & & & & & \\
\hline & Tenders, Metal And Plastic & 7950 & 8000 & $x$ & 3 & 8 & 3 \\
\hline \multirow[t]{2}{*}{ VIIa } & Machine Tool Cutting Setters, Operators, And & & & & & & \\
\hline & Tenders, Metal And Plastic & 7950 & 8010 & $x$ & 8 & 6 & 0 \\
\hline \multirow[t]{2}{*}{ VIIa } & Machine Tool Cutting Setters, Operators, And & & & & & & \\
\hline & Tenders, Metal And Plastic & 7950 & 7960 & $x$ & 3 & 3 & 1 \\
\hline VIIa & Maids And Housekeeping Cleaners & 4230 & 4230 & & 179 & 160 & 132 \\
\hline VIIa & Mail Clerks And Mail Machine Operators, & & & & & & \\
\hline \multirow{3}{*}{ VIIa } & Except Postal Service & 5850 & 5850 & & 15 & 13 & 2 \\
\hline & Metal Furnace Operators, Tenders, Pourers, & & & & & & \\
\hline & And Casters & 8040 & 8040 & & 2 & 4 & 0 \\
\hline VIIa & Mining Machine Operators & 6840 & 6840 & & 1 & 4 & 5 \\
\hline VIIa & Miscellaneous Assemblers And Fabricators & 7750 & 7750 & & 100 & 77 & 54 \\
\hline VIIa & $\begin{array}{l}\text { Miscellaneous Construction Workers, } \\
\text { Including Solar Photovoltaic Installers, Septic }\end{array}$ & & & & & & \\
\hline \multirow{3}{*}{ VIIa } & Tank Servicers And Sewer Pipe Cleaners & 6765 & 6765 & & 4 & 4 & 2 \\
\hline & $\begin{array}{l}\text { Miscellaneous Construction Workers, } \\
\text { Including Solar Photovoltaic Installers, Septic }\end{array}$ & & & & & & \\
\hline & Tank Servicers And Sewer Pipe Cleaners & 6765 & 6750 & $x$ & 0 & 0 & 1 \\
\hline
\end{tabular}




\begin{tabular}{|c|c|c|c|c|c|c|c|}
\hline Class & Occupational Title (ACS 2012) & $\begin{array}{l}\text { ACS } \\
2012\end{array}$ & $\begin{array}{r}\text { GSS } \\
\text { occ10 }\end{array}$ & $\begin{array}{l}\text { Recoded } \\
\text { to ACS }\end{array}$ & $\begin{array}{r}\text { GSS } \\
1994- \\
2000\end{array}$ & $\begin{array}{r}\text { GSS } \\
2002- \\
2008\end{array}$ & $\begin{array}{r}\text { GSS } \\
2010- \\
2016\end{array}$ \\
\hline \multirow[t]{2}{*}{ VIIa } & Miscellaneous Extraction Workers, Including & & & & & & \\
\hline & Roof Bolters And Helpers & 6940 & 6940 & & 4 & 2 & 1 \\
\hline \multirow[t]{2}{*}{ VIIa } & Miscellaneous Extraction Workers, Including & & & & & & \\
\hline & Roof Bolters And Helpers & 6940 & 6930 & $\mathrm{x}$ & 2 & 1 & 0 \\
\hline \multirow[t]{2}{*}{ VIIa } & $\begin{array}{l}\text { Miscellaneous Material Moving Workers, } \\
\text { Including Mine Shuttle Car Operators, And }\end{array}$ & & & & & & \\
\hline & Tank Car, Truck, And Ship Loaders & 9750 & 9750 & & 8 & 8 & 7 \\
\hline \multirow[t]{2}{*}{ VIIa } & $\begin{array}{l}\text { Miscellaneous Material Moving Workers, } \\
\text { Including Mine Shuttle Car Operators, And }\end{array}$ & & & & & & \\
\hline & Tank Car, Truck, And Ship Loaders & 9750 & 9730 & $x$ & 0 & 2 & 0 \\
\hline \multirow[t]{2}{*}{ VIIa } & $\begin{array}{l}\text { Miscellaneous Metal Workers And Plastic } \\
\text { Workers, Including Multiple Machine Tool }\end{array}$ & & & & & & \\
\hline & Setters & 8220 & 8220 & & 33 & 24 & 22 \\
\hline \multirow[t]{2}{*}{ VIIa } & $\begin{array}{l}\text { Miscellaneous Metal Workers And Plastic } \\
\text { Workers, Including Multiple Machine Tool }\end{array}$ & & & & & & \\
\hline & Setters & 8220 & 8210 & $x$ & 1 & 2 & 0 \\
\hline \multirow[t]{2}{*}{ VIIa } & $\begin{array}{l}\text { Miscellaneous Metal Workers And Plastic } \\
\text { Workers, Including Multiple Machine Tool }\end{array}$ & & & & & & \\
\hline & Setters & 8220 & 8150 & $x$ & 3 & 0 & 1 \\
\hline \multirow[t]{2}{*}{ VIIa } & $\begin{array}{l}\text { Miscellaneous Metal Workers And Plastic } \\
\text { Workers, Including Multiple Machine Tool }\end{array}$ & & & & & & \\
\hline & Setters & 8220 & 8200 & $x$ & 3 & 1 & 0 \\
\hline \multirow[t]{2}{*}{ VIIa } & $\begin{array}{l}\text { Miscellaneous Metal Workers And Plastic } \\
\text { Workers, Including Multiple Machine Tool }\end{array}$ & & & & & & \\
\hline & Setters & 8220 & 8020 & $x$ & 1 & 1 & 0 \\
\hline \multirow[t]{2}{*}{ VIIa } & $\begin{array}{l}\text { Miscellaneous Metal Workers And Plastic } \\
\text { Workers, Including Multiple Machine Tool }\end{array}$ & & & & & & \\
\hline & Setters & 8220 & 8120 & $x$ & 0 & 2 & 0 \\
\hline VIIa & $\begin{array}{l}\text { Miscellaneous Production Workers, Including } \\
\text { Semiconductor Processors }\end{array}$ & 8965 & 8965 & & 124 & 111 & 97 \\
\hline VIIa & $\begin{array}{l}\text { Miscellaneous Production Workers, Including } \\
\text { Semiconductor Processors }\end{array}$ & 8965 & 8840 & $x$ & 1 & 0 & 0 \\
\hline VIIa & $\begin{array}{l}\text { Miscellaneous Production Workers, Including } \\
\text { Semiconductor Processors }\end{array}$ & 8965 & 8860 & $x$ & 0 & 1 & 1 \\
\hline \multirow[t]{2}{*}{ VIIa } & Miscellaneous Production Workers, Including & & & & & & \\
\hline & Semiconductor Processors & 8965 & 8900 & $\mathrm{x}$ & 2 & 0 & 0 \\
\hline \multirow[t]{2}{*}{ VIIa } & Miscellaneous Textile, Apparel, And & & & & & & \\
\hline & Furnishings Workers, Except Upholsterers & 8460 & 8460 & & 9 & 7 & 2 \\
\hline \multirow[t]{2}{*}{ VIIa } & Miscellaneous Textile, Apparel, And & & & & & & \\
\hline & Furnishings Workers, Except Upholsterers & 8460 & 8440 & $\mathrm{x}$ & 2 & 0 & 0 \\
\hline \multirow[t]{2}{*}{ VIIa } & Miscellaneous Vehicle And Mobile Equipment & & & & & & \\
\hline & Mechanics, Installers, And Repairers & 7260 & 7260 & & 1 & 6 & 7 \\
\hline VIIa & Motor Vehicle Operators, All Other & 9150 & 9150 & & 3 & 3 & 0 \\
\hline \multirow[t]{2}{*}{ VIIa } & Packaging And Filling Machine Operators & & & & & & \\
\hline & And Tenders & 8800 & 8800 & & 27 & 17 & 19 \\
\hline VIIa & Packers And Packagers, Hand & 9640 & 9640 & & 35 & 35 & 28 \\
\hline VIIa & Painting Workers & 8810 & 8810 & & 11 & 12 & 10 \\
\hline VIIa & $\begin{array}{l}\text { Paper Goods Machine Setters, Operators, And } \\
\text { Tenders }\end{array}$ & 8930 & 8930 & & 4 & 7 & 2 \\
\hline
\end{tabular}




\begin{tabular}{|c|c|c|c|c|c|c|c|}
\hline Class & Occupational Title (ACS 2012) & $\begin{array}{l}\text { ACS } \\
2012 \\
\end{array}$ & $\begin{array}{r}\text { GSS } \\
\text { occ10 }\end{array}$ & $\begin{array}{l}\text { Recoded } \\
\text { to ACS }\end{array}$ & $\begin{array}{r}\text { GSS } \\
1994- \\
2000\end{array}$ & $\begin{array}{r}\text { GSS } \\
2002- \\
2008\end{array}$ & $\begin{array}{r}\text { GSS } \\
2010- \\
2016\end{array}$ \\
\hline \multirow[t]{2}{*}{ VIIa } & Paving, Surfacing, And Tamping Equipment & & & & & & \\
\hline & Operators & 6300 & 6300 & & 0 & 1 & 1 \\
\hline \multirow[t]{2}{*}{ VIIa } & Photographic Process Workers And Processing & & & & & & \\
\hline & Machine Operators & 8830 & 8830 & & 10 & 9 & 3 \\
\hline VIIa & Plasterers And Stucco Masons & 6460 & 6460 & & 3 & 3 & 0 \\
\hline VIIa & Postal Service Mail Carriers & 5550 & 5550 & & 30 & 21 & 23 \\
\hline \multirow[t]{2}{*}{ VIIa } & Postal Service Mail Sorters, Processors, And & & & & & & \\
\hline & Processing Machine Operators & 5560 & 5560 & & 16 & 13 & 3 \\
\hline \multirow[t]{2}{*}{ VIIa } & Pressers, Textile, Garment, And Related & & & & & & \\
\hline & Materials & 8310 & 8310 & & 15 & 9 & 8 \\
\hline VIIa & Print Binding And Finishing Workers & 8256 & 8256 & & 9 & 4 & 3 \\
\hline VIIa & Pumping Station Operators & 9650 & 9650 & & 2 & 3 & 4 \\
\hline \multirow[t]{2}{*}{ VIIa } & Rail-Track Laying And Maintenance & & & & & & \\
\hline & Equipment Operators & 6740 & 6740 & & 1 & 3 & 0 \\
\hline VIIa & Refuse And Recyclable Material Collectors & 9720 & 9720 & & 3 & 10 & 11 \\
\hline VIIa & Riggers & 7560 & 7560 & & 1 & 0 & 0 \\
\hline \multirow[t]{2}{*}{ VIIa } & Rolling Machine Setters, Operators, And & & & & & & \\
\hline & Tenders, Metal And Plastic & 7940 & 7940 & & 3 & 0 & 1 \\
\hline VIIa & Roofers & 6515 & 6515 & & 11 & 17 & 14 \\
\hline \multirow[t]{2}{*}{ VIIa } & Sawing Machine Setters, Operators, And & & & & & & \\
\hline & Tenders, Wood & 8530 & 8530 & & 11 & 12 & 3 \\
\hline VIIa & Sewing Machine Operators & 8320 & 8320 & & 67 & 61 & 28 \\
\hline VIIa & Shipping, Receiving, And Traffic Clerks & 5610 & 5610 & & 50 & 39 & 25 \\
\hline VIIa & Shoe And Leather Workers & 8330 & 8330 & & 3 & 4 & 0 \\
\hline VIIa & Shoe And Leather Workers & 8330 & 8340 & $x$ & 10 & 0 & 3 \\
\hline VIIa & Stock Clerks And Order Fillers & 5620 & 5620 & & 103 & 84 & 63 \\
\hline VIIa & Taxi Drivers And Chauffeurs & 9140 & 9140 & & 15 & 21 & 20 \\
\hline \multirow[t]{2}{*}{ VIIa } & Textile Bleaching And Dyeing, And Cutting & & & & & & \\
\hline & Machine Setters, Operators, And Tenders & 8400 & 8400 & & 3 & 1 & 0 \\
\hline \multirow[t]{2}{*}{ VIIa } & Textile Bleaching And Dyeing, And Cutting & & & & & & \\
\hline & Machine Setters, Operators, And Tenders & 8400 & 8360 & $x$ & 0 & 0 & 2 \\
\hline \multirow[t]{2}{*}{ VIIa } & Textile Knitting And Weaving Machine & & & & & & \\
\hline & Setters, Operators, And Tenders & 8410 & 8410 & & 8 & 4 & 1 \\
\hline \multirow[t]{2}{*}{ VIIa } & Textile Winding, Twisting, And Drawing Out & & & & & & \\
\hline & Machine Setters, Operators, And Tenders & 8420 & 8420 & & 13 & 12 & 2 \\
\hline VIIa & Tire Builders & 8940 & 8940 & & 2 & 2 & 1 \\
\hline VIIa & Upholsterers & 8450 & 8450 & & 11 & 9 & 0 \\
\hline VIIa & Welding, Soldering, And Brazing Workers & 8140 & 8140 & & 63 & 53 & 41 \\
\hline \multirow[t]{2}{*}{ VIIa } & Woodworking Machine Setters, Operators, & & & & & & \\
\hline & And Tenders, Except Sawing & 8540 & 8540 & & 2 & 7 & 4 \\
\hline VIIb & Agricultural Inspectors & 6010 & 6010 & & 1 & 2 & 2 \\
\hline VIIb & Animal Trainers & 4340 & 4340 & & 1 & 5 & 1 \\
\hline \multirow[t]{2}{*}{ VIIb } & First-Line Supervisors Of Farming, Fishing, & & & & & & \\
\hline & And Forestry Workers & 6005 & 6005 & & 3 & 6 & 4 \\
\hline VIIlb & Fishing And Hunting Workers & 6100 & 6100 & & 3 & 10 & 4 \\
\hline VIIlb & Graders And Sorters, Agricultural Products & 6040 & 6040 & & 5 & 6 & 4 \\
\hline
\end{tabular}




\begin{tabular}{|c|c|c|c|c|c|c|c|}
\hline Class & Occupational Title (ACS 2012) & $\begin{array}{l}\text { ACS } \\
2012\end{array}$ & $\begin{array}{r}\text { GSS } \\
\text { occ10 }\end{array}$ & $\begin{array}{l}\text { Recoded } \\
\text { to ACS }\end{array}$ & $\begin{array}{r}\text { GSS } \\
1994- \\
2000\end{array}$ & $\begin{array}{r}\text { GSS } \\
2002- \\
2008\end{array}$ & $\begin{array}{r}\text { GSS } \\
2010- \\
2016\end{array}$ \\
\hline VIIlb & $\begin{array}{l}\text { Miscellaneous Agricultural Workers, } \\
\text { Including Animal Breeders }\end{array}$ & 6050 & 6050 & & 49 & 73 & 48 \\
\hline VIIb & $\begin{array}{l}\text { Miscellaneous Agricultural Workers, } \\
\text { Including Animal Breeders }\end{array}$ & 6050 & 6020 & $\mathrm{x}$ & 1 & 0 & 0 \\
\hline Mil & First-Line Enlisted Military Supervisors & 9810 & 9810 & & 16 & 15 & 35 \\
\hline Mil & $\begin{array}{l}\text { Military Enlisted Tactical Operations And } \\
\text { Air/Weapons Specialists And Crew Members } \\
\text { Military Officer Special And Tactical }\end{array}$ & 9820 & 9820 & & 8 & 15 & 7 \\
\hline Mil & $\begin{array}{l}\text { Military Officer Special And Tactical } \\
\text { Operations Leaders }\end{array}$ & 9800 & 9800 & & 7 & 10 & 38 \\
\hline Mil & Military, Rank Not Specified & 9830 & 9830 & & 13 & 23 & 41 \\
\hline
\end{tabular}


Table A2. GSS Occupational Distributions for the 11-Class Version of EGP (i.e., with Combined IVab)

\begin{tabular}{|c|c|c|c|c|c|c|c|}
\hline Class & Occupational Title (ACS 2012) & $\begin{array}{l}\text { ACS } \\
2012\end{array}$ & $\begin{array}{r}\text { GSS } \\
\text { occ10 }\end{array}$ & $\begin{array}{l}\text { Recoded } \\
\text { to ACS }\end{array}$ & $\begin{array}{r}\text { GSS } \\
1994- \\
2000 \\
\end{array}$ & $\begin{array}{r}\text { GSS } \\
2002- \\
2008 \\
\end{array}$ & $\begin{array}{r}\text { GSS } \\
2010- \\
2016 \\
\end{array}$ \\
\hline I & Accountants And Auditors & 800 & 800 & & 112 & 120 & 93 \\
\hline I & Actuaries & 1200 & 1200 & & 2 & 1 & 0 \\
\hline I & Aerospace Engineers & 1320 & 1320 & & 7 & 19 & 7 \\
\hline I & Agricultural And Food Scientists & 1600 & 1600 & & 5 & 2 & 4 \\
\hline I & Architects, Except Naval & 1300 & 1300 & & 20 & 16 & 14 \\
\hline I & Architectural And Engineering Managers & 300 & 300 & & 9 & 11 & 4 \\
\hline I & Astronomers And Physicists & 1700 & 1700 & & 0 & 1 & 0 \\
\hline I & Atmospheric And Space Scientists & 1710 & 1710 & & 2 & 0 & 2 \\
\hline I & Biological Scientists & 1610 & 1610 & & 6 & 11 & 8 \\
\hline I & Budget Analysts & 820 & 820 & & 2 & 7 & 3 \\
\hline I & Chemical Engineers & 1350 & 1350 & & 10 & 7 & 2 \\
\hline I & Chemists And Materials Scientists & 1720 & 1720 & & 9 & 12 & 5 \\
\hline I & Chief Executives And Legislators & 10 & 10 & & 74 & 62 & 64 \\
\hline I & Chief Executives And Legislators & 10 & 30 & $x$ & 1 & 4 & 1 \\
\hline I & Chiropractors & 3000 & 3000 & & 0 & 9 & 3 \\
\hline I & Civil Engineers & 1360 & 1360 & & 25 & 29 & 19 \\
\hline \multirow[t]{2}{*}{$\mathrm{I}$} & Computer And Information Research & & & & & & \\
\hline & Scientists & 1005 & 1005 & & 6 & 2 & 0 \\
\hline \multirow[t]{2}{*}{ I } & Computer And Information Systems & & & & & & \\
\hline & Managers & 110 & 110 & & 18 & 20 & 25 \\
\hline I & Computer Hardware Engineers & 1400 & 1400 & & 4 & 5 & 2 \\
\hline I & Conservation Scientists And Foresters & 1640 & 1640 & & 3 & 6 & 3 \\
\hline I & Dentists & 3010 & 3010 & & 10 & 7 & 6 \\
\hline I & Economists & 1800 & 1800 & & 1 & 1 & 3 \\
\hline I & Education Administrators & 230 & 230 & & 44 & 65 & 52 \\
\hline I & Electrical And Electronics Engineers & 1410 & 1410 & & 35 & 29 & 22 \\
\hline I & Environmental Engineers & 1420 & 1420 & & 7 & 8 & 3 \\
\hline I & Environmental Scientists And Geoscientists & 1740 & 1740 & & 4 & 7 & 11 \\
\hline $\mathrm{I}$ & Financial Analysts & 840 & 840 & & 3 & 8 & 9 \\
\hline \multirow[t]{2}{*}{ I } & Industrial Engineers, Including Health And & & & & & & \\
\hline & Safety & 1430 & 1430 & & 20 & 24 & 18 \\
\hline I & Judicial Law Clerks & 2105 & 2105 & & 0 & 1 & 2 \\
\hline I & $\begin{array}{l}\text { Lawyers, And Judges, Magistrates, And Other } \\
\text { Judicial Workers }\end{array}$ & 2100 & 2100 & & 51 & 62 & 46 \\
\hline \multirow[t]{2}{*}{$\mathrm{I}$} & Lawyers, And Judges, Magistrates, And Other & & & & & & \\
\hline & Judicial Workers & 2100 & 2110 & $x$ & 7 & 5 & 1 \\
\hline $\mathrm{I}$ & Management Analysts & 710 & 710 & & 45 & 56 & 44 \\
\hline I & Marine Engineers And Naval Architects & 1440 & 1440 & & 0 & 1 & 0 \\
\hline I & Materials Engineers & 1450 & 1450 & & 1 & 2 & 2 \\
\hline $\mathrm{I}$ & Mechanical Engineers & 1460 & 1460 & & 25 & 34 & 28 \\
\hline I & Medical And Health Services Managers & 350 & 350 & & 37 & 30 & 45 \\
\hline $\mathrm{I}$ & $\begin{array}{l}\text { Medical Scientists, And Life Scientists, All } \\
\text { Other }\end{array}$ & 1650 & 1650 & & 4 & 6 & 12 \\
\hline
\end{tabular}




\begin{tabular}{|c|c|c|c|c|c|c|c|}
\hline Class & Occupational Title (ACS 2012) & $\begin{array}{l}\text { ACS } \\
2012\end{array}$ & $\begin{array}{r}\text { GSS } \\
\text { occ10 }\end{array}$ & $\begin{array}{l}\text { Recoded } \\
\text { to ACS }\end{array}$ & $\begin{array}{r}\text { GSS } \\
1994- \\
2000\end{array}$ & $\begin{array}{r}\text { GSS } \\
2002- \\
2008\end{array}$ & $\begin{array}{r}\text { GSS } \\
2010- \\
2016\end{array}$ \\
\hline \multirow[t]{2}{*}{ I } & Miscellaneous Engineers, Including Nuclear & & & & & & \\
\hline & Engineers & 1530 & 1530 & & 9 & 13 & 10 \\
\hline \multirow[t]{2}{*}{$\mathrm{I}$} & Miscellaneous Engineers, Including Nuclear & & & & & & \\
\hline & Engineers & 1530 & 1510 & $\mathrm{x}$ & 0 & 0 & 3 \\
\hline \multirow[t]{2}{*}{ I } & $\begin{array}{l}\text { Miscellaneous Mathematical Science } \\
\text { Occupations, Including Mathematicians And }\end{array}$ & & & & & & \\
\hline & Statisticians & 1240 & 1210 & $\mathrm{x}$ & 0 & 2 & 1 \\
\hline \multirow[t]{2}{*}{ I } & $\begin{array}{l}\text { Miscellaneous Mathematical Science } \\
\text { Occupations, Including Mathematicians And }\end{array}$ & & & & & & \\
\hline & Statisticians & 1240 & 1230 & $x$ & 2 & 3 & 0 \\
\hline I & Natural Sciences Managers & 360 & 360 & & 1 & 1 & 3 \\
\hline I & Operations Research Analysts & 1220 & 1220 & & 9 & 13 & 7 \\
\hline I & Optometrists & 3040 & 3040 & & 3 & 1 & 1 \\
\hline I & $\begin{array}{l}\text { Petroleum, Mining And Geological Engineers, } \\
\text { Including Mining Safety Engineers }\end{array}$ & 1520 & 1520 & & 0 & 3 & 0 \\
\hline $\mathrm{I}$ & $\begin{array}{l}\text { Petroleum, Mining And Geological Engineers, } \\
\text { Including Mining Safety Enoineers }\end{array}$ & & 100 & 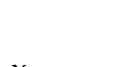 & 1 & 0 & 1 \\
\hline I & Pharmacists & 3050 & 3050 & & 12 & 11 & 12 \\
\hline I & Physical Scientists, All Other & 1760 & 1760 & & 3 & 5 & 9 \\
\hline I & Physicians And Surgeons & 3060 & 3060 & & 38 & 43 & 33 \\
\hline I & Podiatrists & 3120 & 3120 & & 2 & 1 & 0 \\
\hline I & Postsecondary Teachers & 2200 & 2200 & & 98 & 117 & 105 \\
\hline I & Psychologists & 1820 & 1820 & & 15 & 13 & 15 \\
\hline \multirow[t]{2}{*}{ I } & Software Developers, Applications And & & & & & & \\
\hline & Systems Software & 1020 & 1020 & & 44 & 74 & 61 \\
\hline I & Urban And Regional Planners & 1840 & 1840 & & 3 & 7 & 1 \\
\hline I & Veterinarians & 3250 & 3250 & & 5 & 4 & 3 \\
\hline II & Advertising And Promotions Managers & 40 & 40 & & 12 & 4 & 2 \\
\hline \multirow[t]{2}{*}{ II } & Agents And Business Managers Of Artists, & & & & & & \\
\hline & Performers, And Athletes & 500 & 500 & & 7 & 5 & 4 \\
\hline II & Aircraft Pilots And Flight Engineers & 9030 & 9030 & & 17 & 13 & 7 \\
\hline \multirow[t]{2}{*}{ II } & Archivists, Curators, And Museum & & & & & & \\
\hline & Technicians & 2400 & 2400 & & 2 & 8 & 6 \\
\hline II & Business Operations Specialists, All Other & 740 & 740 & & 17 & 30 & 24 \\
\hline II & Clergy & 2040 & 2040 & & 28 & 28 & 20 \\
\hline II & Compensation And Benefits Managers & 135 & 135 & & 2 & 5 & 4 \\
\hline \multirow[t]{2}{*}{ II } & Compensation, Benefits, And Job Analysis & & & & & & \\
\hline & Specialists & 640 & 640 & & 4 & 4 & 7 \\
\hline II & Compliance Officers & 565 & 565 & & 10 & 6 & 16 \\
\hline II & Computer Network Architects & 1106 & 1106 & & 2 & 12 & 5 \\
\hline II & Computer Occupations, All Other & 1107 & 1107 & & 6 & 8 & 13 \\
\hline II & Computer Programmers & 1010 & 1010 & & 53 & 45 & 17 \\
\hline II & Computer Systems Analysts & 1006 & 1006 & & 36 & 35 & 34 \\
\hline II & Counselors & 2000 & 2000 & & 46 & 66 & 39 \\
\hline II & Database Administrators & 1060 & 1060 & & 8 & 10 & 3 \\
\hline II & Dietitians And Nutritionists & 3030 & 3030 & & 9 & 9 & 7 \\
\hline
\end{tabular}




\begin{tabular}{|c|c|c|c|c|c|c|c|}
\hline Class & Occupational Title (ACS 2012) & $\begin{array}{l}\text { ACS } \\
2012\end{array}$ & $\begin{array}{r}\text { GSS } \\
\text { occ10 }\end{array}$ & $\begin{array}{l}\text { Recoded } \\
\text { to ACS }\end{array}$ & $\begin{array}{r}\text { GSS } \\
1994- \\
2000\end{array}$ & $\begin{array}{r}\text { GSS } \\
2002- \\
2008\end{array}$ & $\begin{array}{r}\text { GSS } \\
2010- \\
2016\end{array}$ \\
\hline II & Directors, Religious Activities And Education & 2050 & 2050 & & 1 & 6 & 1 \\
\hline II & Editors & 2830 & 2830 & & 12 & 16 & 16 \\
\hline II & Elementary And Middle School Teachers & 2310 & 2310 & & 205 & 254 & 193 \\
\hline II & Emergency Management Directors & 425 & 425 & & 1 & 1 & 0 \\
\hline II & Financial Examiners & 900 & 900 & & 2 & 1 & 1 \\
\hline II & Financial Managers & 120 & 120 & & 64 & 76 & 66 \\
\hline II & Financial Specialists, All Other & 950 & 950 & & 3 & 3 & 4 \\
\hline II & Fundraisers & 726 & 726 & & 4 & 6 & 2 \\
\hline II & General And Operations Managers & 20 & 20 & & 53 & 56 & 74 \\
\hline II & $\begin{array}{l}\text { Health Diagnosing And Treating Practitioners, } \\
\text { All Other }\end{array}$ & 3260 & 3260 & & 1 & 3 & 2 \\
\hline II & Human Resources Managers & 136 & 136 & & 17 & 16 & 16 \\
\hline II & Industrial Production Managers & 140 & 140 & & 31 & 24 & 20 \\
\hline II & Information Security Analysts & 1007 & 1007 & & 2 & 5 & 3 \\
\hline II & Insurance Underwriters & 860 & 860 & & 4 & 7 & 8 \\
\hline II & Librarians & 2430 & 2430 & & 19 & 19 & 25 \\
\hline II & Logisticians & 700 & 700 & & 1 & 3 & 10 \\
\hline II & Market Research Analysts And Marketing & & & & & & \\
\hline & Specialists & 735 & 735 & & 13 & 12 & 12 \\
\hline II & Marketing And Sales Managers & 50 & 50 & & 76 & 78 & 51 \\
\hline II & $\begin{array}{l}\text { Miscellaneous Community And Social Service } \\
\text { Specialists, Including Health Educators And }\end{array}$ & & & & & & \\
\hline II & $\begin{array}{l}\text { Community Health Workers } \\
\text { Miscellaneous Managers, Including Funeral } \\
\text { Service Managers And Postmasters And Mail }\end{array}$ & 2025 & 2025 & & 2 & 12 & 9 \\
\hline II & $\begin{array}{l}\text { Superintendents } \\
\text { Miscellaneous Managers, Including Funeral } \\
\text { Service Managers And Postmasters And Mail }\end{array}$ & 430 & 430 & & 162 & 204 & 146 \\
\hline II & $\begin{array}{l}\text { Superintendents } \\
\text { Miscellaneous Managers, Including Funeral } \\
\text { Service Managers And Postmasters And Mail }\end{array}$ & 430 & 400 & $x$ & 1 & 7 & 1 \\
\hline U & Superintendents & 430 & 325 & $x$ & 0 & 4 & 0 \\
\hline & Survey Researchers And Sociologists & 1860 & 1860 & & 4 & 7 & 5 \\
\hline II & $\begin{array}{l}\text { Miscellaneous Social Scientists, Including } \\
\text { Survey Researchers And Sociologists }\end{array}$ & 1860 & 1830 & $x$ & 0 & 1 & 0 \\
\hline II & Miscellaneous Social Scientists, Including & & & & & & \\
\hline II & $\begin{array}{l}\text { Survey Researchers And Sociologists } \\
\text { Network And Computer Systems }\end{array}$ & 1860 & 1815 & $x$ & 0 & 1 & 0 \\
\hline & Administrators & 1105 & 1105 & & 9 & 24 & 17 \\
\hline II & News Analysts, Reporters And & & & & & & \\
\hline & Correspondents & 2810 & 2810 & & 11 & 7 & 5 \\
\hline II & Nurse Anesthetists & 3256 & 3256 & & 0 & 2 & 3 \\
\hline II & Nurse Practitioners, And Nurse Midwives & 3258 & 3258 & & 3 & 5 & 5 \\
\hline II & Occupational Therapists & 3150 & 3150 & & 4 & 5 & 5 \\
\hline II & $\begin{array}{l}\text { Other Education, Training, And Library } \\
\text { Workers }\end{array}$ & 2550 & 2550 & & 6 & 6 & 8 \\
\hline
\end{tabular}




\begin{tabular}{|c|c|c|c|c|c|c|c|}
\hline Class & Occupational Title (ACS 2012) & $\begin{array}{l}\text { ACS } \\
2012\end{array}$ & $\begin{array}{r}\text { GSS } \\
\text { occ10 }\end{array}$ & $\begin{array}{l}\text { Recoded } \\
\text { to ACS }\end{array}$ & $\begin{array}{r}\text { GSS } \\
1994- \\
2000 \\
\end{array}$ & $\begin{array}{r}\text { GSS } \\
2002- \\
2008 \\
\end{array}$ & $\begin{array}{r}\text { GSS } \\
2010- \\
2016 \\
\end{array}$ \\
\hline \multirow[t]{2}{*}{ II } & Other Healthcare Practitioners And Technical & & & & & & \\
\hline & Occupations & 3540 & 3540 & & 8 & 9 & 6 \\
\hline \multirow[t]{2}{*}{ II } & Other Therapists, Including Exercise & & & & & & \\
\hline & Physiologists & 3245 & 3245 & & 8 & 11 & 8 \\
\hline \multirow[t]{2}{*}{ II } & Other Therapists, Including Exercise & & & & & & \\
\hline & Physiologists & 3245 & 3235 & $\mathrm{x}$ & 1 & 0 & 0 \\
\hline II & Personal Financial Advisors & 850 & 850 & & 17 & 24 & 18 \\
\hline II & Physical Therapists & 3160 & 3160 & & 13 & 11 & 7 \\
\hline II & Physician Assistants & 3110 & 3110 & & 0 & 5 & 5 \\
\hline II & Producers And Directors & 2710 & 2710 & & 10 & 21 & 15 \\
\hline II & Public Relations And Fundraising Managers & 60 & 60 & & 5 & 4 & 8 \\
\hline II & Public Relations Specialists & 2825 & 2825 & & 14 & 16 & 6 \\
\hline II & $\begin{array}{l}\text { Purchasing Agents, Except Wholesale, Retail, } \\
\text { And Farm Products }\end{array}$ & 530 & 530 & & 14 & 25 & 14 \\
\hline II & Purchasing Managers & 150 & 150 & & 15 & 7 & 15 \\
\hline II & Recreational Therapists & 3210 & 3210 & & 0 & 2 & 3 \\
\hline II & Registered Nurses & 3255 & 3255 & & 184 & 143 & 157 \\
\hline II & Sales Engineers & 4930 & 4930 & & 3 & 2 & 1 \\
\hline II & Secondary School Teachers & 2320 & 2320 & & 129 & 138 & 108 \\
\hline \multirow[t]{2}{*}{ II } & Securities, Commodities, And Financial & & & & & & \\
\hline & Services Sales Agents & 4820 & 4820 & & 21 & 24 & 9 \\
\hline II & Social And Community Service Managers & 420 & 420 & & 20 & 22 & 26 \\
\hline II & Social Workers & 2010 & 2010 & & 65 & 86 & 58 \\
\hline II & Special Education Teachers & 2330 & 2330 & & 32 & 29 & 31 \\
\hline II & Speech-Language Pathologists & 3230 & 3230 & & 5 & 11 & 9 \\
\hline \multirow[t]{2}{*}{ II } & Surveyors, Cartographers, And & & & & & & \\
\hline & Photogrammetrists & 1310 & 1310 & & 3 & 4 & 4 \\
\hline II & Technical Writers & 2840 & 2840 & & 9 & 3 & 3 \\
\hline II & Training And Development Managers & 137 & 137 & & 5 & 5 & 3 \\
\hline II & Training And Development Specialists & 650 & 650 & & 5 & 14 & 8 \\
\hline II & Writers And Authors & 2850 & 2850 & & 12 & 18 & 13 \\
\hline IIIa & Administrative Services Managers & 100 & 100 & & 10 & 7 & 5 \\
\hline IIIa & Advertising Sales Agents & 4800 & 4800 & & 14 & 15 & 9 \\
\hline \multirow[t]{2}{*}{ IIIa } & Air Traffic Controllers And Airfield & & & & & & \\
\hline & Operations Specialists & 9040 & 9040 & & 2 & 6 & 4 \\
\hline IIIa & Appraisers And Assessors Of Real Estate & 810 & 810 & & 4 & 2 & 4 \\
\hline IIIa & Bill And Account Collectors & 5100 & 5100 & & 10 & 13 & 11 \\
\hline IIIa & Billing And Posting Clerks & 5110 & 5110 & & 34 & 40 & 27 \\
\hline \multirow[t]{2}{*}{ IIIa } & Bookkeeping, Accounting, And Auditing & & & & & & \\
\hline & Clerks & 5120 & 5120 & & 131 & 114 & 72 \\
\hline IIIa & Brokerage Clerks & 5200 & 5200 & & 2 & 0 & 2 \\
\hline IIIa & Cargo And Freight Agents & 5500 & 5500 & & 1 & 4 & 0 \\
\hline IIIa & $\begin{array}{l}\text { Claims Adjusters, Appraisers, Examiners, And } \\
\text { Investigators }\end{array}$ & 540 & 540 & & 17 & 17 & 23 \\
\hline \multirow[t]{2}{*}{ IIIa } & Communications Equipment Operators, All & & & & & & \\
\hline & Other & 5030 & 5030 & & 0 & 1 & 1 \\
\hline
\end{tabular}




\begin{tabular}{|c|c|c|c|c|c|c|c|}
\hline Class & Occupational Title (ACS 2012) & $\begin{array}{l}\text { ACS } \\
2012 \\
\end{array}$ & $\begin{array}{r}\text { GSS } \\
\text { occ10 } \\
\end{array}$ & $\begin{array}{l}\text { Recoded } \\
\text { to ACS }\end{array}$ & $\begin{array}{c}\text { GSS } \\
1994- \\
2000 \\
\end{array}$ & $\begin{array}{r}\text { GSS } \\
2002- \\
2008 \\
\end{array}$ & $\begin{array}{r}\text { GSS } \\
2010- \\
2016 \\
\end{array}$ \\
\hline IIIa & Computer Operators & 5800 & 5800 & & 16 & 14 & 9 \\
\hline IIIa & Computer Support Specialists & 1050 & 1050 & & 22 & 44 & 34 \\
\hline IIIa & Correspondence Clerks And Order Clerks & 5350 & 5350 & & 8 & 8 & 2 \\
\hline IIIa & Correspondence Clerks And Order Clerks & 5350 & 5210 & $x$ & 0 & 1 & 0 \\
\hline IIIa & Court, Municipal, And License Clerks & 5220 & 5220 & & 9 & 6 & 6 \\
\hline IIIa & Credit Analysts & 830 & 830 & & 5 & 5 & 1 \\
\hline IIIa & Credit Authorizers, Checkers, And Clerks & 5230 & 5230 & & 9 & 5 & 1 \\
\hline IIIa & Credit Counselors And Loan Officers & 910 & 910 & & 22 & 23 & 13 \\
\hline IIIa & Customer Service Representatives & 5240 & 5240 & & 97 & 140 & 150 \\
\hline IIIa & Data Entry Keyers & 5810 & 5810 & & 38 & 45 & 14 \\
\hline IIIa & Dispatchers & 5520 & 5520 & & 14 & 31 & 11 \\
\hline \multirow[t]{2}{*}{ IIIa } & Eligibility Interviewers, Government & & & & & & \\
\hline & Programs & 5250 & 5250 & & 5 & 5 & 3 \\
\hline IIIa & File Clerks & 5260 & 5260 & & 11 & 12 & 6 \\
\hline IIIa & Financial Clerks, All Other & 5165 & 5165 & & 2 & 0 & 5 \\
\hline \multirow[t]{2}{*}{ IIIa } & First-Line Supervisors Of Office And & & & & & & \\
\hline & Administrative Support Workers & 5000 & 5000 & & 137 & 137 & 117 \\
\hline IIIa & Flight Attendants & 9050 & 9050 & & 8 & 4 & 7 \\
\hline IIIa & Hotel, Motel, And Resort Desk Clerks & 5300 & 5300 & & 5 & 13 & 9 \\
\hline \multirow[t]{2}{*}{ IIIa } & Human Resources Assistants, Except Payroll & & & & & & \\
\hline & And Timekeeping & 5360 & 5360 & & 10 & 10 & 11 \\
\hline IIIa & Human Resources Workers & 630 & 630 & & 26 & 32 & 37 \\
\hline IIIa & Information And Record Clerks, All Other & 5420 & 5420 & & 8 & 13 & 3 \\
\hline \multirow[t]{2}{*}{ IIIa } & Insurance Claims And Policy Processing & & & & & & \\
\hline & Clerks & 5840 & 5840 & & 28 & 15 & 18 \\
\hline IIIa & Insurance Sales Agents & 4810 & 4810 & & 19 & 36 & 28 \\
\hline IIIa & Interviewers, Except Eligibility And Loan & 5310 & 5310 & & 13 & 18 & 11 \\
\hline IIIa & Library Assistants, Clerical & 5320 & 5320 & & 20 & 12 & 3 \\
\hline IIIa & Library Technicians & 2440 & 2440 & & 2 & 3 & 3 \\
\hline \multirow[t]{2}{*}{ IIIa } & Licensed Practical And Licensed Vocational & & & & & & \\
\hline & Nurses & 3500 & 3500 & & 60 & 38 & 26 \\
\hline IIIa & Loan Interviewers And Clerks & 5330 & 5330 & & 18 & 11 & 9 \\
\hline IIIa & Lodging Managers & 340 & 340 & & 6 & 10 & 9 \\
\hline \multirow[t]{2}{*}{ IIIa } & Medical Records And Health Information & & & & & & \\
\hline & Technicians & 3510 & 3510 & & 6 & 5 & 10 \\
\hline IIIa & Meeting, Convention, And Event Planners & 725 & 725 & & 8 & 6 & 2 \\
\hline IIIa & Meter Readers, Utilities & 5530 & 5530 & & 3 & 5 & 0 \\
\hline IIIa & Miscellaneous Legal Support Workers & 2160 & 2160 & & 12 & 12 & 13 \\
\hline IIIa & $\begin{array}{l}\text { Miscellaneous Office And Administrative } \\
\text { Support Workers, Including Desktop }\end{array}$ & & & & & & \\
\hline \multirow{3}{*}{ IIIa } & Publishers & 5940 & 5940 & & 47 & 43 & 41 \\
\hline & Miscellaneous Office And Administrative & & & & & & \\
\hline & Publishers & 5940 & 5830 & $x$ & 1 & 1 & 0 \\
\hline IIIa & New Accounts Clerks & 5340 & 5340 & & 1 & 1 & 0 \\
\hline IIIa & Office Clerks, General & 5860 & 5860 & & 94 & 94 & 90 \\
\hline
\end{tabular}




\begin{tabular}{|c|c|c|c|c|c|c|c|}
\hline Class & Occupational Title (ACS 2012) & $\begin{array}{l}\text { ACS } \\
2012\end{array}$ & $\begin{array}{r}\text { GSS } \\
\text { occ10 }\end{array}$ & $\begin{array}{l}\text { Recoded } \\
\text { to ACS }\end{array}$ & $\begin{array}{r}\text { GSS } \\
1994- \\
2000\end{array}$ & $\begin{array}{r}\text { GSS } \\
2002- \\
2008\end{array}$ & $\begin{array}{r}\text { GSS } \\
2010- \\
2016\end{array}$ \\
\hline IIIa & Office Machine Operators, Except Computer & 5900 & 5900 & & 5 & 4 & 1 \\
\hline IIIa & Other Teachers And Instructors & 2340 & 2340 & & 34 & 33 & 33 \\
\hline IIIa & Paralegals And Legal Assistants & 2145 & 2145 & & 27 & 27 & 23 \\
\hline IIIa & Payroll And Timekeeping Clerks & 5140 & 5140 & & 22 & 24 & 8 \\
\hline IIIa & Postal Service Clerks & 5540 & 5540 & & 24 & 21 & 9 \\
\hline IIIa & Procurement Clerks & 5150 & 5150 & & 4 & 1 & 1 \\
\hline IIIa & Production, Planning, And Expediting Clerks & 5600 & 5600 & & 14 & 27 & 18 \\
\hline IIIa & Proofreaders And Copy Markers & 5910 & 5910 & & 1 & 1 & 0 \\
\hline IIIa & Radiation Therapists & 3200 & 3200 & & 0 & 1 & 1 \\
\hline IIIa & Real Estate Brokers And Sales Agents & 4920 & 4920 & & 27 & 29 & 21 \\
\hline \multirow[t]{2}{*}{ IIIa } & Reservation And Transportation Ticket Agents & & & & & & \\
\hline & And Travel Clerks & 5410 & 5410 & & 16 & 14 & 6 \\
\hline IIIa & Respiratory Therapists & 3220 & 3220 & & 7 & 5 & 4 \\
\hline IIIa & Sales And Related Workers, All Other & 4965 & 4965 & & 15 & 37 & 27 \\
\hline IIIa & Sales Representatives, Services, All Other & 4840 & 4840 & & 18 & 17 & 16 \\
\hline \multirow[t]{2}{*}{ IIIa } & Sales Representatives, Wholesale And & & & & & & \\
\hline & Manufacturing & 4850 & 4850 & & 66 & 114 & 46 \\
\hline IIIa & Secretaries And Administrative Assistants & 5700 & 5700 & & 448 & 388 & 226 \\
\hline IIIa & Social And Human Service Assistants & 2016 & 2016 & & 9 & 11 & 11 \\
\hline IIIa & Statistical Assistants & 5920 & 5920 & & 5 & 2 & 4 \\
\hline IIIa & Switchboard Operators, Including Answering & & & & & & \\
\hline \multirow{2}{*}{ IIIa } & $\begin{array}{l}\text { Service } \\
\text { Tax Examiners And Collectors, And Revenue }\end{array}$ & 5010 & 5010 & & 19 & 11 & 7 \\
\hline & Agents & 930 & 930 & & 6 & 3 & 8 \\
\hline IIIa & Tax Preparers & 940 & 940 & & 4 & 6 & 7 \\
\hline IIIa & Teacher Assistants & 2540 & 2540 & & 68 & 76 & 60 \\
\hline IIIa & Telephone Operators & 5020 & 5020 & & 20 & 12 & 7 \\
\hline IIIa & Tellers & 5160 & 5160 & & 36 & 40 & 23 \\
\hline IIIa & Tour And Travel Guides & 4540 & 4540 & & 2 & 1 & 2 \\
\hline IIIa & Travel Agents & 4830 & 4830 & & 14 & 6 & 4 \\
\hline IIIa & Web Developers & 1030 & 1030 & & 2 & 10 & 8 \\
\hline \multirow[t]{2}{*}{ IIIIa } & Weighers, Measurers, Checkers, And & & & & & & \\
\hline & Samplers, Recordkeeping & 5630 & 5630 & & 11 & 16 & 3 \\
\hline IIIa & Word Processors And Typists & 5820 & 5820 & & 32 & 29 & 9 \\
\hline $\mathrm{IIIb}$ & Animal Control Workers & 3900 & 3900 & & 1 & 1 & 0 \\
\hline \multirow[t]{2}{*}{$\mathrm{IIIlb}$} & Automotive And Watercraft Service & & & & & & \\
\hline & Attendants & 9360 & 9360 & & 9 & 7 & 4 \\
\hline $\mathrm{IIIb}$ & Baggage Porters, Bellhops, And Concierges & 4530 & 4530 & & 9 & 9 & 13 \\
\hline $\mathrm{IIIb}$ & Barbers & 4500 & 4500 & & 1 & 4 & 2 \\
\hline $\mathrm{IIIb}$ & Bartenders & 4040 & 4040 & & 32 & 32 & 29 \\
\hline IIIb & Bus Drivers & 9120 & 9120 & & 41 & 42 & 36 \\
\hline $\mathrm{IIIb}$ & Cashiers & 4720 & 4720 & & 186 & 184 & 152 \\
\hline $\mathrm{IIIb}$ & Childcare Workers & 4600 & 4600 & & 69 & 72 & 75 \\
\hline $\mathrm{IIIb}$ & $\begin{array}{l}\text { Coin, Vending, And Amusement Machine } \\
\text { Servicers And Repairers }\end{array}$ & 7510 & 7510 & & 2 & 3 & 3 \\
\hline
\end{tabular}




\begin{tabular}{|c|c|c|c|c|c|c|c|}
\hline Class & Occupational Title (ACS 2012) & $\begin{array}{l}\text { ACS } \\
2012\end{array}$ & $\begin{array}{r}\text { GSS } \\
\text { occ10 }\end{array}$ & $\begin{array}{l}\text { Recoded } \\
\text { to ACS }\end{array}$ & $\begin{array}{r}\text { GSS } \\
1994- \\
2000 \\
\end{array}$ & $\begin{array}{r}\text { GSS } \\
2002- \\
2008 \\
\end{array}$ & $\begin{array}{r}\text { GSS } \\
2010- \\
2016 \\
\end{array}$ \\
\hline \multirow[t]{2}{*}{ IIIb } & Combined Food Preparation And Serving & & & & & & \\
\hline & Workers, Including Fast Food & 4050 & 4050 & & 32 & 40 & 55 \\
\hline IIIb & Counter And Rental Clerks & 4740 & 4740 & & 17 & 8 & 10 \\
\hline \multirow[t]{2}{*}{ IIIb } & Counter Attendants, Cafeteria, Food & & & & & & \\
\hline & Concession, And Coffee Shop & 4060 & 4060 & & 13 & 10 & 13 \\
\hline $\mathrm{IIIb}$ & Couriers And Messengers & 5510 & 5510 & & 11 & 22 & 15 \\
\hline $\mathrm{IIIb}$ & Dental Assistants & 3640 & 3640 & & 12 & 19 & 23 \\
\hline \multirow[t]{2}{*}{$\mathrm{IIIb}$} & Door-To-Door Sales Workers, News And & & & & & & \\
\hline & Street Vendors, And Related Workers & 4950 & 4950 & & 16 & 8 & 4 \\
\hline $\mathrm{IIIb}$ & Embalmers And Funeral Attendants & 4460 & 4460 & & 0 & 2 & 0 \\
\hline $\mathrm{IIIb}$ & First-Line Supervisors Of Gaming Workers & 4300 & 4300 & & 3 & 0 & 2 \\
\hline \multirow[t]{2}{*}{$\mathrm{IIIb}$} & First-Line Supervisors Of Housekeeping And & & & & & & \\
\hline & Janitorial Workers & 4200 & 4200 & & 15 & 16 & 14 \\
\hline \multirow[t]{2}{*}{$\mathrm{IIIb}$} & First-Line Supervisors Of Personal Service & & & & & & \\
\hline & Workers & 4320 & 4320 & & 9 & 5 & 4 \\
\hline $\mathrm{IIIb}$ & First-Line Supervisors Of Retail Sales Workers & 4700 & 4700 & & 157 & 152 & 129 \\
\hline \multirow[t]{2}{*}{$\mathrm{IIIb}$} & First-Line Supervisors Of Food Preparation & & & & & & \\
\hline & And Serving Workers & 4010 & 4010 & & 54 & 54 & 43 \\
\hline $\mathrm{IIIb}$ & Food Servers, Nonrestaurant & 4120 & 4120 & & 11 & 7 & 10 \\
\hline $\mathrm{IIIb}$ & Food Service Managers & 310 & 310 & & 48 & 34 & 21 \\
\hline $\mathrm{IIIb}$ & Gaming Cage Workers & 5130 & 5130 & & 1 & 0 & 0 \\
\hline $\mathrm{IIIb}$ & Gaming Services Workers & 4400 & 4400 & & 7 & 7 & 2 \\
\hline IIIb & Hairdressers, Hairstylists, And Cosmetologists & 4510 & 4510 & & 36 & 33 & 27 \\
\hline $\mathrm{IIIlb}$ & $\begin{array}{l}\text { Healthcare Support Workers, All Other, } \\
\text { Including Medical Equipment Preparers }\end{array}$ & 3655 & 3655 & & 15 & 24 & 15 \\
\hline IIIb & $\begin{array}{l}\text { Hosts And Hostesses, Restaurant, Lounge, } \\
\text { And Coffee Shop }\end{array}$ & 4150 & 4150 & & 16 & 3 & 11 \\
\hline \multirow[t]{2}{*}{ IIIb } & Lifeguards And Other Recreational, And All & & & & & & \\
\hline & Other Protective Service Workers & 3955 & 3955 & & 8 & 6 & 4 \\
\hline $\mathrm{IIIb}$ & Massage Therapists & 3630 & 3630 & & 1 & 4 & 9 \\
\hline $\mathrm{IIIb}$ & Medical Assistants & 3645 & 3645 & & 26 & 25 & 23 \\
\hline IIIb & Medical Transcriptionists & 3646 & 3646 & & 7 & 9 & 2 \\
\hline \multirow[t]{2}{*}{$\mathrm{IIIb}$} & Miscellaneous Entertainment Attendants And & & & & & & \\
\hline & Related Workers & 4430 & 4430 & & 13 & 10 & 16 \\
\hline IIIb & $\begin{array}{l}\text { Miscellaneous Food Preparation And Serving } \\
\text { Related Workers, Including Dining Room And }\end{array}$ & & & & & & \\
\hline \multirow[t]{2}{*}{ IIIb } & $\begin{array}{l}\text { Cafeteria Attendants And Bartender Helpers } \\
\text { Miscellaneous Food Preparation And Serving } \\
\text { Related Workers, Including Dining Room And }\end{array}$ & 4130 & 4130 & & 14 & 26 & 21 \\
\hline & Cafeteria Attendants And Bartender Helpers & 4130 & 4160 & $\mathrm{x}$ & 0 & 2 & 2 \\
\hline $\mathrm{IIIb}$ & Miscellaneous Personal Appearance Workers & 4520 & 4520 & & 2 & 3 & 7 \\
\hline \multirow[t]{2}{*}{$\mathrm{IIIb}$} & $\begin{array}{l}\text { Miscellaneous Transportation Workers, } \\
\text { Including Bridge And Lock Tenders And }\end{array}$ & & & & & & \\
\hline & Traffic Technicians & 9420 & 9420 & & 1 & 2 & 0 \\
\hline \multirow[t]{2}{*}{$\mathrm{IIIb}$} & Models, Demonstrators, And Product & & & & & & \\
\hline & Promoters & 4900 & 4900 & & 6 & 8 & 4 \\
\hline \multirow[t]{2}{*}{$\mathrm{IIIb}$} & Morticians, Undertakers, And Funeral & & & & & & \\
\hline & Directors & 4465 & 4465 & & 1 & 1 & 3 \\
\hline
\end{tabular}




\begin{tabular}{|c|c|c|c|c|c|c|c|}
\hline Class & Occupational Title (ACS 2012) & $\begin{array}{l}\text { ACS } \\
2012 \\
\end{array}$ & $\begin{array}{r}\text { GSS } \\
\text { occ10 }\end{array}$ & $\begin{array}{l}\text { Recoded } \\
\text { to ACS }\end{array}$ & $\begin{array}{r}\text { GSS } \\
1994- \\
2000 \\
\end{array}$ & $\begin{array}{r}\text { GSS } \\
2002- \\
2008 \\
\end{array}$ & $\begin{array}{r}\text { GSS } \\
2010- \\
2016 \\
\end{array}$ \\
\hline IIIlb & Motion Picture Projectionists & 4410 & 4410 & & 0 & 1 & 2 \\
\hline $\mathrm{IIIb}$ & Nonfarm Animal Caretakers & 4350 & 4350 & & 8 & 8 & 4 \\
\hline $\mathrm{IIIb}$ & Nursing, Psychiatric, And Home Health Aides & 3600 & 3600 & & 227 & 264 & 224 \\
\hline $\mathrm{IIIb}$ & Occupational Therapy Assistants And Aides & 3610 & 3610 & & 1 & 3 & 0 \\
\hline $\mathrm{IIIb}$ & Parking Lot Attendants & 9350 & 9350 & & 7 & 1 & 5 \\
\hline $\mathrm{IIIb}$ & Parts Salespersons & 4750 & 4750 & & 9 & 10 & 14 \\
\hline IIIb & Personal Care Aides & 4610 & 4610 & & 35 & 34 & 53 \\
\hline $\mathrm{IIIb}$ & Personal Care And Service Workers, All Other & 4650 & 4650 & & 1 & 5 & 5 \\
\hline IIIlb & Pest Control Workers & 4240 & 4240 & & 4 & 2 & 3 \\
\hline $\mathrm{IIIb}$ & Pharmacy Aides & 3647 & 3647 & & 1 & 9 & 2 \\
\hline IIIb & Phlebotomists & 3649 & 3649 & & 3 & 6 & 12 \\
\hline $\mathrm{IIIb}$ & Physical Therapist Assistants And Aides & 3620 & 3620 & & 6 & 7 & 4 \\
\hline IIIb & Preschool And Kindergarten Teachers & 2300 & 2300 & & 33 & 50 & 53 \\
\hline IIIb & Receptionists And Information Clerks & 5400 & 5400 & & 86 & 69 & 54 \\
\hline IIIb & Recreation And Fitness Workers & 4620 & 4620 & & 7 & 24 & 23 \\
\hline $\mathrm{IIIb}$ & Religious Workers, All Other & 2060 & 2060 & & 1 & 6 & 5 \\
\hline IIIb & Residential Advisors & 4640 & 4640 & & 4 & 4 & 0 \\
\hline $\mathrm{IIIb}$ & Retail Salespersons & 4760 & 4760 & & 236 & 216 & 171 \\
\hline IIIlb & $\begin{array}{l}\text { Security Guards And Gaming Surveillance } \\
\text { Officers }\end{array}$ & 3930 & 3930 & & 43 & 48 & 30 \\
\hline IIIb & Telemarketers & 4940 & 4940 & & 20 & 13 & 8 \\
\hline $\mathrm{IIIb}$ & $\begin{array}{l}\text { Transportation Attendants, Except Flight } \\
\text { Attendants }\end{array}$ & 9415 & 9415 & & 1 & 1 & 2 \\
\hline $\mathrm{IIIb}$ & Transportation Security Screeners & 3945 & 3945 & & 1 & 4 & 0 \\
\hline IIIb & Ushers, Lobby Attendants, And Ticket Takers & 4420 & 4420 & & 3 & 1 & 2 \\
\hline $\mathrm{IIIb}$ & $\begin{array}{l}\text { Veterinary Assistants And Laboratory Animal } \\
\text { Caretakers }\end{array}$ & 3648 & 3648 & & 5 & 2 & 2 \\
\hline $\mathrm{IIIb}$ & Waiters And Waitresses & 4110 & 4110 & & 176 & 185 & 131 \\
\hline \multirow[t]{2}{*}{ IIIlb } & Wholesale And Retail Buyers, Except Farm & & & & & & \\
\hline & Products & 520 & 520 & & 14 & 12 & 5 \\
\hline IVab & Actors & 2700 & 2700 & & 1 & 0 & 1 \\
\hline IVab & Advertising Sales Agents & 4800 & 4800 & & 1 & 3 & 1 \\
\hline IVab & Agricultural Inspectors & 6010 & 6010 & & 0 & 0 & 1 \\
\hline IVab & Aircraft Mechanics And Service Technicians & 7140 & 7140 & & 1 & 0 & 0 \\
\hline IVab & Animal Trainers & 4340 & 4340 & & 1 & 1 & 0 \\
\hline IVab & Announcers & 2800 & 2800 & & 2 & 0 & 0 \\
\hline IVab & Appraisers And Assessors Of Real Estate & 810 & 810 & & 6 & 4 & 3 \\
\hline IVab & Artists And Related Workers & 2600 & 2600 & & 20 & 21 & 8 \\
\hline \multirow[t]{2}{*}{ IVab } & Athletes, Coaches, Umpires, And Related & & & & & & \\
\hline & Workers & 2720 & 2720 & & 1 & 1 & 4 \\
\hline IVab & Automotive Body And Related Repairers & 7150 & 7150 & & 5 & 2 & 3 \\
\hline IVab & Automotive Glass Installers And Repairers & 7160 & 7160 & & 0 & 0 & 2 \\
\hline \multirow[t]{2}{*}{ IVab } & Automotive Service Technicians And & & & & & & \\
\hline & Mechanics & 7200 & 7200 & & 19 & 21 & 12 \\
\hline IVab & Bakers & 7800 & 7800 & & 2 & 4 & 0 \\
\hline
\end{tabular}




\begin{tabular}{|c|c|c|c|c|c|c|c|}
\hline Class & Occupational Title (ACS 2012) & $\begin{array}{l}\text { ACS } \\
2012 \\
\end{array}$ & $\begin{array}{r}\text { GSS } \\
\text { occ10 }\end{array}$ & $\begin{array}{l}\text { Recoded } \\
\text { to ACS }\end{array}$ & $\begin{array}{r}\text { GSS } \\
1994- \\
2000 \\
\end{array}$ & $\begin{array}{r}\text { GSS } \\
2002- \\
2008 \\
\end{array}$ & $\begin{array}{r}\text { GSS } \\
2010- \\
2016 \\
\end{array}$ \\
\hline IVab & Barbers & 4500 & 4500 & & 2 & 3 & 5 \\
\hline IVab & Bartenders & 4040 & 4040 & & 2 & 4 & 4 \\
\hline IVab & Billing And Posting Clerks & 5110 & 5110 & & 0 & 1 & 0 \\
\hline IVab & $\begin{array}{l}\text { Bookkeeping, Accounting, And Auditing } \\
\text { Clerks } \\
\text { Brickmasons, Blockmasons, Stonemasons, And }\end{array}$ & 5120 & 5120 & & 18 & 13 & 8 \\
\hline IVab & $\begin{array}{l}\text { Reinforcing Iron And Rebar Workers } \\
\text { Broadcast And Sound Engineering } \\
\text { Technicians And Radio Operators, And Media } \\
\text { And Communication Equipment Workers, All }\end{array}$ & 6220 & 6220 & & 2 & 3 & 4 \\
\hline IVab & $\begin{array}{l}\text { Other } \\
\text { Bus And Truck Mechanics And Diesel Engine }\end{array}$ & 2900 & 2900 & & 1 & 1 & 1 \\
\hline & Specialists & 7210 & 7210 & & 1 & 1 & 1 \\
\hline IVab & Bus Drivers & 9120 & 9120 & & 4 & 0 & 1 \\
\hline IVab & $\begin{array}{l}\text { Butchers And Other Meat, Poultry, And Fish } \\
\text { Processing Workers } \\
\text { Buyers And Purchasing Agents, Farm }\end{array}$ & 7810 & 7810 & & 1 & 0 & 0 \\
\hline & Products & 510 & 510 & & 0 & 1 & 2 \\
\hline IVab & Cabinetmakers And Bench Carpenters & 8500 & 8500 & & 4 & 8 & 3 \\
\hline IVab & Cargo And Freight Agents & 5500 & 5500 & & 1 & 0 & 0 \\
\hline IVab & Carpenters & 6230 & 6230 & & 33 & 34 & 24 \\
\hline IVab & $\begin{array}{l}\text { Carpet, Floor, And Tile Installers And } \\
\text { Finishers }\end{array}$ & 6240 & 6240 & & 7 & 6 & 2 \\
\hline IVab & Cashiers & 4720 & 4720 & & 2 & 3 & 1 \\
\hline IVab & $\begin{array}{l}\text { Cement Masons, Concrete Finishers, And } \\
\text { Terrazzo Workers }\end{array}$ & 6250 & 6250 & & 2 & 1 & 0 \\
\hline IVab & Chefs And Head Cooks & 4000 & 4000 & & 0 & 2 & 3 \\
\hline IVab & Childcare Workers & 4600 & 4600 & & 56 & 57 & 30 \\
\hline IVab & $\begin{array}{l}\text { Claims Adjusters, Appraisers, Examiners, And } \\
\text { Investigators }\end{array}$ & 540 & 540 & & 2 & 1 & 0 \\
\hline IVab & Cleaners Of Vehicles And Equipment & 9610 & 9610 & & 2 & 4 & 0 \\
\hline IVab & $\begin{array}{l}\text { Clinical Laboratory Technologists And } \\
\text { Technicians }\end{array}$ & 3300 & 3300 & & 1 & 0 & 0 \\
\hline IVab & Computer Support Specialists & 1050 & 1050 & & 1 & 2 & 0 \\
\hline IVab & $\begin{array}{l}\text { Computer, Automated Teller, And Office } \\
\text { Machine Repairers }\end{array}$ & 7010 & 7010 & & 1 & 4 & 2 \\
\hline IVab & Construction And Building Inspectors & 6660 & 6660 & & 0 & 0 & 1 \\
\hline IVab & $\begin{array}{l}\text { Construction Equipment Operators, Except } \\
\text { Paving, Surfacing, And Tamping Equipment }\end{array}$ & & & & & & \\
\hline & Operators & 6320 & 6320 & & 1 & 3 & 4 \\
\hline IVab & Construction Laborers & 6260 & 6260 & & 11 & 26 & 28 \\
\hline IVab & Construction Managers & 220 & 220 & & 36 & 44 & 29 \\
\hline IVab & Cooks & 4020 & 4020 & & 4 & 1 & 1 \\
\hline IVab & Cost Estimators & 600 & 600 & & 0 & 0 & 1 \\
\hline IVab & Counter And Rental Clerks & 4740 & 4740 & & 0 & 1 & 2 \\
\hline IVab & $\begin{array}{l}\text { Counter Attendants, Cafeteria, Food } \\
\text { Concession, And Coffee Shop }\end{array}$ & 4060 & 4060 & & 1 & 0 & 0 \\
\hline
\end{tabular}




\begin{tabular}{|c|c|c|c|c|c|c|c|}
\hline Class & Occupational Title (ACS 2012) & $\begin{array}{l}\text { ACS } \\
2012 \\
\end{array}$ & $\begin{array}{r}\text { GSS } \\
\text { occ10 }\end{array}$ & $\begin{array}{l}\text { Recoded } \\
\text { to ACS }\end{array}$ & $\begin{array}{r}\text { GSS } \\
1994- \\
2000 \\
\end{array}$ & $\begin{array}{r}\text { GSS } \\
2002- \\
2008 \\
\end{array}$ & $\begin{array}{r}\text { GSS } \\
2010- \\
2016 \\
\end{array}$ \\
\hline IVab & Couriers And Messengers & 5510 & 5510 & & 1 & 2 & 2 \\
\hline IVab & Crane And Tower Operators & 9510 & 9510 & & 0 & 0 & 1 \\
\hline IVab & Credit Counselors And Loan Officers & 910 & 910 & & 2 & 0 & 2 \\
\hline IVab & $\begin{array}{l}\text { Crushing, Grinding, Polishing, Mixing, And } \\
\text { Blending Workers }\end{array}$ & 8650 & 8650 & & 1 & 0 & 0 \\
\hline IVab & Customer Service Representatives & 5240 & 5240 & & 1 & 1 & 1 \\
\hline IVab & Dancers And Choreographers & 2740 & 2740 & & 4 & 0 & 1 \\
\hline IVab & Dental Hygienists & 3310 & 3310 & & 0 & 2 & 1 \\
\hline IVab & $\begin{array}{l}\text { Derrick, Rotary Drill, And Service Unit } \\
\text { Operators, And Roustabouts, Oil, Gas, And } \\
\text { Mining }\end{array}$ & 6800 & 6800 & & 0 & 1 & 0 \\
\hline IVab & Designers & 2630 & 2630 & & 20 & 17 & 15 \\
\hline IVab & Detectives And Criminal Investigators & 3820 & 3820 & & 1 & 2 & 0 \\
\hline IVab & $\begin{array}{l}\text { Diagnostic Related Technologists And } \\
\text { Technicians }\end{array}$ & 3320 & 3320 & & 0 & 0 & 1 \\
\hline IVab & Dishwashers & 4140 & 4140 & & 0 & 0 & 1 \\
\hline IVab & Dispatchers & 5520 & 5520 & & 1 & 0 & 0 \\
\hline IVab & $\begin{array}{l}\text { Door-To-Door Sales Workers, News And } \\
\text { Street Vendors, And Related Workers }\end{array}$ & 4950 & 4950 & & 2 & 4 & 4 \\
\hline IVab & Drafters & 1540 & 1540 & & 0 & 1 & 1 \\
\hline IVab & $\begin{array}{l}\text { Dredge, Excavating, And Loading Machine } \\
\text { Operators }\end{array}$ & 9520 & 9520 & & 0 & 3 & 0 \\
\hline IVab & Driver/Sales Workers And Truck Drivers & 9130 & 9130 & & 21 & 29 & 23 \\
\hline IVab & $\begin{array}{l}\text { Drywall Installers, Ceiling Tile Installers, And } \\
\text { Tapers }\end{array}$ & 6330 & 6330 & & 3 & 4 & 0 \\
\hline IVab & $\begin{array}{l}\text { Electric Motor, Power Tool, And Related } \\
\text { Repairers }\end{array}$ & 7040 & 7040 & & 0 & 0 & 1 \\
\hline IVab & $\begin{array}{l}\text { Electrical, Electronics, And Electromechanical } \\
\text { Assemblers }\end{array}$ & 7720 & 7720 & & 0 & 2 & 0 \\
\hline IVab & Electricians & 6355 & 6355 & & 3 & 4 & 4 \\
\hline IVab & $\begin{array}{l}\text { Electronic Home Entertainment Equipment } \\
\text { Installers And Repairers }\end{array}$ & 7120 & 7120 & & 1 & 1 & 2 \\
\hline IVab & $\begin{array}{l}\text { Entertainers And Performers, Sports And } \\
\text { Related Workers, All Other }\end{array}$ & 2760 & 2760 & & 1 & 1 & 2 \\
\hline IVab & Fence Erectors & 6710 & 6710 & & 0 & 1 & 0 \\
\hline IVab & First-Line Supervisors Of Construction Trades & & & & & & \\
\hline & And Extraction Workers & 6200 & 6200 & & 21 & 23 & 10 \\
\hline IVab & First-Line Supervisors Of Correctional Officers & 3700 & 3700 & & 1 & 0 & 0 \\
\hline IVab & $\begin{array}{l}\text { First-Line Supervisors Of Farming, Fishing, } \\
\text { And Forestry Workers }\end{array}$ & 6005 & 6005 & & 1 & 0 & 0 \\
\hline IVab & $\begin{array}{l}\text { First-Line Supervisors Of Housekeeping And } \\
\text { Janitorial Workers }\end{array}$ & 4200 & 4200 & & 8 & 6 & 2 \\
\hline IVab & First-Line Supervisors Of Landscaping, Lawn & & & & & & \\
\hline & Service, And Groundskeeping Workers & 4210 & 4210 & & 6 & 7 & 8 \\
\hline IVab & First-Line Supervisors Of Mechanics, & & & & & & \\
\hline & Installers, And Repairers & 7000 & 7000 & & 0 & 2 & 0 \\
\hline IVab & First-Line Supervisors Of Non-Retail Sales & & & & & & \\
\hline & Workers & 4710 & 4710 & & 19 & 10 & 12 \\
\hline
\end{tabular}




\begin{tabular}{|c|c|c|c|c|c|c|c|}
\hline Class & Occupational Title (ACS 2012) & $\begin{array}{l}\text { ACS } \\
2012\end{array}$ & $\begin{array}{r}\text { GSS } \\
\text { occ10 }\end{array}$ & $\begin{array}{l}\text { Recoded } \\
\text { to ACS }\end{array}$ & $\begin{array}{r}\text { GSS } \\
1994- \\
2000\end{array}$ & $\begin{array}{r}\text { GSS } \\
2002- \\
2008\end{array}$ & $\begin{array}{r}\text { GSS } \\
2010- \\
2016\end{array}$ \\
\hline IVab & $\begin{array}{l}\text { First-Line Supervisors Of Office And } \\
\text { Administrative Support Workers }\end{array}$ & 5000 & 5000 & & 6 & 2 & 5 \\
\hline IVab & $\begin{array}{l}\text { First-Line Supervisors Of Personal Service } \\
\text { Workers }\end{array}$ & 4320 & 4320 & & 4 & 4 & 1 \\
\hline IVab & $\begin{array}{l}\text { First-Line Supervisors Of Production And } \\
\text { Operating Workers }\end{array}$ & 7700 & 7700 & & 8 & 5 & 1 \\
\hline IVab & First-Line Supervisors Of Retail Sales Workers & 4700 & 4700 & & 76 & 64 & 38 \\
\hline IVab & $\begin{array}{l}\text { First-Line Supervisors Of Food Preparation } \\
\text { And Serving Workers }\end{array}$ & 4010 & 4010 & & 4 & 7 & 2 \\
\hline IVab & Fishing And Hunting Workers & 6100 & 6100 & & 2 & 8 & 3 \\
\hline IVab & Food Preparation Workers & 4030 & 4030 & & 1 & 0 & 0 \\
\hline IVab & Food Servers, Nonrestaurant & 4120 & 4120 & & 1 & 0 & 0 \\
\hline IVab & Food Service Managers & 310 & 310 & & 28 & 20 & 19 \\
\hline IVab & Furniture Finishers & 8510 & 8510 & & 0 & 0 & 1 \\
\hline IVab & Gaming Managers & 330 & 330 & & 0 & 1 & 0 \\
\hline IVab & Grounds Maintenance Workers & 4250 & 4250 & & 13 & 14 & 20 \\
\hline IVab & Hairdressers, Hairstylists, And Cosmetologists & 4510 & 4510 & & 46 & 37 & 40 \\
\hline IVab & $\begin{array}{l}\text { Health Practitioner Support Technologists } \\
\text { And Technicians }\end{array}$ & 3420 & 3420 & & 1 & 0 & 1 \\
\hline IVab & $\begin{array}{l}\text { Healthcare Support Workers, All Other, } \\
\text { Including Medical Equipment Preparers }\end{array}$ & 3655 & 3655 & & 1 & 0 & 0 \\
\hline IVab & $\begin{array}{l}\text { Heating, Air Conditioning, And Refrigeration } \\
\text { Mechanics And Installers }\end{array}$ & 7315 & 7315 & & 1 & 6 & 1 \\
\hline IVab & $\begin{array}{l}\text { Heavy Vehicle And Mobile Equipment Service } \\
\text { Technicians And Mechanics }\end{array}$ & 7220 & 7220 & & 1 & 0 & 0 \\
\hline IVab & $\begin{array}{l}\text { Helpers-Installation, Maintenance, And Repair } \\
\text { Workers }\end{array}$ & 7610 & 7610 & & 1 & 0 & 0 \\
\hline IVab & Highway Maintenance Workers & 6730 & 6730 & & 0 & 1 & 0 \\
\hline IVab & Home Appliance Repairers & 7320 & 7320 & & 0 & 0 & 1 \\
\hline IVab & $\begin{array}{l}\text { Human Resources Assistants, Except Payroll } \\
\text { And Timekeeping }\end{array}$ & 5360 & 5360 & & 1 & 0 & 0 \\
\hline IVab & Human Resources Workers & 630 & 630 & & 1 & 2 & 5 \\
\hline IVab & $\begin{array}{l}\text { Industrial And Refractory Machinery } \\
\text { Mechanics }\end{array}$ & 7330 & 7330 & & 0 & 1 & 1 \\
\hline IVab & Industrial Truck And Tractor Operators & 9600 & 9600 & & 0 & 2 & 0 \\
\hline IVab & $\begin{array}{l}\text { Inspectors, Testers, Sorters, Samplers, And } \\
\text { Weighers }\end{array}$ & 8740 & 8740 & & 0 & 2 & 1 \\
\hline IVab & Insulation Workers & 6400 & 6400 & & 0 & 1 & 0 \\
\hline IVab & Insurance Sales Agents & 4810 & 4810 & & 10 & 10 & 10 \\
\hline IVab & Interviewers, Except Eligibility And Loan & 5310 & 5310 & & 0 & 0 & 1 \\
\hline IVab & Janitors And Building Cleaners & 4220 & 4220 & & 6 & 7 & 12 \\
\hline IVab & $\begin{array}{l}\text { Jewelers And Precious Stone And Metal } \\
\text { Workers }\end{array}$ & 8750 & 8750 & & 0 & 0 & 1 \\
\hline IVab & $\begin{array}{l}\text { Laborers And Freight, Stock, And Material } \\
\text { Movers, Hand }\end{array}$ & 9620 & 9620 & & 1 & 6 & 5 \\
\hline IVab & Laundry And Dry-Cleaning Workers & 8300 & 8300 & & 3 & 1 & 3 \\
\hline
\end{tabular}




\begin{tabular}{|c|c|c|c|c|c|c|c|}
\hline Class & Occupational Title (ACS 2012) & $\begin{array}{l}\text { ACS } \\
2012 \\
\end{array}$ & $\begin{array}{r}\text { GSS } \\
\text { occ10 }\end{array}$ & $\begin{array}{l}\text { Recoded } \\
\text { to ACS }\end{array}$ & $\begin{array}{r}\text { GSS } \\
1994- \\
2000 \\
\end{array}$ & $\begin{array}{r}\text { GSS } \\
2002- \\
2008 \\
\end{array}$ & $\begin{array}{r}\text { GSS } \\
2010- \\
2016 \\
\end{array}$ \\
\hline \multirow[t]{2}{*}{ IVab } & Licensed Practical And Licensed Vocational & & & & & & \\
\hline & Nurses & 3500 & 3500 & & 0 & 2 & 0 \\
\hline IVab & Loan Interviewers And Clerks & 5330 & 5330 & & 0 & 1 & 0 \\
\hline IVab & Locksmiths And Safe Repairers & 7540 & 7540 & & 0 & 3 & 0 \\
\hline IVab & Lodging Managers & 340 & 340 & & 2 & 5 & 1 \\
\hline IVab & Logging Workers & 6130 & 6130 & & 1 & 8 & 2 \\
\hline \multirow[t]{2}{*}{ IVab } & Machine Tool Cutting Setters, Operators, And & & & & & & \\
\hline & Tenders, Metal And Plastic & 7950 & 7950 & & 1 & 0 & 0 \\
\hline \multirow[t]{2}{*}{ IVab } & Machine Tool Cutting Setters, Operators, And & & & & & & \\
\hline & Tenders, Metal And Plastic & 7950 & 8010 & $\mathrm{x}$ & 0 & 1 & 0 \\
\hline IVab & Machinists & 8030 & 8030 & & 1 & 2 & 1 \\
\hline IVab & Maids And Housekeeping Cleaners & 4230 & 4230 & & 46 & 50 & 41 \\
\hline IVab & Maintenance And Repair Workers, General & 7340 & 7340 & & 1 & 0 & 2 \\
\hline IVab & Massage Therapists & 3630 & 3630 & & 5 & 9 & 8 \\
\hline IVab & Medical Assistants & 3645 & 3645 & & 0 & 1 & 0 \\
\hline \multirow[t]{2}{*}{ IVab } & Medical, Dental, And Ophthalmic Laboratory & & & & & & \\
\hline & Technicians & 8760 & 8760 & & 4 & 2 & 0 \\
\hline IVab & Meeting, Convention, And Event Planners & 725 & 725 & & 1 & 1 & 0 \\
\hline IVab & $\begin{array}{l}\text { Miscellaneous Agricultural Workers, } \\
\text { Including Animal Breeders }\end{array}$ & 6050 & 6050 & & 1 & 3 & 6 \\
\hline \multirow[t]{2}{*}{ IVab } & Miscellaneous Agricultural Workers, & & & & & & \\
\hline & Including Animal Breeders & 6050 & 6020 & $\mathrm{x}$ & 1 & 0 & 0 \\
\hline IVab & Miscellaneous Assemblers And Fabricators & 7750 & 7750 & & 3 & 4 & 2 \\
\hline \multirow[t]{2}{*}{ IVab } & Miscellaneous Extraction Workers, Including & & & & & & \\
\hline & Roof Bolters And Helpers & 6940 & 6930 & $\mathrm{x}$ & 1 & 0 & 0 \\
\hline \multirow[t]{2}{*}{ IVab } & $\begin{array}{l}\text { Miscellaneous Food Preparation And Serving } \\
\text { Related Workers, Including Dining Room And }\end{array}$ & & & & & & \\
\hline & Cafeteria Attendants And Bartender Helpers & 4130 & 4130 & & 1 & 0 & 0 \\
\hline \multirow[t]{2}{*}{ IVab } & Miscellaneous Health Technologists And & & & & & & \\
\hline & Technicians & 3535 & 3535 & & 1 & 0 & 0 \\
\hline \multirow[t]{2}{*}{ IVab } & $\begin{array}{l}\text { Miscellaneous Installation, Maintenance, And } \\
\text { Repair Workers, Including Wind Turbine }\end{array}$ & & & & & & \\
\hline & Service Technicians & 7630 & 7630 & & 0 & 2 & 1 \\
\hline \multirow[t]{2}{*}{ IVab } & $\begin{array}{l}\text { Miscellaneous Installation, Maintenance, And } \\
\text { Repair Workers, Including Wind Turbine }\end{array}$ & & & & & & \\
\hline & Service Technicians & 7630 & 7550 & $\mathrm{x}$ & 2 & 0 & 0 \\
\hline IVab & Miscellaneous Legal Support Workers & 2160 & 2160 & & 3 & 3 & 4 \\
\hline \multirow[t]{2}{*}{ IVab } & $\begin{array}{l}\text { Miscellaneous Life, Physical, And Social } \\
\text { Science Technicians, Including Social Science }\end{array}$ & & & & & & \\
\hline & Research Assistants & 1965 & 1965 & & 1 & 0 & 0 \\
\hline \multirow[t]{2}{*}{ IVab } & $\begin{array}{l}\text { Miscellaneous Material Moving Workers, } \\
\text { Including Mine Shuttle Car Operators, And }\end{array}$ & & & & & & \\
\hline & Tank Car, Truck, And Ship Loaders & 9750 & 9750 & & 0 & 0 & 1 \\
\hline \multirow[t]{2}{*}{ IVab } & Miscellaneous Media And Communication & & & & & & \\
\hline & Workers & 2860 & 2860 & & 2 & 3 & 3 \\
\hline \multirow[t]{2}{*}{ IVab } & $\begin{array}{l}\text { Miscellaneous Office And Administrative } \\
\text { Support Workers, Including Desktop }\end{array}$ & & & & & & \\
\hline & Publishers & 5940 & 5940 & & 1 & 2 & 0 \\
\hline
\end{tabular}




\begin{tabular}{|c|c|c|c|c|c|c|c|}
\hline Class & Occupational Title (ACS 2012) & $\begin{array}{l}\text { ACS } \\
2012 \\
\end{array}$ & $\begin{array}{r}\text { GSS } \\
\text { occ10 }\end{array}$ & $\begin{array}{l}\text { Recoded } \\
\text { to ACS }\end{array}$ & $\begin{array}{r}\text { GSS } \\
1994- \\
2000 \\
\end{array}$ & $\begin{array}{r}\text { GSS } \\
2002- \\
2008 \\
\end{array}$ & $\begin{array}{r}\text { GSS } \\
2010- \\
2016 \\
\end{array}$ \\
\hline IVab & Miscellaneous Personal Appearance Workers & 4520 & 4520 & & 1 & 7 & 4 \\
\hline IVab & Miscellaneous Plant And System Operators & 8630 & 8630 & & 1 & 0 & 0 \\
\hline IVab & $\begin{array}{l}\text { Miscellaneous Production Workers, Including } \\
\text { Semiconductor Processors }\end{array}$ & 8965 & 8965 & & 1 & 3 & 1 \\
\hline IVab & $\begin{array}{l}\text { Miscellaneous Production Workers, Including } \\
\text { Semiconductor Processors }\end{array}$ & 8965 & 8900 & $x$ & 1 & 0 & 0 \\
\hline IVab & $\begin{array}{l}\text { Miscellaneous Textile, Apparel, And } \\
\text { Furnishings Workers, Except Upholsterers }\end{array}$ & 8460 & 8060 & & & & 0 \\
\hline IVab & Miscellaneous Vehicle And Mobile Equipment & & & & & & \\
\hline IVab & $\begin{array}{l}\text { Mechanics, Installers, And Repairers } \\
\text { Miscellaneous Woodworkers, Including }\end{array}$ & 7260 & 7260 & & 0 & 1 & 0 \\
\hline IVab & $\begin{array}{l}\text { Model Makers And Patternmakers } \\
\text { Model Makers, Patternmakers, And Molding }\end{array}$ & 8550 & 8550 & & 1 & 1 & 0 \\
\hline & Machine Setters, Metal And Plastic & 8100 & 8100 & & 1 & 0 & 0 \\
\hline IVab & $\begin{array}{l}\text { Morticians, Undertakers, And Funeral } \\
\text { Directors }\end{array}$ & 4465 & 4465 & & 0 & 0 & 1 \\
\hline IVab & Motion Picture Projectionists & 4410 & 4410 & & 0 & 0 & 1 \\
\hline IVab & Musicians, Singers, And Related Workers & 2750 & 2750 & & 15 & 8 & 8 \\
\hline IVab & Nonfarm Animal Caretakers & 4350 & 4350 & & 1 & 3 & 4 \\
\hline IVab & Nursing, Psychiatric, And Home Health Aides & 3600 & 3600 & & 6 & 10 & 6 \\
\hline IVab & Office Clerks, General & 5860 & 5860 & & 3 & 1 & 2 \\
\hline IVab & Opticians, Dispensing & 3520 & 3520 & & 1 & 0 & 0 \\
\hline IVab & Other Teachers And Instructors & 2340 & 2340 & & 13 & 12 & 14 \\
\hline IVab & Packaging And Filling Machine Operators & & & & & & \\
\hline & And Tenders & 8800 & 8800 & & 0 & 1 & 0 \\
\hline IVab & Packers And Packagers, Hand & 9640 & 9640 & & 0 & 1 & 0 \\
\hline IVab & Painters And Paperhangers & 6420 & 6420 & & 13 & 12 & 15 \\
\hline IVab & Painters And Paperhangers & 6420 & 6430 & $x$ & 3 & 1 & 0 \\
\hline IVab & Painting Workers & 8810 & 8810 & & 2 & 1 & 1 \\
\hline IVab & Paralegals And Legal Assistants & 2145 & 2145 & & 0 & 1 & 2 \\
\hline IVab & Parts Salespersons & 4750 & 4750 & & 2 & 0 & 1 \\
\hline IVab & Paving, Surfacing, And Tamping Equipment & & & & & & \\
\hline & Operators & 6300 & 6300 & & 0 & 0 & 1 \\
\hline IVab & Payroll And Timekeeping Clerks & 5140 & 5140 & & 1 & 0 & 1 \\
\hline IVab & Personal Care Aides & 4610 & 4610 & & 8 & 12 & 18 \\
\hline IVab & Personal Care And Service Workers, All Other & 4650 & 4650 & & 0 & 0 & 4 \\
\hline IVab & Pest Control Workers & 4240 & 4240 & & 1 & 1 & 1 \\
\hline IVab & Photographers & 2910 & 2910 & & 5 & 12 & 4 \\
\hline IVab & Pipelayers, Plumbers, Pipefitters, And & & & & & & \\
\hline & Steamfitters & 6440 & 6440 & & 8 & 10 & 8 \\
\hline IVab & Police Officers & 3850 & 3850 & & 2 & 0 & 0 \\
\hline IVab & Postal Service Clerks & 5540 & 5540 & & 1 & 0 & 0 \\
\hline IVab & Precision Instrument And Equipment & & & & & & \\
\hline & Repairers & 7430 & 7430 & & 0 & 2 & 0 \\
\hline IVab & Preschool And Kindergarten Teachers & 2300 & 2300 & & 1 & 1 & 0 \\
\hline
\end{tabular}




\begin{tabular}{|c|c|c|c|c|c|c|c|}
\hline Class & Occupational Title (ACS 2012) & $\begin{array}{l}\text { ACS } \\
2012\end{array}$ & $\begin{array}{r}\text { GSS } \\
\text { occ10 }\end{array}$ & $\begin{array}{l}\text { Recoded } \\
\text { to ACS }\end{array}$ & $\begin{array}{r}\text { GSS } \\
1994- \\
2000 \\
\end{array}$ & $\begin{array}{r}\text { GSS } \\
2002- \\
2008 \\
\end{array}$ & $\begin{array}{r}\text { GSS } \\
2010- \\
2016 \\
\end{array}$ \\
\hline \multirow[t]{2}{*}{$\mathrm{IVab}$} & Pressers, Textile, Garment, And Related & & & & & & \\
\hline & Materials & 8310 & 8310 & & 1 & 0 & 0 \\
\hline IVab & Printing Press Operators & 8255 & 8255 & & 3 & 4 & 4 \\
\hline IVab & Private Detectives And Investigators & 3910 & 3910 & & 1 & 2 & 0 \\
\hline IVab & Production, Planning, And Expediting Clerks & 5600 & 5600 & & 0 & 0 & 1 \\
\hline \multirow[t]{2}{*}{ IVab } & Property, Real Estate, And Community & & & & & & \\
\hline & Association Managers & 410 & 410 & & 10 & 10 & 8 \\
\hline IVab & Pumping Station Operators & 9650 & 9650 & & 0 & 2 & 0 \\
\hline IVab & $\begin{array}{l}\text { Radio And Telecommunications Equipment } \\
\text { Installers And Repairers }\end{array}$ & 7020 & 7020 & & 0 & 1 & 1 \\
\hline IVab & Real Estate Brokers And Sales Agents & 4920 & 4920 & & 41 & 47 & 30 \\
\hline IVab & Receptionists And Information Clerks & 5400 & 5400 & & 2 & 0 & 0 \\
\hline IVab & Recreation And Fitness Workers & 4620 & 4620 & & 3 & 6 & 1 \\
\hline IVab & Refuse And Recyclable Material Collectors & 9720 & 9720 & & 1 & 2 & 1 \\
\hline IVab & Religious Workers, All Other & 2060 & 2060 & & 0 & 0 & 1 \\
\hline IVab & Retail Salespersons & 4760 & 4760 & & 26 & 33 & 20 \\
\hline IVab & Riggers & 7560 & 7560 & & 1 & 0 & 0 \\
\hline IVab & Roofers & 6515 & 6515 & & 0 & 0 & 4 \\
\hline IVab & Sales And Related Workers, All Other & 4965 & 4965 & & 0 & 4 & 0 \\
\hline IVab & Sales Representatives, Services, All Other & 4840 & 4840 & & 8 & 2 & 4 \\
\hline \multirow[t]{2}{*}{ IVab } & Sales Representatives, Wholesale And & & & & & & \\
\hline & Manufacturing & 4850 & 4850 & & 22 & 15 & 14 \\
\hline IVab & Secretaries And Administrative Assistants & 5700 & 5700 & & 14 & 14 & 6 \\
\hline \multirow[t]{2}{*}{ IVab } & Security Guards And Gaming Surveillance & & & & & & \\
\hline & Officers & 3930 & 3930 & & 0 & 2 & 1 \\
\hline IVab & Sewing Machine Operators & 8320 & 8320 & & 3 & 1 & 0 \\
\hline IVab & Sheet Metal Workers & 6520 & 6520 & & 1 & 1 & 0 \\
\hline IVab & Shipping, Receiving, And Traffic Clerks & 5610 & 5610 & & 0 & 1 & 0 \\
\hline IVab & Shoe And Leather Workers & 8330 & 8330 & & 1 & 2 & 0 \\
\hline IVab & Stationary Engineers And Boiler Operators & 8610 & 8610 & & 1 & 1 & 0 \\
\hline IVab & Stock Clerks And Order Fillers & 5620 & 5620 & & 2 & 1 & 0 \\
\hline IVab & Structural Iron And Steel Workers & 6530 & 6530 & & 0 & 0 & 1 \\
\hline IVab & Structural Metal Fabricators And Fitters & 7740 & 7740 & & 1 & 0 & 2 \\
\hline \multirow[t]{2}{*}{ IVab } & Supervisors Of Transportation And Material & & & & & & \\
\hline & Moving Workers & 9000 & 9000 & & 0 & 4 & 1 \\
\hline IVab & Surveying And Mapping Technicians & 1560 & 1560 & & 2 & 1 & 0 \\
\hline IVab & Tailors, Dressmakers, And Sewers & 8350 & 8350 & & 6 & 11 & 4 \\
\hline IVab & Tax Preparers & 940 & 940 & & 4 & 2 & 5 \\
\hline IVab & Taxi Drivers And Chauffeurs & 9140 & 9140 & & 5 & 6 & 8 \\
\hline IVab & Teacher Assistants & 2540 & 2540 & & 1 & 0 & 1 \\
\hline \multirow[t]{2}{*}{ IVab } & Telecommunications Line Installers And & & & & & & \\
\hline & Repairers & 7420 & 7420 & & 0 & 1 & 1 \\
\hline IVab & Telemarketers & 4940 & 4940 & & 0 & 1 & 0 \\
\hline \multirow[t]{2}{*}{ IVab } & Television, Video, And Motion Picture Camera & & & & & & \\
\hline & Operators And Editors & 2920 & 2920 & & 3 & 2 & 0 \\
\hline
\end{tabular}




\begin{tabular}{|c|c|c|c|c|c|c|c|}
\hline Class & Occupational Title (ACS 2012) & $\begin{array}{l}\text { ACS } \\
2012 \\
\end{array}$ & $\begin{array}{r}\text { GSS } \\
\text { occ10 }\end{array}$ & $\begin{array}{l}\text { Recoded } \\
\text { to ACS }\end{array}$ & $\begin{array}{r}\text { GSS } \\
1994- \\
2000 \\
\end{array}$ & $\begin{array}{r}\text { GSS } \\
2002- \\
2008 \\
\end{array}$ & $\begin{array}{r}\text { GSS } \\
2010- \\
2016 \\
\end{array}$ \\
\hline IVab & Tellers & 5160 & 5160 & & 1 & 0 & 0 \\
\hline \multirow[t]{2}{*}{ IVab } & Textile Knitting And Weaving Machine & & & & & & \\
\hline & Setters, Operators, And Tenders & 8410 & 8410 & & 0 & 1 & 0 \\
\hline \multirow[t]{2}{*}{ IVab } & Textile Winding, Twisting, And Drawing Out & & & & & & \\
\hline & Machine Setters, Operators, And Tenders & 8420 & 8420 & & 1 & 0 & 0 \\
\hline IVab & Travel Agents & 4830 & 4830 & & 3 & 2 & 3 \\
\hline IVab & Upholsterers & 8450 & 8450 & & 4 & 3 & 0 \\
\hline IVab & Waiters And Waitresses & 4110 & 4110 & & 3 & 2 & 1 \\
\hline IVab & $\begin{array}{l}\text { Water And Wastewater Treatment Plant And } \\
\text { System Operators }\end{array}$ & 8620 & 8620 & & 1 & 0 & 0 \\
\hline IVab & Web Developers & 1030 & 1030 & & 0 & 3 & 2 \\
\hline IVab & Welding, Soldering, And Brazing Workers & 8140 & 8140 & & 4 & 4 & 7 \\
\hline \multirow[t]{2}{*}{ IVab } & Wholesale And Retail Buyers, Except Farm & & & & & & \\
\hline & Products & 520 & 520 & & 3 & 3 & 1 \\
\hline IVab & $\begin{array}{l}\text { Woodworking Machine Setters, Operators, } \\
\text { And Tenders, Except Sawing }\end{array}$ & 8540 & 8540 & & 0 & 1 & 1 \\
\hline IVab & Word Processors And Typists & 5820 & 5820 & & 1 & 0 & 0 \\
\hline \multirow[t]{2}{*}{ IVc } & Farmers, Ranchers, And Other Agricultural & & & & & & \\
\hline & Managers & 205 & 205 & & 69 & 63 & 25 \\
\hline V & Actors & 2700 & 2700 & & 3 & 2 & 0 \\
\hline $\mathrm{V}$ & Agricultural And Food Science Technicians & 1900 & 1900 & & 5 & 3 & 5 \\
\hline $\mathrm{V}$ & Announcers & 2800 & 2800 & & 2 & 2 & 5 \\
\hline $\mathrm{V}$ & Artists And Related Workers & 2600 & 2600 & & 8 & 10 & 6 \\
\hline \multirow[t]{2}{*}{$\mathrm{V}$} & Athletes, Coaches, Umpires, And Related & & & & & & \\
\hline & Workers & 2720 & 2720 & & 8 & 10 & 12 \\
\hline V & Avionics Technicians & 7030 & 7030 & & 3 & 1 & 1 \\
\hline $\mathrm{V}$ & Bailiffs, Correctional Officers, And Jailers & 3800 & 3800 & & 22 & 24 & 28 \\
\hline $\mathrm{V}$ & Biological Technicians & 1910 & 1910 & & 1 & 3 & 3 \\
\hline \multirow[t]{2}{*}{$\mathrm{V}$} & $\begin{array}{l}\text { Broadcast And Sound Engineering } \\
\text { Technicians And Radio Operators, And Media } \\
\text { And Communication Equipment Workers, All }\end{array}$ & & & & & & \\
\hline & Other & 2900 & 2900 & & 3 & 6 & 8 \\
\hline \multirow[t]{2}{*}{$\mathrm{V}$} & Buyers And Purchasing Agents, Farm & & & & & & \\
\hline & Products & 510 & 510 & & 3 & 3 & 0 \\
\hline $\mathrm{V}$ & Chefs And Head Cooks & 4000 & 4000 & & 15 & 23 & 25 \\
\hline V & Chemical Technicians & 1920 & 1920 & & 11 & 6 & 6 \\
\hline $\mathrm{V}$ & Clinical Laboratory Technologists And & & & & & & \\
\hline & $\begin{array}{l}\text { Technicians } \\
\text { Computer Control Programmers And }\end{array}$ & 3300 & 3300 & & 20 & 27 & 26 \\
\hline $\mathrm{V}$ & Operators & 7900 & 7900 & & 2 & 0 & 1 \\
\hline $\mathrm{V}$ & Construction And Building Inspectors & 6660 & 6660 & & 6 & 5 & 2 \\
\hline $\mathrm{V}$ & Construction Managers & 220 & 220 & & 16 & 32 & 24 \\
\hline $\mathrm{V}$ & Cost Estimators & 600 & 600 & & 6 & 3 & 2 \\
\hline $\mathrm{V}$ & Dancers And Choreographers & 2740 & 2740 & & 1 & 1 & 0 \\
\hline $\mathrm{V}$ & Dental Hygienists & 3310 & 3310 & & 9 & 5 & 4 \\
\hline $\mathrm{V}$ & Designers & 2630 & 2630 & & 31 & 47 & 28 \\
\hline
\end{tabular}




\begin{tabular}{|c|c|c|c|c|c|c|c|}
\hline Class & Occupational Title (ACS 2012) & $\begin{array}{l}\text { ACS } \\
2012\end{array}$ & $\begin{array}{r}\text { GSS } \\
\text { occ10 }\end{array}$ & $\begin{array}{l}\text { Recoded } \\
\text { to ACS }\end{array}$ & $\begin{array}{r}\text { GSS } \\
1994- \\
2000\end{array}$ & $\begin{array}{r}\text { GSS } \\
2002- \\
2008\end{array}$ & $\begin{array}{r}\text { GSS } \\
2010- \\
2016\end{array}$ \\
\hline $\mathrm{V}$ & Detectives And Criminal Investigators & 3820 & 3820 & & 8 & 10 & 10 \\
\hline \multirow[t]{2}{*}{$\mathrm{V}$} & Diagnostic Related Technologists And & & & & & & \\
\hline & Technicians & 3320 & 3320 & & 19 & 21 & 26 \\
\hline $\mathrm{V}$ & Drafters & 1540 & 1540 & & 13 & 19 & 5 \\
\hline $\mathrm{V}$ & Electric Motor, Power Tool, And Related & & & & & & \\
\hline \multirow[t]{2}{*}{$\mathrm{V}$} & $\begin{array}{l}\text { Repairers } \\
\text { Electrical And Electronics Repairers, }\end{array}$ & 7040 & 7040 & & 8 & 4 & 4 \\
\hline & $\begin{array}{l}\text { Transportation Equipment, And Industrial } \\
\text { And Utility }\end{array}$ & 7100 & 7100 & & 2 & 2 & 0 \\
\hline \multirow[t]{2}{*}{$\mathrm{V}$} & Electronic Equipment Installers And & & & & & & \\
\hline & Repairers, Motor Vehicles & 7110 & 7110 & & 5 & 2 & 2 \\
\hline \multirow[t]{2}{*}{$\mathrm{V}$} & Electronic Home Entertainment Equipment & & & & & & \\
\hline & Installers And Repairers & 7120 & 7120 & & 4 & 2 & 1 \\
\hline $\mathrm{V}$ & Elevator Installers And Repairers & 6700 & 6700 & & 1 & 2 & 0 \\
\hline \multirow[t]{2}{*}{$\mathrm{V}$} & Emergency Medical Technicians And & & & & & & \\
\hline & Paramedics & 3400 & 3400 & & 7 & 14 & 10 \\
\hline $\mathrm{V}$ & Engineering Technicians, Except Drafters & 1550 & 1550 & & 54 & 38 & 25 \\
\hline \multirow[t]{2}{*}{$\mathrm{V}$} & Entertainers And Performers, Sports And & & & & & & \\
\hline & Related Workers, All Other & 2760 & 2760 & & 2 & 4 & 0 \\
\hline $\mathrm{V}$ & Fire Inspectors & 3750 & 3750 & & 1 & 5 & 1 \\
\hline $\mathrm{V}$ & Firefighters & 3740 & 3740 & & 15 & 29 & 16 \\
\hline \multirow[t]{2}{*}{$\mathrm{V}$} & First-Line Supervisors Of Construction Trades & & & & & & \\
\hline & And Extraction Workers & 6200 & 6200 & & 41 & 55 & 21 \\
\hline $\mathrm{V}$ & First-Line Supervisors Of Correctional Officers & 3700 & 3700 & & 4 & 8 & 5 \\
\hline \multirow[t]{2}{*}{$\mathrm{V}$} & First-Line Supervisors Of Landscaping, Lawn & & & & & & \\
\hline & Service, And Groundskeeping Workers & 4210 & 4210 & & 8 & 7 & 7 \\
\hline \multirow[t]{2}{*}{$\mathrm{V}$} & First-Line Supervisors Of Mechanics, & & & & & & \\
\hline & Installers, And Repairers & 7000 & 7000 & & 33 & 42 & 13 \\
\hline \multirow[t]{2}{*}{$\mathrm{V}$} & First-Line Supervisors Of Non-Retail Sales & & & & & & \\
\hline & Workers & 4710 & 4710 & & 38 & 52 & 27 \\
\hline \multirow[t]{2}{*}{$\mathrm{V}$} & First-Line Supervisors Of Production And & & & & & & \\
\hline & Operating Workers & 7700 & 7700 & & 100 & 82 & 41 \\
\hline \multirow[t]{2}{*}{$\mathrm{V}$} & First-Line Supervisors Of Protective Service & & & & & & \\
\hline & Workers, All Other & 3730 & 3730 & & 10 & 11 & 6 \\
\hline \multirow[t]{2}{*}{$\mathrm{V}$} & First-Line Supervisors Of Fire Fighting And & & & & & & \\
\hline & Prevention Workers & 3720 & 3720 & & 4 & 3 & 2 \\
\hline \multirow[t]{2}{*}{$\mathrm{V}$} & First-Line Supervisors Of Police And & & & & & & \\
\hline & Detectives & 3710 & 3710 & & 7 & 3 & 8 \\
\hline $\mathrm{V}$ & Gaming Managers & 330 & 330 & & 2 & 2 & 3 \\
\hline $\mathrm{V}$ & $\begin{array}{l}\text { Geological And Petroleum Technicians, And } \\
\text { Nuclear Technicians }\end{array}$ & 1930 & 1930 & & 2 & 3 & 2 \\
\hline \multirow[t]{2}{*}{$\mathrm{V}$} & Geological And Petroleum Technicians, And & & & & & & \\
\hline & Nuclear Technicians & 1930 & 1940 & $\mathrm{x}$ & 1 & 0 & 0 \\
\hline \multirow[t]{2}{*}{$\mathrm{V}$} & Health Practitioner Support Technologists & & & & & & \\
\hline & And Technicians & 3420 & 3420 & & 28 & 45 & 43 \\
\hline \multirow[t]{2}{*}{$\mathrm{V}$} & Miscellaneous Health Technologists And & & & & & & \\
\hline & Technicians & 3535 & 3535 & & 4 & 7 & 7 \\
\hline $\mathrm{V}$ & Miscellaneous Law Enforcement Workers & 3840 & 3840 & & 1 & 0 & 1 \\
\hline
\end{tabular}




\begin{tabular}{|c|c|c|c|c|c|c|c|}
\hline Class & Occupational Title (ACS 2012) & $\begin{array}{l}\text { ACS } \\
2012 \\
\end{array}$ & $\begin{array}{r}\text { GSS } \\
\text { occ10 }\end{array}$ & $\begin{array}{l}\text { Recoded } \\
\text { to ACS }\end{array}$ & $\begin{array}{r}\text { GSS } \\
1994- \\
2000 \\
\end{array}$ & $\begin{array}{r}\text { GSS } \\
2002- \\
2008 \\
\end{array}$ & $\begin{array}{r}\text { GSS } \\
2010- \\
2016 \\
\end{array}$ \\
\hline $\mathrm{V}$ & Miscellaneous Law Enforcement Workers & 3840 & 3830 & $\mathrm{x}$ & 1 & 0 & 0 \\
\hline $\mathrm{V}$ & $\begin{array}{l}\text { Miscellaneous Life, Physical, And Social } \\
\text { Science Technicians, Including Social Science }\end{array}$ & & & & & & \\
\hline \multirow[t]{2}{*}{ V } & $\begin{array}{l}\text { Research Assistants } \\
\text { Miscellaneous Life, Physical, And Social } \\
\text { Science Technicians, Including Social Science }\end{array}$ & 1965 & 1965 & & 22 & 25 & 8 \\
\hline & Research Assistants & 1965 & 1950 & $\mathrm{x}$ & 0 & 1 & 1 \\
\hline \multirow[t]{2}{*}{$\mathrm{V}$} & Miscellaneous Media And Communication & & & & & & \\
\hline & Workers & 2860 & 2860 & & 4 & 4 & 5 \\
\hline $\mathrm{V}$ & Musicians, Singers, And Related Workers & 2750 & 2750 & & 15 & 8 & 5 \\
\hline $\mathrm{V}$ & Opticians, Dispensing & 3520 & 3520 & & 2 & 4 & 7 \\
\hline $\mathrm{V}$ & Photographers & 2910 & 2910 & & 2 & 6 & 8 \\
\hline $\mathrm{V}$ & Police Officers & 3850 & 3850 & & 51 & 50 & 42 \\
\hline $\mathrm{V}$ & Private Detectives And Investigators & 3910 & 3910 & & 6 & 7 & 5 \\
\hline $\mathrm{V}$ & $\begin{array}{l}\text { Probation Officers And Correctional } \\
\text { Treatment Specialists }\end{array}$ & 2015 & 2015 & & 3 & 7 & 4 \\
\hline $\mathrm{V}$ & $\begin{array}{l}\text { Property, Real Estate, And Community } \\
\text { Association Managers }\end{array}$ & 410 & 410 & & 32 & 26 & 26 \\
\hline \multirow[t]{2}{*}{$\mathrm{V}$} & Radio And Telecommunications Equipment & & & & & & \\
\hline & Installers And Repairers & 7020 & 7020 & & 8 & 19 & 10 \\
\hline $\mathrm{V}$ & Railroad Conductors And Yardmasters & 9240 & 9240 & & 8 & 2 & 5 \\
\hline $\mathrm{V}$ & Ship And Boat Captains And Operators & 9310 & 9310 & & 2 & 1 & 1 \\
\hline $\mathrm{V}$ & $\begin{array}{l}\text { Supervisors Of Transportation And Material } \\
\text { Moving Workers }\end{array}$ & 9000 & 9000 & & 20 & 21 & 14 \\
\hline $\mathrm{V}$ & Surveying And Mapping Technicians & 1560 & 1560 & & 1 & 5 & 6 \\
\hline \multirow[t]{2}{*}{$\mathrm{V}$} & Telecommunications Line Installers And & & & & & & \\
\hline & $\begin{array}{l}\text { Repairers } \\
\text { Television, Video, And Motion Picture Camera }\end{array}$ & 7420 & 7420 & & 13 & 14 & 7 \\
\hline $\mathrm{V}$ & $\begin{array}{l}\text { I elevision, Video, And Motion Picture Camera } \\
\text { Operators And Editors }\end{array}$ & 2920 & 2920 & & 2 & 1 & 2 \\
\hline $\mathrm{V}$ & Transportation Inspectors & 9410 & 9410 & & 7 & 4 & 4 \\
\hline \multirow[t]{2}{*}{$\mathrm{V}$} & Transportation, Storage, And Distribution & & & & & & \\
\hline & Managers & 160 & 160 & & 25 & 16 & 16 \\
\hline VI & Aircraft Mechanics And Service Technicians & 7140 & 7140 & & 16 & 10 & 13 \\
\hline \multirow[t]{2}{*}{ VI } & Automotive Service Technicians And & & & & & & \\
\hline & Mechanics & 7200 & 7200 & & 53 & 52 & 37 \\
\hline VI & Boilermakers & 6210 & 6210 & & 2 & 1 & 2 \\
\hline \multirow[t]{2}{*}{ VI } & Brickmasons, Blockmasons, Stonemasons, And & & & & & & \\
\hline & Reinforcing Iron And Rebar Workers & 6220 & 6220 & & 12 & 10 & 11 \\
\hline \multirow[t]{2}{*}{ VI } & Brickmasons, Blockmasons, Stonemasons, And & & & & & & \\
\hline & Reinforcing Iron And Rebar Workers & 6220 & 6500 & $x$ & 2 & 1 & 0 \\
\hline \multirow[t]{2}{*}{ VI } & Bus And Truck Mechanics And Diesel Engine & & & & & & \\
\hline & Specialists & 7210 & 7210 & & 24 & 28 & 11 \\
\hline VI & Cabinetmakers And Bench Carpenters & 8500 & 8500 & & 1 & 6 & 1 \\
\hline VI & Carpenters & 6230 & 6230 & & 65 & 90 & 41 \\
\hline \multirow[t]{2}{*}{ VI } & Carpet, Floor, And Tile Installers And & & & & & & \\
\hline & Finishers & 6240 & 6240 & & 13 & 6 & 10 \\
\hline \multirow[t]{2}{*}{ VI } & Computer, Automated Teller, And Office & & & & & & \\
\hline & Machine Repairers & 7010 & 7010 & & 14 & 19 & 9 \\
\hline
\end{tabular}




\begin{tabular}{|c|c|c|c|c|c|c|c|}
\hline Class & Occupational Title (ACS 2012) & $\begin{array}{l}\text { ACS } \\
2012\end{array}$ & $\begin{array}{r}\text { GSS } \\
\text { occ10 }\end{array}$ & $\begin{array}{l}\text { Recoded } \\
\text { to ACS }\end{array}$ & $\begin{array}{r}\text { GSS } \\
1994- \\
2000\end{array}$ & $\begin{array}{r}\text { GSS } \\
2002- \\
2008\end{array}$ & $\begin{array}{r}\text { GSS } \\
2010- \\
2016\end{array}$ \\
\hline \multirow[t]{3}{*}{ VI } & Construction Equipment Operators, Except & & & & & & \\
\hline & Paving, Surfacing, And Tamping Equipment & & & & & & \\
\hline & Operators & 6320 & 6320 & & 33 & 31 & 22 \\
\hline VI & Control And Valve Installers And Repairers & 7300 & 7300 & & 0 & 3 & 0 \\
\hline VI & Crane And Tower Operators & 9510 & 9510 & & 7 & 7 & 6 \\
\hline \multirow[t]{2}{*}{ VI } & Drywall Installers, Ceiling Tile Installers, And & & & & & & \\
\hline & Tapers & 6330 & 6330 & & 5 & 8 & 6 \\
\hline VI & Electrical Power-Line Installers And Repairers & 7410 & 7410 & & 11 & 4 & 10 \\
\hline VI & Electricians & 6355 & 6355 & & 59 & 42 & 35 \\
\hline VI & Etchers And Engravers & 8910 & 8910 & & 1 & 0 & 0 \\
\hline \multirow[t]{2}{*}{ VI } & Explosives Workers, Ordnance Handling & & & & & & \\
\hline & Experts, And Blasters & 6830 & 6830 & & 3 & 1 & 3 \\
\hline VI & Forest And Conservation Workers & 6120 & 6120 & & 0 & 2 & 1 \\
\hline VI & Hazardous Materials Removal Workers & 6720 & 6720 & & 5 & 1 & 3 \\
\hline \multirow[t]{2}{*}{ VI } & Heating, Air Conditioning, And Refrigeration & & & & & & \\
\hline & Mechanics And Installers & 7315 & 7315 & & 22 & 24 & 20 \\
\hline \multirow[t]{2}{*}{ VI } & Heavy Vehicle And Mobile Equipment Service & & & & & & \\
\hline & Technicians And Mechanics & 7220 & 7220 & & 10 & 20 & 6 \\
\hline VI & Home Appliance Repairers & 7320 & 7320 & & 7 & 2 & 5 \\
\hline \multirow[t]{2}{*}{ VI } & Industrial And Refractory Machinery & & & & & & \\
\hline & Mechanics & 7330 & 7330 & & 26 & 28 & 8 \\
\hline VI & Insulation Workers & 6400 & 6400 & & 1 & 3 & 4 \\
\hline \multirow[t]{2}{*}{ VI } & Jewelers And Precious Stone And Metal & & & & & & \\
\hline & Workers & 8750 & 8750 & & 2 & 3 & 3 \\
\hline VI & Locksmiths And Safe Repairers & 7540 & 7540 & & 2 & 1 & 2 \\
\hline VI & Locomotive Engineers And Operators & 9200 & 9200 & & 5 & 4 & 3 \\
\hline VI & Machinists & 8030 & 8030 & & 27 & 31 & 23 \\
\hline VI & Maintenance And Repair Workers, General & 7340 & 7340 & & 14 & 14 & 15 \\
\hline VI & Maintenance Workers, Machinery & 7350 & 7350 & & 2 & 4 & 2 \\
\hline \multirow[t]{2}{*}{ VI } & Medical, Dental, And Ophthalmic Laboratory & & & & & & \\
\hline & Technicians & 8760 & 8760 & & 4 & 5 & 5 \\
\hline VI & Millwrights & 7360 & 7360 & & 5 & 9 & 10 \\
\hline VI & Miscellaneous Installation, Maintenance, And & & & & & & \\
\hline \multirow{3}{*}{ VI } & $\begin{array}{l}\text { Repair Workers, Including Wind Turbine } \\
\text { Service Technicians }\end{array}$ & 7630 & 7630 & & 12 & 17 & 9 \\
\hline & $\begin{array}{l}\text { Miscellaneous Installation, Maintenance, And } \\
\text { Repair Workers, Including Wind Turbine }\end{array}$ & & & & & & \\
\hline & Service Technicians & 7630 & 7550 & $x$ & 1 & 0 & 0 \\
\hline VI & Miscellaneous Plant And System Operators & 8630 & 8630 & & 2 & 6 & 5 \\
\hline \multirow[t]{2}{*}{ VI } & Miscellaneous Woodworkers, Including & & & & & & \\
\hline & Model Makers And Patternmakers & 8550 & 8550 & & 3 & 2 & 0 \\
\hline \multirow[t]{2}{*}{ VI } & Miscellaneous Woodworkers, Including & & & & & & \\
\hline & Model Makers And Patternmakers & 8550 & 8520 & $x$ & 1 & 0 & 0 \\
\hline VI & Model Makers, Patternmakers, And Molding & & & & & & \\
\hline \multirow{3}{*}{ VI } & Machine Setters, Metal And Plastic & 8100 & 8100 & & 15 & 11 & 6 \\
\hline & Model Makers, Patternmakers, And Molding & & & & & & \\
\hline & Machine Setters, Metal And Plastic & 8100 & 8060 & $x$ & 1 & 1 & 0 \\
\hline
\end{tabular}




\begin{tabular}{|c|c|c|c|c|c|c|c|}
\hline Class & Occupational Title (ACS 2012) & $\begin{array}{l}\text { ACS } \\
2012 \\
\end{array}$ & $\begin{array}{r}\text { GSS } \\
\text { occ10 } \\
\end{array}$ & $\begin{array}{l}\text { Recoded } \\
\text { to ACS }\end{array}$ & $\begin{array}{r}\text { GSS } \\
1994- \\
2000 \\
\end{array}$ & $\begin{array}{r}\text { GSS } \\
2002- \\
2008 \\
\end{array}$ & $\begin{array}{r}\text { GSS } \\
2010- \\
2016 \\
\end{array}$ \\
\hline \multirow[t]{2}{*}{ VI } & Molders, Shapers, And Casters, Except Metal & & & & & & \\
\hline & And Plastic & 8920 & 8920 & & 2 & 7 & 0 \\
\hline VI & Painters And Paperhangers & 6420 & 6420 & & 17 & 20 & 16 \\
\hline VI & Painters And Paperhangers & 6420 & 6430 & $\mathrm{x}$ & 1 & 0 & 0 \\
\hline \multirow{2}{*}{ VI } & Pipelayers, Plumbers, Pipefitters, And & & & & & & \\
\hline & Steamfitters & 6440 & 6440 & & 57 & 36 & 26 \\
\hline \multirow[t]{2}{*}{ VI } & Power Plant Operators, Distributors, And & & & & & & \\
\hline & Dispatchers & 8600 & 8600 & & 3 & 3 & 0 \\
\hline \multirow[t]{2}{*}{ VI } & Precision Instrument And Equipment & & & & & & \\
\hline & Repairers & 7430 & 7430 & & 8 & 8 & 1 \\
\hline VI & Prepress Technicians And Workers & 8250 & 8250 & & 10 & 8 & 6 \\
\hline VI & Printing Press Operators & 8255 & 8255 & & 30 & 26 & 7 \\
\hline \multirow[t]{2}{*}{ VI } & Sailors And Marine Oilers, And Ship & & & & & & \\
\hline & Engineers & 9300 & 9300 & & 2 & 2 & 2 \\
\hline VI & Security And Fire Alarm Systems Installers & 7130 & 7130 & & 3 & 1 & 3 \\
\hline VI & Sheet Metal Workers & 6520 & 6520 & & 7 & 13 & 7 \\
\hline VI & Small Engine Mechanics & 7240 & 7240 & & 2 & 5 & 6 \\
\hline VI & Stationary Engineers And Boiler Operators & 8610 & 8610 & & 13 & 7 & 12 \\
\hline VI & Structural Iron And Steel Workers & 6530 & 6530 & & 8 & 5 & 8 \\
\hline VI & Structural Metal Fabricators And Fitters & 7740 & 7740 & & 6 & 5 & 3 \\
\hline \multirow[t]{2}{*}{ VI } & Subway, Streetcar, And Other Rail & & & & & & \\
\hline & Transportation Workers & 9260 & 9260 & & 3 & 1 & 1 \\
\hline \multirow[t]{2}{*}{ VI } & Subway, Streetcar, And Other Rail & & & & & & \\
\hline & Transportation Workers & 9260 & 9230 & $\mathrm{x}$ & 1 & 2 & 0 \\
\hline VI & Tailors, Dressmakers, And Sewers & 8350 & 8350 & & 10 & 4 & 8 \\
\hline VI & Tool And Die Makers & 8130 & 8130 & & 10 & 10 & 9 \\
\hline \multirow[t]{2}{*}{ VI } & Water And Wastewater Treatment Plant And & & & & & & \\
\hline & System Operators & 8620 & 8620 & & 8 & 3 & 6 \\
\hline \multirow[t]{2}{*}{ VIIa } & Adhesive Bonding Machine Operators And & & & & & & \\
\hline & Tenders & 8850 & 8850 & & 3 & 2 & 0 \\
\hline \multirow[t]{2}{*}{ VIIa } & Aircraft Structure, Surfaces, Rigging, And & & & & & & \\
\hline & Systems Assemblers & 7710 & 7710 & & 4 & 5 & 1 \\
\hline VIIa & Automotive Body And Related Repairers & 7150 & 7150 & & 15 & 13 & 10 \\
\hline VIIa & Automotive Glass Installers And Repairers & 7160 & 7160 & & 3 & 2 & 0 \\
\hline VIIa & Bakers & 7800 & 7800 & & 19 & 10 & 13 \\
\hline \multirow[t]{2}{*}{ VIIa } & Butchers And Other Meat, Poultry, And Fish & & & & & & \\
\hline & Processing Workers & 7810 & 7810 & & 17 & 23 & 12 \\
\hline \multirow[t]{2}{*}{ VIIa } & Cement Masons, Concrete Finishers, And & & & & & & \\
\hline & Terrazzo Workers & 6250 & 6250 & & 8 & 4 & 5 \\
\hline \multirow[t]{2}{*}{ VIIa } & Chemical Processing Machine Setters, & & & & & & \\
\hline & Operators, And Tenders & 8640 & 8640 & & 12 & 8 & 6 \\
\hline VIIa & Cleaners Of Vehicles And Equipment & 9610 & 9610 & & 24 & 12 & 9 \\
\hline VIIa & Construction Laborers & 6260 & 6260 & & 58 & 91 & 76 \\
\hline \multirow[t]{2}{*}{ VIIa } & Conveyor Operators And Tenders, And Hoist & & & & & & \\
\hline & And Winch Operators & 9560 & 9500 & $\mathrm{x}$ & 0 & 1 & 0 \\
\hline VIIa & Cooks & 4020 & 4020 & & 117 & 152 & 115 \\
\hline VIIa & Crossing Guards & 3940 & 3940 & & 6 & 6 & 3 \\
\hline
\end{tabular}




\begin{tabular}{|c|c|c|c|c|c|c|c|}
\hline Class & Occupational Title (ACS 2012) & $\begin{array}{l}\text { ACS } \\
2012 \\
\end{array}$ & $\begin{array}{r}\text { GSS } \\
\text { occ10 }\end{array}$ & $\begin{array}{l}\text { Recoded } \\
\text { to ACS }\end{array}$ & $\begin{array}{r}\text { GSS } \\
1994- \\
2000 \\
\end{array}$ & $\begin{array}{r}\text { GSS } \\
2002- \\
2008 \\
\end{array}$ & $\begin{array}{r}\text { GSS } \\
2010- \\
2016 \\
\end{array}$ \\
\hline \multirow[t]{2}{*}{ VIIa } & Crushing, Grinding, Polishing, Mixing, And & & & & & & \\
\hline & Blending Workers & 8650 & 8650 & & 6 & 5 & 6 \\
\hline VIIa & Cutting Workers & 8710 & 8710 & & 11 & 8 & 6 \\
\hline \multirow[t]{2}{*}{ VIIa } & $\begin{array}{l}\text { Derrick, Rotary Drill, And Service Unit } \\
\text { Operators, And Roustabouts, Oil, Gas, And }\end{array}$ & & & & & & \\
\hline & Mining & 6800 & 6800 & & 0 & 4 & 0 \\
\hline VIIa & Dishwashers & 4140 & 4140 & & 12 & 26 & 11 \\
\hline \multirow[t]{2}{*}{ VIIa } & Dredge, Excavating, And Loading Machine & & & & & & \\
\hline & Operators & 9520 & 9520 & & 1 & 6 & 4 \\
\hline VIIa & Driver/Sales Workers And Truck Drivers & 9130 & 9130 & & 191 & 214 & 156 \\
\hline VIIa & Earth Drillers, Except Oil And Gas & 6820 & 6820 & & 3 & 2 & 1 \\
\hline \multirow[t]{2}{*}{ VIIa } & Electrical, Electronics, And Electromechanical & & & & & & \\
\hline & Assemblers & 7720 & 7720 & & 49 & 20 & 15 \\
\hline VIIa & Engine And Other Machine Assemblers & 7730 & 7730 & & 6 & 1 & 6 \\
\hline VIIa & $\begin{array}{l}\text { Extruding And Drawing Machine Setters, } \\
\text { Operators, And Tenders, Metal And Plastic }\end{array}$ & 7920 & 7920 & & 1 & 1 & 3 \\
\hline \multirow[t]{2}{*}{ VIIa } & $\begin{array}{l}\text { Extruding, Forming, Pressing, And } \\
\text { Compacting Machine Setters, Operators, And }\end{array}$ & & & & & & \\
\hline & Tenders & 8720 & 8720 & & 8 & 7 & 3 \\
\hline VIIa & Fence Erectors & 6710 & 6710 & & 1 & 1 & 0 \\
\hline \multirow[t]{2}{*}{ VIIa } & Food And Tobacco Roasting, Baking, And & & & & & & \\
\hline & Drying Machine Operators And Tenders & 7830 & 7830 & & 1 & 2 & 4 \\
\hline VIIa & Food Batchmakers & 7840 & 7840 & & 4 & 9 & 6 \\
\hline \multirow[t]{2}{*}{ VIIa } & Food Cooking Machine Operators And & & & & & & \\
\hline & Tenders & 7850 & 7850 & & 4 & 0 & 1 \\
\hline VIIa & Food Preparation Workers & 4030 & 4030 & & 33 & 31 & 44 \\
\hline VIIa & Food Processing Workers, All Other & 7855 & 7855 & & 5 & 11 & 9 \\
\hline \multirow[t]{2}{*}{ VIIa } & Forging Machine Setters, Operators, And & & & & & & \\
\hline & Tenders, Metal And Plastic & 7930 & 7930 & & 2 & 0 & 2 \\
\hline \multirow[t]{2}{*}{ VIIa } & Furnace, Kiln, Oven, Drier, And Kettle & & & & & & \\
\hline & Operators And Tenders & 8730 & 8730 & & 2 & 1 & 0 \\
\hline VIIa & Furniture Finishers & 8510 & 8510 & & 3 & 6 & 1 \\
\hline VIIa & Glaziers & 6360 & 6360 & & 0 & 5 & 5 \\
\hline VIIa & Grounds Maintenance Workers & 4250 & 4250 & & 43 & 42 & 52 \\
\hline VIIa & Helpers, Construction Trades & 6600 & 6600 & & 7 & 5 & 4 \\
\hline \multirow[t]{2}{*}{ VIIa } & Helpers-Installation, Maintenance, And Repair & & & & & & \\
\hline & Workers & 7610 & 7610 & & 2 & 1 & 5 \\
\hline VIIa & Helpers-Production Workers & 8950 & 8950 & & 4 & 1 & 6 \\
\hline VIIa & Highway Maintenance Workers & 6730 & 6730 & & 5 & 5 & 6 \\
\hline VIIa & Industrial Truck And Tractor Operators & 9600 & 9600 & & 36 & 41 & 29 \\
\hline \multirow[t]{2}{*}{ VIIa } & Inspectors, Testers, Sorters, Samplers, And & & & & & & \\
\hline & Weighers & 8740 & 8740 & & 88 & 77 & 40 \\
\hline VIIa & Janitors And Building Cleaners & 4220 & 4220 & & 159 & 175 & 159 \\
\hline \multirow[t]{2}{*}{ VIIa } & Laborers And Freight, Stock, And Material & & & & & & \\
\hline & Movers, Hand & 9620 & 9620 & & 106 & 101 & 94 \\
\hline VIIa & Laundry And Dry-Cleaning Workers & 8300 & 8300 & & 28 & 14 & 11 \\
\hline VIIa & Logging Workers & 6130 & 6130 & & 6 & 12 & 7 \\
\hline
\end{tabular}




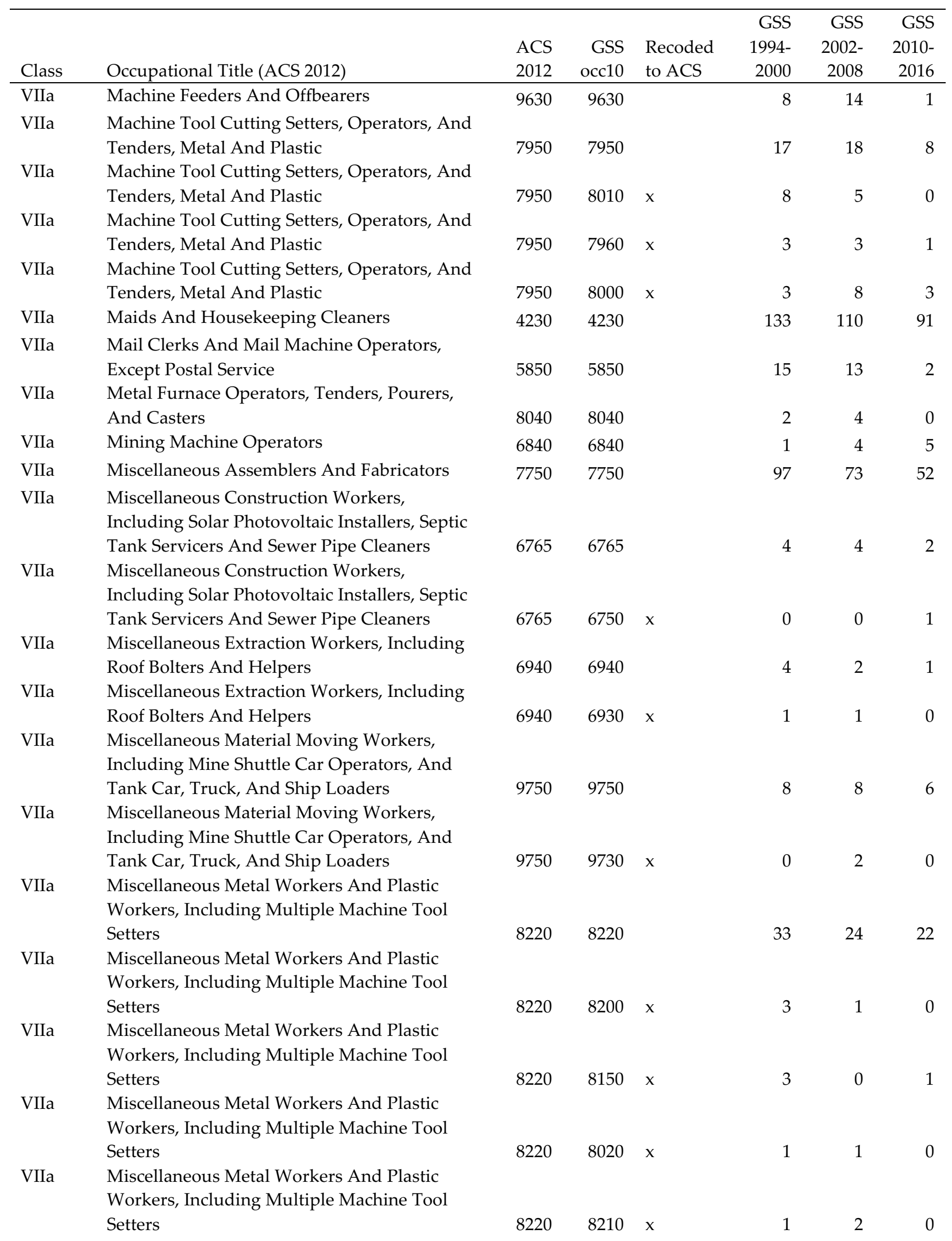




\begin{tabular}{|c|c|c|c|c|c|c|c|}
\hline Class & Occupational Title (ACS 2012) & $\begin{array}{l}\text { ACS } \\
2012\end{array}$ & $\begin{array}{r}\text { GSS } \\
\text { occ10 }\end{array}$ & $\begin{array}{l}\text { Recoded } \\
\text { to ACS }\end{array}$ & $\begin{array}{r}\text { GSS } \\
1994- \\
2000 \\
\end{array}$ & $\begin{array}{r}\text { GSS } \\
2002- \\
2008 \\
\end{array}$ & $\begin{array}{r}\text { GSS } \\
2010- \\
2016\end{array}$ \\
\hline \multirow[t]{2}{*}{ VIIa } & $\begin{array}{l}\text { Miscellaneous Metal Workers And Plastic } \\
\text { Workers, Including Multiple Machine Tool }\end{array}$ & & & & & & \\
\hline & Setters & 8220 & 8120 & $x$ & 0 & 2 & 0 \\
\hline \multirow[t]{2}{*}{ VIIa } & Miscellaneous Production Workers, Including & & & & & & \\
\hline & Semiconductor Processors & 8965 & 8965 & & 123 & 108 & 96 \\
\hline \multirow[t]{2}{*}{ VIIa } & Miscellaneous Production Workers, Including & & & & & & \\
\hline & Semiconductor Processors & 8965 & 8900 & $\mathrm{x}$ & 1 & 0 & 0 \\
\hline \multirow[t]{2}{*}{ VIIa } & Miscellaneous Production Workers, Including & & & & & & \\
\hline & Semiconductor Processors & 8965 & 8840 & $\mathrm{x}$ & 1 & 0 & 0 \\
\hline \multirow[t]{2}{*}{ VIIa } & Miscellaneous Production Workers, Including & & & & & & \\
\hline & Semiconductor Processors & 8965 & 8860 & $\mathrm{x}$ & 0 & 1 & 1 \\
\hline \multirow[t]{2}{*}{ VIIa } & Miscellaneous Textile, Apparel, And & & & & & & \\
\hline & Furnishings Workers, Except Upholsterers & 8460 & 8460 & & 8 & 7 & 2 \\
\hline \multirow[t]{2}{*}{ VIIa } & Miscellaneous Textile, Apparel, And & & & & & & \\
\hline & Furnishings Workers, Except Upholsterers & 8460 & 8440 & $\mathrm{x}$ & 2 & 0 & 0 \\
\hline \multirow[t]{2}{*}{ VIIa } & Miscellaneous Vehicle And Mobile Equipment & & & & & & \\
\hline & Mechanics, Installers, And Repairers & 7260 & 7260 & & 1 & 5 & 7 \\
\hline VIIa & Motor Vehicle Operators, All Other & 9150 & 9150 & & 3 & 3 & 0 \\
\hline \multirow[t]{2}{*}{ VIIa } & Packaging And Filling Machine Operators & & & & & & \\
\hline & And Tenders & 8800 & 8800 & & 27 & 16 & 19 \\
\hline VIIa & Packers And Packagers, Hand & 9640 & 9640 & & 35 & 34 & 28 \\
\hline VIIa & Painting Workers & 8810 & 8810 & & 9 & 11 & 9 \\
\hline \multirow[t]{2}{*}{ VIIa } & Paper Goods Machine Setters, Operators, And & & & & & & \\
\hline & Tenders & 8930 & 8930 & & 4 & 7 & 2 \\
\hline \multirow[t]{2}{*}{ VIIa } & Paving, Surfacing, And Tamping Equipment & & & & & & \\
\hline & Operators & 6300 & 6300 & & 0 & 1 & 0 \\
\hline \multirow[t]{2}{*}{ VIIa } & Photographic Process Workers And Processing & & & & & & \\
\hline & Machine Operators & 8830 & 8830 & & 10 & 9 & 3 \\
\hline VIIa & Plasterers And Stucco Masons & 6460 & 6460 & & 3 & 3 & 0 \\
\hline VIIa & Postal Service Mail Carriers & 5550 & 5550 & & 30 & 21 & 23 \\
\hline \multirow[t]{2}{*}{ VIIa } & Postal Service Mail Sorters, Processors, And & & & & & & \\
\hline & Processing Machine Operators & 5560 & 5560 & & 16 & 13 & 3 \\
\hline \multirow[t]{2}{*}{ VIIa } & Pressers, Textile, Garment, And Related & & & & & & \\
\hline & Materials & 8310 & 8310 & & 14 & 9 & 8 \\
\hline VIIa & Print Binding And Finishing Workers & 8256 & 8256 & & 9 & 4 & 3 \\
\hline VIIa & Pumping Station Operators & 9650 & 9650 & & 2 & 1 & 4 \\
\hline \multirow[t]{2}{*}{ VIIa } & Rail-Track Laying And Maintenance & & & & & & \\
\hline & Equipment Operators & 6740 & 6740 & & 1 & 3 & 0 \\
\hline VIIa & Refuse And Recyclable Material Collectors & 9720 & 9720 & & 2 & 8 & 10 \\
\hline \multirow[t]{2}{*}{ VIIa } & Rolling Machine Setters, Operators, And & & & & & & \\
\hline & Tenders, Metal And Plastic & 7940 & 7940 & & 3 & 0 & 1 \\
\hline VIIa & Roofers & 6515 & 6515 & & 11 & 17 & 10 \\
\hline \multirow[t]{2}{*}{ VIIa } & Sawing Machine Setters, Operators, And & & & & & & \\
\hline & Tenders, Wood & 8530 & 8530 & & 11 & 12 & 3 \\
\hline VIIa & Sewing Machine Operators & 8320 & 8320 & & 64 & 60 & 28 \\
\hline VIIa & Shipping, Receiving, And Traffic Clerks & 5610 & 5610 & & 50 & 38 & 25 \\
\hline VIIa & Shoe And Leather Workers & 8330 & 8330 & & 2 & 2 & 0 \\
\hline VIIa & Shoe And Leather Workers & 8330 & 8340 & $x$ & 10 & 0 & 3 \\
\hline
\end{tabular}




\begin{tabular}{|c|c|c|c|c|c|c|c|}
\hline Class & Occupational Title (ACS 2012) & $\begin{array}{l}\text { ACS } \\
2012\end{array}$ & $\begin{array}{r}\text { GSS } \\
\text { occ10 }\end{array}$ & $\begin{array}{l}\text { Recoded } \\
\text { to ACS }\end{array}$ & $\begin{array}{r}\text { GSS } \\
1994- \\
2000\end{array}$ & $\begin{array}{r}\text { GSS } \\
2002- \\
2008\end{array}$ & $\begin{array}{r}\text { GSS } \\
2010- \\
2016\end{array}$ \\
\hline VIIa & Stock Clerks And Order Fillers & 5620 & 5620 & & 101 & 83 & 63 \\
\hline VIIa & Taxi Drivers And Chauffeurs & 9140 & 9140 & & 10 & 15 & 12 \\
\hline VIIa & $\begin{array}{l}\text { Textile Bleaching And Dyeing, And Cutting } \\
\text { Machine Setters, Operators, And Tenders }\end{array}$ & 8400 & 8400 & & 3 & 1 & 0 \\
\hline VIIa & Textile Bleaching And Dyeing, And Cutting & & & & & & \\
\hline VIIa & $\begin{array}{l}\text { Machine Setters, Operators, And Tenders } \\
\text { Textile Knitting And Weaving Machine }\end{array}$ & 8400 & 8360 & $\mathrm{x}$ & 0 & 0 & 2 \\
\hline VIIa & $\begin{array}{l}\text { Setters, Operators, And Tenders } \\
\text { Textile Winding, Twisting, And Drawing Out }\end{array}$ & 8410 & 8410 & & 8 & 3 & 1 \\
\hline & Machine Setters, Operators, And Tenders & 8420 & 8420 & & 12 & 12 & 2 \\
\hline VIIa & Tire Builders & 8940 & 8940 & & 2 & 2 & 1 \\
\hline VIIa & Upholsterers & 8450 & 8450 & & 7 & 6 & 0 \\
\hline VIIa & Welding, Soldering, And Brazing Workers & 8140 & 8140 & & 59 & 49 & 34 \\
\hline VIIa & $\begin{array}{l}\text { Woodworking Machine Setters, Operators, } \\
\text { And Tenders, Except Sawing }\end{array}$ & 8540 & 8540 & & 2 & 6 & 3 \\
\hline VIIb & Agricultural Inspectors & 6010 & 6010 & & 1 & 2 & 1 \\
\hline VIIb & Animal Trainers & 4340 & 4340 & & 0 & 4 & 1 \\
\hline VIIb & First-Line Supervisors Of Farming, Fishing, & (600- & 5 & & 2 & 6 & 4 \\
\hline VIIb & Fishing And Hunting Workers & 6100 & 6100 & & 1 & 2 & 1 \\
\hline VIIb & Graders And Sorters, Agricultural Products & 6040 & 6040 & & 5 & 6 & 4 \\
\hline $\mathrm{VIIb}$ & $\begin{array}{l}\text { Miscellaneous Agricultural Workers, } \\
\text { Including Animal Breeders }\end{array}$ & 6050 & 6050 & & 48 & 70 & 42 \\
\hline Mil & First-Line Enlisted Military Supervisors & 9810 & 9810 & & 16 & 15 & 35 \\
\hline Mil & Military Enlisted Tactical Operations And & & & & & & \\
\hline Mil & $\begin{array}{l}\text { Air/Weapons Specialists And Crew Members } \\
\text { Military Officer Special And Tactical }\end{array}$ & 9820 & 9820 & & 8 & 15 & 7 \\
\hline & Operations Leaders & 9800 & 9800 & & 7 & 10 & 38 \\
\hline Mil & Military, Rank Not Specified & 9830 & 9830 & & 13 & 23 & 41 \\
\hline
\end{tabular}


Table A3. GSS Occupational Distributions for the 12-Class Version of EGP

\begin{tabular}{|c|c|c|c|c|c|}
\hline Class & Occupational Title (ACS 2012) & $\begin{array}{l}\text { ACS } \\
2012 \\
\end{array}$ & $\begin{array}{r}\text { GSS } \\
\text { occ10 }\end{array}$ & $\begin{array}{l}\text { Recoded } \\
\text { to ACS }\end{array}$ & $\begin{array}{r}\text { GSS } \\
2004- \\
2016 \\
\end{array}$ \\
\hline I & Accountants And Auditors & 800 & 800 & & 188 \\
\hline I & Aerospace Engineers & 1320 & 1320 & & 26 \\
\hline I & Agricultural And Food Scientists & 1600 & 1600 & & 5 \\
\hline I & Architects, Except Naval & 1300 & 1300 & & 27 \\
\hline I & Architectural And Engineering Managers & 300 & 300 & & 14 \\
\hline I & Astronomers And Physicists & 1700 & 1700 & & 1 \\
\hline I & Atmospheric And Space Scientists & 1710 & 1710 & & 2 \\
\hline I & Biological Scientists & 1610 & 1610 & & 18 \\
\hline I & Budget Analysts & 820 & 820 & & 9 \\
\hline I & Chemical Engineers & 1350 & 1350 & & 8 \\
\hline I & Chemists And Materials Scientists & 1720 & 1720 & & 14 \\
\hline I & Chief Executives And Legislators & 10 & 10 & & 113 \\
\hline I & Chief Executives And Legislators & 10 & 30 & $x$ & 3 \\
\hline I & Chiropractors & 3000 & 3000 & & 10 \\
\hline I & Civil Engineers & 1360 & 1360 & & 42 \\
\hline I & $\begin{array}{l}\text { Computer And Information Research } \\
\text { Scientists }\end{array}$ & 1005 & 1005 & & 2 \\
\hline I & Computer And Information Systems & & & & \\
\hline & Managers & 110 & 110 & & 42 \\
\hline I & Computer Hardware Engineers & 1400 & 1400 & & 5 \\
\hline I & Conservation Scientists And Foresters & 1640 & 1640 & & 9 \\
\hline I & Dentists & 3010 & 3010 & & 12 \\
\hline I & Economists & 1800 & 1800 & & 4 \\
\hline I & Education Administrators & 230 & 230 & & 101 \\
\hline I & Electrical And Electronics Engineers & 1410 & 1410 & & 44 \\
\hline I & Environmental Engineers & 1420 & 1420 & & 11 \\
\hline I & Environmental Scientists And Geoscientists & 1740 & 1740 & & 16 \\
\hline I & Financial Analysts & 840 & 840 & & 16 \\
\hline I & $\begin{array}{l}\text { Industrial Engineers, Including Health And } \\
\text { Safety }\end{array}$ & 1430 & 1430 & & 36 \\
\hline I & Judicial Law Clerks & 2105 & 2105 & & 2 \\
\hline I & $\begin{array}{l}\text { Lawyers, And Judges, Magistrates, And Other } \\
\text { Judicial Workers }\end{array}$ & 2100 & 2100 & & 97 \\
\hline I & $\begin{array}{l}\text { Lawyers, And Judges, Magistrates, And Other } \\
\text { Judicial Workers }\end{array}$ & 2100 & 2110 & $x$ & 5 \\
\hline I & Management Analysts & 710 & 710 & & 84 \\
\hline I & Marine Engineers And Naval Architects & 1440 & 1440 & & 1 \\
\hline I & Materials Engineers & 1450 & 1450 & & 4 \\
\hline I & Mechanical Engineers & 1460 & 1460 & & 53 \\
\hline I & Medical And Health Services Managers & 350 & 350 & & 68 \\
\hline I & $\begin{array}{l}\text { Medical Scientists, And Life Scientists, All } \\
\text { Other }\end{array}$ & 1650 & 1650 & & 17 \\
\hline I & $\begin{array}{l}\text { Miscellaneous Engineers, Including Nuclear } \\
\text { Engineers }\end{array}$ & 1530 & 1530 & & 21 \\
\hline
\end{tabular}




\begin{tabular}{|c|c|c|c|c|c|}
\hline Class & Occupational Title (ACS 2012) & $\begin{array}{l}\text { ACS } \\
2012\end{array}$ & $\begin{array}{r}\text { GSS } \\
\text { occ10 }\end{array}$ & $\begin{array}{l}\text { Recoded } \\
\text { to ACS }\end{array}$ & $\begin{array}{r}\text { GSS } \\
2004- \\
2016\end{array}$ \\
\hline \multirow[t]{2}{*}{$\mathrm{I}$} & Miscellaneous Engineers, Including Nuclear & & & & \\
\hline & Engineers & 1530 & 1510 & $x$ & 3 \\
\hline \multirow[t]{2}{*}{ I } & Miscellaneous Mathematical Science & & & & \\
\hline & $\begin{array}{l}\text { Occupations, Including Mathematicians And } \\
\text { Statisticians }\end{array}$ & 1240 & 1230 & $\mathrm{x}$ & 3 \\
\hline \multirow[t]{3}{*}{ I } & Miscellaneous Mathematical Science & & & & \\
\hline & Occupations, Including Mathematicians And & & & & \\
\hline & Statisticians & 1240 & 1210 & $x$ & 2 \\
\hline I & Natural Sciences Managers & 360 & 360 & & 4 \\
\hline I & Operations Research Analysts & 1220 & 1220 & & 17 \\
\hline I & Optometrists & 3040 & 3040 & & 2 \\
\hline I & $\begin{array}{l}\text { Petroleum, Mining And Geological Engineers, } \\
\text { Including Mining Safety Engineers }\end{array}$ & 1520 & 1520 & & 3 \\
\hline I & $\begin{array}{l}\text { Petroleum, Mining And Geological Engineers, } \\
\text { Including Mining Safety Engineers }\end{array}$ & 1520 & 1500 & $\mathrm{x}$ & 1 \\
\hline I & Pharmacists & 3050 & 3050 & & 22 \\
\hline I & Physical Scientists, All Other & 1760 & 1760 & & 13 \\
\hline I & Physicians And Surgeons & 3060 & 3060 & & 67 \\
\hline I & Podiatrists & 3120 & 3120 & & 1 \\
\hline I & Postsecondary Teachers & 2200 & 2200 & & 197 \\
\hline I & Psychologists & 1820 & 1820 & & 26 \\
\hline \multirow[t]{2}{*}{ I } & Software Developers, Applications And & & & & \\
\hline & Systems Software & 1020 & 1020 & & 123 \\
\hline I & Urban And Regional Planners & 1840 & 1840 & & 7 \\
\hline $\mathrm{I}$ & Veterinarians & 3250 & 3250 & & 7 \\
\hline II & Advertising And Promotions Managers & 40 & 40 & & 6 \\
\hline II & $\begin{array}{l}\text { Agents And Business Managers Of Artists, } \\
\text { Performers, And Athletes }\end{array}$ & 500 & 500 & & 8 \\
\hline II & Aircraft Pilots And Flight Engineers & 9030 & 9030 & & 15 \\
\hline \multirow[t]{2}{*}{ II } & Archivists, Curators, And Museum & & & & \\
\hline & Technicians & 2400 & 2400 & & 13 \\
\hline II & Business Operations Specialists, All Other & 740 & 740 & & 47 \\
\hline II & Clergy & 2040 & 2040 & & 45 \\
\hline II & Compensation And Benefits Managers & 135 & 135 & & 8 \\
\hline \multirow[t]{2}{*}{ II } & Compensation, Benefits, And Job Analysis & & & & \\
\hline & Specialists & 640 & 640 & & 10 \\
\hline II & Compliance Officers & 565 & 565 & & 22 \\
\hline II & Computer Network Architects & 1106 & 1106 & & 13 \\
\hline II & Computer Occupations, All Other & 1107 & 1107 & & 20 \\
\hline II & Computer Programmers & 1010 & 1010 & & 54 \\
\hline II & Computer Systems Analysts & 1006 & 1006 & & 62 \\
\hline II & Counselors & 2000 & 2000 & & 90 \\
\hline II & Database Administrators & 1060 & 1060 & & 12 \\
\hline II & Dietitians And Nutritionists & 3030 & 3030 & & 13 \\
\hline II & Directors, Religious Activities And Education & 2050 & 2050 & & 6 \\
\hline II & Editors & 2830 & 2830 & & 26 \\
\hline
\end{tabular}




\begin{tabular}{|c|c|c|c|c|c|}
\hline Class & Occupational Title (ACS 2012) & $\begin{array}{l}\text { ACS } \\
2012 \\
\end{array}$ & $\begin{array}{r}\text { GSS } \\
\text { occ10 }\end{array}$ & $\begin{array}{l}\text { Recoded } \\
\text { to ACS }\end{array}$ & $\begin{array}{r}\text { GSS } \\
2004- \\
2016 \\
\end{array}$ \\
\hline II & Elementary And Middle School Teachers & 2310 & 2310 & & 397 \\
\hline II & Emergency Management Directors & 425 & 425 & & 1 \\
\hline II & Financial Examiners & 900 & 900 & & 2 \\
\hline II & Financial Managers & 120 & 120 & & 125 \\
\hline II & Financial Specialists, All Other & 950 & 950 & & 6 \\
\hline II & Fundraisers & 726 & 726 & & 5 \\
\hline II & General And Operations Managers & 20 & 20 & & 121 \\
\hline II & $\begin{array}{l}\text { Health Diagnosing And Treating Practitioners, } \\
\text { All Other }\end{array}$ & 3260 & 3260 & & 5 \\
\hline II & Human Resources Managers & 136 & 136 & & 26 \\
\hline II & Industrial Production Managers & 140 & 140 & & 40 \\
\hline II & Information Security Analysts & 1007 & 1007 & & 7 \\
\hline II & Insurance Underwriters & 860 & 860 & & 11 \\
\hline II & Librarians & 2430 & 2430 & & 40 \\
\hline II & Logisticians & 700 & 700 & & 13 \\
\hline II & Market Research Analysts And Marketing & & & & \\
\hline & Specialists & 735 & 735 & & 21 \\
\hline II & Marketing And Sales Managers & 50 & 50 & & 111 \\
\hline II & $\begin{array}{l}\text { Miscellaneous Community And Social Service } \\
\text { Specialists, Including Health Educators And }\end{array}$ & & & & \\
\hline II & $\begin{array}{l}\text { Community Health Workers } \\
\text { Miscellaneous Managers, Including Funeral } \\
\text { Service Managers And Postmasters And Mail }\end{array}$ & 2025 & 2025 & & 16 \\
\hline II & $\begin{array}{l}\text { Superintendents } \\
\text { Miscellaneous Managers, Including Funeral } \\
\text { Service Managers And Postmasters And Mail }\end{array}$ & 430 & 430 & & 304 \\
\hline II & $\begin{array}{l}\text { Superintendents } \\
\text { Miscellaneous Managers, Including Funeral } \\
\text { Service Managers And Postmasters And Mail }\end{array}$ & 430 & 325 & $\mathrm{x}$ & 4 \\
\hline II & $\begin{array}{l}\text { Superintendents } \\
\text { Miscellaneous Social Scientists, Including }\end{array}$ & 430 & 400 & $x$ & 8 \\
\hline & Survey Researchers And Sociologists & 1860 & 1860 & & 12 \\
\hline II & Miscellaneous Social Scientists, Including & & & & \\
\hline & Survey Researchers And Sociologists & 1860 & 1815 & $\mathrm{x}$ & 1 \\
\hline II & $\begin{array}{l}\text { Miscellaneous Social Scientists, Including } \\
\text { Survey Researchers And Sociologists }\end{array}$ & 1860 & 1830 & $x$ & 1 \\
\hline II & $\begin{array}{l}\text { Network And Computer Systems } \\
\text { Administrators }\end{array}$ & 1105 & 1105 & & 34 \\
\hline II & News Analysts, Reporters And & & & & \\
\hline & Correspondents & 2810 & 2810 & & 11 \\
\hline II & Nurse Anesthetists & 3256 & 3256 & & 5 \\
\hline II & Nurse Practitioners, And Nurse Midwives & 3258 & 3258 & & 9 \\
\hline II & Occupational Therapists & 3150 & 3150 & & 8 \\
\hline II & Other Education, Training, And Library & & & & \\
\hline & Workers & 2550 & 2550 & & 12 \\
\hline II & $\begin{array}{l}\text { Other Healthcare Practitioners And Technical } \\
\text { Occupations }\end{array}$ & 3540 & 3540 & & 14 \\
\hline
\end{tabular}




\begin{tabular}{|c|c|c|c|c|c|}
\hline Class & Occupational Title (ACS 2012) & $\begin{array}{l}\text { ACS } \\
2012\end{array}$ & $\begin{array}{r}\text { GSS } \\
\text { occ10 }\end{array}$ & $\begin{array}{l}\text { Recoded } \\
\text { to ACS }\end{array}$ & $\begin{array}{r}\text { GSS } \\
2004- \\
2016 \\
\end{array}$ \\
\hline \multirow[t]{2}{*}{ II } & Other Therapists, Including Exercise & & & & \\
\hline & Physiologists & 3245 & 3245 & & 17 \\
\hline II & Personal Financial Advisors & 850 & 850 & & 38 \\
\hline II & Physical Therapists & 3160 & 3160 & & 16 \\
\hline II & Physician Assistants & 3110 & 3110 & & 9 \\
\hline II & Producers And Directors & 2710 & 2710 & & 29 \\
\hline II & Public Relations And Fundraising Managers & 60 & 60 & & 11 \\
\hline II & Public Relations Specialists & 2825 & 2825 & & 18 \\
\hline II & $\begin{array}{l}\text { Purchasing Agents, Except Wholesale, Retail, } \\
\text { And Farm Products }\end{array}$ & 530 & 530 & & 34 \\
\hline II & Purchasing Managers & 150 & 150 & & 20 \\
\hline II & Recreational Therapists & 3210 & 3210 & & 4 \\
\hline II & Registered Nurses & 3255 & 3255 & & 266 \\
\hline II & Sales Engineers & 4930 & 4930 & & 3 \\
\hline II & Secondary School Teachers & 2320 & 2320 & & 211 \\
\hline II & $\begin{array}{l}\text { Securities, Commodities, And Financial } \\
\text { Services Sales Agents }\end{array}$ & 4820 & 4820 & & 28 \\
\hline II & Social And Community Service Managers & 420 & 420 & & 45 \\
\hline II & Social Workers & 2010 & 2010 & & 125 \\
\hline II & Special Education Teachers & 2330 & 2330 & & 56 \\
\hline II & Speech-Language Pathologists & 3230 & 3230 & & 19 \\
\hline \multirow[t]{2}{*}{ II } & Surveyors, Cartographers, And & & & & \\
\hline & Photogrammetrists & 1310 & 1310 & & 6 \\
\hline II & Technical Writers & 2840 & 2840 & & 5 \\
\hline II & Training And Development Managers & 137 & 137 & & 7 \\
\hline II & Training And Development Specialists & 650 & 650 & & 17 \\
\hline II & Writers And Authors & 2850 & 2850 & & 27 \\
\hline IIIa & Administrative Services Managers & 100 & 100 & & 11 \\
\hline IIIa & Advertising Sales Agents & 4800 & 4800 & & 22 \\
\hline IIIIa & $\begin{array}{l}\text { Air Traffic Controllers And Airfield } \\
\text { Operations Specialists }\end{array}$ & 9040 & 9040 & & 9 \\
\hline IIIa & Appraisers And Assessors Of Real Estate & 810 & 810 & & 6 \\
\hline IIIa & Bill And Account Collectors & 5100 & 5100 & & 21 \\
\hline IIIa & Billing And Posting Clerks & 5110 & 5110 & & 60 \\
\hline IIIa & $\begin{array}{l}\text { Bookkeeping, Accounting, And Auditing } \\
\text { Clerks }\end{array}$ & 5120 & 5120 & & 163 \\
\hline IIIa & Brokerage Clerks & 5200 & 5200 & & 2 \\
\hline IIIa & Cargo And Freight Agents & 5500 & 5500 & & 2 \\
\hline IIIIa & $\begin{array}{l}\text { Claims Adjusters, Appraisers, Examiners, And } \\
\text { Investigators }\end{array}$ & 540 & 540 & & 36 \\
\hline IIIa & $\begin{array}{l}\text { Communications Equipment Operators, All } \\
\text { Other }\end{array}$ & 5030 & 5030 & & 1 \\
\hline IIIa & Computer Operators & 5800 & 5800 & & 19 \\
\hline IIIa & Computer Support Specialists & 1050 & 1050 & & 67 \\
\hline IIIa & Correspondence Clerks And Order Clerks & 5350 & 5350 & & 10 \\
\hline
\end{tabular}




\begin{tabular}{|c|c|c|c|c|c|}
\hline Class & Occupational Title (ACS 2012) & $\begin{array}{l}\text { ACS } \\
2012\end{array}$ & $\begin{array}{r}\text { GSS } \\
\text { occ10 }\end{array}$ & $\begin{array}{l}\text { Recoded } \\
\text { to ACS }\end{array}$ & $\begin{array}{r}\text { GSS } \\
2004- \\
2016\end{array}$ \\
\hline IIIa & Court, Municipal, And License Clerks & 5220 & 5220 & & 12 \\
\hline IIIa & Credit Analysts & 830 & 830 & & 6 \\
\hline IIIa & Credit Authorizers, Checkers, And Clerks & 5230 & 5230 & & 5 \\
\hline IIIa & Credit Counselors And Loan Officers & 910 & 910 & & 32 \\
\hline IIIa & Customer Service Representatives & 5240 & 5240 & & 254 \\
\hline IIIa & Data Entry Keyers & 5810 & 5810 & & 47 \\
\hline IIIa & Dispatchers & 5520 & 5520 & & 36 \\
\hline \multirow[t]{2}{*}{ IIIa } & Eligibility Interviewers, Government & & & & \\
\hline & Programs & 5250 & 5250 & & 7 \\
\hline IIIa & File Clerks & 5260 & 5260 & & 17 \\
\hline IIIa & Financial Clerks, All Other & 5165 & 5165 & & 5 \\
\hline \multirow[t]{2}{*}{ IIIa } & First-Line Supervisors Of Office And & & & & \\
\hline & Administrative Support Workers & 5000 & 5000 & & 219 \\
\hline IIIa & Flight Attendants & 9050 & 9050 & & 11 \\
\hline IIIa & Hotel, Motel, And Resort Desk Clerks & 5300 & 5300 & & 19 \\
\hline IIIa & $\begin{array}{l}\text { Human Resources Assistants, Except Payroll } \\
\text { And Timekeeping }\end{array}$ & 5360 & 5360 & & 20 \\
\hline IIIa & Human Resources Workers & 630 & 630 & & 62 \\
\hline IIIa & Information And Record Clerks, All Other & 5420 & 5420 & & 13 \\
\hline IIIa & $\begin{array}{l}\text { Insurance Claims And Policy Processing } \\
\text { Clerks }\end{array}$ & 5840 & 5840 & & 32 \\
\hline IIIa & Insurance Sales Agents & 4810 & 4810 & & 57 \\
\hline IIIa & Interviewers, Except Eligibility And Loan & 5310 & 5310 & & 26 \\
\hline IIIa & Library Assistants, Clerical & 5320 & 5320 & & 13 \\
\hline IIIa & Library Technicians & 2440 & 2440 & & 6 \\
\hline IIIa & $\begin{array}{l}\text { Licensed Practical And Licensed Vocational } \\
\text { Nurses }\end{array}$ & 3500 & 3500 & & 59 \\
\hline IIIa & Loan Interviewers And Clerks & 5330 & 5330 & & 19 \\
\hline IIIa & Lodging Managers & 340 & 340 & & 15 \\
\hline IIIa & $\begin{array}{l}\text { Medical Records And Health Information } \\
\text { Technicians }\end{array}$ & 3510 & 3510 & & 14 \\
\hline IIIa & Meeting, Convention, And Event Planners & 725 & 725 & & 5 \\
\hline IIIa & Meter Readers, Utilities & 5530 & 5530 & & 2 \\
\hline IIIa & Miscellaneous Legal Support Workers & 2160 & 2160 & & 24 \\
\hline IIIIa & $\begin{array}{l}\text { Miscellaneous Office And Administrative } \\
\text { Support Workers, Including Desktop }\end{array}$ & & & & \\
\hline \multirow{3}{*}{ IIIa } & Publishers & 5940 & 5940 & & 76 \\
\hline & $\begin{array}{l}\text { Miscellaneous Office And Administrative } \\
\text { Support Workers, Including Desktop }\end{array}$ & & & & \\
\hline & Publishers & 5940 & 5830 & $\mathrm{x}$ & 1 \\
\hline IIIa & Office Clerks, General & 5860 & 5860 & & 150 \\
\hline IIIa & Office Machine Operators, Except Computer & 5900 & 5900 & & 4 \\
\hline IIIa & Other Teachers And Instructors & 2340 & 2340 & & 61 \\
\hline IIIa & Paralegals And Legal Assistants & 2145 & 2145 & & 40 \\
\hline IIIa & Payroll And Timekeeping Clerks & 5140 & 5140 & & 28 \\
\hline IIIa & Postal Service Clerks & 5540 & 5540 & & 21 \\
\hline
\end{tabular}




\begin{tabular}{|c|c|c|c|c|c|}
\hline Class & Occupational Title (ACS 2012) & $\begin{array}{l}\text { ACS } \\
2012\end{array}$ & $\begin{array}{r}\text { GSS } \\
\text { occ10 }\end{array}$ & $\begin{array}{l}\text { Recoded } \\
\text { to ACS }\end{array}$ & $\begin{array}{r}\text { GSS } \\
2004- \\
2016 \\
\end{array}$ \\
\hline IIIa & Procurement Clerks & 5150 & 5150 & & 2 \\
\hline IIIa & Production, Planning, And Expediting Clerks & 5600 & 5600 & & 43 \\
\hline IIIa & Proofreaders And Copy Markers & 5910 & 5910 & & 1 \\
\hline IIIa & Radiation Therapists & 3200 & 3200 & & 2 \\
\hline IIIa & Real Estate Brokers And Sales Agents & 4920 & 4920 & & 41 \\
\hline IIIa & $\begin{array}{l}\text { Reservation And Transportation Ticket Agents } \\
\text { And Travel Clerks }\end{array}$ & 5410 & 5410 & & 16 \\
\hline IIIa & Respiratory Therapists & 3220 & 3220 & & 8 \\
\hline IIIa & Sales And Related Workers, All Other & 4965 & 4965 & & 60 \\
\hline IIIa & Sales Representatives, Services, All Other & 4840 & 4840 & & 28 \\
\hline IIIa & $\begin{array}{l}\text { Sales Representatives, Wholesale And } \\
\text { Manufacturing }\end{array}$ & 4850 & 4850 & & 132 \\
\hline IIIa & Secretaries And Administrative Assistants & 5700 & 5700 & & 519 \\
\hline IIIa & Social And Human Service Assistants & 2016 & 2016 & & 18 \\
\hline IIIa & Statistical Assistants & 5920 & 5920 & & 6 \\
\hline IIIa & $\begin{array}{l}\text { Switchboard Operators, Including Answering } \\
\text { Service }\end{array}$ & 5010 & 5010 & & 15 \\
\hline IIIa & $\begin{array}{l}\text { Tax Examiners And Collectors, And Revenue } \\
\text { Agents }\end{array}$ & 930 & 930 & & 10 \\
\hline IIIa & Tax Preparers & 940 & 940 & & 13 \\
\hline IIIa & Teacher Assistants & 2540 & 2540 & & 120 \\
\hline IIIa & Telephone Operators & 5020 & 5020 & & 15 \\
\hline IIIa & Tellers & 5160 & 5160 & & 57 \\
\hline IIIa & Tour And Travel Guides & 4540 & 4540 & & 3 \\
\hline IIIa & Travel Agents & 4830 & 4830 & & 7 \\
\hline IIIa & Web Developers & 1030 & 1030 & & 17 \\
\hline IIIa & $\begin{array}{l}\text { Weighers, Measurers, Checkers, And } \\
\text { Samplers, Recordkeeping }\end{array}$ & 5630 & 5630 & & 17 \\
\hline IIIa & Word Processors And Typists & 5820 & 5820 & & 29 \\
\hline $\mathrm{IIIb}$ & Animal Control Workers & 3900 & 3900 & & 1 \\
\hline IIIb & $\begin{array}{l}\text { Automotive And Watercraft Service } \\
\text { Attendants }\end{array}$ & 9360 & 9360 & & 8 \\
\hline $\mathrm{IIIb}$ & Baggage Porters, Bellhops, And Concierges & 4530 & 4530 & & 20 \\
\hline $\mathrm{IIIb}$ & Barbers & 4500 & 4500 & & 6 \\
\hline $\mathrm{IIIb}$ & Bartenders & 4040 & 4040 & & 55 \\
\hline $\mathrm{IIIb}$ & Bus Drivers & 9120 & 9120 & & 66 \\
\hline $\mathrm{IIIb}$ & Cashiers & 4720 & 4720 & & 288 \\
\hline $\mathrm{IIIb}$ & Childcare Workers & 4600 & 4600 & & 126 \\
\hline IIIb & $\begin{array}{l}\text { Coin, Vending, And Amusement Machine } \\
\text { Servicers And Repairers }\end{array}$ & 7510 & 7510 & & 6 \\
\hline $\mathrm{IIIb}$ & $\begin{array}{l}\text { Combined Food Preparation And Serving } \\
\text { Workers, Including Fast Food }\end{array}$ & 4050 & 4050 & & 83 \\
\hline $\mathrm{IIIb}$ & Counter And Rental Clerks & 4740 & 4740 & & 17 \\
\hline IIIb & Counter Attendants, Cafeteria, Food & & & & \\
\hline & Concession, And Coffee Shop & 4060 & 4060 & & 22 \\
\hline $\mathrm{IIIb}$ & Couriers And Messengers & 5510 & 5510 & & 32 \\
\hline
\end{tabular}




\begin{tabular}{|c|c|c|c|c|c|}
\hline Class & Occupational Title (ACS 2012) & $\begin{array}{l}\text { ACS } \\
2012 \\
\end{array}$ & $\begin{array}{r}\text { GSS } \\
\text { occ10 }\end{array}$ & $\begin{array}{l}\text { Recoded } \\
\text { to ACS }\end{array}$ & $\begin{array}{r}\text { GSS } \\
2004- \\
2016 \\
\end{array}$ \\
\hline IIIb & Dental Assistants & 3640 & 3640 & & 38 \\
\hline \multirow[t]{2}{*}{$\mathrm{IIIb}$} & Door-To-Door Sales Workers, News And & & & & \\
\hline & Street Vendors, And Related Workers & 4950 & 4950 & & 8 \\
\hline $\mathrm{IIIb}$ & Embalmers And Funeral Attendants & 4460 & 4460 & & 2 \\
\hline $\mathrm{IIIb}$ & First-Line Supervisors Of Gaming Workers & 4300 & 4300 & & 2 \\
\hline $\mathrm{IIIb}$ & $\begin{array}{l}\text { First-Line Supervisors Of Housekeeping And } \\
\text { Janitorial Workers }\end{array}$ & 4200 & 4200 & & 27 \\
\hline \multirow[t]{2}{*}{ IIIb } & First-Line Supervisors Of Personal Service & & & & \\
\hline & Workers & 4320 & 4320 & & 8 \\
\hline IIIb & First-Line Supervisors Of Retail Sales Workers & 4700 & 4700 & & 247 \\
\hline \multirow[t]{2}{*}{$\mathrm{IIIb}$} & First-Line Supervisors Of Food Preparation & & & & \\
\hline & And Serving Workers & 4010 & 4010 & & 86 \\
\hline IIIb & Food Servers, Nonrestaurant & 4120 & 4120 & & 17 \\
\hline $\mathrm{IIIb}$ & Food Service Managers & 310 & 310 & & 47 \\
\hline $\mathrm{IIIb}$ & Gaming Services Workers & 4400 & 4400 & & 5 \\
\hline $\mathrm{IIIb}$ & Hairdressers, Hairstylists, And Cosmetologists & 4510 & 4510 & & 52 \\
\hline IIIb & $\begin{array}{l}\text { Healthcare Support Workers, All Other, } \\
\text { Including Medical Equipment Preparers }\end{array}$ & 3655 & 3655 & & 31 \\
\hline IIIb & Hosts And Hostesses, Restaurant, Lounge, & & & & \\
\hline & And Coffee Shop & 4150 & 4150 & & 14 \\
\hline \multirow[t]{2}{*}{ IIIb } & Lifeguards And Other Recreational, And All & & & & \\
\hline & Other Protective Service Workers & 3955 & 3955 & & 7 \\
\hline IIIb & Massage Therapists & 3630 & 3630 & & 13 \\
\hline $\mathrm{IIIb}$ & Medical Assistants & 3645 & 3645 & & 43 \\
\hline $\mathrm{IIIb}$ & Medical Transcriptionists & 3646 & 3646 & & 8 \\
\hline \multirow[t]{2}{*}{$\mathrm{IIIb}$} & Miscellaneous Entertainment Attendants And & & & & \\
\hline & Related Workers & 4430 & 4430 & & 25 \\
\hline $\mathrm{IIIb}$ & $\begin{array}{l}\text { Miscellaneous Food Preparation And Serving } \\
\text { Related Workers, Including Dining Room And }\end{array}$ & & & & \\
\hline & Cafeteria Attendants And Bartender Helpers & 4130 & 4130 & & 42 \\
\hline \multirow[t]{3}{*}{$\mathrm{IIIb}$} & Miscellaneous Food Preparation And Serving & & & & \\
\hline & Related Workers, Including Dining Room And & & & & \\
\hline & Cafeteria Attendants And Bartender Helpers & 4130 & 4160 & $\mathrm{x}$ & 3 \\
\hline $\mathrm{IIIb}$ & Miscellaneous Personal Appearance Workers & 4520 & 4520 & & 10 \\
\hline $\mathrm{IIIb}$ & Models, Demonstrators, And Product & & & & \\
\hline & Promoters & 4900 & 4900 & & 8 \\
\hline \multirow[t]{2}{*}{ IIIb } & Morticians, Undertakers, And Funeral & & & & \\
\hline & Directors & 4465 & 4465 & & 5 \\
\hline $\mathrm{IIIb}$ & Motion Picture Projectionists & 4410 & 4410 & & 2 \\
\hline IIIb & Nonfarm Animal Caretakers & 4350 & 4350 & & 9 \\
\hline $\mathrm{IIIb}$ & Nursing, Psychiatric, And Home Health Aides & 3600 & 3600 & & 431 \\
\hline $\mathrm{IIIb}$ & Parking Lot Attendants & 9350 & 9350 & & 5 \\
\hline $\mathrm{IIIb}$ & Parts Salespersons & 4750 & 4750 & & 23 \\
\hline IIIb & Personal Care Aides & 4610 & 4610 & & 78 \\
\hline $\mathrm{IIIb}$ & Personal Care And Service Workers, All Other & 4650 & 4650 & & 9 \\
\hline IIIb & Pest Control Workers & 4240 & 4240 & & 4 \\
\hline $\mathrm{IIIb}$ & Pharmacy Aides & 3647 & 3647 & & 10 \\
\hline
\end{tabular}




\begin{tabular}{|c|c|c|c|c|c|}
\hline Class & Occupational Title (ACS 2012) & $\begin{array}{l}\text { ACS } \\
2012\end{array}$ & $\begin{array}{r}\text { GSS } \\
\text { occ10 }\end{array}$ & $\begin{array}{l}\text { Recoded } \\
\text { to ACS }\end{array}$ & $\begin{array}{r}\text { GSS } \\
2004- \\
2016\end{array}$ \\
\hline IIIlb & Phlebotomists & 3649 & 3649 & & 18 \\
\hline $\mathrm{IIIb}$ & Physical Therapist Assistants And Aides & 3620 & 3620 & & 8 \\
\hline $\mathrm{IIIb}$ & Preschool And Kindergarten Teachers & 2300 & 2300 & & 94 \\
\hline $\mathrm{IIIb}$ & Receptionists And Information Clerks & 5400 & 5400 & & 102 \\
\hline IIIlb & Recreation And Fitness Workers & 4620 & 4620 & & 40 \\
\hline $\mathrm{IIIb}$ & Religious Workers, All Other & 2060 & 2060 & & 10 \\
\hline $\mathrm{IIIb}$ & Residential Advisors & 4640 & 4640 & & 3 \\
\hline $\mathrm{IIIb}$ & Retail Salespersons & 4760 & 4760 & & 321 \\
\hline IIIlb & $\begin{array}{l}\text { Security Guards And Gaming Surveillance } \\
\text { Officers }\end{array}$ & 3930 & 3930 & & 66 \\
\hline $\mathrm{IIIlb}$ & Telemarketers & 4940 & 4940 & & 17 \\
\hline \multirow[t]{2}{*}{ IIIlb } & Transportation Attendants, Except Flight & & & & \\
\hline & Attendants & 9415 & 9415 & & 3 \\
\hline $\mathrm{IIIb}$ & Transportation Security Screeners & 3945 & 3945 & & 3 \\
\hline $\mathrm{IIIb}$ & Ushers, Lobby Attendants, And Ticket Takers & 4420 & 4420 & & 3 \\
\hline IIIlb & $\begin{array}{l}\text { Veterinary Assistants And Laboratory Animal } \\
\text { Caretakers }\end{array}$ & 3648 & 3648 & & 4 \\
\hline $\mathrm{IIIb}$ & Waiters And Waitresses & 4110 & 4110 & & 274 \\
\hline \multirow[t]{2}{*}{ IIIlb } & Wholesale And Retail Buyers, Except Farm & & & & \\
\hline & Products & 520 & 520 & & 14 \\
\hline IVa & Appraisers And Assessors Of Real Estate & 810 & 810 & & 2 \\
\hline IVa & Artists And Related Workers & 2600 & 2600 & & 2 \\
\hline IVa & Automotive Body And Related Repairers & 7150 & 7150 & & 1 \\
\hline \multirow[t]{2}{*}{ IVa } & Automotive Service Technicians And & & & & \\
\hline & Mechanics & 7200 & 7200 & & 13 \\
\hline IVa & Bakers & 7800 & 7800 & & 3 \\
\hline IVa & Barbers & 4500 & 4500 & & 2 \\
\hline IVa & Bartenders & 4040 & 4040 & & 7 \\
\hline \multirow[t]{2}{*}{ IVa } & Bookkeeping, Accounting, And Auditing & & & & \\
\hline & Clerks & 5120 & 5120 & & 7 \\
\hline IVa & $\begin{array}{l}\text { Brickmasons, Blockmasons, Stonemasons, And } \\
\text { Reinforcing Iron And Rebar Workers }\end{array}$ & 6220 & 6220 & & 2 \\
\hline IVa & Cabinetmakers And Bench Carpenters & 8500 & 8500 & & 4 \\
\hline IVa & Carpenters & 6230 & 6230 & & 20 \\
\hline IVa & $\begin{array}{l}\text { Carpet, Floor, And Tile Installers And } \\
\text { Finishers }\end{array}$ & 6240 & 6240 & & 5 \\
\hline IVa & Cashiers & 4720 & 4720 & & 1 \\
\hline IVa & Chefs And Head Cooks & 4000 & 4000 & & 2 \\
\hline IVa & Childcare Workers & 4600 & 4600 & & 4 \\
\hline IVa & Cleaners Of Vehicles And Equipment & 9610 & 9610 & & 2 \\
\hline \multirow[t]{2}{*}{ IVa } & Computer, Automated Teller, And Office & & & & \\
\hline & Machine Repairers & 7010 & 7010 & & 1 \\
\hline IVa & Construction And Building Inspectors & 6660 & 6660 & & 1 \\
\hline \multirow[t]{2}{*}{ IVa } & $\begin{array}{l}\text { Construction Equipment Operators, Except } \\
\text { Paving, Surfacing, And Tamping Equipment }\end{array}$ & & & & \\
\hline & Operators & 6320 & 6320 & & 4 \\
\hline
\end{tabular}




\begin{tabular}{|c|c|c|c|c|c|}
\hline Class & Occupational Title (ACS 2012) & $\begin{array}{l}\text { ACS } \\
2012\end{array}$ & $\begin{array}{r}\text { GSS } \\
\text { occ10 }\end{array}$ & $\begin{array}{l}\text { Recoded } \\
\text { to ACS }\end{array}$ & $\begin{array}{r}\text { GSS } \\
2004- \\
2016\end{array}$ \\
\hline IVa & Construction Laborers & 6260 & 6260 & & 13 \\
\hline IVa & Construction Managers & 220 & 220 & & 36 \\
\hline IVa & Cost Estimators & 600 & 600 & & 1 \\
\hline IVa & Counter And Rental Clerks & 4740 & 4740 & & 2 \\
\hline IVa & Couriers And Messengers & 5510 & 5510 & & 1 \\
\hline IVa & Credit Counselors And Loan Officers & 910 & 910 & & 1 \\
\hline IVa & Customer Service Representatives & 5240 & 5240 & & 2 \\
\hline IVa & Designers & 2630 & 2630 & & 5 \\
\hline IVa & $\begin{array}{l}\text { Diagnostic Related Technologists And } \\
\text { Technicians }\end{array}$ & 3320 & 3320 & & 1 \\
\hline IVa & $\begin{array}{l}\text { Door-To-Door Sales Workers, News And } \\
\text { Street Vendors, And Related Workers }\end{array}$ & 4950 & 4950 & & 2 \\
\hline IVa & $\begin{array}{l}\text { Dredge, Excavating, And Loading Machine } \\
\text { Operators }\end{array}$ & 9520 & 9520 & & 1 \\
\hline IVa & Driver/Sales Workers And Truck Drivers & 9130 & 9130 & & 12 \\
\hline IVa & $\begin{array}{l}\text { Drywall Installers, Ceiling Tile Installers, And } \\
\text { Tapers }\end{array}$ & 6330 & 6330 & & 1 \\
\hline IVa & $\begin{array}{l}\text { Electric Motor, Power Tool, And Related } \\
\text { Repairers }\end{array}$ & 7040 & 7040 & & 1 \\
\hline IVa & Electricians & 6355 & 6355 & & 1 \\
\hline IVa & $\begin{array}{l}\text { Electronic Home Entertainment Equipment } \\
\text { Installers And Repairers }\end{array}$ & 7120 & 7120 & & 1 \\
\hline IVa & $\begin{array}{l}\text { First-Line Supervisors Of Construction Trades } \\
\text { And Extraction Workers }\end{array}$ & 6200 & 6200 & & 15 \\
\hline IVa & $\begin{array}{l}\text { First-Line Supervisors Of Housekeeping And } \\
\text { Janitorial Workers }\end{array}$ & 4200 & 4200 & & 4 \\
\hline IVa & $\begin{array}{l}\text { First-Line Supervisors Of Landscaping, Lawn } \\
\text { Service, And Groundskeeping Workers }\end{array}$ & 4210 & 4210 & & 5 \\
\hline IVa & $\begin{array}{l}\text { First-Line Supervisors Of Non-Retail Sales } \\
\text { Workers }\end{array}$ & 4710 & 4710 & & 13 \\
\hline IVa & $\begin{array}{l}\text { First-Line Supervisors Of Office And } \\
\text { Administrative Support Workers }\end{array}$ & 5000 & 5000 & & 7 \\
\hline IVa & $\begin{array}{l}\text { First-Line Supervisors Of Personal Service } \\
\text { Workers }\end{array}$ & 4320 & 4320 & & 3 \\
\hline IVa & $\begin{array}{l}\text { First-Line Supervisors Of Production And } \\
\text { Operating Workers }\end{array}$ & 7700 & 7700 & & 2 \\
\hline IVa & First-Line Supervisors Of Retail Sales Workers & 4700 & 4700 & & 49 \\
\hline IVa & $\begin{array}{l}\text { First-Line Supervisors Of Food Preparation } \\
\text { And Serving Workers }\end{array}$ & 4010 & 4010 & & 5 \\
\hline IVa & Fishing And Hunting Workers & 6100 & 6100 & & 3 \\
\hline IVa & Food Service Managers & 310 & 310 & & 31 \\
\hline IVa & Grounds Maintenance Workers & 4250 & 4250 & & 4 \\
\hline IVa & Hairdressers, Hairstylists, And Cosmetologists & 4510 & 4510 & & 19 \\
\hline IVa & $\begin{array}{l}\text { Heating, Air Conditioning, And Refrigeration } \\
\text { Mechanics And Installers }\end{array}$ & 7315 & 7315 & & 2 \\
\hline IVa & Human Resources Workers & 630 & 630 & & 1 \\
\hline IVa & Industrial Truck And Tractor Operators & 9600 & 9600 & & 1 \\
\hline
\end{tabular}




\begin{tabular}{|c|c|c|c|c|c|}
\hline Class & Occupational Title (ACS 2012) & $\begin{array}{l}\text { ACS } \\
2012\end{array}$ & $\begin{array}{r}\text { GSS } \\
\text { occ10 }\end{array}$ & $\begin{array}{l}\text { Recoded } \\
\text { to ACS }\end{array}$ & $\begin{array}{r}\text { GSS } \\
2004- \\
2016\end{array}$ \\
\hline IVa & Insulation Workers & 6400 & 6400 & & 1 \\
\hline IVa & Insurance Sales Agents & 4810 & 4810 & & 7 \\
\hline IVa & Janitors And Building Cleaners & 4220 & 4220 & & 3 \\
\hline IVa & $\begin{array}{l}\text { Laborers And Freight, Stock, And Material } \\
\text { Movers, Hand }\end{array}$ & 9620 & 9620 & & 1 \\
\hline IVa & Laundry And Dry-Cleaning Workers & 8300 & 8300 & & 3 \\
\hline IVa & Locksmiths And Safe Repairers & 7540 & 7540 & & 1 \\
\hline IVa & Lodging Managers & 340 & 340 & & 3 \\
\hline IVa & Logging Workers & 6130 & 6130 & & 2 \\
\hline $\mathrm{IVa}$ & Machinists & 8030 & 8030 & & 1 \\
\hline IVa & Maids And Housekeeping Cleaners & 4230 & 4230 & & 7 \\
\hline IVa & Massage Therapists & 3630 & 3630 & & 1 \\
\hline IVa & $\begin{array}{l}\text { Miscellaneous Agricultural Workers, } \\
\text { Including Animal Breeders }\end{array}$ & 6050 & 6050 & & 3 \\
\hline IVa & Miscellaneous Legal Support Workers & 2160 & 2160 & & 2 \\
\hline IVa & Miscellaneous Personal Appearance Workers & 4520 & 4520 & & 1 \\
\hline IVa & Miscellaneous Production Workers, Including & & & & \\
\hline IVa & $\begin{array}{l}\text { Semiconductor Processors } \\
\text { Miscellaneous Vehicle And Mobile Equipment }\end{array}$ & 8965 & 8965 & & 1 \\
\hline & Mechanics, Installers, And Repairers & 7260 & 7260 & & 1 \\
\hline IVa & Musicians, Singers, And Related Workers & 2750 & 2750 & & 3 \\
\hline IVa & Nonfarm Animal Caretakers & 4350 & 4350 & & 2 \\
\hline IVa & Nursing, Psychiatric, And Home Health Aides & 3600 & 3600 & & 1 \\
\hline IVa & Office Clerks, General & 5860 & 5860 & & 1 \\
\hline IVa & Other Teachers And Instructors & 2340 & 2340 & & 2 \\
\hline IVa & Painters And Paperhangers & 6420 & 6420 & & 6 \\
\hline IVa & Painting Workers & 8810 & 8810 & & 2 \\
\hline IVa & Paralegals And Legal Assistants & 2145 & 2145 & & 1 \\
\hline IVa & Payroll And Timekeeping Clerks & 5140 & 5140 & & 1 \\
\hline IVa & Personal Care Aides & 4610 & 4610 & & 2 \\
\hline IVa & Pest Control Workers & 4240 & 4240 & & 1 \\
\hline IVa & Photographers & 2910 & 2910 & & 1 \\
\hline IVa & $\begin{array}{l}\text { Pipelayers, Plumbers, Pipefitters, And } \\
\text { Steamfitters }\end{array}$ & 6440 & 6440 & & 7 \\
\hline IVa & $\begin{array}{l}\text { Precision Instrument And Equipment } \\
\text { Repairers }\end{array}$ & 7430 & 7430 & & 1 \\
\hline IVa & Printing Press Operators & 8255 & 8255 & & 2 \\
\hline IVa & Property, Real Estate, And Community & & & & \\
\hline & Association Managers & 410 & 410 & & 5 \\
\hline IVa & Pumping Station Operators & 9650 & 9650 & & 1 \\
\hline IVa & Real Estate Brokers And Sales Agents & 4920 & 4920 & & 14 \\
\hline IVa & Recreation And Fitness Workers & 4620 & 4620 & & 2 \\
\hline IVa & Retail Salespersons & 4760 & 4760 & & 22 \\
\hline IVa & Roofers & 6515 & 6515 & & 2 \\
\hline IVa & Sales And Related Workers, All Other & 4965 & 4965 & & 1 \\
\hline
\end{tabular}




\begin{tabular}{|c|c|c|c|c|c|}
\hline Class & Occupational Title (ACS 2012) & $\begin{array}{l}\text { ACS } \\
2012 \\
\end{array}$ & $\begin{array}{r}\text { GSS } \\
\text { occ10 }\end{array}$ & $\begin{array}{l}\text { Recoded } \\
\text { to ACS }\end{array}$ & $\begin{array}{r}\text { GSS } \\
2004- \\
2016\end{array}$ \\
\hline IVa & Sales Representatives, Services, All Other & 4840 & 4840 & & 2 \\
\hline \multirow[t]{2}{*}{ IVa } & Sales Representatives, Wholesale And & & & & \\
\hline & Manufacturing & 4850 & 4850 & & 10 \\
\hline IVa & Secretaries And Administrative Assistants & 5700 & 5700 & & 8 \\
\hline IVa & Stationary Engineers And Boiler Operators & 8610 & 8610 & & 1 \\
\hline IVa & Structural Iron And Steel Workers & 6530 & 6530 & & 1 \\
\hline IVa & Structural Metal Fabricators And Fitters & 7740 & 7740 & & 2 \\
\hline \multirow[t]{2}{*}{ IVa } & Supervisors Of Transportation And Material & & & & \\
\hline & Moving Workers & 9000 & 9000 & & 3 \\
\hline IVa & Surveying And Mapping Technicians & 1560 & 1560 & & 1 \\
\hline IVa & Tailors, Dressmakers, And Sewers & 8350 & 8350 & & 2 \\
\hline IVa & Taxi Drivers And Chauffeurs & 9140 & 9140 & & 2 \\
\hline \multirow[t]{2}{*}{ IVa } & Telecommunications Line Installers And & & & & \\
\hline & Repairers & 7420 & 7420 & & 1 \\
\hline IVa & Travel Agents & 4830 & 4830 & & 1 \\
\hline IVa & Waiters And Waitresses & 4110 & 4110 & & 1 \\
\hline IVa & Welding, Soldering, And Brazing Workers & 8140 & 8140 & & 3 \\
\hline \multirow[t]{2}{*}{ IVa } & Wholesale And Retail Buyers, Except Farm & & & & \\
\hline & Products & 520 & 520 & & 2 \\
\hline $\mathrm{IVb}$ & Actors & 2700 & 2700 & & 1 \\
\hline $\mathrm{IVb}$ & Advertising Sales Agents & 4800 & 4800 & & 2 \\
\hline $\mathrm{IVb}$ & Agricultural Inspectors & 6010 & 6010 & & 1 \\
\hline $\mathrm{IVb}$ & Appraisers And Assessors Of Real Estate & 810 & 810 & & 4 \\
\hline $\mathrm{IVb}$ & Artists And Related Workers & 2600 & 2600 & & 20 \\
\hline \multirow[t]{2}{*}{$\mathrm{IVb}$} & Athletes, Coaches, Umpires, And Related & & & & \\
\hline & Workers & 2720 & 2720 & & 5 \\
\hline $\mathrm{IVb}$ & Automotive Body And Related Repairers & 7150 & 7150 & & 4 \\
\hline $\mathrm{IVb}$ & Automotive Glass Installers And Repairers & 7160 & 7160 & & 2 \\
\hline \multirow[t]{2}{*}{$\mathrm{IVb}$} & Automotive Service Technicians And & & & & \\
\hline & Mechanics & 7200 & 7200 & & 15 \\
\hline $\mathrm{IVb}$ & Barbers & 4500 & 4500 & & 6 \\
\hline $\mathrm{IVb}$ & Bartenders & 4040 & 4040 & & 1 \\
\hline $\mathrm{IVb}$ & Billing And Posting Clerks & 5110 & 5110 & & 1 \\
\hline \multirow[t]{2}{*}{$\mathrm{IVb}$} & Bookkeeping, Accounting, And Auditing & & & & \\
\hline & Clerks & 5120 & 5120 & & 10 \\
\hline \multirow[t]{2}{*}{$\mathrm{IVb}$} & Brickmasons, Blockmasons, Stonemasons, And & & & & \\
\hline & Reinforcing Iron And Rebar Workers & 6220 & 6220 & & 5 \\
\hline \multirow[t]{4}{*}{$\mathrm{IVb}$} & Broadcast And Sound Engineering & & & & \\
\hline & Technicians And Radio Operators, And Media & & & & \\
\hline & And Communication Equipment Workers, All & & & & \\
\hline & Other & 2900 & 2900 & & 2 \\
\hline \multirow[t]{2}{*}{$\mathrm{IVb}$} & Bus And Truck Mechanics And Diesel Engine & & & & \\
\hline & Specialists & 7210 & 7210 & & 1 \\
\hline $\mathrm{IVb}$ & Bus Drivers & 9120 & 9120 & & 1 \\
\hline \multirow[t]{2}{*}{$\mathrm{IVb}$} & Buyers And Purchasing Agents, Farm & & & & \\
\hline & Products & 510 & 510 & & 2 \\
\hline
\end{tabular}




\begin{tabular}{|c|c|c|c|c|c|}
\hline Class & Occupational Title (ACS 2012) & $\begin{array}{l}\text { ACS } \\
2012 \\
\end{array}$ & $\begin{array}{r}\text { GSS } \\
\text { occ10 } \\
\end{array}$ & $\begin{array}{l}\text { Recoded } \\
\text { to ACS }\end{array}$ & $\begin{array}{r}\text { GSS } \\
2004- \\
2016 \\
\end{array}$ \\
\hline $\mathrm{IVb}$ & Cabinetmakers And Bench Carpenters & 8500 & 8500 & & 6 \\
\hline $\mathrm{IVb}$ & Carpenters & 6230 & 6230 & & 32 \\
\hline $\mathrm{IVb}$ & $\begin{array}{l}\text { Carpet, Floor, And Tile Installers And } \\
\text { Finishers }\end{array}$ & 6240 & 6240 & & 3 \\
\hline $\mathrm{IVb}$ & Cashiers & 4720 & 4720 & & 3 \\
\hline $\mathrm{IVb}$ & $\begin{array}{l}\text { Cement Masons, Concrete Finishers, And } \\
\text { Terrazzo Workers }\end{array}$ & 6250 & 6250 & & 1 \\
\hline $\mathrm{IVb}$ & Chefs And Head Cooks & 4000 & 4000 & & 3 \\
\hline $\mathrm{IVb}$ & Childcare Workers & 4600 & 4600 & & 64 \\
\hline $\mathrm{IVb}$ & Cleaners Of Vehicles And Equipment & 9610 & 9610 & & 2 \\
\hline $\mathrm{IVb}$ & Computer Support Specialists & 1050 & 1050 & & 1 \\
\hline $\mathrm{IVb}$ & $\begin{array}{l}\text { Computer, Automated Teller, And Office } \\
\text { Machine Repairers } \\
\text { Construction Equipment Operators, Except } \\
\text { Paving, Surfacing, And Tamping Equipment }\end{array}$ & 7010 & 7010 & & 5 \\
\hline & Operators & 6320 & 6320 & & 3 \\
\hline $\mathrm{IVb}$ & Construction Laborers & 6260 & 6260 & & 38 \\
\hline $\mathrm{IVb}$ & Construction Managers & 220 & 220 & & 27 \\
\hline $\mathrm{IVb}$ & Cooks & 4020 & 4020 & & 1 \\
\hline $\mathrm{IVb}$ & Counter And Rental Clerks & 4740 & 4740 & & 1 \\
\hline $\mathrm{IVb}$ & Couriers And Messengers & 5510 & 5510 & & 2 \\
\hline $\mathrm{IVb}$ & Crane And Tower Operators & 9510 & 9510 & & 1 \\
\hline $\mathrm{IVb}$ & Credit Counselors And Loan Officers & 910 & 910 & & 1 \\
\hline $\mathrm{IVb}$ & Dancers And Choreographers & 2740 & 2740 & & 1 \\
\hline $\mathrm{IVb}$ & Dental Hygienists & 3310 & 3310 & & 2 \\
\hline $\mathrm{IVb}$ & Designers & 2630 & 2630 & & 25 \\
\hline $\mathrm{IVb}$ & Detectives And Criminal Investigators & 3820 & 3820 & & 1 \\
\hline $\mathrm{IVb}$ & Dishwashers & 4140 & 4140 & & 1 \\
\hline $\mathrm{IVb}$ & $\begin{array}{l}\text { Door-To-Door Sales Workers, News And } \\
\text { Street Vendors, And Related Workers }\end{array}$ & 4950 & 4950 & & 5 \\
\hline $\mathrm{IVb}$ & Drafters & 1540 & 1540 & & 2 \\
\hline $\mathrm{IVb}$ & $\begin{array}{l}\text { Dredge, Excavating, And Loading Machine } \\
\text { Operators }\end{array}$ & 9520 & 9520 & & 1 \\
\hline $\mathrm{IVb}$ & Driver/Sales Workers And Truck Drivers & 9130 & 9130 & & 36 \\
\hline $\mathrm{IVb}$ & $\begin{array}{l}\text { Drywall Installers, Ceiling Tile Installers, And } \\
\text { Tapers }\end{array}$ & 6330 & 6330 & & 2 \\
\hline $\mathrm{IVb}$ & Electricians & 6355 & 6355 & & 6 \\
\hline $\mathrm{IVb}$ & $\begin{array}{l}\text { Electronic Home Entertainment Equipment } \\
\text { Installers And Repairers }\end{array}$ & 7120 & 7120 & & 2 \\
\hline $\mathrm{IVb}$ & $\begin{array}{l}\text { Entertainers And Performers, Sports And } \\
\text { Related Workers, All Other }\end{array}$ & 2760 & 2760 & & 3 \\
\hline $\mathrm{IVb}$ & Fence Erectors & 6710 & 6710 & & 1 \\
\hline $\mathrm{IVb}$ & $\begin{array}{l}\text { First-Line Supervisors Of Construction Trades } \\
\text { And Extraction Workers }\end{array}$ & 6200 & 6200 & & 13 \\
\hline $\mathrm{IVb}$ & $\begin{array}{l}\text { First-Line Supervisors Of Housekeeping And } \\
\text { Janitorial Workers }\end{array}$ & 4200 & 4200 & & 3 \\
\hline
\end{tabular}




\begin{tabular}{|c|c|c|c|c|c|}
\hline Class & Occupational Title (ACS 2012) & $\begin{array}{l}\text { ACS } \\
2012\end{array}$ & $\begin{array}{r}\text { GSS } \\
\text { occ10 }\end{array}$ & $\begin{array}{l}\text { Recoded } \\
\text { to ACS }\end{array}$ & $\begin{array}{r}\text { GSS } \\
2004- \\
2016 \\
\end{array}$ \\
\hline $\mathrm{IVb}$ & $\begin{array}{l}\text { First-Line Supervisors Of Landscaping, Lawn } \\
\text { Service, And Groundskeeping Workers }\end{array}$ & 4210 & 4210 & & 9 \\
\hline $\mathrm{IVb}$ & $\begin{array}{l}\text { First-Line Supervisors Of Mechanics, } \\
\text { Installers, And Repairers }\end{array}$ & 7000 & 7000 & & 1 \\
\hline $\mathrm{IVb}$ & $\begin{array}{l}\text { First-Line Supervisors Of Non-Retail Sales } \\
\text { Workers }\end{array}$ & 4710 & 4710 & & 5 \\
\hline $\mathrm{IVb}$ & $\begin{array}{l}\text { First-Line Supervisors Of Personal Service } \\
\text { Workers }\end{array}$ & 4320 & 4320 & & 1 \\
\hline $\mathrm{IVb}$ & $\begin{array}{l}\text { First-Line Supervisors Of Production And } \\
\text { Operating Workers }\end{array}$ & 7700 & 7700 & & 1 \\
\hline $\mathrm{IVb}$ & First-Line Supervisors Of Retail Sales Workers & 4700 & 4700 & & 39 \\
\hline $\mathrm{IVb}$ & $\begin{array}{l}\text { First-Line Supervisors Of Food Preparation } \\
\text { And Serving Workers }\end{array}$ & 4010 & 4010 & & 4 \\
\hline $\mathrm{IVb}$ & Fishing And Hunting Workers & 6100 & 6100 & & 7 \\
\hline $\mathrm{IVb}$ & Furniture Finishers & 8510 & 8510 & & 1 \\
\hline $\mathrm{IVb}$ & Grounds Maintenance Workers & 4250 & 4250 & & 26 \\
\hline $\mathrm{IVb}$ & Hairdressers, Hairstylists, And Cosmetologists & 4510 & 4510 & & 53 \\
\hline $\mathrm{IVb}$ & $\begin{array}{l}\text { Health Practitioner Support Technologists } \\
\text { And Technicians }\end{array}$ & 3420 & 3420 & & 1 \\
\hline $\mathrm{IVb}$ & $\begin{array}{l}\text { Heating, Air Conditioning, And Refrigeration } \\
\text { Mechanics And Installers }\end{array}$ & 7315 & 7315 & & 5 \\
\hline $\mathrm{IVb}$ & Highway Maintenance Workers & 6730 & 6730 & & 1 \\
\hline $\mathrm{IVb}$ & Home Appliance Repairers & 7320 & 7320 & & 1 \\
\hline $\mathrm{IVb}$ & Human Resources Workers & 630 & 630 & & 6 \\
\hline $\mathrm{IVb}$ & $\begin{array}{l}\text { Industrial And Refractory Machinery } \\
\text { Mechanics }\end{array}$ & 7330 & 7330 & & 1 \\
\hline $\mathrm{IVb}$ & Industrial Truck And Tractor Operators & 9600 & 9600 & & 1 \\
\hline $\mathrm{IVb}$ & $\begin{array}{l}\text { Inspectors, Testers, Sorters, Samplers, And } \\
\text { Weighers }\end{array}$ & 8740 & 8740 & & 3 \\
\hline $\mathrm{IVb}$ & Insurance Sales Agents & 4810 & 4810 & & 12 \\
\hline $\mathrm{IVb}$ & Interviewers, Except Eligibility And Loan & 5310 & 5310 & & 1 \\
\hline $\mathrm{IVb}$ & Janitors And Building Cleaners & 4220 & 4220 & & 15 \\
\hline $\mathrm{IVb}$ & $\begin{array}{l}\text { Jewelers And Precious Stone And Metal } \\
\text { Workers }\end{array}$ & 8750 & 8750 & & 1 \\
\hline $\mathrm{IVb}$ & $\begin{array}{l}\text { Laborers And Freight, Stock, And Material } \\
\text { Movers, Hand }\end{array}$ & 9620 & 9620 & & 8 \\
\hline $\mathrm{IVb}$ & Laundry And Dry-Cleaning Workers & 8300 & 8300 & & 1 \\
\hline $\mathrm{IVb}$ & $\begin{array}{l}\text { Licensed Practical And Licensed Vocational } \\
\text { Nurses }\end{array}$ & 3500 & 3500 & & 2 \\
\hline $\mathrm{IVb}$ & Loan Interviewers And Clerks & 5330 & 5330 & & 1 \\
\hline $\mathrm{IVb}$ & Locksmiths And Safe Repairers & 7540 & 7540 & & 2 \\
\hline $\mathrm{IVb}$ & Lodging Managers & 340 & 340 & & 2 \\
\hline $\mathrm{IVb}$ & Logging Workers & 6130 & 6130 & & 4 \\
\hline $\mathrm{IVb}$ & Machinists & 8030 & 8030 & & 1 \\
\hline $\mathrm{IVb}$ & Maids And Housekeeping Cleaners & 4230 & 4230 & & 74 \\
\hline $\mathrm{IVb}$ & Maintenance And Repair Workers, General & 7340 & 7340 & & 2 \\
\hline $\mathrm{IVb}$ & Massage Therapists & 3630 & 3630 & & 11 \\
\hline
\end{tabular}




\begin{tabular}{|c|c|c|c|c|c|}
\hline Class & Occupational Title (ACS 2012) & $\begin{array}{l}\text { ACS } \\
2012 \\
\end{array}$ & $\begin{array}{r}\text { GSS } \\
\text { occ10 }\end{array}$ & $\begin{array}{l}\text { Recoded } \\
\text { to ACS }\end{array}$ & $\begin{array}{r}\text { GSS } \\
2004- \\
2016 \\
\end{array}$ \\
\hline $\mathrm{IVb}$ & Medical Assistants & 3645 & 3645 & & 1 \\
\hline \multirow[t]{2}{*}{$\mathrm{IVb}$} & Medical, Dental, And Ophthalmic Laboratory & & & & \\
\hline & Technicians & 8760 & 8760 & & 1 \\
\hline $\mathrm{IVb}$ & Meeting, Convention, And Event Planners & 725 & 725 & & 1 \\
\hline $\mathrm{IVb}$ & $\begin{array}{l}\text { Miscellaneous Agricultural Workers, } \\
\text { Including Animal Breeders }\end{array}$ & 6050 & 6050 & & 5 \\
\hline $\mathrm{IVb}$ & Miscellaneous Assemblers And Fabricators & 7750 & 7750 & & 5 \\
\hline \multirow[t]{2}{*}{$\mathrm{IVb}$} & $\begin{array}{l}\text { Miscellaneous Installation, Maintenance, And } \\
\text { Repair Workers, Including Wind Turbine }\end{array}$ & & & & \\
\hline & Service Technicians & 7630 & 7630 & & 3 \\
\hline $\mathrm{IVb}$ & Miscellaneous Legal Support Workers & 2160 & 2160 & & 5 \\
\hline $\mathrm{IVb}$ & $\begin{array}{l}\text { Miscellaneous Material Moving Workers, } \\
\text { Including Mine Shuttle Car Operators, And }\end{array}$ & & & & \\
\hline $\mathrm{IVb}$ & $\begin{array}{l}\text { Tank Car, Truck, And Ship Loaders } \\
\text { Miscellaneous Media And Communication }\end{array}$ & 9750 & 9750 & & 1 \\
\hline \multirow[t]{2}{*}{$\mathrm{IVb}$} & $\begin{array}{l}\text { Workers } \\
\text { Miscellaneous Office And Administrative } \\
\text { Support Workers, Including Desktop }\end{array}$ & 2860 & 2860 & & 5 \\
\hline & Publishers & 5940 & 5940 & & 2 \\
\hline $\mathrm{IVb}$ & Miscellaneous Personal Appearance Workers & 4520 & 4520 & & 7 \\
\hline $\mathrm{IVb}$ & Miscellaneous Production Workers, Including & & & & \\
\hline & Semiconductor Processors & 8965 & 8965 & & 2 \\
\hline $\mathrm{IVb}$ & $\begin{array}{l}\text { Miscellaneous Woodworkers, Including } \\
\text { Model Makers And Patternmakers }\end{array}$ & 8550 & 8550 & & 1 \\
\hline $\mathrm{IVb}$ & Motion Picture Projectionists & 4410 & 4410 & & 1 \\
\hline $\mathrm{IVb}$ & Musicians, Singers, And Related Workers & 2750 & 2750 & & 12 \\
\hline $\mathrm{IVb}$ & Nonfarm Animal Caretakers & 4350 & 4350 & & 5 \\
\hline $\mathrm{IVb}$ & Nursing, Psychiatric, And Home Health Aides & 3600 & 3600 & & 12 \\
\hline $\mathrm{IVb}$ & Office Clerks, General & 5860 & 5860 & & 2 \\
\hline $\mathrm{IVb}$ & Other Teachers And Instructors & 2340 & 2340 & & 22 \\
\hline $\mathrm{IVb}$ & $\begin{array}{l}\text { Packaging And Filling Machine Operators } \\
\text { And Tenders }\end{array}$ & 8800 & 8800 & & 1 \\
\hline $\mathrm{IVb}$ & Packers And Packagers, Hand & 9640 & 9640 & & 1 \\
\hline $\mathrm{IVb}$ & Painters And Paperhangers & 6420 & 6420 & & 17 \\
\hline $\mathrm{IVb}$ & Painters And Paperhangers & 6420 & 6430 & $x$ & 1 \\
\hline $\mathrm{IVb}$ & Paralegals And Legal Assistants & 2145 & 2145 & & 1 \\
\hline $\mathrm{IVb}$ & Parts Salespersons & 4750 & 4750 & & 1 \\
\hline $\mathrm{IVb}$ & $\begin{array}{l}\text { Paving, Surfacing, And Tamping Equipment } \\
\text { Operators }\end{array}$ & 6300 & 6300 & & 1 \\
\hline $\mathrm{IVb}$ & Personal Care Aides & 4610 & 4610 & & 24 \\
\hline $\mathrm{IVb}$ & Personal Care And Service Workers, All Other & 4650 & 4650 & & 4 \\
\hline $\mathrm{IVb}$ & Pest Control Workers & 4240 & 4240 & & 1 \\
\hline $\mathrm{IVb}$ & Photographers & 2910 & 2910 & & 11 \\
\hline \multirow[t]{2}{*}{$\mathrm{IVb}$} & Pipelayers, Plumbers, Pipefitters, And & & & & \\
\hline & $\begin{array}{l}\text { Steamfitters } \\
\text { Precision Instrument And Equipment }\end{array}$ & 6440 & 6440 & & 8 \\
\hline $\mathrm{IVb}$ & Repairers & 7430 & 7430 & & 1 \\
\hline
\end{tabular}




\begin{tabular}{|c|c|c|c|c|c|}
\hline Class & Occupational Title (ACS 2012) & $\begin{array}{l}\text { ACS } \\
2012 \\
\end{array}$ & $\begin{array}{r}\text { GSS } \\
\text { occ10 } \\
\end{array}$ & $\begin{array}{l}\text { Recoded } \\
\text { to ACS }\end{array}$ & $\begin{array}{r}\text { GSS } \\
2004- \\
2016 \\
\end{array}$ \\
\hline $\mathrm{IVb}$ & Preschool And Kindergarten Teachers & 2300 & 2300 & & 1 \\
\hline $\mathrm{IVb}$ & Printing Press Operators & 8255 & 8255 & & 4 \\
\hline $\mathrm{IVb}$ & Private Detectives And Investigators & 3910 & 3910 & & 1 \\
\hline $\mathrm{IVb}$ & Production, Planning, And Expediting Clerks & 5600 & 5600 & & 1 \\
\hline $\mathrm{IVb}$ & Property, Real Estate, And Community & & & & \\
\hline \multirow[b]{2}{*}{$\mathrm{IVb}$} & Association Managers & 410 & 410 & & 9 \\
\hline & $\begin{array}{l}\text { Radio And Telecommunications Equipment } \\
\text { Installers And Repairers }\end{array}$ & 7020 & 7020 & & 1 \\
\hline $\mathrm{IVb}$ & Real Estate Brokers And Sales Agents & 4920 & 4920 & & 53 \\
\hline $\mathrm{IVb}$ & Recreation And Fitness Workers & 4620 & 4620 & & 4 \\
\hline $\mathrm{IVb}$ & Refuse And Recyclable Material Collectors & 9720 & 9720 & & 3 \\
\hline $\mathrm{IVb}$ & Religious Workers, All Other & 2060 & 2060 & & 1 \\
\hline $\mathrm{IVb}$ & Retail Salespersons & 4760 & 4760 & & 25 \\
\hline $\mathrm{IVb}$ & Roofers & 6515 & 6515 & & 2 \\
\hline $\mathrm{IVb}$ & Sales And Related Workers, All Other & 4965 & 4965 & & 2 \\
\hline $\mathrm{IVb}$ & Sales Representatives, Services, All Other & 4840 & 4840 & & 3 \\
\hline $\mathrm{IVb}$ & Sales Representatives, Wholesale And & & & & \\
\hline & Manufacturing & 4850 & 4850 & & 14 \\
\hline $\mathrm{IVb}$ & Secretaries And Administrative Assistants & 5700 & 5700 & & 7 \\
\hline $\mathrm{IVb}$ & $\begin{array}{l}\text { Security Guards And Gaming Surveillance } \\
\text { Officers }\end{array}$ & 3930 & 3930 & & 1 \\
\hline $\mathrm{IVb}$ & Sheet Metal Workers & 6520 & 6520 & & 1 \\
\hline $\mathrm{IVb}$ & Shipping, Receiving, And Traffic Clerks & 5610 & 5610 & & 1 \\
\hline $\mathrm{IVb}$ & Shoe And Leather Workers & 8330 & 8330 & & 2 \\
\hline $\mathrm{IVb}$ & Stock Clerks And Order Fillers & 5620 & 5620 & & 1 \\
\hline $\mathrm{IVb}$ & $\begin{array}{l}\text { Supervisors Of Transportation And Material } \\
\text { Moving Workers }\end{array}$ & 9000 & 9000 & & 1 \\
\hline $\mathrm{IVb}$ & Tailors, Dressmakers, And Sewers & 8350 & 8350 & & 11 \\
\hline $\mathrm{IVb}$ & Tax Preparers & 940 & 940 & & 7 \\
\hline $\mathrm{IVb}$ & Taxi Drivers And Chauffeurs & 9140 & 9140 & & 9 \\
\hline $\mathrm{IVb}$ & Teacher Assistants & 2540 & 2540 & & 1 \\
\hline $\mathrm{IVb}$ & $\begin{array}{l}\text { Telecommunications Line Installers And } \\
\text { Repairers }\end{array}$ & 7420 & 7420 & & 1 \\
\hline $\mathrm{IVb}$ & $\begin{array}{l}\text { Television, Video, And Motion Picture Camera } \\
\text { Operators And Editors }\end{array}$ & 2920 & 2920 & & 2 \\
\hline $\mathrm{IVb}$ & Travel Agents & 4830 & 4830 & & 4 \\
\hline $\mathrm{IVb}$ & Upholsterers & 8450 & 8450 & & 3 \\
\hline $\mathrm{IVb}$ & Waiters And Waitresses & 4110 & 4110 & & 1 \\
\hline $\mathrm{IVb}$ & Web Developers & 1030 & 1030 & & 3 \\
\hline $\mathrm{IVb}$ & Welding, Soldering, And Brazing Workers & 8140 & 8140 & & 8 \\
\hline $\mathrm{IVb}$ & $\begin{array}{l}\text { Wholesale And Retail Buyers, Except Farm } \\
\text { Products }\end{array}$ & 520 & 520 & & 2 \\
\hline $\mathrm{IVb}$ & $\begin{array}{l}\text { Woodworking Machine Setters, Operators, } \\
\text { And Tenders, Except Sawing }\end{array}$ & 8540 & 8540 & & 1 \\
\hline IVc & $\begin{array}{l}\text { Farmers, Ranchers, And Other Agricultural } \\
\text { Managers }\end{array}$ & 205 & 205 & & 82 \\
\hline
\end{tabular}




\begin{tabular}{|c|c|c|c|c|c|}
\hline Class & Occupational Title (ACS 2012) & $\begin{array}{l}\text { ACS } \\
2012 \\
\end{array}$ & $\begin{array}{r}\text { GSS } \\
\text { occ10 } \\
\end{array}$ & $\begin{array}{l}\text { Recoded } \\
\text { to ACS }\end{array}$ & $\begin{array}{r}\text { GSS } \\
2004- \\
2016 \\
\end{array}$ \\
\hline $\mathrm{V}$ & Actors & 2700 & 2700 & & 2 \\
\hline $\mathrm{V}$ & Agricultural And Food Science Technicians & 1900 & 1900 & & 8 \\
\hline $\mathrm{V}$ & Announcers & 2800 & 2800 & & 7 \\
\hline V & Artists And Related Workers & 2600 & 2600 & & 14 \\
\hline \multirow[t]{2}{*}{ V } & Athletes, Coaches, Umpires, And Related & & & & \\
\hline & Workers & 2720 & 2720 & & 19 \\
\hline $\mathrm{V}$ & Avionics Technicians & 7030 & 7030 & & 2 \\
\hline $\mathrm{V}$ & Bailiffs, Correctional Officers, And Jailers & 3800 & 3800 & & 49 \\
\hline $\mathrm{V}$ & Biological Technicians & 1910 & 1910 & & 6 \\
\hline \multirow[t]{2}{*}{$\mathrm{V}$} & $\begin{array}{l}\text { Broadcast And Sound Engineering } \\
\text { Technicians And Radio Operators, And Media } \\
\text { And Communication Equipment Workers, All }\end{array}$ & & & & \\
\hline & Other & 2900 & 2900 & & 11 \\
\hline V & $\begin{array}{l}\text { Buyers And Purchasing Agents, Farm } \\
\text { Products }\end{array}$ & 510 & 510 & & 1 \\
\hline V & Chefs And Head Cooks & 4000 & 4000 & & 38 \\
\hline V & Chemical Technicians & 1920 & 1920 & & 9 \\
\hline V & $\begin{array}{l}\text { Clinical Laboratory Technologists And } \\
\text { Technicians }\end{array}$ & 3300 & 3300 & & 48 \\
\hline V & $\begin{array}{l}\text { Computer Control Programmers And } \\
\text { Operators }\end{array}$ & 7900 & 7900 & & 1 \\
\hline $\mathrm{V}$ & Construction And Building Inspectors & 6660 & 6660 & & 7 \\
\hline $\mathrm{V}$ & Construction Managers & 220 & 220 & & 51 \\
\hline V & Cost Estimators & 600 & 600 & & 5 \\
\hline $\mathrm{V}$ & Dancers And Choreographers & 2740 & 2740 & & 1 \\
\hline $\mathrm{V}$ & Dental Hygienists & 3310 & 3310 & & 9 \\
\hline V & Designers & 2630 & 2630 & & 66 \\
\hline $\mathrm{V}$ & Detectives And Criminal Investigators & 3820 & 3820 & & 16 \\
\hline V & $\begin{array}{l}\text { Diagnostic Related Technologists And } \\
\text { Technicians }\end{array}$ & 3320 & 3320 & & 40 \\
\hline V & Drafters & 1540 & 1540 & & 19 \\
\hline V & $\begin{array}{l}\text { Electric Motor, Power Tool, And Related } \\
\text { Repairers }\end{array}$ & 7040 & 7040 & & 5 \\
\hline V & $\begin{array}{l}\text { Electrical And Electronics Repairers, } \\
\text { Transportation Equipment, And Industrial } \\
\text { And Utility }\end{array}$ & 7100 & 7100 & & 1 \\
\hline V & $\begin{array}{l}\text { Electronic Equipment Installers And } \\
\text { Repairers, Motor Vehicles }\end{array}$ & 7110 & 7110 & & 4 \\
\hline V & $\begin{array}{l}\text { Electronic Home Entertainment Equipment } \\
\text { Installers And Repairers }\end{array}$ & 7120 & 7120 & & 2 \\
\hline $\mathrm{V}$ & Elevator Installers And Repairers & 6700 & 6700 & & 1 \\
\hline V & $\begin{array}{l}\text { Emergency Medical Technicians And } \\
\text { Paramedics }\end{array}$ & 3400 & 3400 & & 22 \\
\hline V & Engineering Technicians, Except Drafters & 1550 & 1550 & & 55 \\
\hline $\mathrm{V}$ & $\begin{array}{l}\text { Entertainers And Performers, Sports And } \\
\text { Related Workers, All Other }\end{array}$ & 2760 & 2760 & & 1 \\
\hline V & Fire Inspectors & 3750 & 3750 & & 5 \\
\hline
\end{tabular}




\begin{tabular}{|c|c|c|c|c|c|}
\hline Class & Occupational Title (ACS 2012) & $\begin{array}{l}\text { ACS } \\
2012 \\
\end{array}$ & $\begin{array}{r}\text { GSS } \\
\text { occ10 }\end{array}$ & $\begin{array}{l}\text { Recoded } \\
\text { to ACS }\end{array}$ & $\begin{array}{r}\text { GSS } \\
2004- \\
2016 \\
\end{array}$ \\
\hline $\mathrm{V}$ & Firefighters & 3740 & 3740 & & 36 \\
\hline $\mathrm{V}$ & $\begin{array}{l}\text { First-Line Supervisors Of Construction Trades } \\
\text { And Extraction Workers }\end{array}$ & & 6200 & & 63 \\
\hline $\mathrm{V}$ & First-Line Supervisors Of Correctional Officers & $\begin{array}{l}6200 \\
3700\end{array}$ & $\begin{array}{l}6200 \\
3700\end{array}$ & & 11 \\
\hline $\mathrm{V}$ & $\begin{array}{l}\text { First-Line Supervisors Of Landscaping, Lawn } \\
\text { Service, And Groundskeeping Workers }\end{array}$ & 4210 & 4210 & & 13 \\
\hline $\mathrm{V}$ & $\begin{array}{l}\text { First-Line Supervisors Of Mechanics, } \\
\text { Installers, And Repairers }\end{array}$ & 7000 & 7000 & & 40 \\
\hline $\mathrm{V}$ & $\begin{array}{l}\text { First-Line Supervisors Of Non-Retail Sales } \\
\text { Workers }\end{array}$ & 4710 & 4710 & & 62 \\
\hline $\mathrm{V}$ & $\begin{array}{l}\text { First-Line Supervisors Of Production And } \\
\text { Operating Workers }\end{array}$ & 7700 & 7700 & & 102 \\
\hline $\mathrm{V}$ & $\begin{array}{l}\text { First-Line Supervisors Of Protective Service } \\
\text { Workers, All Other }\end{array}$ & 3730 & 3730 & & 14 \\
\hline $\mathrm{V}$ & $\begin{array}{l}\text { First-Line Supervisors Of Fire Fighting And } \\
\text { Prevention Workers }\end{array}$ & 3720 & 3720 & & 3 \\
\hline $\mathrm{V}$ & $\begin{array}{l}\text { First-Line Supervisors Of Police And } \\
\text { Detectives }\end{array}$ & 3710 & 3710 & & 10 \\
\hline $\mathrm{V}$ & Gaming Managers & 330 & 330 & & 3 \\
\hline $\mathrm{V}$ & $\begin{array}{l}\text { Geological And Petroleum Technicians, And } \\
\text { Nuclear Technicians }\end{array}$ & 1930 & 1930 & & 4 \\
\hline $\mathrm{V}$ & $\begin{array}{l}\text { Health Practitioner Support Technologists } \\
\text { And Technicians }\end{array}$ & 3420 & 3420 & & 78 \\
\hline $\mathrm{V}$ & $\begin{array}{l}\text { Miscellaneous Health Technologists And } \\
\text { Technicians }\end{array}$ & 3535 & 3535 & & 12 \\
\hline $\mathrm{V}$ & Miscellaneous Law Enforcement Workers & 3840 & 3840 & & 1 \\
\hline $\mathrm{V}$ & $\begin{array}{l}\text { Miscellaneous Life, Physical, And Social } \\
\text { Science Technicians, Including Social Science } \\
\text { Research Assistants }\end{array}$ & 1965 & 1965 & & 26 \\
\hline $\mathrm{V}$ & $\begin{array}{l}\text { Miscellaneous Life, Physical, And Social } \\
\text { Science Technicians, Including Social Science }\end{array}$ & & & & \\
\hline & Research Assistants & 1965 & 1950 & $\mathrm{x}$ & 2 \\
\hline $\mathrm{V}$ & $\begin{array}{l}\text { Miscellaneous Media And Communication } \\
\text { Workers }\end{array}$ & 2860 & 2860 & & 8 \\
\hline $\mathrm{V}$ & Musicians, Singers, And Related Workers & 2750 & 2750 & & 10 \\
\hline $\mathrm{V}$ & Opticians, Dispensing & 3520 & 3520 & & 10 \\
\hline $\mathrm{V}$ & Photographers & 2910 & 2910 & & 10 \\
\hline V & Police Officers & 3850 & 3850 & & 81 \\
\hline $\mathrm{V}$ & Private Detectives And Investigators & 3910 & 3910 & & 11 \\
\hline $\mathrm{V}$ & $\begin{array}{l}\text { Probation Officers And Correctional } \\
\text { Treatment Specialists }\end{array}$ & 2015 & 2015 & & 7 \\
\hline $\mathrm{V}$ & $\begin{array}{l}\text { Property, Real Estate, And Community } \\
\text { Association Managers }\end{array}$ & 410 & 410 & & 47 \\
\hline $\mathrm{V}$ & Radio And Telecommunications Equipment & & & & \\
\hline & Installers And Repairers & 7020 & 7020 & & 25 \\
\hline $\mathrm{V}$ & Railroad Conductors And Yardmasters & 9240 & 9240 & & 7 \\
\hline $\mathrm{V}$ & Ship And Boat Captains And Operators & 9310 & 9310 & & 2 \\
\hline
\end{tabular}




\begin{tabular}{|c|c|c|c|c|c|}
\hline Class & Occupational Title (ACS 2012) & $\begin{array}{l}\text { ACS } \\
2012\end{array}$ & $\begin{array}{r}\text { GSS } \\
\text { occ10 }\end{array}$ & $\begin{array}{l}\text { Recoded } \\
\text { to ACS }\end{array}$ & $\begin{array}{r}\text { GSS } \\
2004- \\
2016\end{array}$ \\
\hline \multirow[t]{2}{*}{$\mathrm{V}$} & Supervisors Of Transportation And Material & & & & \\
\hline & Moving Workers & 9000 & 9000 & & 33 \\
\hline $\mathrm{V}$ & Surveying And Mapping Technicians & 1560 & 1560 & & 11 \\
\hline \multirow[t]{2}{*}{$\mathrm{V}$} & Telecommunications Line Installers And & & & & \\
\hline & Repairers & 7420 & 7420 & & 18 \\
\hline \multirow[t]{2}{*}{$\mathrm{V}$} & Television, Video, And Motion Picture Camera & & & & \\
\hline & Operators And Editors & 2920 & 2920 & & 3 \\
\hline $\mathrm{V}$ & Transportation Inspectors & 9410 & 9410 & & 7 \\
\hline \multirow[t]{2}{*}{$\mathrm{V}$} & Transportation, Storage, And Distribution & & & & \\
\hline & Managers & 160 & 160 & & 26 \\
\hline VI & Aircraft Mechanics And Service Technicians & 7140 & 7140 & & 21 \\
\hline \multirow[t]{2}{*}{ VI } & Automotive Service Technicians And & & & & \\
\hline & Mechanics & 7200 & 7200 & & 79 \\
\hline VI & Boilermakers & 6210 & 6210 & & 3 \\
\hline \multirow[t]{2}{*}{ VI } & Brickmasons, Blockmasons, Stonemasons, And & & & & \\
\hline & Reinforcing Iron And Rebar Workers & 6220 & 6220 & & 18 \\
\hline \multirow[t]{2}{*}{ VI } & Bus And Truck Mechanics And Diesel Engine & & & & \\
\hline & Specialists & 7210 & 7210 & & 35 \\
\hline VI & Cabinetmakers And Bench Carpenters & 8500 & 8500 & & 5 \\
\hline VI & Carpenters & 6230 & 6230 & & 112 \\
\hline \multirow[t]{2}{*}{ VI } & Carpet, Floor, And Tile Installers And & & & & \\
\hline & Finishers & 6240 & 6240 & & 16 \\
\hline \multirow[t]{2}{*}{ VI } & Computer, Automated Teller, And Office & & & & \\
\hline & Machine Repairers & 7010 & 7010 & & 22 \\
\hline \multirow[t]{2}{*}{ VI } & $\begin{array}{l}\text { Construction Equipment Operators, Except } \\
\text { Paving, Surfacing, And Tamping Equipment }\end{array}$ & & & & \\
\hline & Operators & 6320 & 6320 & & 46 \\
\hline VI & Control And Valve Installers And Repairers & 7300 & 7300 & & 2 \\
\hline VI & Crane And Tower Operators & 9510 & 9510 & & 12 \\
\hline \multirow[t]{2}{*}{ VI } & Drywall Installers, Ceiling Tile Installers, And & & & & \\
\hline & Tapers & 6330 & 6330 & & 13 \\
\hline VI & Electrical Power-Line Installers And Repairers & 7410 & 7410 & & 12 \\
\hline VI & Electricians & 6355 & 6355 & & 67 \\
\hline \multirow[t]{2}{*}{ VI } & Explosives Workers, Ordnance Handling & & & & \\
\hline & Experts, And Blasters & 6830 & 6830 & & 4 \\
\hline VI & Forest And Conservation Workers & 6120 & 6120 & & 2 \\
\hline VI & Hazardous Materials Removal Workers & 6720 & 6720 & & 4 \\
\hline \multirow[t]{2}{*}{ VI } & Heating, Air Conditioning, And Refrigeration & & & & \\
\hline & Mechanics And Installers & 7315 & 7315 & & 35 \\
\hline \multirow[t]{2}{*}{ VI } & Heavy Vehicle And Mobile Equipment Service & & & & \\
\hline & Technicians And Mechanics & 7220 & 7220 & & 22 \\
\hline VI & Home Appliance Repairers & 7320 & 7320 & & 7 \\
\hline \multirow[t]{2}{*}{ VI } & Industrial And Refractory Machinery & & & & \\
\hline & Mechanics & 7330 & 7330 & & 29 \\
\hline VI & Insulation Workers & 6400 & 6400 & & 7 \\
\hline \multirow[t]{2}{*}{ VI } & Jewelers And Precious Stone And Metal & & & & \\
\hline & Workers & 8750 & 8750 & & 5 \\
\hline VI & Locksmiths And Safe Repairers & 7540 & 7540 & & 3 \\
\hline
\end{tabular}




\begin{tabular}{|c|c|c|c|c|c|}
\hline Class & Occupational Title (ACS 2012) & $\begin{array}{l}\text { ACS } \\
2012 \\
\end{array}$ & $\begin{array}{r}\text { GSS } \\
\text { occ10 }\end{array}$ & $\begin{array}{l}\text { Recoded } \\
\text { to ACS }\end{array}$ & $\begin{array}{r}\text { GSS } \\
2004- \\
2016 \\
\end{array}$ \\
\hline VI & Locomotive Engineers And Operators & 9200 & 9200 & & 7 \\
\hline VI & Machinists & 8030 & 8030 & & 48 \\
\hline VI & Maintenance And Repair Workers, General & 7340 & 7340 & & 26 \\
\hline VI & Maintenance Workers, Machinery & 7350 & 7350 & & 5 \\
\hline VI & $\begin{array}{l}\text { Medical, Dental, And Ophthalmic Laboratory } \\
\text { Technicians }\end{array}$ & 8760 & 8760 & & 9 \\
\hline VI & Millwrights & 7360 & 7360 & & 18 \\
\hline VI & $\begin{array}{l}\text { Miscellaneous Installation, Maintenance, And } \\
\text { Repair Workers, Including Wind Turbine } \\
\text { Service Technicians }\end{array}$ & 7630 & 7630 & & 21 \\
\hline VI & Miscellaneous Plant And System Operators & 8630 & 8630 & & 8 \\
\hline VI & $\begin{array}{l}\text { Miscellaneous Woodworkers, Including } \\
\text { Model Makers And Patternmakers }\end{array}$ & 8550 & 8550 & & 1 \\
\hline VI & $\begin{array}{l}\text { Model Makers, Patternmakers, And Molding } \\
\text { Machine Setters, Metal And Plastic }\end{array}$ & 8100 & 8100 & & 13 \\
\hline VI & Model Makers, Patternmakers, And Molding & & & & \\
\hline VI & $\begin{array}{l}\text { Machine Setters, Metal And Plastic } \\
\text { Molders, Shapers, And Casters, Except Metal }\end{array}$ & 8100 & 8060 & $\mathrm{x}$ & 1 \\
\hline & And Plastic & 8920 & 8920 & & 6 \\
\hline VI & Painters And Paperhangers & 6420 & 6420 & & 30 \\
\hline VI & $\begin{array}{l}\text { Pipelayers, Plumbers, Pipefitters, And } \\
\text { Steamfitters }\end{array}$ & 6440 & 6440 & & 54 \\
\hline VI & $\begin{array}{l}\text { Power Plant Operators, Distributors, And } \\
\text { Dispatchers }\end{array}$ & 8600 & 8600 & & 2 \\
\hline VI & $\begin{array}{l}\text { Precision Instrument And Equipment } \\
\text { Repairers }\end{array}$ & 7430 & 7430 & & 9 \\
\hline VI & Prepress Technicians And Workers & 8250 & 8250 & & 11 \\
\hline VI & Printing Press Operators & 8255 & 8255 & & 23 \\
\hline VI & $\begin{array}{l}\text { Sailors And Marine Oilers, And Ship } \\
\text { Engineers }\end{array}$ & 9300 & 9300 & & 2 \\
\hline VI & Security And Fire Alarm Systems Installers & 7130 & 7130 & & 3 \\
\hline VI & Sheet Metal Workers & 6520 & 6520 & & 15 \\
\hline VI & Small Engine Mechanics & 7240 & 7240 & & 9 \\
\hline VI & Stationary Engineers And Boiler Operators & 8610 & 8610 & & 17 \\
\hline VI & Structural Iron And Steel Workers & 6530 & 6530 & & 12 \\
\hline VI & Structural Metal Fabricators And Fitters & 7740 & 7740 & & 7 \\
\hline VI & $\begin{array}{l}\text { Subway, Streetcar, And Other Rail } \\
\text { Transportation Workers }\end{array}$ & 9260 & 9260 & & 2 \\
\hline VI & $\begin{array}{l}\text { Subway, Streetcar, And Other Rail } \\
\text { Transportation Workers }\end{array}$ & 9260 & 9230 & $\mathrm{x}$ & 1 \\
\hline VI & Tailors, Dressmakers, And Sewers & 8350 & 8350 & & 11 \\
\hline VI & Tool And Die Makers & 8130 & 8130 & & 14 \\
\hline VI & $\begin{array}{l}\text { Water And Wastewater Treatment Plant And } \\
\text { System Operators }\end{array}$ & 8620 & 8620 & & 9 \\
\hline VIIa & $\begin{array}{l}\text { Adhesive Bonding Machine Operators And } \\
\text { Tenders }\end{array}$ & 8850 & 8850 & & 2 \\
\hline
\end{tabular}




\begin{tabular}{|c|c|c|c|c|c|}
\hline Class & Occupational Title (ACS 2012) & $\begin{array}{l}\text { ACS } \\
2012\end{array}$ & $\begin{array}{r}\text { GSS } \\
\text { occ10 }\end{array}$ & $\begin{array}{l}\text { Recoded } \\
\text { to ACS }\end{array}$ & $\begin{array}{r}\text { GSS } \\
2004- \\
2016 \\
\end{array}$ \\
\hline VIIa & $\begin{array}{l}\text { Aircraft Structure, Surfaces, Rigging, And } \\
\text { Systems Assemblers }\end{array}$ & 7710 & 7710 & & 6 \\
\hline VIIa & Automotive Body And Related Repairers & 7150 & 7150 & & 22 \\
\hline VIIa & Automotive Glass Installers And Repairers & 7160 & 7160 & & 2 \\
\hline VIIa & Bakers & 7800 & 7800 & & 23 \\
\hline VIIa & $\begin{array}{l}\text { Butchers And Other Meat, Poultry, And Fish } \\
\text { Processing Workers }\end{array}$ & 7810 & 7810 & & 33 \\
\hline VIIa & $\begin{array}{l}\text { Cement Masons, Concrete Finishers, And } \\
\text { Terrazzo Workers }\end{array}$ & 6250 & 6250 & & 9 \\
\hline VIIa & $\begin{array}{l}\text { Chemical Processing Machine Setters, } \\
\text { Operators, And Tenders }\end{array}$ & 8640 & 8640 & & 12 \\
\hline VIIa & Cleaners Of Vehicles And Equipment & 9610 & 9610 & & 17 \\
\hline VIIa & Construction Laborers & 6260 & 6260 & & 150 \\
\hline VIIa & $\begin{array}{l}\text { Conveyor Operators And Tenders, And Hoist } \\
\text { And Winch Operators }\end{array}$ & 9560 & 9500 & $\mathrm{x}$ & 1 \\
\hline VIIa & Cooks & 4020 & 4020 & & 234 \\
\hline VIIa & Crossing Guards & 3940 & 3940 & & 8 \\
\hline VIIa & $\begin{array}{l}\text { Crushing, Grinding, Polishing, Mixing, And } \\
\text { Blending Workers }\end{array}$ & 8650 & 8650 & & 11 \\
\hline VIIa & Cutting Workers & 8710 & 8710 & & 12 \\
\hline VIIa & $\begin{array}{l}\text { Derrick, Rotary Drill, And Service Unit } \\
\text { Operators, And Roustabouts, Oil, Gas, And } \\
\text { Mining }\end{array}$ & 6800 & 6800 & & 3 \\
\hline VIIa & Dishwashers & 4140 & 4140 & & 35 \\
\hline VIIa & $\begin{array}{l}\text { Dredge, Excavating, And Loading Machine } \\
\text { Operators }\end{array}$ & 9520 & 9520 & & 9 \\
\hline VIIa & Driver/Sales Workers And Truck Drivers & 9130 & 9130 & & 326 \\
\hline VIIa & Earth Drillers, Except Oil And Gas & 6820 & 6820 & & 2 \\
\hline VIIa & $\begin{array}{l}\text { Electrical, Electronics, And Electromechanical } \\
\text { Assemblers }\end{array}$ & 7720 & 7720 & & 30 \\
\hline VIIa & Engine And Other Machine Assemblers & 7730 & 7730 & & 7 \\
\hline VIIa & $\begin{array}{l}\text { Extruding And Drawing Machine Setters, } \\
\text { Operators, And Tenders, Metal And Plastic }\end{array}$ & 7920 & 7920 & & 3 \\
\hline VIIa & $\begin{array}{l}\text { Extruding, Forming, Pressing, And } \\
\text { Compacting Machine Setters, Operators, And }\end{array}$ & & & & \\
\hline & Tenders & 8720 & 8720 & & 10 \\
\hline VIIa & Fence Erectors & 6710 & 6710 & & 1 \\
\hline VIIa & $\begin{array}{l}\text { Food And Tobacco Roasting, Baking, And } \\
\text { Drying Machine Operators And Tenders }\end{array}$ & 7830 & 7830 & & 6 \\
\hline VIIa & Food Batchmakers & 7840 & 7840 & & 12 \\
\hline VIIa & $\begin{array}{l}\text { Food Cooking Machine Operators And } \\
\text { Tenders }\end{array}$ & 7850 & 7850 & & 1 \\
\hline VIIa & Food Preparation Workers & 4030 & 4030 & & 68 \\
\hline VIIa & Food Processing Workers, All Other & 7855 & 7855 & & 15 \\
\hline VIIa & $\begin{array}{l}\text { Forging Machine Setters, Operators, And } \\
\text { Tenders, Metal And Plastic }\end{array}$ & 7930 & 7930 & & 2 \\
\hline
\end{tabular}




\begin{tabular}{|c|c|c|c|c|c|}
\hline Class & Occupational Title (ACS 2012) & $\begin{array}{l}\text { ACS } \\
2012\end{array}$ & $\begin{array}{r}\text { GSS } \\
\text { occ10 }\end{array}$ & $\begin{array}{l}\text { Recoded } \\
\text { to ACS }\end{array}$ & $\begin{array}{r}\text { GSS } \\
2004- \\
2016\end{array}$ \\
\hline \multirow[t]{2}{*}{ VIIa } & Furnace, Kiln, Oven, Drier, And Kettle & & & & \\
\hline & Operators And Tenders & 8730 & 8730 & & 1 \\
\hline VIIa & Furniture Finishers & 8510 & 8510 & & 5 \\
\hline VIIa & Glaziers & 6360 & 6360 & & 8 \\
\hline VIIa & Grounds Maintenance Workers & 4250 & 4250 & & 86 \\
\hline VIIa & Helpers, Construction Trades & 6600 & 6600 & & 9 \\
\hline \multirow[t]{2}{*}{ VIIa } & Helpers-Installation, Maintenance, And Repair & & & & \\
\hline & Workers & 7610 & 7610 & & 6 \\
\hline VIIa & Helpers-Production Workers & 8950 & 8950 & & 7 \\
\hline VIIa & Highway Maintenance Workers & 6730 & 6730 & & 11 \\
\hline VIIa & Industrial Truck And Tractor Operators & 9600 & 9600 & & 62 \\
\hline VIIa & $\begin{array}{l}\text { Inspectors, Testers, Sorters, Samplers, And } \\
\text { Weighers }\end{array}$ & 8740 & 8740 & & 98 \\
\hline VIIa & Janitors And Building Cleaners & 4220 & 4220 & & 288 \\
\hline VIIa & $\begin{array}{l}\text { Laborers And Freight, Stock, And Material } \\
\text { Movers, Hand }\end{array}$ & 9620 & 9620 & & 172 \\
\hline VIIa & Laundry And Dry-Cleaning Workers & 8300 & 8300 & & 21 \\
\hline VIIa & Logging Workers & 6130 & 6130 & & 16 \\
\hline VIIa & Machine Feeders And Offbearers & 9630 & 9630 & & 11 \\
\hline VIIa & $\begin{array}{l}\text { Machine Tool Cutting Setters, Operators, And } \\
\text { Tenders, Metal And Plastic }\end{array}$ & 7950 & 7950 & & 21 \\
\hline VIIa & $\begin{array}{l}\text { Machine Tool Cutting Setters, Operators, And } \\
\text { Tenders, Metal And Plastic }\end{array}$ & 7950 & 8000 & $\mathrm{x}$ & 9 \\
\hline VIIa & $\begin{array}{l}\text { Machine Tool Cutting Setters, Operators, And } \\
\text { Tenders, Metal And Plastic }\end{array}$ & 7950 & 7960 & $\mathrm{x}$ & 3 \\
\hline VIIa & $\begin{array}{l}\text { Machine Tool Cutting Setters, Operators, And } \\
\text { Tenders, Metal And Plastic }\end{array}$ & 7950 & 8010 & $\mathrm{x}$ & 4 \\
\hline VIIa & Maids And Housekeeping Cleaners & 4230 & 4230 & & 178 \\
\hline VIIa & $\begin{array}{l}\text { Mail Clerks And Mail Machine Operators, } \\
\text { Except Postal Service }\end{array}$ & 5850 & 5850 & & 10 \\
\hline VIIa & $\begin{array}{l}\text { Metal Furnace Operators, Tenders, Pourers, } \\
\text { And Casters }\end{array}$ & 8040 & 8040 & & 3 \\
\hline VIIa & Mining Machine Operators & 6840 & 6840 & & 9 \\
\hline VIIa & Miscellaneous Assemblers And Fabricators & 7750 & 7750 & & 103 \\
\hline VIIa & $\begin{array}{l}\text { Miscellaneous Construction Workers, } \\
\text { Including Solar Photovoltaic Installers, Septic }\end{array}$ & & & & \\
\hline \multirow[t]{2}{*}{ VIIa } & $\begin{array}{l}\text { Tank Servicers And Sewer Pipe Cleaners } \\
\text { Miscellaneous Construction Workers, } \\
\text { Including Solar Photovoltaic Installers, Septic }\end{array}$ & 6765 & 6765 & & 4 \\
\hline & $\begin{array}{l}\text { Tank Servicers And Sewer Pipe Cleaners } \\
\text { Miscellaneous Extraction Workers, Including }\end{array}$ & 6765 & 6750 & $\mathrm{x}$ & 1 \\
\hline VIIa & Roof Bolters And Helpers & 6940 & 6940 & & 2 \\
\hline \multirow[b]{2}{*}{ VIIa } & $\begin{array}{l}\text { Miscellaneous Material Moving Workers, } \\
\text { Including Mine Shuttle Car Operators, And } \\
\text { Tank Car, Truck, And Ship Loaders }\end{array}$ & 9750 & 9750 & & 13 \\
\hline & $\begin{array}{l}\text { Miscellaneous Material Moving Workers, } \\
\text { Including Mine Shuttle Car Operators, And } \\
\text { Tank Car, Truck, And Ship Loaders }\end{array}$ & 9750 & 9730 & $\mathrm{x}$ & 2 \\
\hline
\end{tabular}




\begin{tabular}{|c|c|c|c|c|c|}
\hline Class & Occupational Title (ACS 2012) & $\begin{array}{l}\text { ACS } \\
2012\end{array}$ & $\begin{array}{r}\text { GSS } \\
\text { occ10 }\end{array}$ & $\begin{array}{l}\text { Recoded } \\
\text { to ACS }\end{array}$ & $\begin{array}{r}\text { GSS } \\
2004- \\
2016\end{array}$ \\
\hline VIIa & $\begin{array}{l}\text { Miscellaneous Metal Workers And Plastic } \\
\text { Workers, Including Multiple Machine Tool }\end{array}$ & 8220 & 8220 & & 41 \\
\hline VIIa & $\begin{array}{l}\text { Miscellaneous Metal Workers And Plastic } \\
\text { Workers, Including Multiple Machine Tool }\end{array}$ & $8 \angle \angle U$ & $8 \angle \angle O$ & & 41 \\
\hline VIIa & $\begin{array}{l}\text { Setters } \\
\text { Miscellaneous Metal Workers And Plastic } \\
\text { Workers, Including Multiple Machine Tool }\end{array}$ & 8220 & 8020 & $\mathrm{x}$ & 1 \\
\hline VIIa & $\begin{array}{l}\text { Setters } \\
\text { Miscellaneous Metal Workers And Plastic } \\
\text { Workers, Including Multiple Machine Tool }\end{array}$ & 8220 & 8210 & $\mathrm{x}$ & 2 \\
\hline VIIa & $\begin{array}{l}\text { Setters } \\
\text { Miscellaneous Metal Workers And Plastic } \\
\text { Workers, Including Multiple Machine Tool } \\
\text { Setters }\end{array}$ & 8220 & 8120 & $\mathrm{x}$ & 2 \\
\hline VIIa & $\begin{array}{l}\text { Miscellaneous Production Workers, Including } \\
\text { Semiconductor Processors }\end{array}$ & 8965 & 8965 & & 183 \\
\hline VIIa & $\begin{array}{l}\text { Miscellaneous Production Workers, Including } \\
\text { Semiconductor Processors } \\
\text { Miscellaneous Textile, Apparel, And }\end{array}$ & 8965 & 8860 & $\mathrm{x}$ & 1 \\
\hline VIIa & $\begin{array}{l}\text { Furnishings Workers, Except Upholsterers } \\
\text { Miscellaneous Vehicle And Mobile Equipment }\end{array}$ & 8460 & 8460 & & 8 \\
\hline & Mechanics, Installers, And Repairers & 7260 & 7260 & & 11 \\
\hline VIIa & Motor Vehicle Operators, All Other & 9150 & 9150 & & 1 \\
\hline VIIa & $\begin{array}{l}\text { Packaging And Filling Machine Operators } \\
\text { And Tenders }\end{array}$ & 8800 & 8800 & & 30 \\
\hline VIIa & Packers And Packagers, Hand & 9640 & 9640 & & 54 \\
\hline VIIa & Painting Workers & 8810 & 8810 & & 18 \\
\hline VIIa & $\begin{array}{l}\text { Paper Goods Machine Setters, Operators, And } \\
\text { Tenders }\end{array}$ & 8930 & 8930 & & 7 \\
\hline VIIa & $\begin{array}{l}\text { Paving, Surfacing, And Tamping Equipment } \\
\text { Operators }\end{array}$ & 6300 & 6300 & & 1 \\
\hline VIIa & $\begin{array}{l}\text { Photographic Process Workers And Processing } \\
\text { Machine Operators }\end{array}$ & 8830 & 8830 & & 11 \\
\hline VIIa & Plasterers And Stucco Masons & 6460 & 6460 & & 3 \\
\hline VIIa & Postal Service Mail Carriers & 5550 & 5550 & & 40 \\
\hline VIIa & $\begin{array}{l}\text { Postal Service Mail Sorters, Processors, And } \\
\text { Processing Machine Operators }\end{array}$ & 5560 & 5560 & & 12 \\
\hline VIIa & $\begin{array}{l}\text { Pressers, Textile, Garment, And Related } \\
\text { Materials }\end{array}$ & 8310 & 8310 & & 13 \\
\hline VIIa & Print Binding And Finishing Workers & 8256 & 8256 & & 7 \\
\hline VIIa & Pumping Station Operators & 9650 & 9650 & & 5 \\
\hline VIIa & Rail-Track Laying And Maintenance & & & & \\
\hline & Equipment Operators & 6740 & 6740 & & 3 \\
\hline VIIa & Refuse And Recyclable Material Collectors & 9720 & 9720 & & 17 \\
\hline VIIa & $\begin{array}{l}\text { Rolling Machine Setters, Operators, And } \\
\text { Tenders, Metal And Plastic }\end{array}$ & 7940 & 7940 & & 1 \\
\hline VIIa & Roofers & 6515 & 6515 & & 24 \\
\hline
\end{tabular}




\begin{tabular}{|c|c|c|c|c|c|}
\hline Class & Occupational Title (ACS 2012) & $\begin{array}{l}\text { ACS } \\
2012\end{array}$ & $\begin{array}{r}\text { GSS } \\
\text { occ10 }\end{array}$ & $\begin{array}{l}\text { Recoded } \\
\text { to ACS }\end{array}$ & $\begin{array}{r}\text { GSS } \\
2004- \\
2016\end{array}$ \\
\hline VIIa & $\begin{array}{l}\text { Sawing Machine Setters, Operators, And } \\
\text { Tenders, Wood }\end{array}$ & 8530 & 8530 & & 11 \\
\hline VIIa & Sewing Machine Operators & 8320 & 8320 & & 77 \\
\hline VIIa & Shipping, Receiving, And Traffic Clerks & 5610 & 5610 & & 52 \\
\hline VIIa & Shoe And Leather Workers & 8330 & 8330 & & 2 \\
\hline VIIa & Shoe And Leather Workers & 8330 & 8340 & $\mathrm{x}$ & 3 \\
\hline VIIa & Stock Clerks And Order Fillers & 5620 & 5620 & & 125 \\
\hline VIIa & Taxi Drivers And Chauffeurs & 9140 & 9140 & & 23 \\
\hline VIIa & $\begin{array}{l}\text { Textile Bleaching And Dyeing, And Cutting } \\
\text { Machine Setters, Operators, And Tenders } \\
\text { Textile Bleaching And Dyeing, And Cutting }\end{array}$ & 8400 & 8400 & & 1 \\
\hline VIIa & $\begin{array}{l}\text { Machine Setters, Operators, And Tenders } \\
\text { Textile Knitting And Weaving Machine }\end{array}$ & 8400 & 8360 & $x$ & 2 \\
\hline & Setters, Operators, And Tenders & 8410 & 8410 & & 3 \\
\hline VIIa & $\begin{array}{l}\text { Textile Winding, Twisting, And Drawing Out } \\
\text { Machine Setters, Operators, And Tenders }\end{array}$ & 8420 & 8420 & & 12 \\
\hline VIIa & Tire Builders & 8940 & 8940 & & 3 \\
\hline VIIa & Upholsterers & 8450 & 8450 & & 6 \\
\hline VIIa & Welding, Soldering, And Brazing Workers & 8140 & 8140 & & 74 \\
\hline VIIa & $\begin{array}{l}\text { Woodworking Machine Setters, Operators, } \\
\text { And Tenders, Except Sawing }\end{array}$ & 8540 & 8540 & & 8 \\
\hline VIIb & Agricultural Inspectors & 6010 & 6010 & & 3 \\
\hline VIIb & Animal Trainers & 4340 & 4340 & & 5 \\
\hline VIIb & $\begin{array}{l}\text { First-Line Supervisors Of Farming, Fishing, } \\
\text { And Forestry Workers }\end{array}$ & 6005 & 6005 & & 9 \\
\hline VIIb & Fishing And Hunting Workers & 6100 & 6100 & & 2 \\
\hline VIIb & Graders And Sorters, Agricultural Products & 6040 & 6040 & & 9 \\
\hline VIIb & $\begin{array}{l}\text { Miscellaneous Agricultural Workers, } \\
\text { Including Animal Breeders }\end{array}$ & 6050 & 6050 & & 97 \\
\hline Mil & First-Line Enlisted Military Supervisors & 9810 & 9810 & & 46 \\
\hline Mil & $\begin{array}{l}\text { Military Enlisted Tactical Operations And } \\
\text { Air/Weapons Specialists And Crew Members }\end{array}$ & 9820 & 9820 & & 18 \\
\hline Mil & $\begin{array}{l}\text { Military Officer Special And Tactical } \\
\text { Operations Leaders }\end{array}$ & 9800 & 9800 & & 48 \\
\hline Mil & Military, Rank Not Specified & 9830 & 9830 & & 60 \\
\hline
\end{tabular}

